\title{
Reversible Stereoisomerization of 1,3- Diphosphetane Frameworks Revealed by a Single-Electron Redox Approach
}

Xiaodan Chen, ${ }^{[a]} \ddagger$ Chenyang Hu, ${ }^{[b]} \ddagger$ Xu Zhang, ${ }^{[a]}$ Shihua Liu, ${ }^{[c]}$ Yanbo Mei, ${ }^{[b]}$ Guping $H u,{ }^{[c]}$ Liu Leo Liu, ${ }^{[b]}$ Zhongshu Li, ${ }^{*[a, c]}$ Cheng-Yong Su ${ }^{*[c]}$

[a] Guangdong Provincial Key Laboratory of Functional Supramolecular Coordination Materials and Applications, College of Chemistry and Materials Science, Jinan University, Guangzhou 510632 (China)

[b] Shenzhen Grubbs Institute and Department of Chemistry, Southern University of Science and Technology, Shenzhen 518055 (China)

[c] Lehn Institute of Functional Materials (LIFM), School of Chemistry, Sun Yat-Sen University, Guangzhou 510275 (China) 


\title{
Supporting Information
}

\author{
S1: Synthetic Details \\ S2: X-Ray Diffraction Studies \\ S3: CV and EPR analysis \\ S4: Theoretical Details \\ S5: References
}

\section{S1: Synthetic Details}

General: All manipulations were performed under an inert atmosphere of dry nitrogen, using standard Schlenk techniques. Dry, oxygen-free solvents were employed unless otherwise mentioned. The compound $\mathbf{1}$ were prepared following our recent procedure, ${ }^{[1]}$ while all other starting materials were purchased from commercial sources. NMR spectra were recorded on Bruker Avance $400 \mathrm{MHz}$ spectrometers $\left({ }^{1} \mathrm{H}, 400.1 \mathrm{MHz} ;{ }^{13} \mathrm{C}\right.$, 100.5 MHz; $\left.{ }^{31} \mathrm{P}, 161.9 \mathrm{MHz}\right)$. All spectra were obtained in the solvent indicated at $25^{\circ} \mathrm{C}$. The chemical shifts $(\delta)$ were measured according to IUPAC and expressed in ppm relative to $\mathrm{SiMe}_{4}\left({ }^{1} \mathrm{H},{ }^{13} \mathrm{C}\right)$, and $85 \% \mathrm{H}_{3} \mathrm{PO}_{4}\left({ }^{31} \mathrm{P}\right)$. Coupling constants $J$ are reported in Hertz $[\mathrm{Hz}]$ as absolute values. Because of high sensitivity of these compounds or the contamination of solvents, the elemental analyses gave unsatisfying results with errors larger than $5 \%$. The high purity of these isolated compounds has been proved mainly by NMR and EPR spectra. UV/vis spectra were measured on Shimadzu UV/vis/NIR UV-3600-spectrometer. Melting point (M. P.) were measured on Buchi M-560 apparatus. 
Preparation of 2a and 2b: RI (1.0 equivalent) in $\mathrm{Et}_{2} \mathrm{O}(1 \mathrm{~mL})$ was added to a stirred solution of $1(86.8 \mathrm{mg}, 0.10 \mathrm{mmol})$ in $\mathrm{Et}_{2} \mathrm{O}(4 \mathrm{~mL})$. After stirring for 5 hours at room temperature, the precipitates were filtered and washed with hexane following by drying in vacuo to afford $\mathbf{2}$ as red powder.

2a (R=Me): $88.2 \mathrm{mg}, 0.087 \mathrm{mmol}, 87.4 \%$ yield. Red crystals were obtained from a saturated toluene/THF solution via slow evaporation. M. P. $>250{ }^{\circ} \mathrm{C} .{ }^{1} \mathrm{H} \mathrm{NMR}\left(\mathrm{CD}_{2} \mathrm{Cl}_{2}\right.$, $400 \mathrm{MHz}): \delta=7.36\left(\mathrm{~m}, 4 \mathrm{H}, \mathrm{C}_{\mathrm{ar}} H\right), 7.13\left(\mathrm{~m}, 8 \mathrm{H}, \mathrm{C}_{\mathrm{ar}} H\right), 4.05\left(\mathrm{~m}, 8 \mathrm{H}, \mathrm{N} H_{2}\right), 3.00(\mathrm{~m}$, $4 \mathrm{H}, \mathrm{CHMe} 2), 2.83(\mathrm{~m}, 4 \mathrm{H}, \mathrm{CHMe} 2), 1.27$ (d, $\left.12 \mathrm{H}, J=6.8 \mathrm{~Hz}, \mathrm{CH}_{3}\right), 1.20$ (d, $12 \mathrm{H}, J$ $\left.=6.8 \mathrm{~Hz}, \mathrm{CH}_{3}\right), 1.14\left(\mathrm{~d}, 12 \mathrm{H}, J=6.8 \mathrm{~Hz}, \mathrm{CH}_{3}\right), 1.10\left(\mathrm{~d}, 12 \mathrm{H}, J=6.8 \mathrm{~Hz}, \mathrm{CH}_{3}\right),-0.07$ $\left(\mathrm{d}, 3 \mathrm{H}, J=4.0 \mathrm{~Hz}, P C H_{3}\right) ;{ }^{13} \mathrm{C}\left\{{ }^{1} \mathrm{H}\right\} \mathrm{NMR}\left(\mathrm{CD}_{2} \mathrm{Cl}_{2}, 100.5 \mathrm{MHz}\right): \delta=162.4\left(\mathrm{t},{ }^{2} J_{\mathrm{PC}}=\right.$ 11.3 Hz, PCC), 147.0 (s, C $\mathrm{ar}), 133.0\left(\mathrm{~s}, \mathrm{C}_{\mathrm{ar}}\right), 130.5\left(\mathrm{~s}, \mathrm{C}_{\mathrm{ar}}\right), 130.2\left(\mathrm{dd},{ }^{1} J_{\mathrm{PC}}=49.8 \mathrm{~Hz}\right.$, $\left.{ }^{1} J_{\mathrm{PC}}=9.0 \mathrm{~Hz}, \mathrm{PCC}\right), 125.5\left(\mathrm{~s}, \mathrm{C}_{\mathrm{ar}}\right), 125.2\left(\mathrm{~s}, \mathrm{C}_{\mathrm{ar}}\right), 52.0\left(\mathrm{NCH}_{2}\right), 28.8,28.8,25.9,25.3$, 23.4, 23.0, $17.4\left(\mathrm{dd},{ }^{1} J_{\mathrm{PC}}=36.0 \mathrm{~Hz},{ }^{3} J_{\mathrm{PC}}=10.1 \mathrm{~Hz}, \mathrm{PCPCH}\right) ;{ }^{31} \mathrm{P} \mathrm{NMR}\left(\mathrm{CD}_{2} \mathrm{Cl}_{2}\right.$, $161.9 \mathrm{MHz}) \delta=434.8\left(\mathrm{~d},{ }^{2} J_{\mathrm{PP}}=67.5 \mathrm{~Hz}\right), 52.7\left(\mathrm{~d},{ }^{2} J_{\mathrm{PP}}=67.5 \mathrm{~Hz}\right) . \mathrm{UV} / \mathrm{Vis}(\mathrm{THF}, \lambda$ (nm) $\varepsilon\left(\mathrm{M}^{-1} \mathrm{~cm}^{-1}\right): 328.1$ (14582), 485.2 (23813).

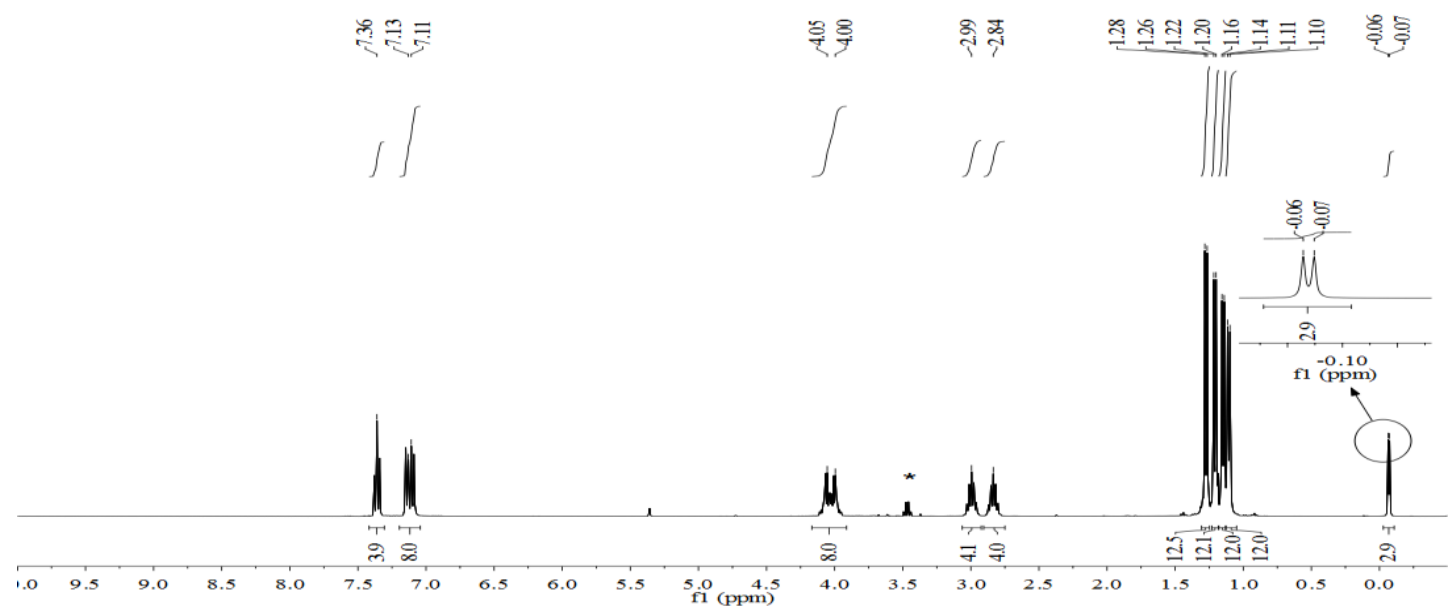

Figure S1. ${ }^{1} \mathrm{H}$ NMR spectrum of $2 \mathbf{a}$ in $\mathrm{CD}_{2} \mathrm{Cl}_{2}$. ${ }^{*}$ Diethyl ether. 


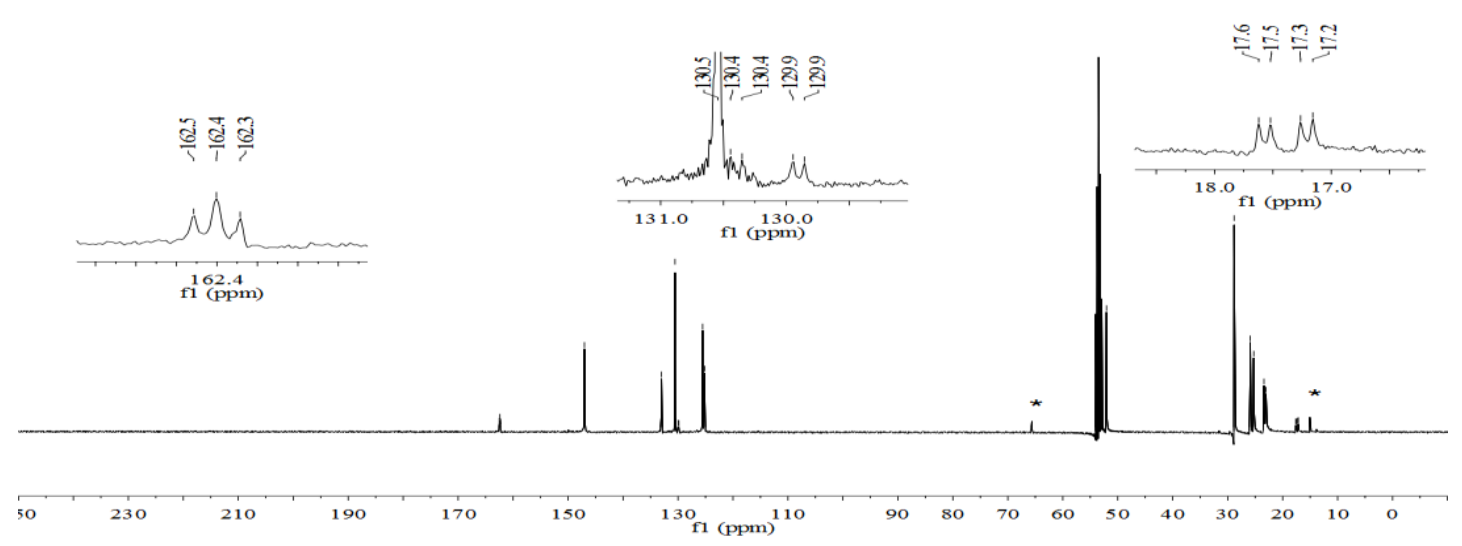

Figure S2. ${ }^{13} \mathrm{C}\left\{{ }^{1} \mathrm{H}\right\}$ NMR spectrum of $\mathbf{2 a}$ in $\mathrm{CD}_{2} \mathrm{Cl}_{2}$. ${ }^{*}$ Diethyl ether.

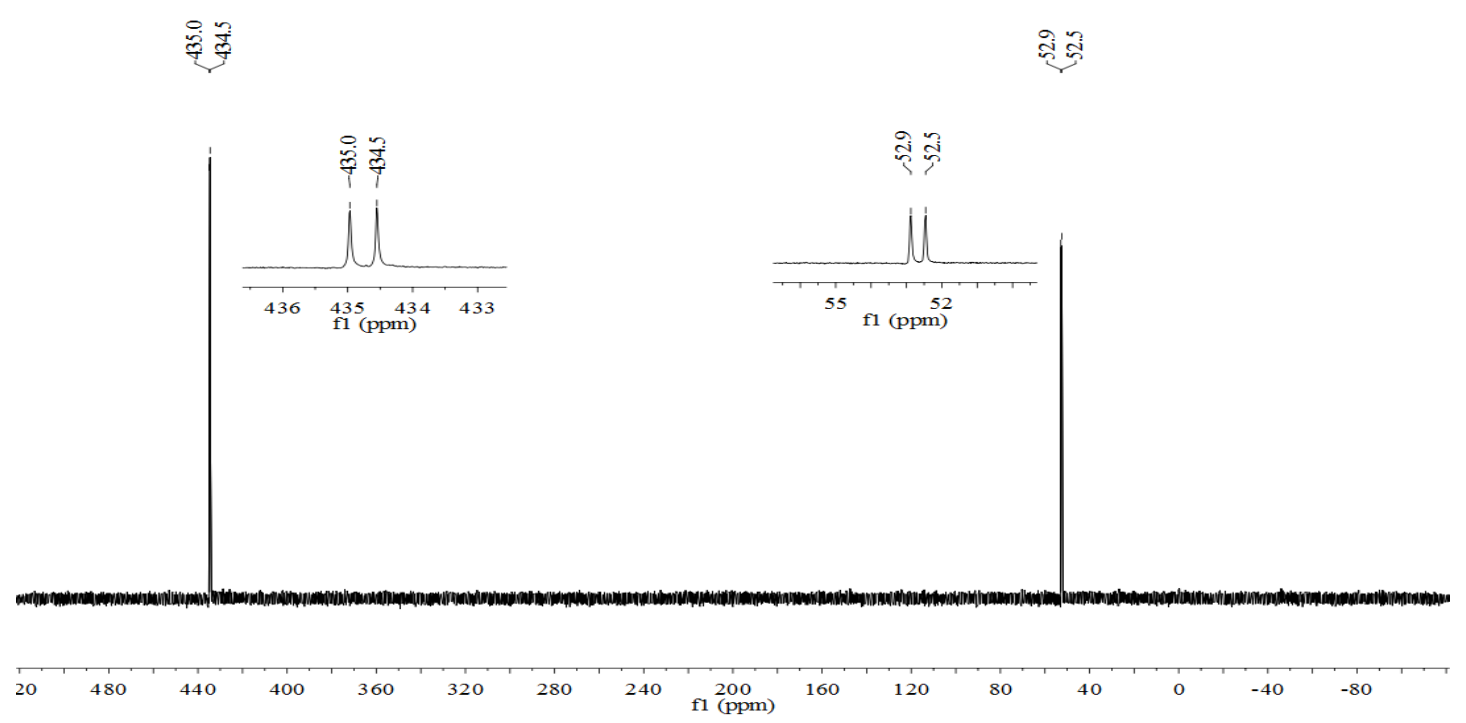

Figure S3. ${ }^{31} \mathrm{P}$ NMR spectrum of $2 \mathrm{a}$ in $\mathrm{CD}_{2} \mathrm{Cl}_{2}$.

2b (R=Et): $92.5 \mathrm{mg}, 0.090 \mathrm{mmol}, 90.4 \%$ yield. M. P. $>250{ }^{\circ} \mathrm{C} .{ }^{1} \mathrm{H} \mathrm{NMR}\left(\mathrm{CD}_{2} \mathrm{Cl}_{2}, 400\right.$ MHz): $\delta=7.37$ (m, 4 H, $\left.\mathrm{C}_{\mathrm{ar}} H\right), 7.13$ (m, $\left.8 \mathrm{H}, \mathrm{C}_{\mathrm{ar}} H\right), 4.08\left(\mathrm{~m}, 4 \mathrm{H}, \mathrm{NH}{ }_{2}\right), 3.98(\mathrm{~m}, 4 \mathrm{H}$, $\mathrm{NH}_{2}$ ), 2.99 (m, $4 \mathrm{H}, \mathrm{C} H \mathrm{Me}_{2}$ ), 2.89 (m, $4 \mathrm{H}, \mathrm{CHMe}$ ), 1.27 (m, 12 H, CH $\left.H_{3}\right), 1.19$ (m, 36 $\left.\mathrm{H}, \mathrm{CH}_{3}\right) 0.43\left(\mathrm{~m}, 3 \mathrm{H}, \mathrm{PCH}_{2} \mathrm{CH}_{3}\right),-0.26\left(\mathrm{~m}, 2 \mathrm{H}, P \mathrm{CH}_{2} \mathrm{CH}_{3}\right) ;{ }^{13} \mathrm{C}\left\{{ }^{1} \mathrm{H}\right\} \mathrm{NMR}\left(\mathrm{CD}_{2} \mathrm{Cl}_{2}\right.$, 100.5 MHz): $\delta=162.1\left(\mathrm{t},{ }^{2} J_{\mathrm{PC}}=11.3 \mathrm{~Hz}, \mathrm{PCC}\right), 147.1\left(\mathrm{~s}, \mathrm{C}_{\mathrm{ar}}\right), 146.9\left(\mathrm{~s}, \mathrm{C}_{\mathrm{ar}}\right), 133.1(\mathrm{~s}$, $\mathrm{C}_{\mathrm{ar}}$ ), 130.6 (s, $\mathrm{C}_{\mathrm{ar}}$ ), 125.7 (overlapped with other peaks, $\mathrm{PCC}$ ), 125.4 (s, $\mathrm{C}_{\mathrm{ar}}$ ), 125.2 (s, $\left.\mathrm{C}_{\mathrm{ar}}\right), 52.2\left(\mathrm{NCH}_{2}\right), 28.8,28.8,26.2,25.6,23.3,22.9,22.8,19.6\left(\mathrm{dd},{ }^{1} J_{\mathrm{PC}}=36.7 \mathrm{~Hz},{ }^{3} J_{\mathrm{PC}}\right.$ $\left.=7.2 \mathrm{~Hz}, \mathrm{PCPCH} \mathrm{CH}_{3}\right), 7.8\left(\mathrm{~d},{ }^{2} \mathrm{JCC}_{\mathrm{PC}}=4.8 \mathrm{~Hz}, \mathrm{PCPCH} \mathrm{CH}_{3}\right) ;{ }^{31} \mathrm{P} \mathrm{NMR}\left(\mathrm{CD}_{2} \mathrm{Cl}_{2}, 161.9\right.$ 
$\mathrm{MHz}) \delta=439.2\left(\mathrm{~d},{ }^{2} J_{\mathrm{PP}}=71.7 \mathrm{~Hz}\right), 66.7\left(\mathrm{dd},{ }^{2} J_{\mathrm{PP}}=71.7 \mathrm{~Hz},{ }^{2} J_{\mathrm{PH}}=7.6 \mathrm{~Hz}\right) . \mathrm{UV} / \mathrm{V}$ is (THF, $\lambda(\mathrm{nm}) \varepsilon\left(\mathrm{M}^{-1} \mathrm{~cm}^{-1}\right): 320.9$ (9745), 483.1 (27734).

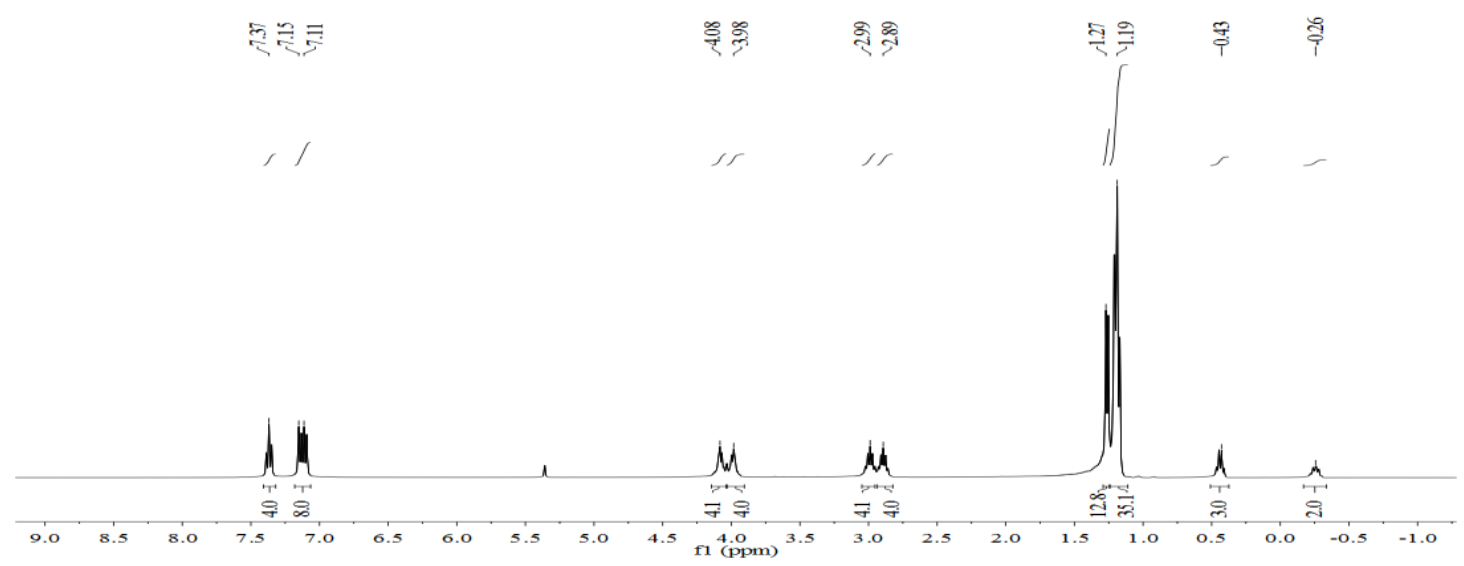

Figure S4. ${ }^{1} \mathrm{H}$ NMR spectrum of $\mathbf{2 b}$ in $\mathrm{CD}_{2} \mathrm{Cl}_{2}$. *Diethyl ether.

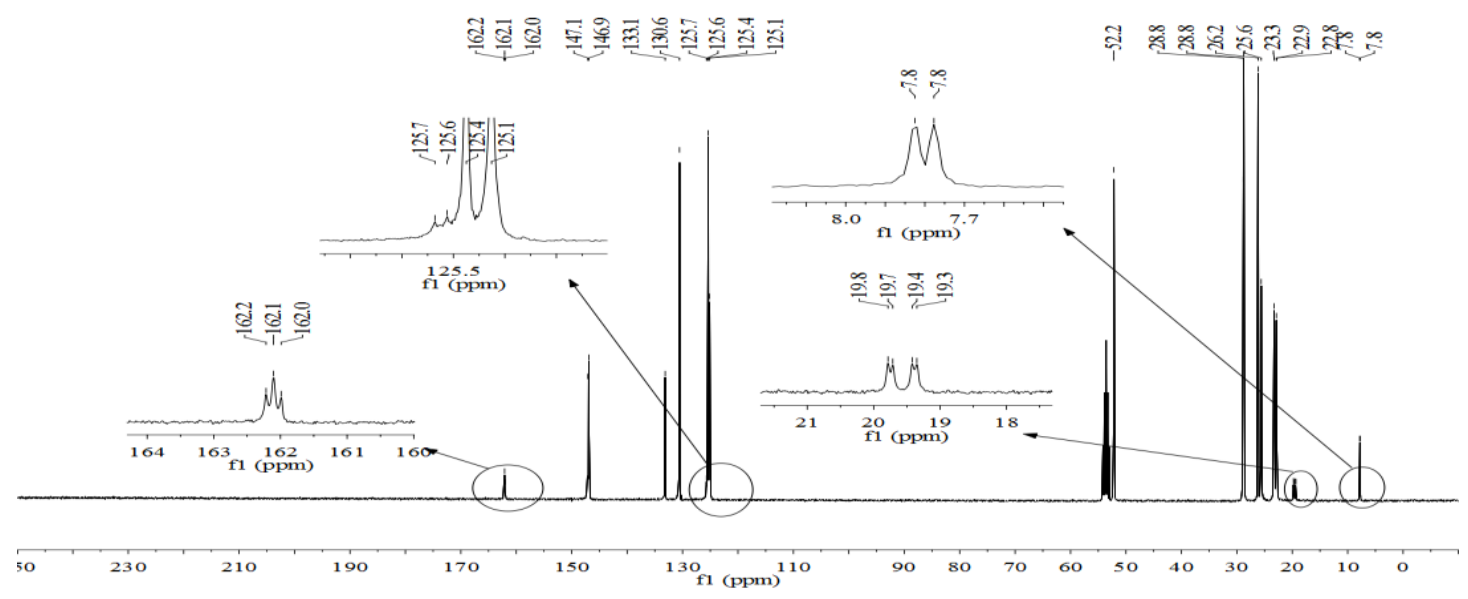

Figure S5. ${ }^{13} \mathrm{C}\left\{{ }^{1} \mathrm{H}\right\}$ NMR spectrum of $\mathbf{2 b}$ in $\mathrm{CD}_{2} \mathrm{Cl}_{2}$. $*$ Diethyl ether.

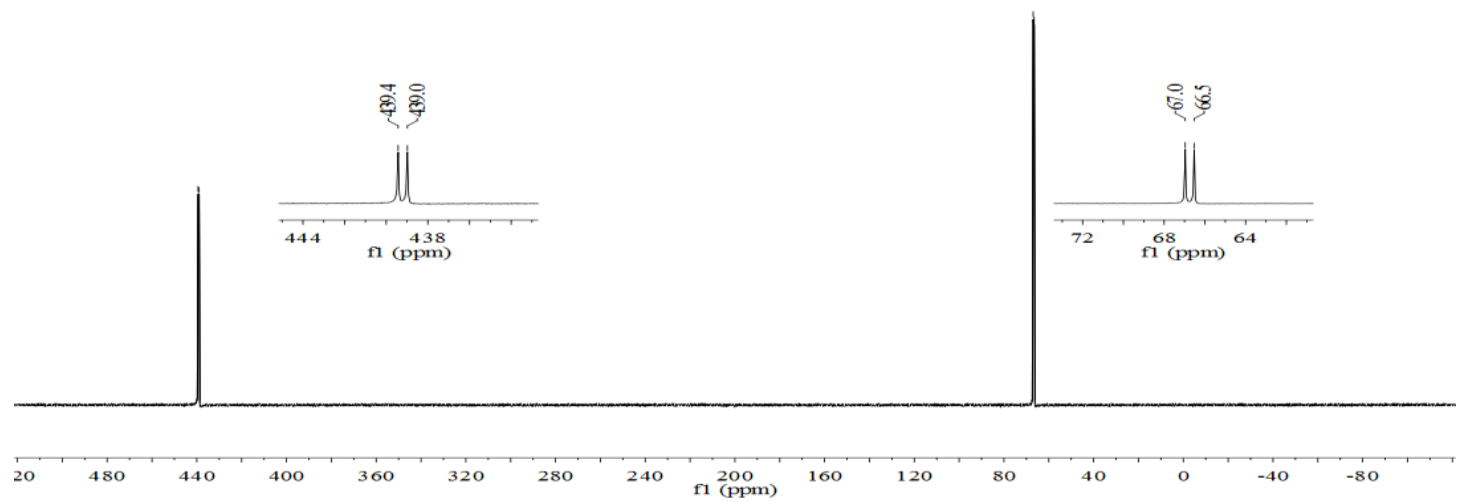

Figure S6. ${ }^{31} \mathrm{P}\left\{{ }^{1} \mathrm{H}\right\}$ NMR spectrum of $\mathbf{2 b}$ in $\mathrm{CD}_{2} \mathrm{Cl}_{2}$. 


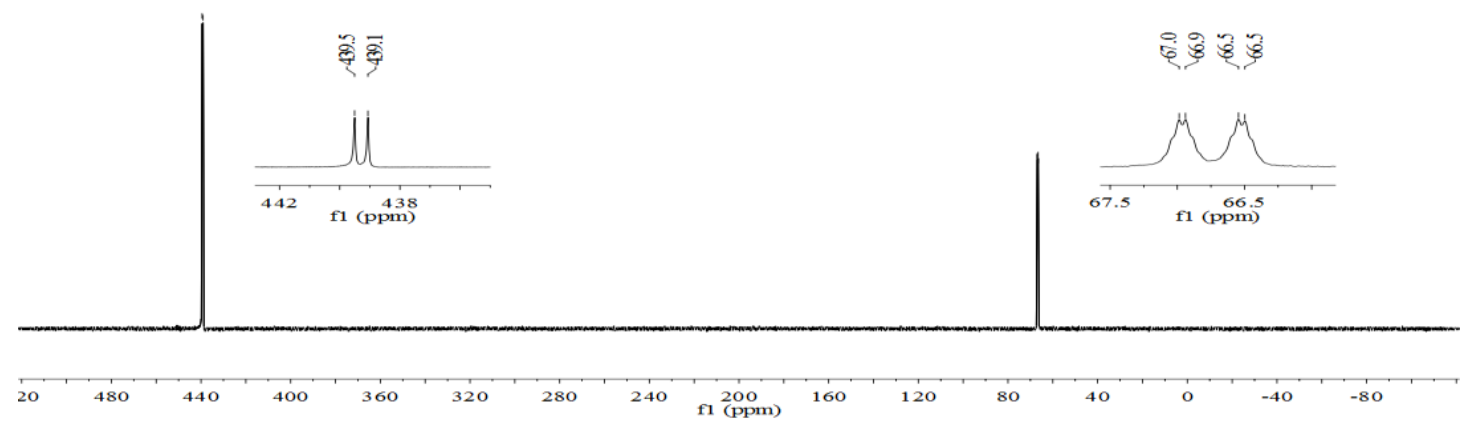

Figure S7. ${ }^{31} \mathrm{P}$ NMR spectrum of $\mathbf{2 b}$ in $\mathrm{CD}_{2} \mathrm{Cl}_{2}$.

Preparation of $2 \mathrm{c}:{ }^{i} \mathrm{BuI}$ (5.0 equivalent) in THF $(1 \mathrm{~mL})$ was added to a stirred solution of $1(86.8 \mathrm{mg}, 0.10 \mathrm{mmol})$ in THF $(4 \mathrm{~mL})$. After stirring for 12 hours at room temperature, the solvents were removed under reduced pressure. The remaining residue was washed with toluene and dried in vacuo to afford $\mathbf{2 c}$ as red powder $(75.2 \mathrm{mg}, 0.073$ mmol, $72.5 \%$ yield). M. P. > $250{ }^{\circ} \mathrm{C} .{ }^{1} \mathrm{H}$ NMR $\left(\mathrm{CD}_{2} \mathrm{Cl}_{2}, 400 \mathrm{MHz}\right): \delta=7.33(\mathrm{~m}, 4 \mathrm{H}$, $\left.\mathrm{C}_{\mathrm{ar}} H\right), 7.10\left(\mathrm{~m}, 8 \mathrm{H}, \mathrm{C}_{\mathrm{ar}} H\right), 4.00\left(\mathrm{~m}, 8 \mathrm{H}, \mathrm{NH} \mathrm{H}_{2}\right), 3.01$ (m, $\left.4 \mathrm{H}, \mathrm{CHMe}\right), 2.85$ (m, $4 \mathrm{H}$, $\mathrm{CHMe} 2), 1.20\left(\mathrm{~m}, 48 \mathrm{H}, \mathrm{CH}_{3}\right), 0.55\left(\mathrm{~m}, 1 \mathrm{H}, \mathrm{PCH}\left(\mathrm{CH}_{3}\right)_{2}\right), 0.40\left(\mathrm{~m}, 6 \mathrm{H}, \mathrm{PCH}\left(\mathrm{CH}_{3}\right)_{2}\right)$; ${ }^{13} \mathrm{C}\left\{{ }^{1} \mathrm{H}\right\} \mathrm{NMR}\left(\mathrm{CD}_{2} \mathrm{Cl}_{2}, 100.5 \mathrm{MHz}\right): \delta=162.9\left(\mathrm{t},{ }^{2} J_{\mathrm{PC}}=11.1 \mathrm{~Hz}, \mathrm{PCC}\right), 150.4\left(\mathrm{~s}, \mathrm{C}_{\mathrm{ar}}\right)$, $147.3\left(\mathrm{~s}, \mathrm{C}_{\mathrm{ar}}\right), 146.9$ (s, C $\left.\mathrm{ar}\right), 133.7\left(\mathrm{~s}, \mathrm{C}_{\mathrm{ar}}\right), 130.9\left(\mathrm{~s}, \mathrm{C}_{\mathrm{ar}}\right), 129.2\left(\mathrm{dd},{ }^{1} J_{\mathrm{PC}}=49.1 \mathrm{~Hz},{ }^{1} J_{\mathrm{PC}}\right.$ = $12.2 \mathrm{~Hz}, \mathrm{PCC}), 128.9\left(\mathrm{~s}, \mathrm{C}_{\mathrm{ar}}\right), 128.5\left(\mathrm{~s}, \mathrm{C}_{\mathrm{ar}}\right), 126.0\left(\mathrm{~s}, \mathrm{C}_{\mathrm{ar}}\right), 125.7\left(\mathrm{~s}, \mathrm{C}_{\mathrm{ar}}\right), 125.6(\mathrm{~s}$, $\left.\mathrm{C}_{\mathrm{ar}}\right), 52.8\left(\mathrm{NCH}_{2}\right), 42.3\left(\mathrm{dd},{ }^{1} J_{\mathrm{PC}}=35.1 \mathrm{~Hz},{ }^{3} J_{\mathrm{PC}}=8.6 \mathrm{~Hz}, \mathrm{PCPCH}\left(\mathrm{CH}_{3}\right)_{2}\right), 29.2,29.2$, 26.6, 26.4, 24.6, 24.6, 24.5, 23.8, 23.4; ${ }^{31} \mathrm{P} \mathrm{NMR}\left(\mathrm{CD}_{2} \mathrm{Cl}_{2}, 161.9 \mathrm{MHz}\right) \delta=439.1(\mathrm{~d}$, $\left.{ }^{2} J_{\mathrm{PP}}=69.1 \mathrm{~Hz}\right), 61.1\left(\mathrm{~d},{ }^{2} J_{\mathrm{PP}}=69.1 \mathrm{~Hz}\right) . \mathrm{UV} / \mathrm{Vis}\left(\mathrm{THF}, \lambda(\mathrm{nm}) \varepsilon\left(\mathrm{M}^{-1} \mathrm{~cm}^{-1}\right): 317.3\right.$ (15426), 486.7 (35940). 


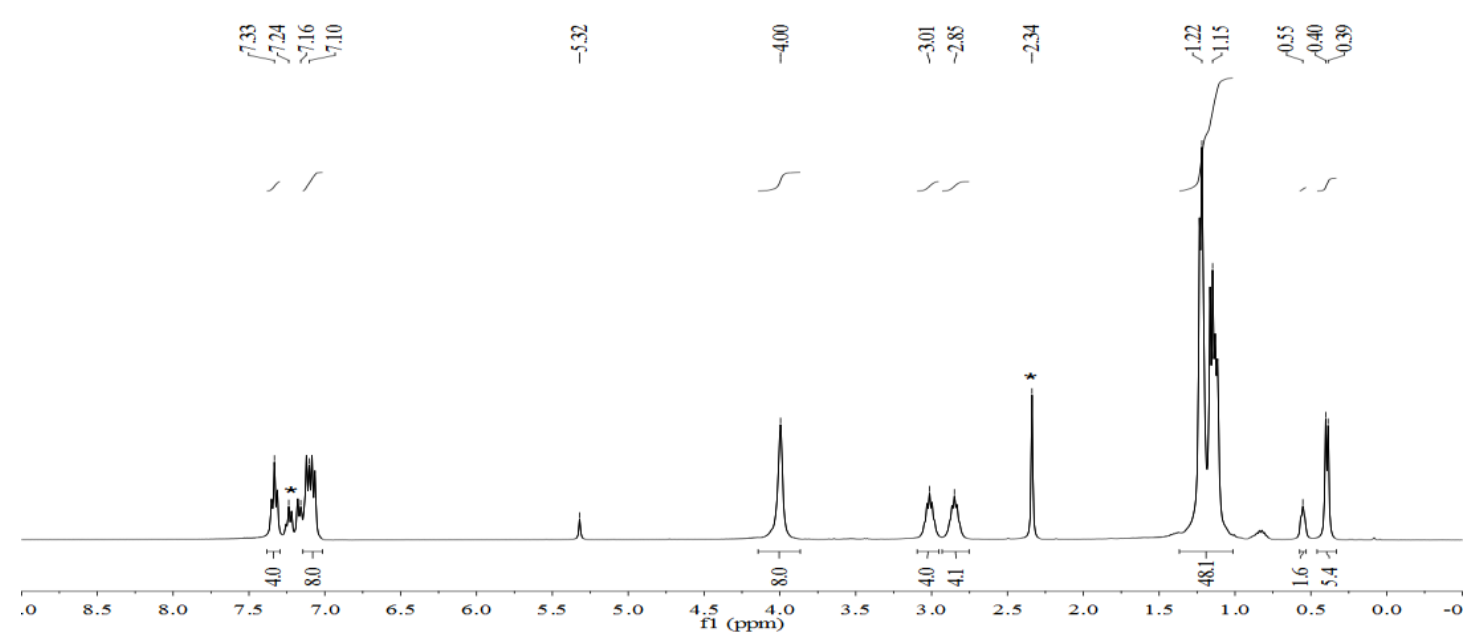

Figure S8. ${ }^{1} \mathrm{H}$ NMR spectrum of $2 \mathrm{c}$ in $\mathrm{CD}_{2} \mathrm{Cl}_{2}$. $*$ Toluene.

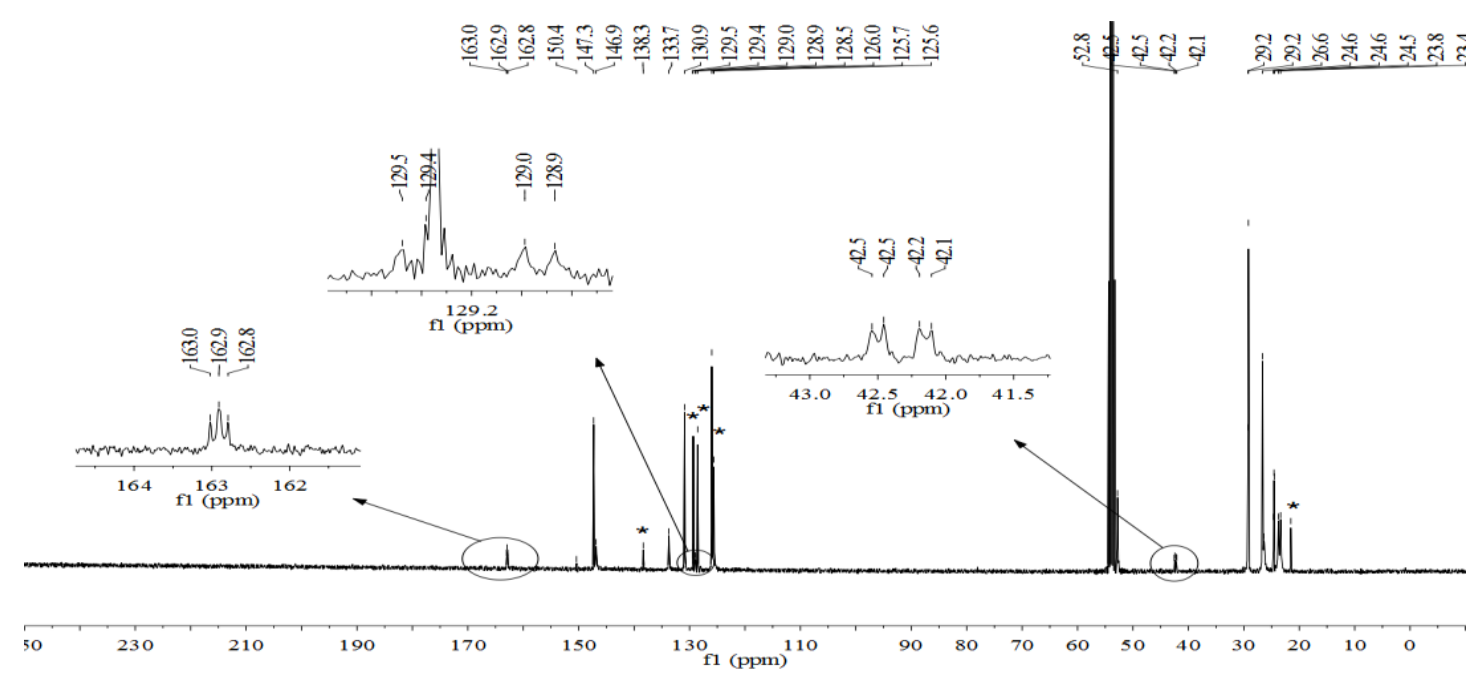

Figure S9. ${ }^{13} \mathrm{C}\left\{{ }^{1} \mathrm{H}\right\}$ NMR spectrum of $2 \mathrm{c}$ in $\mathrm{CD}_{2} \mathrm{Cl}_{2}$. *Toluene.

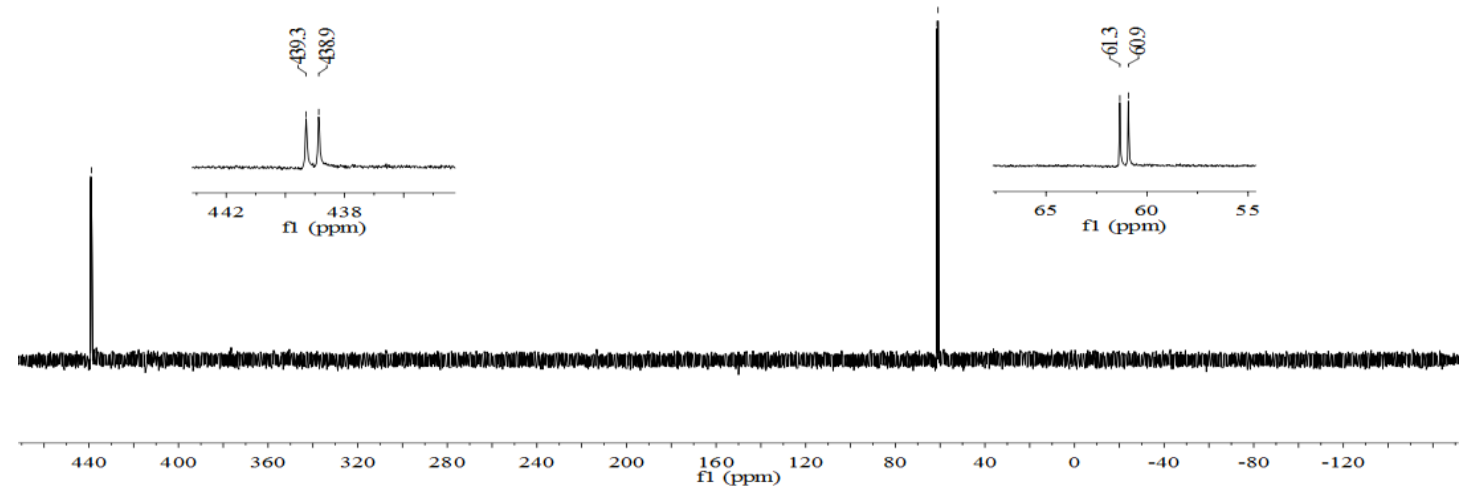

Figure S10. ${ }^{31} \mathrm{P}$ NMR spectrum of $2 \mathrm{c}$ in $\mathrm{CD}_{2} \mathrm{Cl}_{2}$. 
Preparation of 3: $\mathrm{Me}_{2} \mathrm{Zn}$ (2.0 equivalent, $1.2 \mathrm{M}$ in toluene) in THF (2 mL) was added dropwise to a stirred solution of $2(0.10 \mathrm{mmol})$ in THF $(4 \mathrm{~mL})$. After stirring for 12 hours at room temperature, the solvent was removed under reduced pressure. The remaining residue was extracted with diethyl ether and the solvent was removed under reduced pressure. The remaining solid was washed with acetonitrile and dried in vacuo affording 3 as white powder.

3a $(\mathrm{R}=\mathrm{Me})$ : $42.6 \mathrm{mg}, 0.047 \mathrm{mmol}, 47.5 \%$ yield. White crystals were obtained from a saturated diethyl ether/hexane solution via slow evaporation. M. P. > $250{ }^{\circ} \mathrm{C}$. ${ }^{1} \mathrm{H}$ NMR $\left(\mathrm{C}_{6} \mathrm{D}_{6}, 400 \mathrm{MHz}\right): \delta=7.13\left(\mathrm{~m}, 8 \mathrm{H}, \mathrm{C}_{\mathrm{ar}} H\right), 698\left(\mathrm{~m}, 4 \mathrm{H}, \mathrm{C}_{\mathrm{ar}} H\right), 3.66\left(\mathrm{~m}, 4 \mathrm{H}, \mathrm{NH}_{2}\right)$, $3.47\left(\mathrm{~m}, 4 \mathrm{H}, \mathrm{NH} H_{2}\right), 3.27\left(\mathrm{~m}, 8 \mathrm{H}, \mathrm{CHMe}_{2}\right), 1.52\left(\mathrm{~d}, 12 \mathrm{H}, J=6.4 \mathrm{~Hz}, \mathrm{CH}_{3}\right), 1.33(\mathrm{~m}$, $\left.\left.24 \mathrm{H}, \mathrm{CH}_{3}\right), 1.11\left(\mathrm{~d}, 12 \mathrm{H}, J=6.4 \mathrm{~Hz}, \mathrm{CH}_{3}\right), 0.53(\mathrm{~s}, 6 \mathrm{H}, \mathrm{PCH})_{3}\right){ }^{13} \mathrm{C}\left\{{ }^{1} \mathrm{H}\right\} \mathrm{NMR}\left(\mathrm{C}_{6} \mathrm{D}_{6}\right.$, 100.5 MHz): $\delta=152.0\left(\mathrm{t},{ }^{2} J_{\mathrm{PC}}=20.2 \mathrm{~Hz}, \mathrm{PCC}\right), 149.6\left(\mathrm{~s}, \mathrm{C}_{\mathrm{ar}}\right), 147.2\left(\mathrm{~s}, \mathrm{C}_{\mathrm{ar}}\right), 139.3(\mathrm{~s}$, $\left.\mathrm{C}_{\mathrm{ar}}\right), 127.8\left(\mathrm{~s}, \mathrm{C}_{\mathrm{ar}}\right), 124.2\left(\mathrm{~s}, \mathrm{C}_{\mathrm{ar}}\right), 123.8\left(\mathrm{~s}, \mathrm{C}_{\mathrm{ar}}\right), 64.3$ (t, $\left.{ }^{1} \mathrm{~J}_{\mathrm{PC}}=3.9 \mathrm{~Hz}, \mathrm{PCC}\right), 52.1$ $\left(\mathrm{NCH}_{2}\right), 28.3,27.9,26.0,25.8,23.4,23.3,23.3,22.8,22.2\left(\mathrm{dd},{ }^{1} J_{\mathrm{PC}}=45.7 \mathrm{~Hz},{ }^{3} J_{\mathrm{PC}}=\right.$ $5.3 \mathrm{~Hz}, \mathrm{PCPCH} 3) ;{ }^{31} \mathrm{P}$ NMR $\left(\mathrm{C}_{6} \mathrm{D}_{6}, 161.9 \mathrm{MHz}\right) \delta=67.3(\mathrm{~s}) . \mathrm{UV} / \mathrm{Vis}(\mathrm{THF}, \lambda(\mathrm{nm}) \varepsilon$ $\left(\mathrm{M}^{-1} \mathrm{~cm}^{-1}\right): 286.6$ (47334).

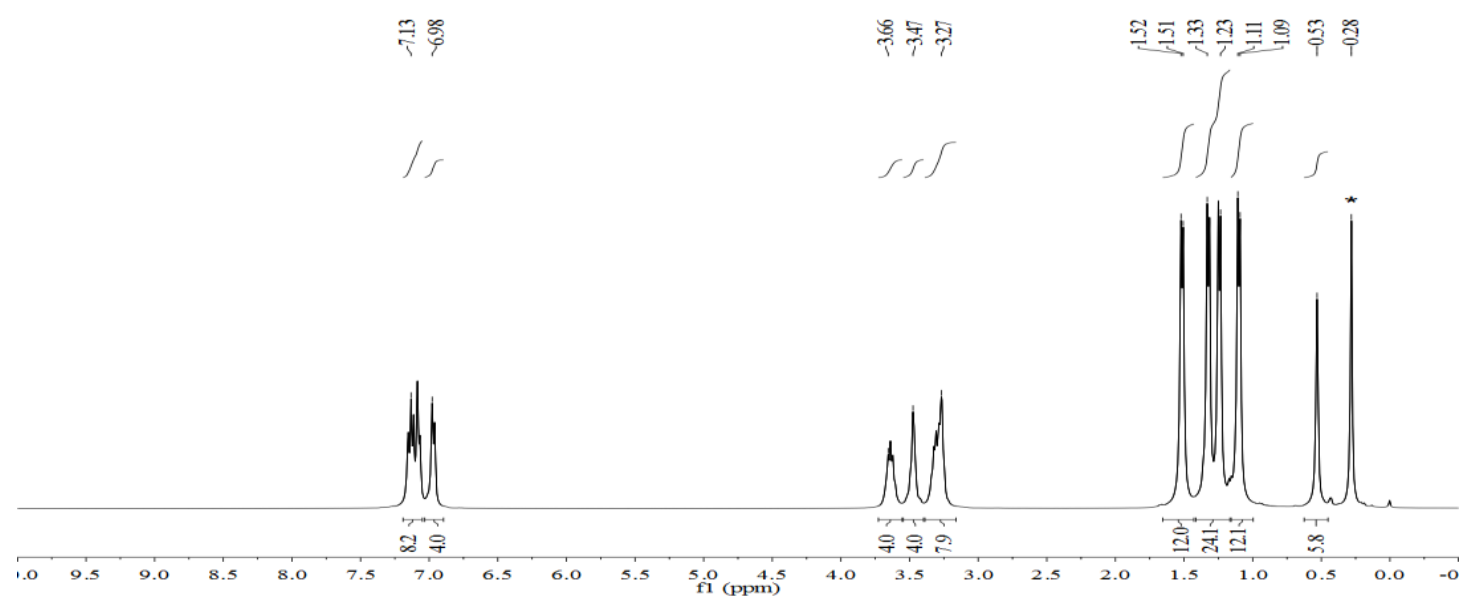

Figure S11. ${ }^{1} \mathrm{H}$ NMR spectrum of $3 \mathbf{a}$ in $\mathrm{C}_{6} \mathrm{D}_{6}$. ${ }^{*}$ Silicone grease. 


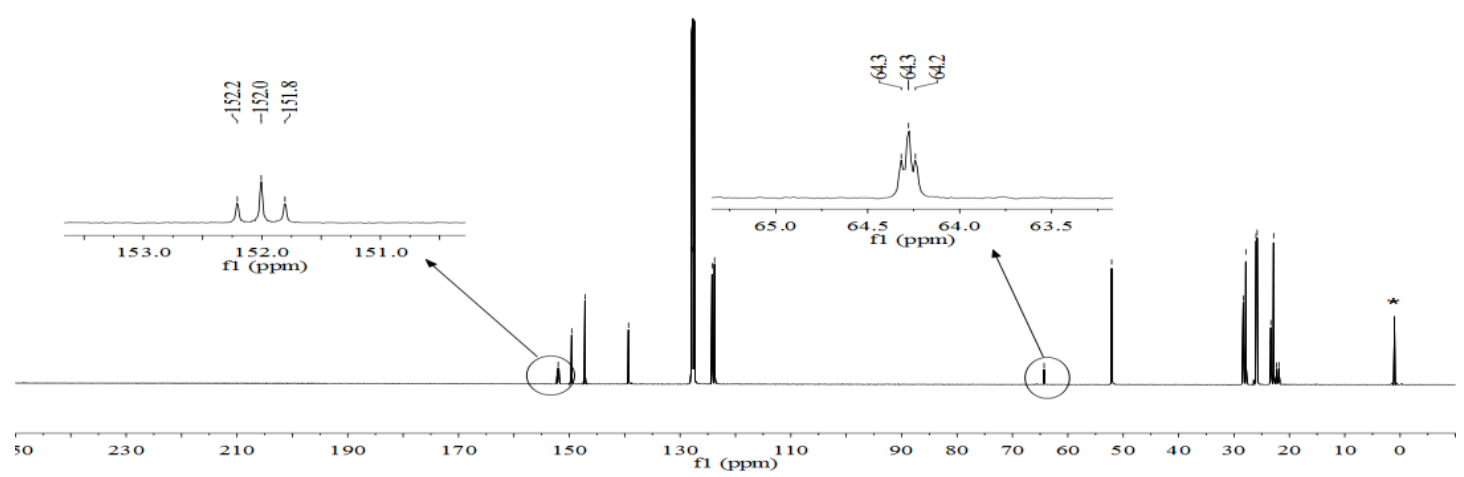

Figure S12. ${ }^{13} \mathrm{C}\left\{{ }^{1} \mathrm{H}\right\}$ NMR spectrum of 3a in $\mathrm{C}_{6} \mathrm{D}_{6}$. ${ }^{*}$ Silicone grease.

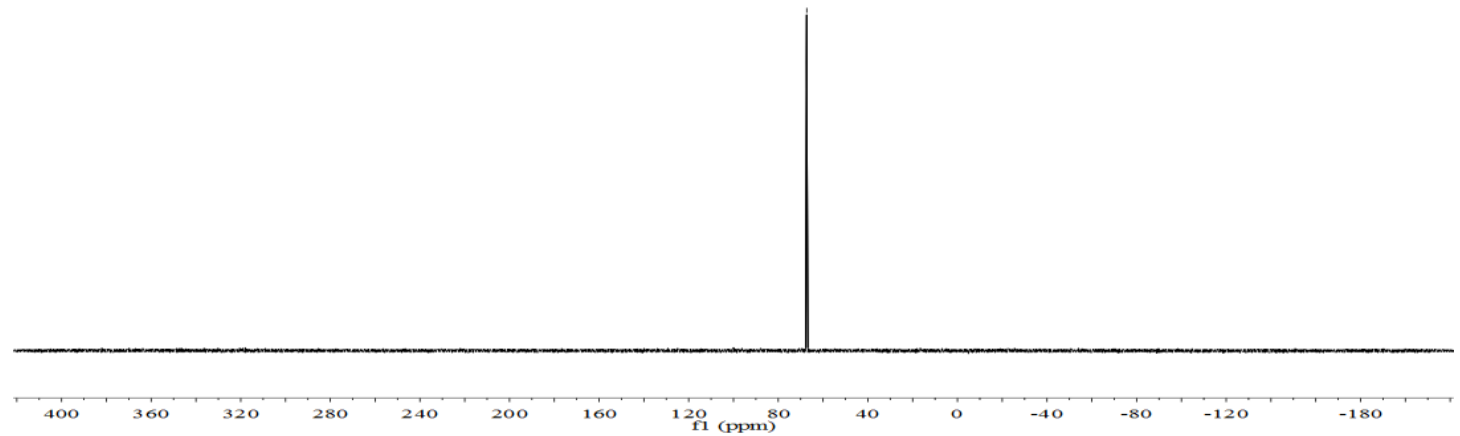

Figure S13. ${ }^{31} \mathrm{P}\left\{{ }^{1} \mathrm{H}\right\}$ NMR spectrum of $\mathbf{3 a}$ in $\mathrm{C}_{6} \mathrm{D}_{6}$.

a)

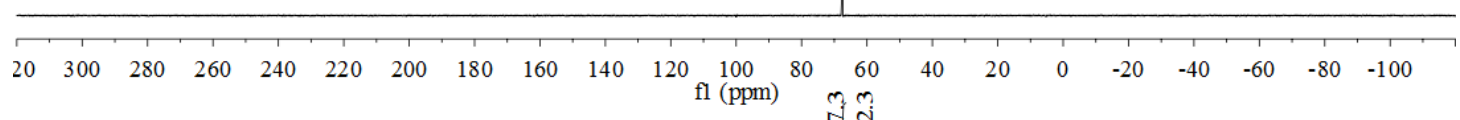

b)
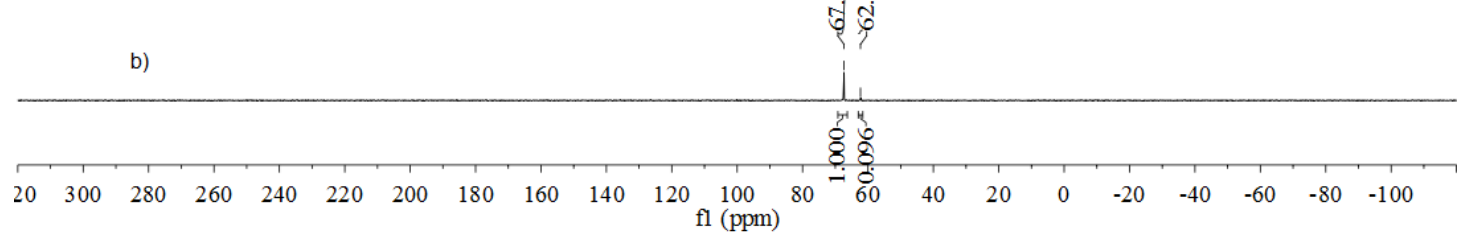

c)

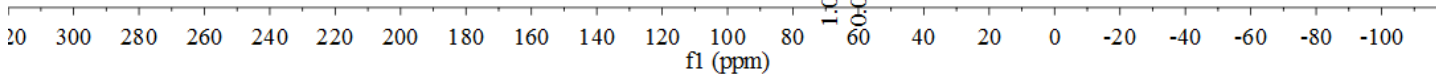

d)

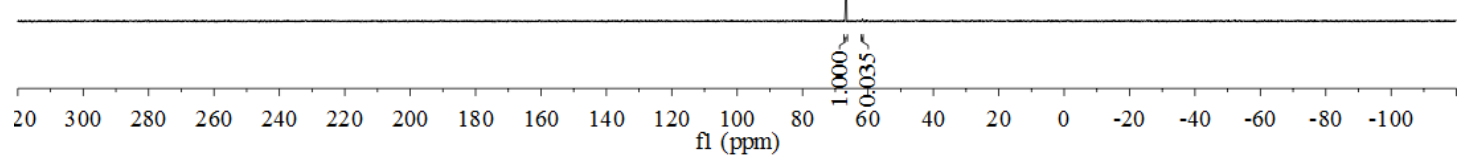


Figure S14. ${ }^{31} \mathrm{P}\left\{{ }^{1} \mathrm{H}\right\}$ NMR spectrum of $\mathbf{3 a}$ in toluene (a), after heating to $110{ }^{\circ} \mathrm{C}$ for 5 hours (b), and then after stay at room temperature for 2 days (c) and 8 days (d).

3b (R=Et): $49.8 \mathrm{mg}, 0.055 \mathrm{mmol}, 54.7 \%$ yield. White crystals were obtained from a saturated diethyl ether/hexane solution via slow evaporation. M. P. > $250{ }^{\circ} \mathrm{C} .{ }^{1} \mathrm{H}$ NMR $\left(\mathrm{C}_{6} \mathrm{D}_{6}, 400 \mathrm{MHz}\right): \delta=7.11\left(\mathrm{~m}, 8 \mathrm{H}, \mathrm{C}_{\mathrm{ar}} H\right), 6.98\left(\mathrm{~m}, 4 \mathrm{H}, \mathrm{C}_{\mathrm{ar}} H\right), 3.72\left(\mathrm{~m}, 4 \mathrm{H}, \mathrm{NH}_{2}\right)$, $3.48\left(\mathrm{~m}, 12 \mathrm{H}, \mathrm{NH}_{2}, \mathrm{CHMe}\right.$ ), 1.57 (m, $\left.12 \mathrm{H}, \mathrm{CH}_{3}\right), 1.34$ (m, $\left.24 \mathrm{H}, \mathrm{CH}_{3}\right), 1.10$ (m, 12 $\left.\mathrm{H}, \mathrm{CH}_{3}\right), 0.88\left(\mathrm{~m}, 2 \mathrm{H}, \mathrm{PCH}_{2} \mathrm{CH}_{3}\right), 0.56\left(\mathrm{~m}, 3 \mathrm{H}, \mathrm{PCH}_{2} \mathrm{CH}_{3}\right), 0.42\left(\mathrm{~d},{ }^{2} J_{\mathrm{PH}}=4.0 \mathrm{~Hz}, 2\right.$ $\left.\mathrm{H}, \mathrm{PCH}{ }_{3}\right) ;{ }^{13} \mathrm{C}\left\{{ }^{1} \mathrm{H}\right\} \mathrm{NMR}\left(\mathrm{C}_{6} \mathrm{D}_{6}, 100.5 \mathrm{MHz}\right): \delta=152.2\left(\mathrm{t},{ }^{2} J_{\mathrm{PC}}=20.5 \mathrm{~Hz}, \mathrm{PCC}\right), 149.4$ $\left(\mathrm{d}, J_{\mathrm{PC}}=3.3 \mathrm{~Hz}, \mathrm{C}_{\mathrm{ar}}\right), 149.3\left(\mathrm{~d}, J_{\mathrm{PC}}=3.3 \mathrm{~Hz}, \mathrm{C}_{\mathrm{ar}}\right), 147.0\left(\mathrm{~d}, J_{\mathrm{PC}}=4.2 \mathrm{~Hz}, \mathrm{C}_{\mathrm{ar}}\right), 139.7(\mathrm{~s}$, $\mathrm{C}_{\mathrm{ar}}$ ), $139.4\left(\mathrm{~s}, \mathrm{C}_{\mathrm{ar}}\right), 127.6\left(\mathrm{~s}, \mathrm{C}_{\mathrm{ar}}\right.$ ), 124.3 (d, $\left.J_{\mathrm{PC}}=4.4 \mathrm{~Hz}, \mathrm{C}_{\mathrm{ar}}\right), 123.7$ ( $\mathrm{s}, \mathrm{C}_{\mathrm{ar}}$ ), 63.7 (t, $\left.{ }^{1} J_{\mathrm{PC}}=3.0 \mathrm{~Hz}, \mathrm{PCC}\right), 52.3\left(\mathrm{~s}, \mathrm{NCH}_{2}\right), 52.0\left(\mathrm{~s}, \mathrm{NCH}_{2}\right), 28.4,28.3,28.3,28.2,27.9,27.8$, 26.2, 26.1, 25.9, $23.3\left(\mathrm{dd},{ }^{1} J_{\mathrm{PC}}=24.8 \mathrm{~Hz},{ }^{3} J_{\mathrm{PC}}=9.3 \mathrm{~Hz}, \mathrm{PCPCH}_{3}\right), 22.7,22.6,20.5(\mathrm{~d}$, $\left.{ }^{1} J_{\mathrm{PC}}=39.3 \mathrm{~Hz}, \mathrm{PCH}_{2} \mathrm{CH}_{3}\right), 11.4\left(\mathrm{~d},{ }^{2} J_{\mathrm{PC}}=16.7 \mathrm{~Hz}, \mathrm{PCH}_{2} \mathrm{CH}_{3}\right) ;{ }^{31} \mathrm{P} \mathrm{NMR}\left(\mathrm{C}_{6} \mathrm{D}_{6}, 161.9\right.$ $\mathrm{MHz}) \delta=85.0\left(\mathrm{~m},{ }^{2} J_{\mathrm{PP}}=18.9 \mathrm{~Hz}, \mathrm{PCH}_{3}\right), 70.6\left(\mathrm{~d},{ }^{2} J_{\mathrm{PP}}=18.9 \mathrm{~Hz}, \mathrm{PCH}_{2} \mathrm{CH}_{3}\right) . \mathrm{UV} / \mathrm{V}$ is (THF, $\lambda(\mathrm{nm}) \varepsilon\left(\mathrm{M}^{-1} \mathrm{~cm}^{-1}\right): 286.1(28050)$.

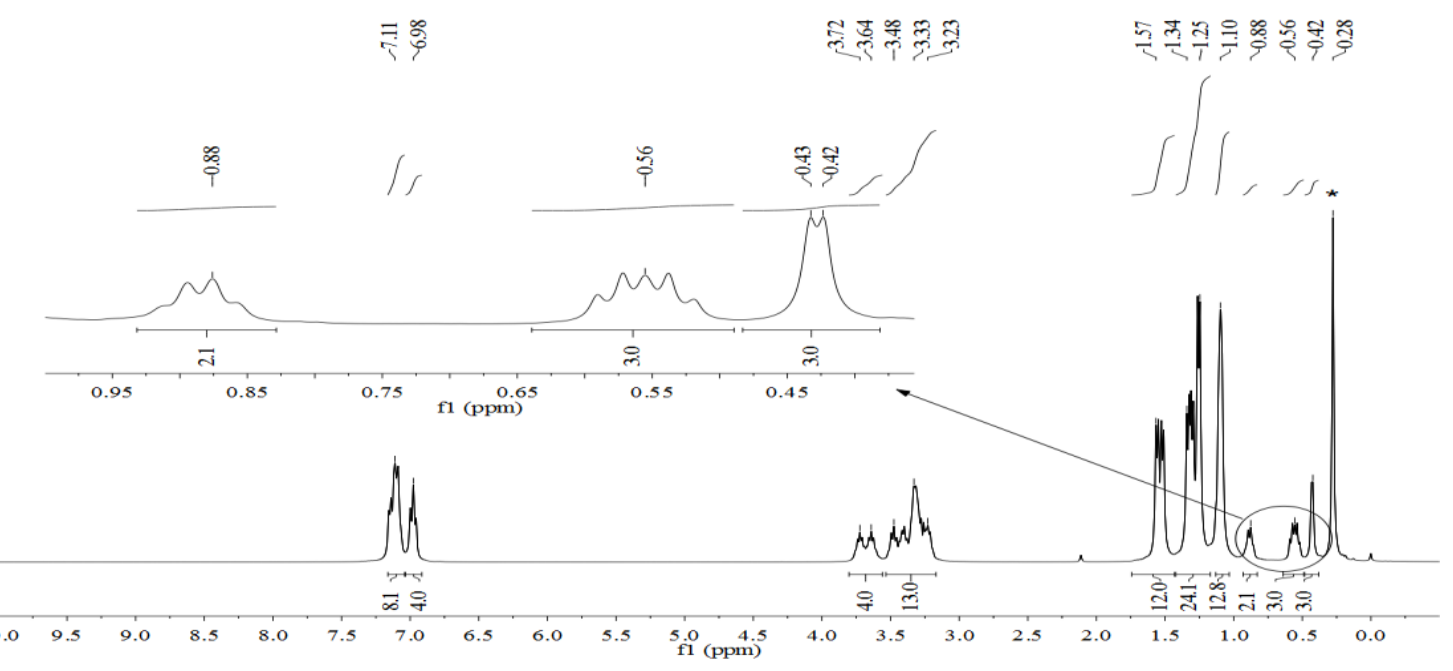

Figure S15. ${ }^{1} \mathrm{H}$ NMR spectrum of $3 \mathbf{b}$ in $\mathrm{C}_{6} \mathrm{D}_{6}$. ${ }^{*}$ Silicone grease. 


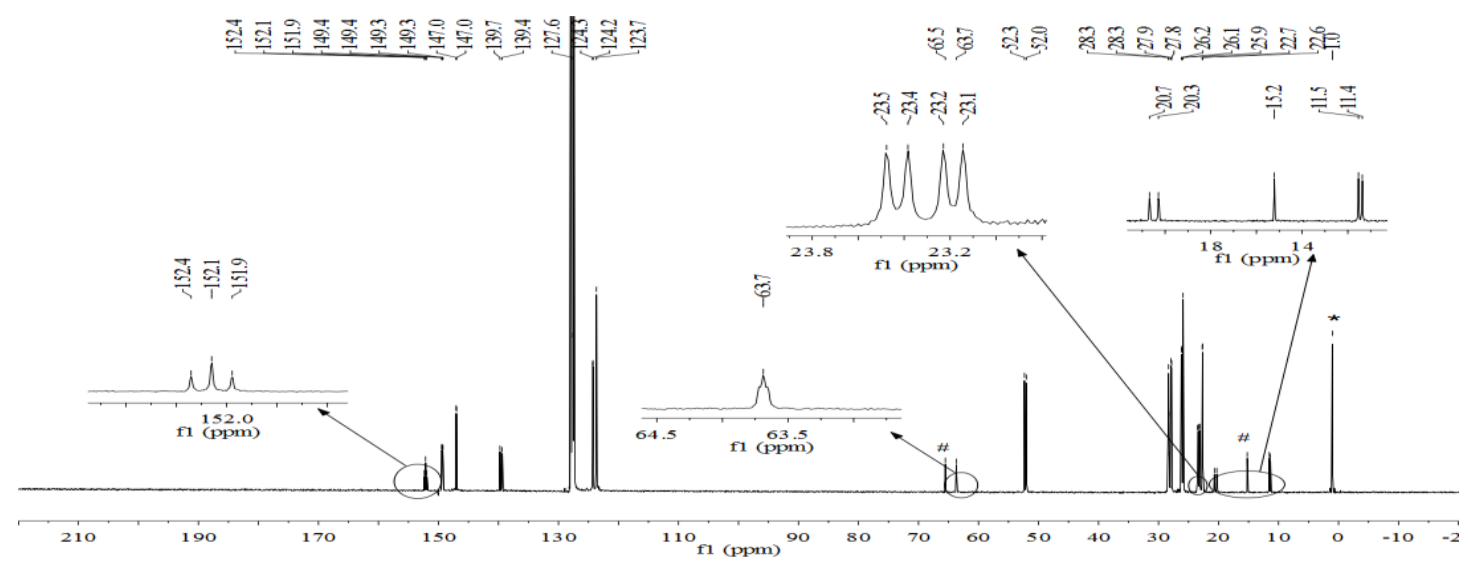

Figure S16. ${ }^{13} \mathrm{C}\left\{{ }^{1} \mathrm{H}\right\}$ NMR spectrum of $\mathbf{3 b}$ in $\mathrm{C}_{6} \mathrm{D}_{6}$. ${ }^{*}$ Silicone grease, ${ }^{\#}$ Diethyl ether.

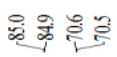

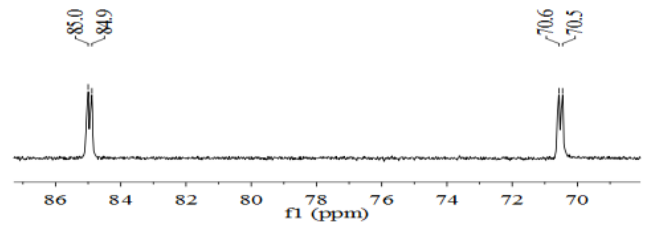

Figure S17. ${ }^{31} \mathrm{P}\left\{{ }^{1} \mathrm{H}\right\}$ NMR spectrum of $3 \mathbf{b}$ in $\mathrm{C}_{6} \mathrm{D}_{6}$.

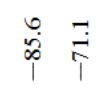

a)

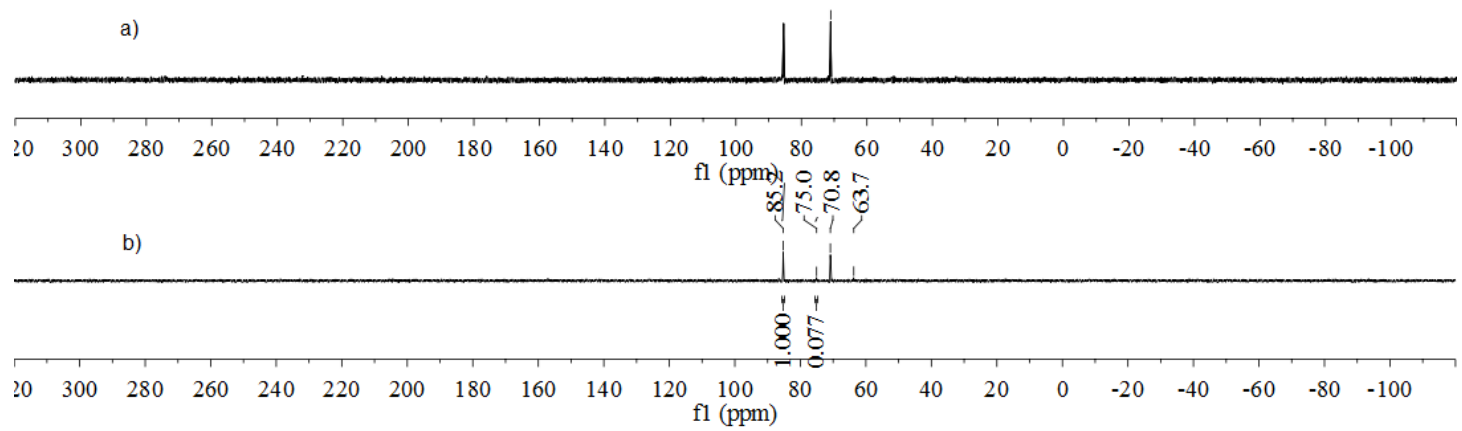

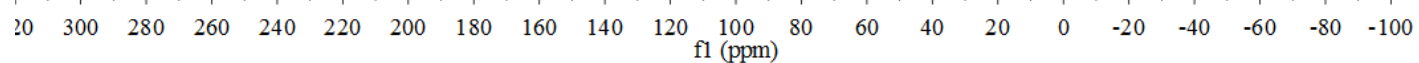

$\begin{array}{llllllllllllllllllllll}20 & 300 & 280 & 260 & 240 & 220 & 200 & 180 & 160 & 140 & 120 & 100 & 100 & 80 & 40 & 20 & 0 & -20 & -40 & -60 & -80 & -100\end{array}$ 
Figure S18. ${ }^{31} \mathrm{P}\left\{{ }^{1} \mathrm{H}\right\} \mathrm{NMR}$ spectrum of $\mathbf{3 b}$ in toluene (a), after heating to $110{ }^{\circ} \mathrm{C}$ for 5 hours (b), and then after stay at room temperature for 1 day (c) and 2 days (d).

3c $\left(\mathrm{R}={ }^{i} \mathrm{Bu}\right): 47.9 \mathrm{mg}, 0.052 \mathrm{mmol}, 51.8 \%$ yield. White crystals were obtained from a saturated diethyl ether/acetonitrile solution via slow evaporation. M. P. > $250{ }^{\circ} \mathrm{C} .{ }^{1} \mathrm{H}$ NMR $\left(\mathrm{C}_{6} \mathrm{D}_{6}, 400 \mathrm{MHz}\right): \delta=7.09\left(\mathrm{~m}, 8 \mathrm{H}, \mathrm{C}_{\mathrm{ar}} H\right), 6.99\left(\mathrm{~m}, 4 \mathrm{H}, \mathrm{C}_{\mathrm{ar}} H\right), 3.84(\mathrm{~m}, 2 \mathrm{H}$, $\left.\mathrm{NH} H_{2}\right), 3.72\left(\mathrm{~m}, 2 \mathrm{H}, \mathrm{NH}_{2}\right), 3.48\left(\mathrm{~m}, 14 \mathrm{H}, \mathrm{NH} H_{2}\right), 3.34(\mathrm{~m}, 6 \mathrm{H}, \mathrm{CHMe}), 3.17(\mathrm{~m}, 2 \mathrm{H}$, $\left.\mathrm{CHMe}_{2}\right), 1.68\left(\mathrm{~d}, 6 \mathrm{H}, J=6.4 \mathrm{~Hz}, \mathrm{CH}_{3}\right), 1.52\left(\mathrm{~d}, 6 \mathrm{H}, J=6.4 \mathrm{~Hz}, \mathrm{CH}_{3}\right), 1.39$ (d, $6 \mathrm{H}, J$ $\left.=6.4 \mathrm{~Hz}, \mathrm{CH}_{3}\right), 1.29\left(\mathrm{~m}, 18 \mathrm{H}, \mathrm{CH}_{3}\right), 1.11\left(\mathrm{~d}, 12 \mathrm{H}, J=6.8 \mathrm{~Hz}, \mathrm{CH}_{3}\right), 0.89(\mathrm{~m}, 1 \mathrm{H}$, $\mathrm{PCHMe} 2), 0.54\left(\mathrm{~d}, 3 \mathrm{H},{ }^{2} \mathrm{~J}_{\mathrm{PH}}=4.0 \mathrm{~Hz}, \mathrm{PCH}_{3}\right), 0.44\left(\mathrm{~d}, 6 \mathrm{H},{ }^{3} J_{\mathrm{PH}}=6.0 \mathrm{~Hz}, \mathrm{PCHMe} 2\right)$; ${ }^{13} \mathrm{C}\left\{{ }^{1} \mathrm{H}\right\}$ NMR $\left(\mathrm{C}_{6} \mathrm{D}_{6}, 100.5 \mathrm{MHz}\right): \delta=152.6\left(\mathrm{t},{ }^{2} J_{\mathrm{PC}}=21.6 \mathrm{~Hz}, \mathrm{PCC}\right), 149.1\left(\mathrm{~d}, J_{\mathrm{PC}}=\right.$ $\left.3.1 \mathrm{~Hz}, \mathrm{C}_{\mathrm{ar}}\right), 148.9\left(\mathrm{~d}, J_{\mathrm{PC}}=2.5 \mathrm{~Hz}, \mathrm{C}_{\mathrm{ar}}\right), 146.7\left(\mathrm{~d}, J_{\mathrm{PC}}=6.7 \mathrm{~Hz}, \mathrm{C}_{\mathrm{ar}}\right), 139.7\left(\mathrm{~s}, \mathrm{C}_{\mathrm{ar}}\right)$, $139.4\left(\mathrm{~s}, \mathrm{C}_{\mathrm{ar}}\right), 127.6\left(\mathrm{~s}, \mathrm{C}_{\mathrm{ar}}\right), 124.5\left(\mathrm{~d}, J_{\mathrm{PC}}=10.3 \mathrm{~Hz}, \mathrm{C}_{\mathrm{ar}}\right), 124.0\left(\mathrm{~s}, \mathrm{C}_{\mathrm{ar}}\right), 123.8\left(\mathrm{~s}, \mathrm{C}_{\mathrm{ar}}\right)$, $64.5\left(\mathrm{t},{ }^{1} J_{\mathrm{PC}}=3.6 \mathrm{~Hz}, \mathrm{PCC}\right), 52.7\left(\mathrm{~s}, \mathrm{NCH}_{2}\right), 52.1\left(\mathrm{~s}, \mathrm{NCH}_{2}\right), 46.7\left(\mathrm{~d},{ }^{1} J_{\mathrm{PC}}=16.8 \mathrm{~Hz}\right.$, $\left.\mathrm{PC} \mathrm{Me}_{2}\right), 28.3$, 28.3, 28.2 2 28.2 2 27.8, 27.5, 26.5, 25.9, 25.9, 24.6, 24.5, 23.6, 23.5, 23.5, 23.4, 22.7, 22.7, 23.3, $20.1\left(\mathrm{~d},{ }^{1} J_{\mathrm{PC}}=38.4 \mathrm{~Hz}, \mathrm{PCH}_{3}\right) ;{ }^{31} \mathrm{P} \mathrm{NMR}\left(\mathrm{C}_{6} \mathrm{D}_{6}, 161.9 \mathrm{MHz}\right) \delta$ $=84.3\left(\mathrm{~m},{ }^{2} J_{\mathrm{PP}}=15.7 \mathrm{~Hz}\right), 83.8\left(\mathrm{~d},{ }^{2} J_{\mathrm{PP}}=15.7 \mathrm{~Hz}, \mathrm{PCHMe} 2\right) . \mathrm{UV} / \mathrm{Vis}(\mathrm{THF}, \lambda(\mathrm{nm}) \varepsilon$ $\left(\mathrm{M}^{-1} \mathrm{~cm}^{-1}\right): 287.3(36256)$.

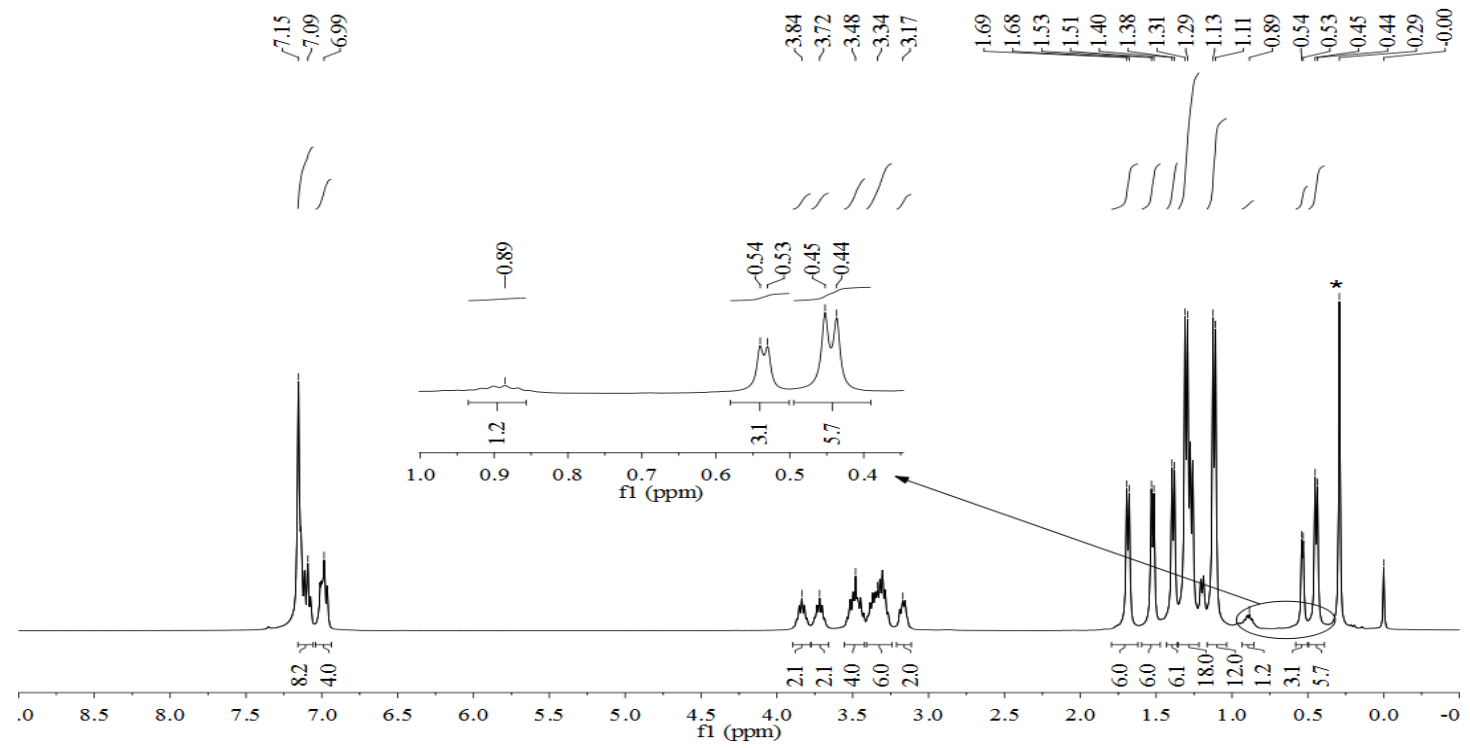

Figure S19. ${ }^{1} \mathrm{H}$ NMR spectrum of $3 \mathrm{c}$ in $\mathrm{C}_{6} \mathrm{D}_{6} .{ }^{*}$ Silicone grease. 


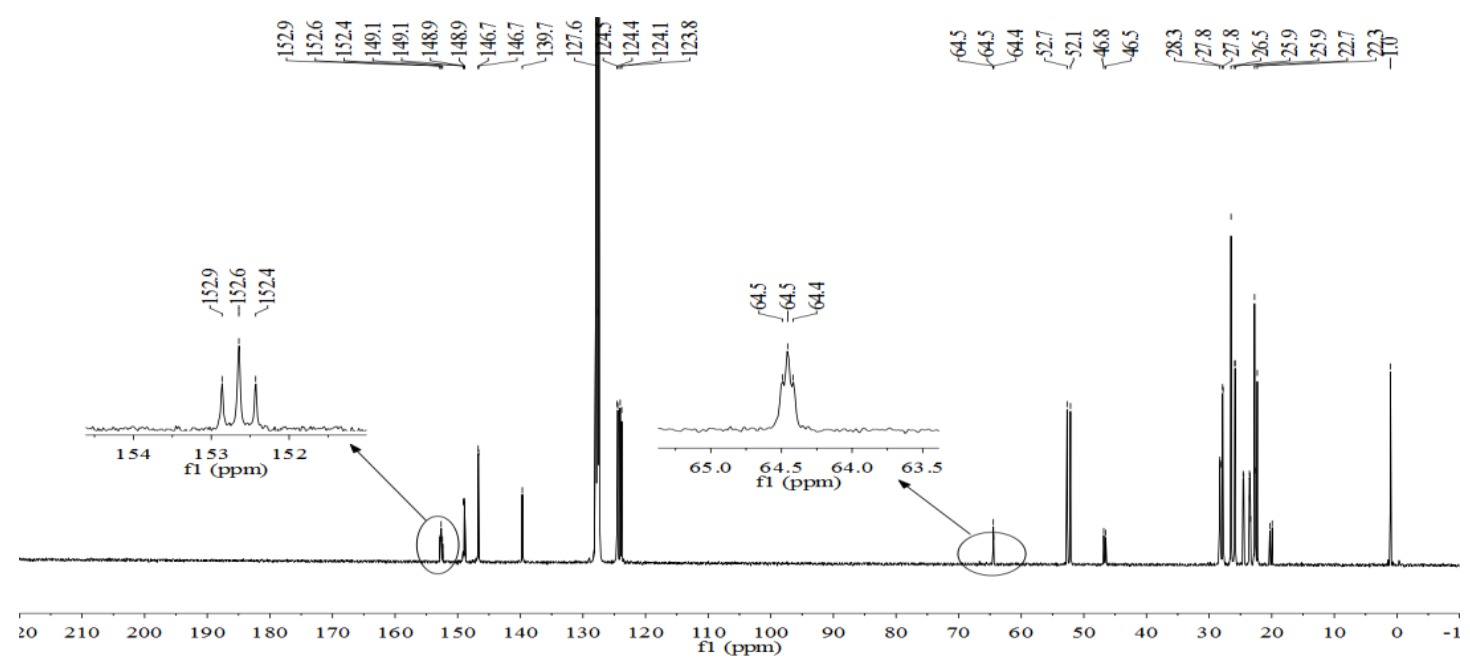

Figure S20. ${ }^{13} \mathrm{C}\left\{{ }^{1} \mathrm{H}\right\}$ NMR spectrum of $3 \mathbf{c}$ in $\mathrm{C}_{6} \mathrm{D}_{6}$.

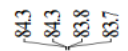

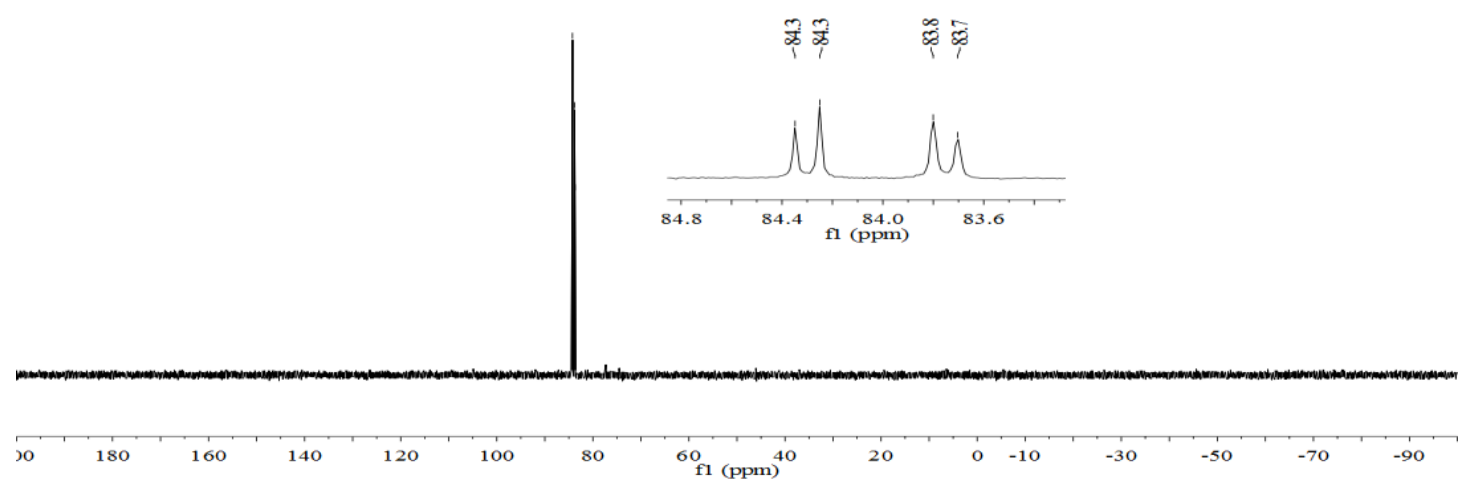

Figure S21. ${ }^{31} \mathrm{P}\left\{{ }^{1} \mathrm{H}\right\}$ NMR spectrum of $3 \mathbf{c}$ in $\mathrm{C}_{6} \mathrm{D}_{6}$.

Preparation of $3^{\bullet+}(\mathbf{B A R F})$ : $\mathrm{FcBAr}^{\mathrm{F}}$ (1.0 equivalent) was added in portions to a stirred solution of $3(0.05 \mathrm{mmol})$ in diethyl ether $(4 \mathrm{~mL})$ at room temperature. After stirring for 1.0 hour, the solvent was removed under reduced pressure. The remaining solid was washed with hexane and dried in vacuo affording deep red powder. Depp red crystals of these radicals were obtained from a diethyl ether and hexane solution via slow evaporation.

$\mathbf{3 a}^{\bullet+}\left(\mathrm{BAr}{ }^{\mathrm{F}}\right)(\mathrm{R}=\mathrm{Me}, \mathrm{Me}): 81.8 \mathrm{mg}, 0.046 \mathrm{mmol}, 92.9 \%$ yield. $\mathrm{M} . \mathrm{P} .=182{ }^{\circ} \mathrm{C} . \mathrm{UV} / \mathrm{V}$ is (THF, $\lambda(\mathrm{nm}) \varepsilon\left(\mathrm{M}^{-1} \mathrm{~cm}^{-1}\right): 279.8$ (20533), 333.2 (8775), 537.2 (1197). 
$\mathbf{3 b}^{\bullet+}\left(\mathrm{BAr}{ }^{\mathrm{F}}\right)(\mathrm{R}=\mathrm{Me}, \mathrm{Et}): 80.3 \mathrm{mg}, 0.045 \mathrm{mmol}, 90.5 \%$ yield. $\mathrm{M} . \mathrm{P} .=175^{\circ} \mathrm{C}$. UV/Vis (THF, $\lambda(\mathrm{nm}) \varepsilon\left(\mathrm{M}^{-1} \mathrm{~cm}^{-1}\right): 291.3$ (21839), 546.3 (1720).

$\left.\mathbf{3 c}^{\bullet+}(\mathrm{BAr})^{\mathrm{F}}\right)(\mathrm{R}=\mathrm{Me}, \mathrm{iPr}): 83.5 \mathrm{mg}, 0.047 \mathrm{mmol}, 93.4 \%$ yield. $\mathrm{M} . \mathrm{P} .=178{ }^{\circ} \mathrm{C} . \mathrm{UV} / \mathrm{V}$ is (THF, $\lambda(\mathrm{nm}) \varepsilon\left(\mathrm{M}^{-1} \mathrm{~cm}^{-1}\right): 279.2$ (16943), 489.7 (1995).

\section{S2. X-Ray Diffraction Studies}

These data can be obtained free of charge via http://www.ccdc.cam.ac.uk/cgibin/catreq.cgi, or by emailing data_request@ccdc.cam.ac.uk. The CCDC reference numbers are 2051355 (2a), 2051356 (3a), 2051357 (3b), 2051358(3c), 2051359

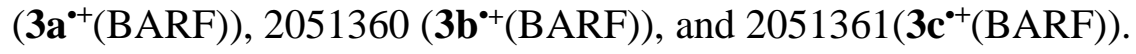

Table $S 1$. The summary of crystal data and structure refinement

\begin{tabular}{|c|c|c|c|c|}
\hline Compounds & 2a & $3 a$ & $3 \mathbf{b}$ & $3 c$ \\
\hline $\mathrm{CCDC}$ & 2051355 & 2051356 & 2051357 & 2051358 \\
\hline Empirical formula & $\mathrm{C}_{64} \mathrm{H}_{87} \mathrm{IN}_{4} \mathrm{P}_{2}$ & $\mathrm{C}_{62} \mathrm{H}_{92} \mathrm{~N}_{4} \mathrm{OP}_{2}$ & $\mathrm{C}_{120} \mathrm{H}_{173} \mathrm{~N}_{8} \mathrm{OP}_{4}$ & $\mathrm{C}_{128} \mathrm{H}_{189} \mathrm{~N}_{9} \mathrm{OP}_{4}$ \\
\hline Formula weight & 1101.21 & 971.33 & 1867.53 & 1993.75 \\
\hline Temperature/K & $150.00(10)$ & $150.00(10)$ & $150.00(10)$ & $100.0(2)$ \\
\hline Crystal system & monoclinic & monoclinic & monoclinic & monoclinic \\
\hline Space group & $\mathrm{P} 2{ }_{1} / \mathrm{n}$ & $\mathrm{P} 2{ }_{1} / \mathrm{n}$ & $\mathrm{C} 2 / \mathrm{c}$ & $\mathrm{C} 2 / \mathrm{c}$ \\
\hline $\mathrm{a} / \AA ̊$ & $15.06790(10)$ & $14.56630(10)$ & $20.7420(3)$ & $21.2086(3)$ \\
\hline $\mathrm{b} / \AA ̊ \AA$ & $25.6212(2)$ & $18.17850(10)$ & $15.0561(3)$ & $15.1217(2)$ \\
\hline $\mathrm{c} / \AA ̊$ & $15.72590(10)$ & $22.03690(10)$ & $37.5279(6)$ & $37.4035(6)$ \\
\hline$\alpha /^{\circ}$ & 90 & 90 & 90 & 90 \\
\hline$\beta /{ }^{\circ}$ & $96.3580(10)$ & $93.5260(10)$ & $103.276(2)$ & $97.9190(10)$ \\
\hline$\gamma /{ }^{\circ}$ & 90 & 90 & 90 & 90 \\
\hline Volume $/ \AA^{3}$ & $6033.76(7)$ & $5824.18(6)$ & $11406.5(3)$ & $11881.3(3)$ \\
\hline $\mathrm{Z}$ & 4 & 4 & 4 & 4 \\
\hline$\rho_{\text {calc }} \mathrm{g} / \mathrm{cm}^{3}$ & 1.212 & 1.108 & 1.087 & 1.115 \\
\hline$\mu / \mathrm{mm}^{-1}$ & 4.970 & 0.988 & 0.986 & 0.977 \\
\hline $\mathrm{F}(000)$ & 2328.0 & 2120.0 & 4068.0 & 4352.0 \\
\hline Crystal size $/ \mathrm{mm}^{3}$ & $0.2 \times 0.15 \times 0.15$ & $0.21 \times 0.18 \times 0.13$ & $0.5 \times 0.1 \times 0.1$ & $0.15 \times 0.12 \times 0.1$ \\
\hline Radiation & $\mathrm{CuK} \alpha(\lambda=1.54184)$ & $\operatorname{CuK} \alpha(\lambda=1.54184)$ & $\mathrm{CuK} \alpha(\lambda=1.54184)$ & $\mathrm{CuK} \alpha(\lambda=1.54184)$ \\
\hline
\end{tabular}




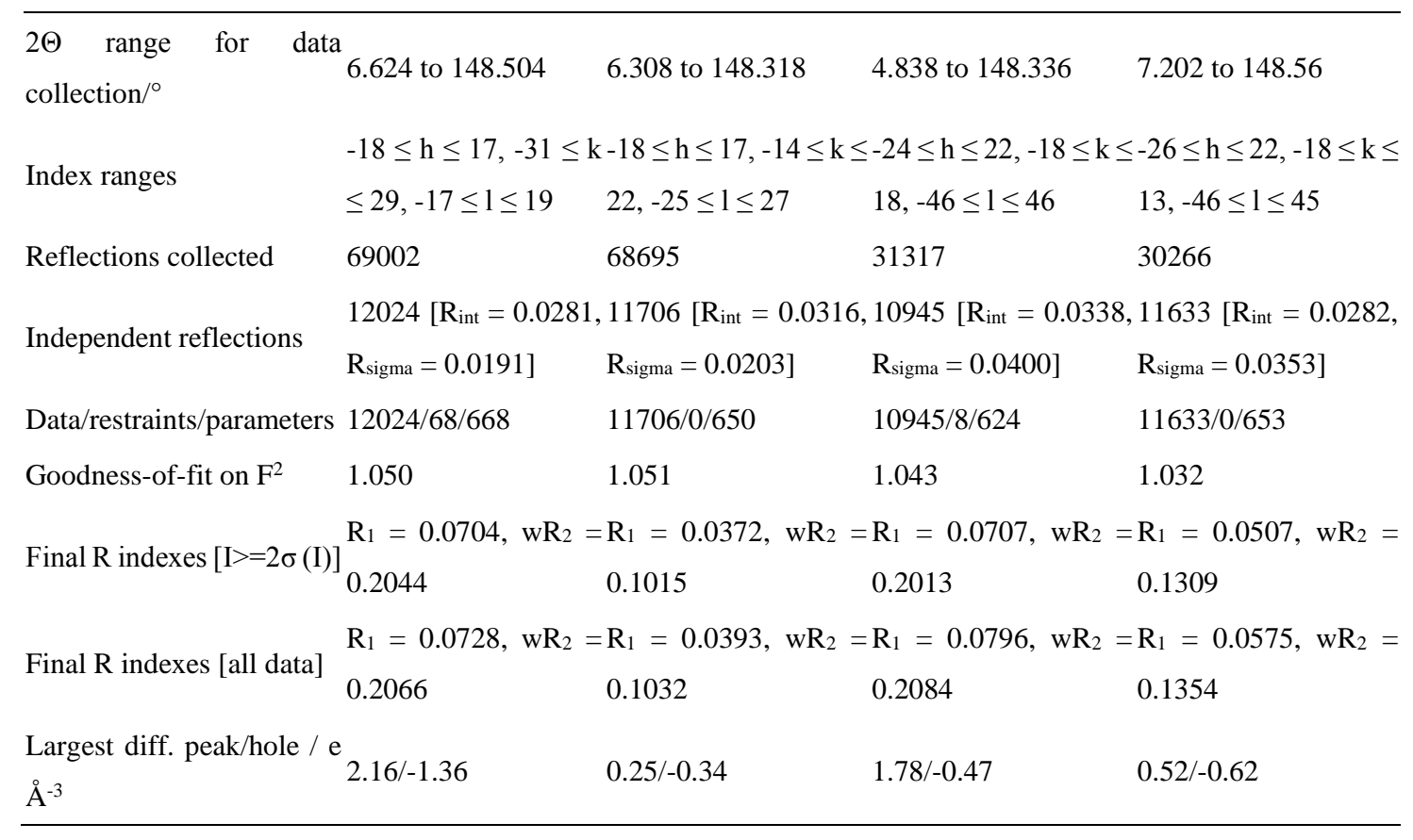

to be continued:

\begin{tabular}{|c|c|c|c|}
\hline Compounds & $\mathbf{3 a}^{\mathbf{a}^{+}}\left(\mathrm{BAr}^{\mathrm{F}}\right)$ & $\mathbf{3 b}^{\mathbf{*}}\left(\mathrm{BAr}^{\mathrm{F}}\right)$ & $3 c^{\bullet+}\left(\mathrm{BAr}^{\mathrm{F}}\right)$ \\
\hline $\mathrm{CCDC}$ & 2051359 & 2051360 & 2051361 \\
\hline Empirical formula & $\mathrm{C}_{90} \mathrm{H}_{94} \mathrm{BF}_{24} \mathrm{~N}_{4} \mathrm{P}_{2}$ & $\mathrm{C}_{91} \mathrm{H}_{96} \mathrm{BF}_{24} \mathrm{~N}_{4} \mathrm{P}_{2}$ & $\mathrm{C}_{93} \mathrm{H}_{100} \mathrm{BF}_{24} \mathrm{~N}_{4} \mathrm{P}_{2}$ \\
\hline Formula weight & 1760.44 & 1774.46 & 1802.51 \\
\hline Temperature/K & $160(15)$ & $103(4)$ & $104(7)$ \\
\hline Crystal system & monoclinic & monoclinic & triclinic \\
\hline Space group & $\mathrm{P} 2{ }_{1} / \mathrm{c}$ & $\mathrm{P} 21 / \mathrm{n}$ & $\mathrm{P}-1$ \\
\hline $\mathrm{a} / \AA$ & $40.5165(6)$ & $12.37270(10)$ & $12.70280(10)$ \\
\hline $\mathrm{b} / \AA$ & $20.7884(3)$ & $34.6870(6)$ & $16.90750(10)$ \\
\hline $\mathrm{c} / \AA$ & $21.6625(3)$ & $20.9493(2)$ & $21.52760(10)$ \\
\hline$\alpha /{ }^{\circ}$ & 90 & 90 & $89.3430(10)$ \\
\hline$\beta /{ }^{\circ}$ & $102.6460(10)$ & $98.6090(10)$ & $78.2090(10)$ \\
\hline$\gamma /{ }^{\circ}$ & 90 & 90 & $86.9100(10)$ \\
\hline Volume $/ \AA^{3}$ & $17803.1(4)$ & $8889.55(19)$ & $4519.40(5)$ \\
\hline Z & 8 & 4 & 2 \\
\hline$\rho_{\text {calc }} \mathrm{g} / \mathrm{cm}^{3}$ & 1.314 & 1.326 & 1.325 \\
\hline$\mu / \mathrm{mm}^{-1}$ & 1.285 & 1.291 & 1.278 \\
\hline$F(000)$ & 7304.0 & 3684.0 & 1874.0 \\
\hline Crystal size $/ \mathrm{mm}^{3}$ & $0.2 \times 0.18 \times 0.15$ & $0.12 \times 0.08 \times 0.08$ & $0.06 \times 0.05 \times 0.02$ \\
\hline Radiation & $\mathrm{CuK} \alpha(\lambda=1.54184)$ & $\mathrm{CuK} \alpha(\lambda=1.54184)$ & $\mathrm{CuK} \alpha(\lambda=1.54184)$ \\
\hline $2 \Theta$ range for data collection $/{ }^{\circ}$ & 4.802 to 148.636 & 5.096 to 148.314 & 5.234 to 148.242 \\
\hline
\end{tabular}




\begin{tabular}{|c|c|c|c|}
\hline \multirow{2}{*}{ Index ranges } & \multicolumn{3}{|c|}{$-43 \leq \mathrm{h} \leq 49,-25 \leq \mathrm{k} \leq 22,-15 \leq \mathrm{h} \leq 14,-42 \leq \mathrm{k} \leq 31,-15 \leq \mathrm{h} \leq 15,-18 \leq \mathrm{k} \leq 20$} \\
\hline & $-25 \leq 1 \leq 26$ & $-24 \leq 1 \leq 15$ & $-26 \leq 1 \leq 26$ \\
\hline Reflections collected & 109390 & 52319 & 53765 \\
\hline Independent reflections & $\begin{array}{l}35172\left[R_{\text {int }}=\right. \\
\left.R_{\text {sigma }}=0.0606\right]\end{array}$ & $\begin{array}{l}17454 \quad\left[R_{\text {int }}=\right. \\
\left.R_{\text {sigma }}=0.0391\right]\end{array}$ & $\begin{array}{l}17786\left[R_{\text {int }}=0.0298,\right. \\
\left.R_{\text {sigma }}=0.0302\right]\end{array}$ \\
\hline Data/restraints/parameters & $35172 / 786 / 2782$ & $17454 / 0 / 1171$ & $17786 / 11 / 1136$ \\
\hline Goodness-of-fit on $\mathrm{F}^{2}$ & 1.022 & 1.041 & 1.078 \\
\hline Final $\mathrm{R}$ indexes $[\mathrm{I}>=2 \sigma(\mathrm{I})]$ & $\mathrm{R}_{1}=0.0894, \mathrm{wR}_{2}$ & $\mathrm{R}_{1}=0.0673, w \mathrm{R}_{2}$ & $2 \mathrm{R}_{1}=0.0455, w \mathrm{R}_{2}=0.1206$ \\
\hline Final $\mathrm{R}$ indexes [all data] & $\mathrm{R}_{1}=0.1357, \mathrm{wR}_{2}$ & $\mathrm{R}_{1}=0.0744, \mathrm{wR}_{2}$ & $\mathrm{R}_{1}=0.0529, \mathrm{wR}_{2}=0.1248$ \\
\hline Largest diff. peak/hole / e $\AA^{-3}$ & $0.75 /-0.69$ & $1.14 /-0.64$ & $1.28 /-0.70$ \\
\hline
\end{tabular}

\section{S3: CV and EPR analysis}

Cyclic voltammetry was performed on an Metrohm Autolab PGSTAT302N electrochemical workstation with platinum as the working and couter electrodes, $\mathrm{Ag} / \mathrm{Ag}^{+}$as the reference electrode and $0.1 \mathrm{M} n-\mathrm{Bu}_{4}(\mathrm{BARF})$ as the supporting electrolyte.
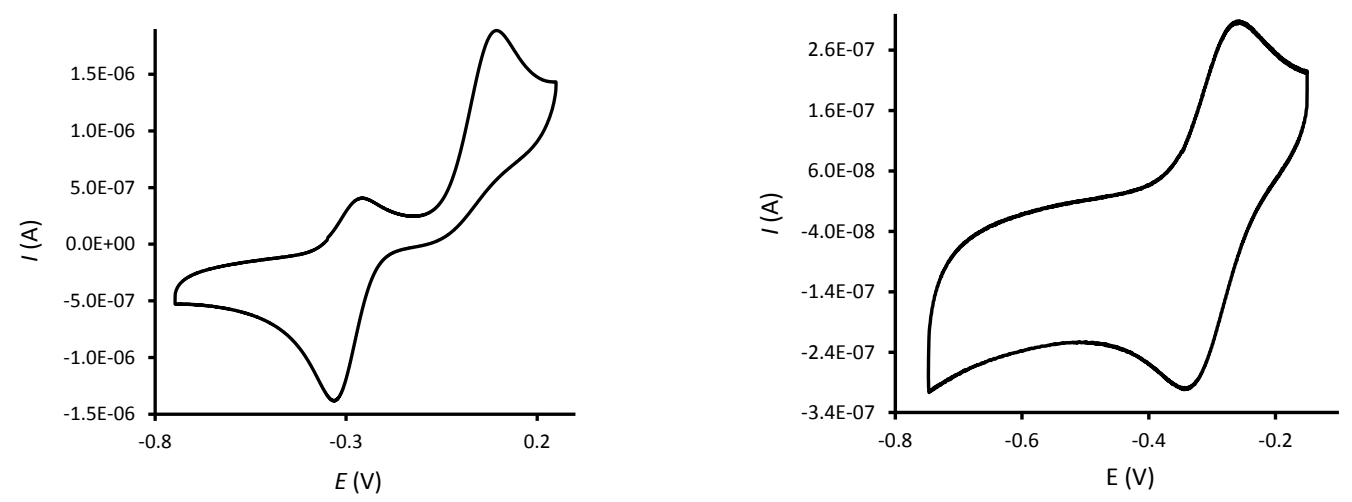

Figure S22. Cyclic voltammogram for $10^{-3}$ M THF solution of 3a containing $0.1 \mathrm{M} n$ $\mathrm{Bu}_{4} \mathrm{~N}(\mathrm{BARF})$ as electrolyte (potential versus $\mathrm{Fc}^{+} / \mathrm{Fc}$ ) at scan rate $100 \mathrm{mVs}^{-1}$ in different scan potentials.

Table S2. Spin Hamiltonian parameters and spin populations from CW EPR experiments at RT and $100 \mathrm{~K}$, and from Density Functional Theory (DFT) calculations ${ }^{[2]}$ (using ORCA 4.2.1 ${ }^{[3]}$ ). Given are the g-matrix, and ${ }^{31} \mathrm{P}$ and ${ }^{1} \mathrm{H}$ hyperfine interactions (in 
MHz). DFT parameters given in parentheses were calculated with addition of the ZORA scalar relativistic Hamiltonian using the QZVPP basis set for P and TZVP basis set for all other nuclei at B3LYP level.

\begin{tabular}{|c|c|c|c|c|c|c|c|c|}
\hline \multirow{2}{*}{ Interaction } & \multicolumn{3}{|c|}{ Principal Values } & \multirow{2}{*}{$\begin{array}{l}\text { Isotropic } \\
\text { Values }\end{array}$} & \multirow{2}{*}{$\begin{array}{l}\text { Spin } \\
\text { population }^{(a, b)}\end{array}$} & \multirow{2}{*}{\multicolumn{3}{|c|}{ Rotation Matrix R }} \\
\hline & 1 & 2 & 3 & & & & & \\
\hline \multicolumn{9}{|l|}{$3 \mathbf{a}^{++}$} \\
\hline g-matrix & $\begin{array}{l}2.0034 \\
(1.9982)\end{array}$ & $\begin{array}{l}2.0042 \\
(2.0029)\end{array}$ & $\begin{array}{l}2.0148 \\
(2.0058)\end{array}$ & $\begin{array}{l}2.0075 \\
(2.0023)\end{array}$ & n.a. & $\begin{array}{l}0.1008 \\
0.4582 \\
0.8830\end{array}$ & $\begin{array}{l}0.2116 \\
0.8574 \\
-0.4691\end{array}$ & $\begin{array}{l}-0.9721 \\
0.2341 \\
-0.0105\end{array}$ \\
\hline $31 P$ & $\begin{array}{l}242.5 \\
(181.2)\end{array}$ & $\begin{array}{l}252.9 \\
(185.8)\end{array}$ & $\begin{array}{l}353.0 \\
(413.3)\end{array}$ & $\begin{array}{l}282.8 \\
(260.1)\end{array}$ & $\begin{array}{l}0.074 \\
(0.16)\end{array}$ & $\begin{array}{l}0.2053 \\
0.5956 \\
0.7766 \\
\end{array}$ & $\begin{array}{l}0.9584 \\
-0.2829 \\
-0.0364\end{array}$ & $\begin{array}{c}0.1981 \\
0.7518 \\
-0.6290\end{array}$ \\
\hline $31 P$ & $\begin{array}{l}148.8 \\
(237.3)\end{array}$ & $\begin{array}{l}170.5 \\
(242.8)\end{array}$ & $\begin{array}{l}529.1 \\
(514.2)\end{array}$ & $\begin{array}{l}282.8 \\
(331.4)\end{array}$ & $\begin{array}{l}0.251 \\
(0.21)\end{array}$ & $\begin{array}{l}0.1009 \\
0.6719 \\
0.7337\end{array}$ & $\begin{array}{l}0.9863 \\
-0.1642 \\
0.0147\end{array}$ & $\begin{array}{r}0.1304 \\
0.7222 \\
-0.6793\end{array}$ \\
\hline \multicolumn{9}{|c|}{ 年 } \\
\hline g-matrix & $\begin{array}{l}2.0060 \\
(1.9979)\end{array}$ & $\begin{array}{l}2.0070 \\
(2.0030)\end{array}$ & $\begin{array}{l}2.0104 \\
(2.0062)\end{array}$ & $\begin{array}{l}2.0078 \\
(2.0024)\end{array}$ & n.a. & $\begin{array}{l}-0.0401 \\
0.5598 \\
0.8276 \\
\end{array}$ & $\begin{array}{r}-0.0373 \\
0.8268 \\
-0.5611 \\
\end{array}$ & $\begin{array}{c}-0.9984 \\
-0.0534 \\
-0.0122\end{array}$ \\
\hline $31 P$ & $\begin{array}{l}175.2 \\
(199.22)\end{array}$ & $\begin{array}{l}186.8 \\
(204.43)\end{array}$ & $\begin{array}{l}471.3 \\
(459.66)\end{array}$ & $\begin{array}{l}279.4 \\
(287.77)\end{array}$ & $\begin{array}{l}0.20 \\
(0.19)\end{array}$ & $\begin{array}{l}-0.0237 \\
0.7308 \\
0.6822 \\
\end{array}$ & $\begin{array}{c}0.9991 \\
0.0408 \\
-0.0090 \\
\end{array}$ & $\begin{array}{c}-0.0344 \\
0.6813 \\
-0.7311\end{array}$ \\
\hline $31 P$ & $\begin{array}{l}210.1 \\
(164.04)\end{array}$ & $\begin{array}{l}220.6 \\
(168.47)\end{array}$ & $\begin{array}{l}402.6 \\
(414.88)\end{array}$ & $\begin{array}{l}279.4 \\
(249.13)\end{array}$ & $\begin{array}{l}0.134 \\
(0.19)\end{array}$ & $\begin{array}{l}-0.0557 \\
0.7334 \\
0.6776\end{array}$ & $\begin{array}{l}0.9983 \\
0.0311 \\
0.0483\end{array}$ & $\begin{array}{r}0.0143 \\
0.6791 \\
-0.7339\end{array}$ \\
\hline \multicolumn{9}{|c|}{ 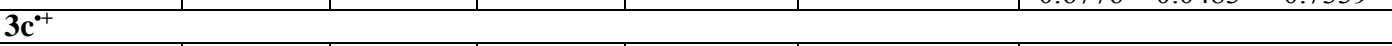 } \\
\hline g-matrix & $\begin{array}{l}2.0067 \\
(1.9983)\end{array}$ & $\begin{array}{l}2.0085 \\
(2.0030)\end{array}$ & $\begin{array}{l}2.0084 \\
(2.0058)\end{array}$ & $\begin{array}{l}2.0078 \\
(2.0024)\end{array}$ & n.a. & $\begin{array}{l}-0.2080 \\
0.4648 \\
0.8606\end{array}$ & $\begin{array}{l}-0.2618 \\
0.8213 \\
-0.5069\end{array}$ & $\begin{array}{c}-0.9424 \\
-0.3308 \\
-0.0491\end{array}$ \\
\hline $31 P$ & $\begin{array}{l}170.0 \\
(179.9)\end{array}$ & $\begin{array}{l}180.1 \\
(187.1)\end{array}$ & $\begin{array}{l}402.8 \\
(403.3)\end{array}$ & $\begin{array}{l}251.0 \\
(256.8)\end{array}$ & $\begin{array}{l}0.16 \\
(0.17)\end{array}$ & $\begin{array}{l}-0.3050 \\
0.5278 \\
0.7927 \\
\end{array}$ & $\begin{array}{l}0.9215 \\
0.3736 \\
0.1059 \\
\end{array}$ & $\begin{array}{c}-0.2402 \\
0.7628 \\
-0.6003 \\
\end{array}$ \\
\hline $31 P$ & $\begin{array}{l}212.9 \\
(207.9)\end{array}$ & $\begin{array}{l}222.6 \\
(210.6)\end{array}$ & $\begin{array}{l}470.5 \\
(489.3)\end{array}$ & $\begin{array}{l}302.3 \\
(302.6)\end{array}$ & $\begin{array}{l}0.18 \\
(0.21)\end{array}$ & $\begin{array}{l}-0.1487 \\
0.7498 \\
0.6448\end{array}$ & $\begin{array}{c}0.9820 \\
0.1887 \\
0.0071 \\
\end{array}$ & $\begin{array}{c}-0.1163 \\
0.6342 \\
-0.7643 \\
\end{array}$ \\
\hline
\end{tabular}

(a) Spin populations, given is the total, then contributions from s-type and p-type orbitals.

$2.0059 \quad-0.00008 \quad-0.0000101$

$-0.0000005 \quad 2.0015340 \quad 0.0008430$

$0.0001338 \quad 0.0035721 \quad 1.9997854$

(b) Experimental spin populations for s-type and p-type contributions were calculated using $a_{\text {iso }} / S O, T / P O$, where $a$ iso is the isotropic part of the hyperfine interaction and $T$ the dipolar constant. This is calculated as $\left(A_{1}, A_{2}, A_{3}\right)=a_{\text {iso }}+\left(-T_{1},-T_{2}, T_{1}+T_{2}\right), T=\left(T_{1}+T_{2}\right) / 2$.

\section{S4: Theoretical Details}

Calculations were carried out with the Gaussian 16 package. ${ }^{[2]}$ Geometry optimizations were performed with the B3PW91 functional ${ }^{[3]}$ augmented with the D3BJ version of Grimme's empirical dispersion correction. ${ }^{[4]}$ The Def2-SVP basis set was used for all the atoms. Frequency calculations at the same level of theory were performed to identify the number of imaginary frequencies (zero for local minimum and one for transition states) and provide the thermal corrections of Gibbs free energy. Transition 
states were submitted to intrinsic reaction coordinate (IRC) calculations to determine two corresponding minima. The single-point energy calculations were performed at the B3PW91/Def2-TZVP level of theory for solution-phase (tetrahydrofuran). The gasphase geometry was used for all the solution phase calculations. The SMD method was used with the corresponding solvent, while Bondi radii ${ }^{[5]}$ were chosen as the atomic radii to define the molecular cavity. The Gibbs energy and enthalpy corrections from frequency calculations were added to the single-point energies to obtain the Gibbs free energies and enthalpies in solution, respectively. All the solution-phase free energies reported in the paper correspond to the reference state of $1 \mathrm{~mol} / \mathrm{L}, 298 \mathrm{~K}$. We also compared free energies for species cis-3a, trans-3a, cis-3a ${ }^{\cdot+}$ and trans $\mathbf{- 3 a}{ }^{\cdot+}$ with 4 commonly used functionals (PBE ${ }^{[6]}, \mathrm{B} 3 \mathrm{LYP}+\mathrm{D} 3 \mathrm{BJ} \mathrm{J}^{[7]}, \mathrm{M} 06-2 \mathrm{X}^{[8]}$ ) (Table S8)

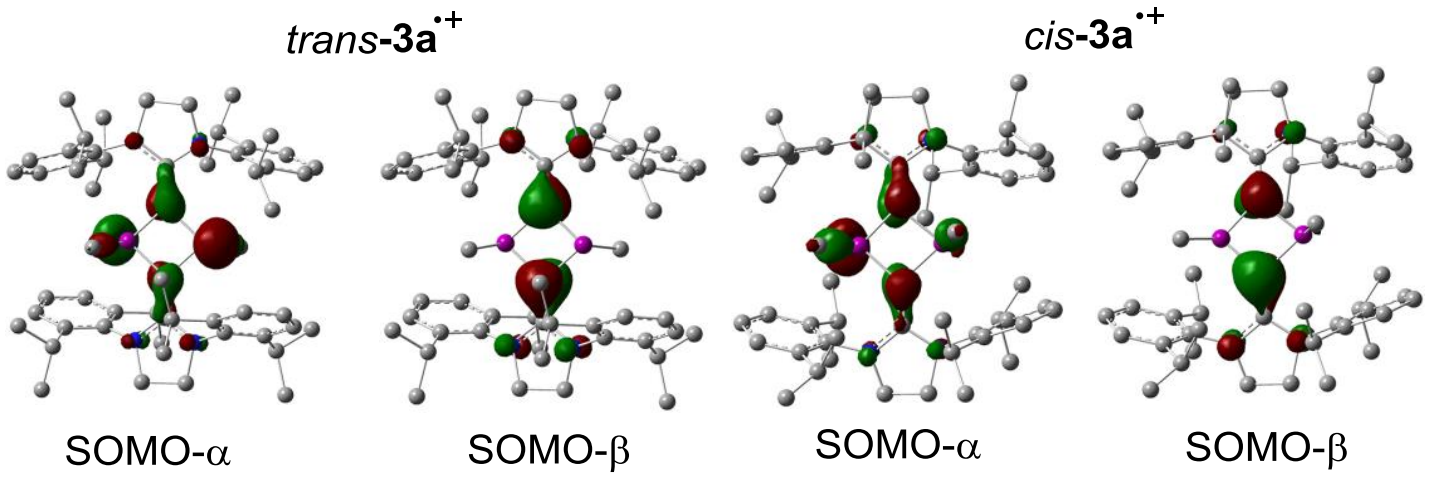

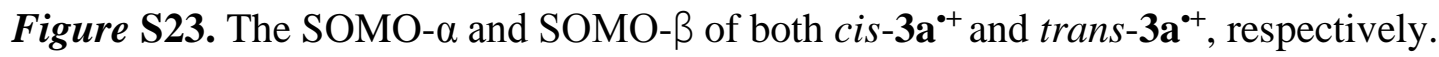

Table S3. Energies of Intermediates and Transition States using B3PW91+D3BJ

\begin{tabular}{lll}
\hline Species & $\begin{array}{l}\text { Thermal Corrections of Gibbs } \\
\text { Free Energies (Hartree) }\end{array}$ & Solvation energies (Hartree) \\
\hline cis-3a & 1.180377 & -3161.610539 \\
TS1 & 1.179299 & -3161.554106 \\
trans-3a & 1.180071 & -3161.609398 \\
& 1.144479 & -3121.571471 \\
& & \\
Me- II & & \\
& & \\
& & \\
TS2 & 1.322774 & -5213.300989 \\
TS3 & 1.321649 & -5213.296651 \\
\hline
\end{tabular}




\begin{tabular}{lll}
\hline $\mathrm{Zn}(\mathrm{Me})_{2}(\mathrm{thf})$ & 0.147452 & -2091.731779 \\
$\mathrm{Zn}(\mathrm{Me})(\mathrm{thf})(\mathrm{I})$ & 0.111775 & -2349.746303 \\
$\mathrm{I}^{-}$ & -0.01685 & -298.023233 \\
cis-3a++ $^{\cdot+}$ & 1.181542 & -3161.463191 \\
$\mathbf{T S 4}$ & 1.182879 & -3161.451732 \\
${\text { trans }-\mathbf{3 a}^{\cdot+}}$ & 1.181342 & -3161.478411 \\
\hline
\end{tabular}

Table $\mathbf{S 4}$. Energies of Intermediates and Transition States using PBE

\begin{tabular}{lll}
\hline Species & $\begin{array}{l}\text { Thermal Corrections of Gibbs } \\
\text { Free Energies (Hartree) }\end{array}$ & Solvation energies (Hartree) \\
\hline cis-3a & 1.133262 & -3158.533183 \\
trans $-\mathbf{3 a}$ & 1.132837 & -3158.531262 \\
${\text { cis }-3 a^{++}}_{\text {trans }-\mathbf{3 a}}{ }^{++}$ & 1.132548 & -3158.39138 \\
\hline
\end{tabular}

Table S5. Energies of Intermediates and Transition States using B3LYP+D3BJ

\begin{tabular}{lll}
\hline Species & $\begin{array}{l}\text { Thermal Corrections of Gibbs } \\
\text { Free Energies (Hartree) }\end{array}$ & Solvation energies (Hartree) \\
\hline cis-3a & 1.177258 & -3162.630576 \\
trans-3a & 1.176061 & -3162.627503 \\
${\text { cis }-3 a^{\bullet+}}_{\text {trans }^{-3} \mathbf{a}^{\cdot+}}$ & 1.17654 & -3162.482584 \\
\hline
\end{tabular}

Table S6. Energies of Intermediates and Transition States using M06-2X

\begin{tabular}{lll}
\hline Species & $\begin{array}{l}\text { Thermal Corrections of Gibbs Free } \\
\text { Energies (Hartree) }\end{array}$ & Solvation energies (Hartree) \\
\hline cis-3a & 1.190532 & -3161.107034 \\
trans $-\mathbf{3 a}$ & 1.189052 & -3161.10225 \\
cis $\mathbf{3} \mathbf{a}^{\bullet+}$ & 1.18831 & -3160.946196 \\
trans $^{-3} \mathbf{3 a}$ & 1.187 & -3160.957353 \\
\hline
\end{tabular}

Table $S 7$. Comparison of Geometric Parameters $(\AA)$.

\begin{tabular}{|l|l|l|l|l|l|}
\hline & P-C & C-L & P-R & $\angle \mathrm{NCN}$ & $\angle \mathrm{CPC}$ \\
\hline $\begin{array}{l}\text { Crystallographic } \\
\text { data }\end{array}$ & $1.827(1)$ & $1.354(2)$ & $1.852(2)$ & $106.52(9)$ & $98.09(5)$ \\
\hline PBE & 1.851 & 1.375 & 1.879 & 106.41 & 97.64 \\
\hline B3LYP-D3BJ & 1.848 & 1.360 & 1.869 & 106.50 & 98.11 \\
\hline M062X & 1.848 & 1.360 & 1.869 & 106.04 & 97.55 \\
\hline B3PW91-D3BJ & 1.837 & 1.359 & 1.858 & 106.75 & 98.25 \\
\hline
\end{tabular}


Table S8. Comparison of Free Energies (kcal/mol)

\begin{tabular}{|l|l|l|l|l|}
\hline & cis-3a & trans-3a & cis-3a $^{\bullet+}$ & trans-3a $^{++}$ \\
\hline PBE & 0 & 0.9 & 0 & -7.6 \\
\hline B3LYP+D3BJ & 0 & 1.2 & 0 & -7.7 \\
\hline M062X & 0 & 2.1 & 0 & -7.8 \\
\hline B3PW91+D3BJ & 0 & 0.5 & 0 & -9.7 \\
\hline
\end{tabular}

\section{Cartesian Coordinates}

\section{cis-3a}

P $\quad-0.13276000$

P $\quad-0.01146600$

N 3.14768500

N -3.21181400

N 3.39005600

N -3.30466100

C -2.50250400

C 2.45875600

C 3.12408800

C 2.45880100

C 2.85053400

C 2.52260300

C $\quad-2.92635700$

C 1.12450000

C 4.48316800

H 5.20656300

H $\quad 4.50979800$

C 3.53085900

C 2.98920600

C -2.22467400

C -1.26519700

C 4.74084700

H $\quad 5.29543100$

H 5.31013900

C -3.09806300

C 2.35482900

H 2.30381400

C -3.53963800

C 2.07271600

H 1.82671600

C -2.59969700

C $\quad-3.35033600$

C 2.39744000

H 1.95373700

C $\quad-2.20134400$
$-1.37072100$

1.40478600

$-1.37783200$

1.33764800

0.80577700

$-0.87861200$

0.14972100

$-0.16720700$

2.15534300

$-3.65966300$

$-2.38436600$

2.45082800

$-2.24323700$

$-0.03309100$

$-1.23610900$

$-1.83982500$

$-1.56363200$

3.18167500

$-2.10997100$

$-2.68135400$

0.06504100

0.26354000

0.67545300

0.49936300

2.44015500

$-3.95109400$

$-2.97142600$

2.31048400

1.34506300

0.47250200

3.66560400

$-3.15116200$

3.79178000

4.04334300

3.82249800
$-0.21585600$

$-0.13984300$

$-0.12902300$

0.73821100

0.26708200

1.01833400

0.53620100

$-0.06550600$

0.61128300

$-0.63042500$

$-1.08448200$

1.85427500

1.15697500

$-0.28779700$

0.43218200

$-0.13284600$

1.48769600

$-0.26661100$

$-2.46275900$

2.29636400

$-0.01979500$

0.30975600

1.16557300

$-0.60766700$

$-0.15877300$

0.85081300

1.35104700

$-1.49349300$

2.79071500

2.16905600

0.32698600

0.16203300

2.22213400

3.18584700

1.77795800
H $\quad-2.06470000$

C 2.19751500

2.80276300

2.16974500

H 1.88629300

$-4.65421700$

$-1.57861700$

C -1.62571300

$-5.64595800$

$-1.24417200$

H -1.77488300

$-1.71330600$

3.29879400

C -4.51733000

$-0.69456000$

2.91006500

H $\quad-5.30989700$

1.06596400

1.32315700

H $\quad-4.77617900$

1.09029600

0.55157800

C -2.51969600

1.80435500

2.09598300

H -2.12601200

4.75000200

$-0.55290700$

C 3.45866400

5.70313400

$-0.19466000$

H 3.52871900

$-0.76078400$

$-2.96683700$

C -2.04415900

$-0.09341300$

$-2.10055500$

H $\quad-1.51670500$

$-4.05671400$

2.47322800

C 2.71123200

$-4.41881000$

3.35847500

H 2.80701100

$-3.13146600$

$-3.37556900$

C -3.43888000

$-2.93468600$

$-4.44617200$

H $\quad-3.76412700$

3.42027900

$-2.33836200$

C 2.81768500

3.33332200

$-3.37789600$

H 2.70341400

4.81596300

1.37757300

C 3.36700600

5.85812900

1.68560100

H 3.68008700

4.51131600

0.13819900

C -2.49076900

5.31882500

$-0.52761800$

H -2.32815000

$-4.96686300$

1.52278500

C 4.17242900

$-6.03696800$

1.67253000

H 3.99433400

2.87205700

$-1.60547300$

C -4.12610200

1.80480400

$-1.80200700$

H $\quad-4.14905200$

1.02133200

$-2.03456100$

C -2.92965100

0.29039000

$-1.21614400$

H $\quad-2.85354500$

4.63036200

$-1.87727100$

C -0.43745400

5.48550200

$-2.55345400$

H -0.37420600

$-1.85936200$

$-1.98211600$

H $\quad-1.44617500$

$-1.01061300$

$-2.67524400$

H $\quad 0.28408800$

$-2.28983300$

$-2.03560300$

C 2.31751000

$-2.63119800$

$-2.28133900$

H $\quad 2.10229600 \quad-5.18062500 \quad-3.66878900$ 


\begin{tabular}{|c|c|c|c|c|c|c|c|}
\hline $\mathrm{C}$ & -3.11364100 & -4.51370600 & 0.36269000 & $\mathrm{H}$ & -6.20127900 & 1.63211400 & -1.70695200 \\
\hline $\mathrm{H}$ & -3.42838400 & -5.23510600 & -0.39268800 & $\mathrm{H}$ & -6.00393400 & 0.26363100 & -2.83204400 \\
\hline C & 0.80122500 & 1.70543900 & 3.55485300 & $\mathrm{H}$ & -5.62159700 & 1.91559900 & -3.36133600 \\
\hline $\mathrm{H}$ & 0.94991200 & 2.54941600 & 4.24647700 & $\mathrm{C}$ & -2.30532400 & -1.81717800 & 4.66661600 \\
\hline $\mathrm{H}$ & 0.46596400 & 0.85102500 & 4.15929700 & $\mathrm{H}$ & -3.39383300 & -1.67021800 & 4.59521600 \\
\hline $\mathrm{H}$ & -0.00240200 & 1.96332700 & 2.85080600 & $\mathrm{H}$ & -1.90240300 & -1.06264000 & 5.36058600 \\
\hline C & -0.05688000 & 2.04556500 & -1.88148400 & $\mathrm{H}$ & -2.13735900 & -2.80841700 & 5.11730800 \\
\hline $\mathrm{H}$ & 0.92360600 & 2.50605800 & -2.06951600 & $\mathrm{C}$ & 4.85823800 & -0.85684400 & -3.57875900 \\
\hline $\mathrm{H}$ & -0.82894400 & 2.82160200 & -1.97458000 & $\mathrm{H}$ & 5.57698200 & -1.29026800 & -2.86620400 \\
\hline $\mathrm{H}$ & -0.23062800 & 1.25907400 & -2.62708800 & $\mathrm{H}$ & 5.22409900 & 0.14100600 & -3.86949300 \\
\hline $\mathrm{C}$ & -0.11380000 & -1.92636700 & 3.41353000 & $\mathrm{H}$ & 4.86199500 & -1.49125100 & -4.47943900 \\
\hline $\mathrm{H}$ & 0.12378900 & -2.92861000 & 3.80171100 & $\mathrm{C}$ & 2.45740200 & -0.12021900 & -3.92538000 \\
\hline $\mathrm{H}$ & 0.32881200 & -1.19331400 & 4.10319500 & $\mathrm{H}$ & 2.33251400 & -0.71212300 & -4.84584700 \\
\hline $\mathrm{H}$ & 0.36325600 & -1.81899200 & 2.42839100 & $\mathrm{H}$ & 2.79272000 & 0.88666400 & -4.21819900 \\
\hline $\mathrm{C}$ & -4.05103600 & -2.64687600 & -1.08586800 & $\mathrm{H}$ & 1.47692900 & -0.02224200 & -3.44185300 \\
\hline $\mathrm{H}$ & -3.57245100 & -1.688688300 & -1.33846500 & $\mathrm{C}$ & -3.24760100 & 0.42652100 & -3.13426600 \\
\hline $\mathrm{C}$ & -4.33426500 & -0.33268100 & 1.88964400 & $\mathrm{H}$ & -3.15540500 & 1.10975100 & -3.99317700 \\
\hline $\mathrm{H}$ & -3.99672100 & -0.29202600 & 2.94170500 & $\mathrm{H}$ & -3.67187800 & -0.52017300 & -3.50302300 \\
\hline $\mathrm{H}$ & -5.24982100 & -0.94013500 & 1.85413000 & $\mathrm{H}$ & -2.24276200 & 0.22107700 & -2.74556200 \\
\hline $\mathrm{C}$ & 3.20803300 & 0.94445100 & 3.73658200 & $\mathrm{C}$ & -3.32558600 & 4.49455900 & 2.57360300 \\
\hline $\mathrm{H}$ & 4.10708100 & 0.64149400 & 3.17946000 & $\mathrm{H}$ & -3.49559300 & 5.52428400 & 2.21917800 \\
\hline $\mathrm{H}$ & 2.90531300 & 0.09893300 & 4.37462500 & $\mathrm{H}$ & -3.07145700 & 4.54387600 & 3.64452900 \\
\hline $\mathrm{H}$ & 3.49076000 & 1.78350800 & 4.39275300 & $\mathrm{H}$ & -4.27798300 & 3.95264500 & 2.47347000 \\
\hline $\mathrm{C}$ & 3.60861700 & -4.67995100 & 1.34540100 & $\mathrm{C}$ & -3.89753500 & -3.57478000 & -2.28876700 \\
\hline $\mathrm{H}$ & 3.69919800 & -5.67063100 & 0.87074500 & $\mathrm{H}$ & -2.84677200 & -3.84586300 & -2.46858200 \\
\hline $\mathrm{H}$ & 3.56913900 & -4.83094100 & 2.43607000 & $\mathrm{H}$ & -4.27955800 & -3.08162300 & -3.19559900 \\
\hline $\mathrm{H}$ & 4.52583700 & -4.11875300 & 1.11192000 & $\mathrm{H}$ & -4.47023100 & -4.50737500 & -2.16300600 \\
\hline $\mathrm{C}$ & 1.08389500 & -4.71372700 & 1.20638100 & $\mathrm{C}$ & -5.53363600 & -2.36617100 & -0.82511200 \\
\hline $\mathrm{H}$ & 0.19940900 & -4.16261800 & 0.85908400 & $\mathrm{H}$ & -6.05427900 & -3.28417000 & -0.50909700 \\
\hline $\mathrm{H}$ & 1.00273000 & -4.83316600 & 2.29735400 & $\mathrm{H}$ & -6.02481200 & -1.99363300 & -1.73742900 \\
\hline $\mathrm{H}$ & 1.06673200 & -5.72390200 & 0.76689200 & $\mathrm{H}$ & -5.67130000 & -1.61125600 & -0.04086800 \\
\hline $\mathrm{C}$ & -0.88269800 & 4.56965500 & 1.93986800 & & & & \\
\hline $\mathrm{H}$ & -0.09155600 & 4.07855100 & 1.35697000 & \multicolumn{4}{|c|}{ TS1 } \\
\hline $\mathrm{H}$ & -0.57621300 & 4.57856100 & 2.99670800 & $\mathrm{P}$ & 0.12737900 & -1.32831500 & 0.05302900 \\
\hline $\mathrm{H}$ & -0.95859000 & 5.61980800 & 1.61551900 & $\mathrm{P}$ & -0.04720300 & 1.26364600 & 0.39090200 \\
\hline $\mathrm{C}$ & 5.68564300 & 3.11222700 & -1.55475700 & $\mathrm{~N}$ & -3.38876900 & -1.24172400 & 0.68459400 \\
\hline $\mathrm{H}$ & 5.90281900 & 4.18302300 & -1.41192300 & $\mathrm{~N}$ & 3.18110500 & 1.49935200 & -0.58865700 \\
\hline $\mathrm{H}$ & 6.16735900 & 2.79309500 & -2.49275700 & $\mathrm{~N}$ & -3.30968800 & 1.01056700 & 0.58655000 \\
\hline $\mathrm{H}$ & 6.15954100 & 2.56988400 & -0.72407400 & $\mathrm{~N}$ & 3.38859000 & -0.65335400 & -1.12814500 \\
\hline $\mathrm{C}$ & 3.54943300 & 3.65459000 & -2.76199900 & $\mathrm{C}$ & 2.51285200 & 0.27409500 & -0.55511500 \\
\hline $\mathrm{H}$ & 2.46245400 & 3.50329000 & -2.81342800 & $\mathrm{C}$ & -2.52844600 & -0.15400100 & 0.52068100 \\
\hline $\mathrm{H}$ & 3.98381200 & 3.32962200 & -3.72028800 & $\mathrm{C}$ & -3.19772000 & 2.10152600 & -0.31602700 \\
\hline $\mathrm{H}$ & 3.73360200 & 4.73639400 & -2.67066900 & $\mathrm{C}$ & -3.23247600 & -3.58788000 & 0.08035600 \\
\hline $\mathrm{C}$ & -5.56775000 & 1.22124500 & -2.50815600 & $\mathrm{C}$ & -2.96677300 & -2.55091700 & 0.99873000 \\
\hline
\end{tabular}




\begin{tabular}{|c|c|c|c|c|c|c|c|}
\hline $\mathrm{C}$ & -3.23585200 & 1.92081600 & -1.71750400 & $\mathrm{C}$ & -3.20082700 & 4.50537500 & -0.61426100 \\
\hline $\mathrm{C}$ & 3.27950400 & -2.06138600 & -1.02357600 & $\mathrm{H}$ & -3.16795400 & 5.51202700 & -0.19013100 \\
\hline $\mathrm{C}$ & -1.18812000 & -0.18700900 & 0.35284200 & $\mathrm{C}$ & 3.22124000 & -4.84315800 & -0.91421300 \\
\hline $\mathrm{C}$ & -4.77505000 & -0.80733400 & 0.74380700 & $\mathrm{H}$ & 3.19672100 & -5.93484600 & -0.87095600 \\
\hline $\mathrm{H}$ & -5.33364500 & -1.38832600 & 1.49236900 & $\mathrm{C}$ & -2.98347400 & 3.60030100 & 1.73129000 \\
\hline $\mathrm{H}$ & -5.29596000 & -0.92002400 & -0.22724100 & $\mathrm{H}$ & -2.81862000 & 2.59988100 & 2.15997900 \\
\hline $\mathrm{C}$ & -3.13844100 & 3.39869900 & 0.23818900 & $\mathrm{C}$ & 4.23599200 & 1.92815500 & 2.05339800 \\
\hline $\mathrm{C}$ & -2.27720300 & -2.80382400 & 2.20909100 & $\mathrm{H}$ & 4.28962400 & 1.05097200 & 1.39418200 \\
\hline $\mathrm{C}$ & 3.00819200 & -2.79465300 & -2.19967900 & $\mathrm{C}$ & 2.01166900 & 4.96910000 & 1.46661900 \\
\hline $\mathrm{C}$ & 1.26548700 & 0.04267700 & -0.07312600 & $\mathrm{H}$ & 1.69598800 & 5.86389500 & 2.00885500 \\
\hline $\mathrm{C}$ & -4.62405000 & 0.66816900 & 1.12104500 & $\mathrm{C}$ & 0.24486700 & -3.13797700 & -0.06859900 \\
\hline $\mathrm{H}$ & -5.39637800 & 1.30695300 & 0.67310300 & $\mathrm{H}$ & 0.69678100 & -3.61048500 & 0.81377000 \\
\hline $\mathrm{H}$ & -4.65726200 & 0.79884000 & 2.21842700 & $\mathrm{H}$ & 0.80124500 & -3.42752400 & -0.96937200 \\
\hline $\mathrm{C}$ & 2.78562700 & 2.67354000 & 0.09508100 & $\mathrm{H}$ & -0.78618600 & -3.49666900 & -0.16757400 \\
\hline $\mathrm{C}$ & -3.79924900 & -3.28280400 & -1.29321700 & $\mathrm{C}$ & -2.16540700 & -5.15509100 & 1.59511000 \\
\hline $\mathrm{H}$ & -4.20662300 & -2.26379900 & -1.26290500 & $\mathrm{H}$ & -1.85042000 & -6.17462600 & 1.83056600 \\
\hline $\mathrm{C}$ & 3.27225500 & 2.89697700 & 1.40045800 & $\mathrm{C}$ & 3.47785200 & -4.10772500 & 0.23732200 \\
\hline $\mathrm{C}$ & -3.18782600 & 0.55363500 & -2.37055500 & $\mathrm{H}$ & 3.65247500 & -4.62801600 & 1.18235600 \\
\hline $\mathrm{H}$ & -3.26237800 & -0.20468100 & -1.58246700 & $\mathrm{C}$ & -1.83060200 & 0.35685000 & -3.04975800 \\
\hline $\mathrm{C}$ & 1.93161000 & 3.60197100 & -0.53738100 & $\mathrm{H}$ & -1.67887900 & 1.09338700 & -3.85492500 \\
\hline $\mathrm{C}$ & 3.51920000 & -2.70883700 & 0.20526100 & $\mathrm{H}$ & -1.75209200 & -0.64807100 & -3.48912200 \\
\hline $\mathrm{C}$ & -3.28269000 & 3.05726000 & -2.53104800 & $\mathrm{H}$ & -1.01584800 & 0.47149700 & -2.32085000 \\
\hline $\mathrm{H}$ & -3.31715700 & 2.93271500 & -3.61606200 & $\mathrm{C}$ & 0.16740900 & 1.38701400 & 2.23107800 \\
\hline $\mathrm{C}$ & 1.48832400 & 3.38470300 & -1.96908600 & $\mathrm{H}$ & -0.80029900 & 1.66632400 & 2.67278400 \\
\hline $\mathrm{H}$ & 1.57499400 & 2.30383100 & -2.16356000 & $\mathrm{H}$ & 0.90076200 & 2.17086500 & 2.46513300 \\
\hline $\mathrm{C}$ & -2.84170700 & -4.89117600 & 0.40730300 & $\mathrm{H}$ & 0.49130100 & 0.42009300 & 2.64204100 \\
\hline $\mathrm{H}$ & -3.03655700 & -5.70466700 & -0.29535500 & $\mathrm{C}$ & 1.16833400 & -1.78615300 & -3.54288800 \\
\hline $\mathrm{C}$ & 2.67419600 & -2.06384300 & -3.48417100 & $\mathrm{H}$ & 0.60076400 & -2.72914300 & -3.60492900 \\
\hline $\mathrm{H}$ & 3.17511200 & -1.08541100 & -3.42886200 & $\mathrm{H}$ & 0.91403900 & -1.17827000 & -4.42542900 \\
\hline $\mathrm{C}$ & 4.36903400 & 1.41788600 & -1.41289300 & $\mathrm{H}$ & 0.82481400 & -1.25179300 & -2.64369600 \\
\hline $\mathrm{H}$ & 5.16823200 & 2.06012700 & -1.01602400 & $\mathrm{C}$ & 3.82448000 & -1.93108400 & 1.46892800 \\
\hline $\mathrm{H}$ & 4.17166800 & 1.72181700 & -2.45956200 & $\mathrm{H}$ & 3.78035400 & -0.86429900 & 1.21467000 \\
\hline $\mathrm{C}$ & 1.55169400 & 4.74533700 & 0.17125000 & $\mathrm{C}$ & 4.70092000 & -0.06658400 & -1.33680200 \\
\hline $\mathrm{H}$ & 0.87556600 & 452500 & -0.29431500 & $\mathrm{H}$ & 900 & -0.45 & 1100 \\
\hline $\mathrm{C}$ & -2.06333900 & -1.69690700 & 3.22158500 & $\mathrm{H}$ & 5.38746100 & -0.28891000 & -0.49525600 \\
\hline $\mathrm{H}$ & -1.89613100 & -0.77226600 & 2.65084200 & $\mathrm{C}$ & -4.35322800 & 0.31815100 & -3.33190900 \\
\hline $\mathrm{C}$ & 2.98393600 & -4.18981700 & -2.12346700 & $\mathrm{H}$ & -5.32195400 & 0.45790700 & -2.82727300 \\
\hline $\mathrm{H}$ & 2.77067400 & -4.77648900 & -3.01899600 & $\mathrm{H}$ & -4.32014500 & -0.70913100 & -3.72780100 \\
\hline $\mathrm{C}$ & -1.87351800 & -4.11584100 & 2.47532300 & $\mathrm{H}$ & -4.32549100 & 1.00272500 & -4.19436500 \\
\hline $\mathrm{H}$ & -1.33275200 & -4.33339400 & 3.39772800 & $\mathrm{C}$ & -4.93195200 & -4.22273100 & -1.70077500 \\
\hline $\mathrm{C}$ & 2.87167100 & 4.05719800 & 2.07199200 & $\mathrm{H}$ & -4.58129700 & -5.25950500 & -1.82525200 \\
\hline $\mathrm{H}$ & 3.23488600 & 4.24669000 & 3.08507900 & $\mathrm{H}$ & -5.36498200 & -3.90852700 & -2.66320900 \\
\hline $\mathrm{C}$ & -3.28995800 & 4.34029900 & -1.99161500 & $\mathrm{H}$ & -5.73636800 & -4.22964400 & -0.94940500 \\
\hline $\mathrm{H}$ & -3.33975800 & 5.21150700 & -2.64921700 & $\mathrm{C}$ & -2.66615300 & -3.26996600 & -2.32447800 \\
\hline
\end{tabular}




\begin{tabular}{|c|c|c|c|c|c|c|c|}
\hline $\mathrm{H}$ & -1.88870700 & -2.54308700 & -2.04491800 & $\mathrm{H}$ & 5.33494500 & -3.28005600 & 2.30140500 \\
\hline $\mathrm{H}$ & -3.04661800 & -2.99622300 & -3.32119600 & $\mathrm{H}$ & 5.46880200 & -1.59653400 & 2.85509500 \\
\hline $\mathrm{H}$ & -2.18990000 & -4.26041300 & -2.40405600 & $\mathrm{H}$ & 5.99374700 & -2.04205800 & 1.21078800 \\
\hline $\mathrm{C}$ & 0.03855600 & 3.78644100 & -2.21556700 & & & & \\
\hline $\mathrm{H}$ & -0.62859500 & 3.30086000 & -1.49148500 & \multicolumn{4}{|c|}{$\operatorname{trans} \mathbf{- 3 a}$} \\
\hline $\mathrm{H}$ & -0.27649300 & 3.48330300 & -3.22510800 & $\mathrm{P}$ & 0.02968200 & -1.33998500 & 0.06809100 \\
\hline $\mathrm{H}$ & -0.10748800 & 4.87588700 & -2.14485800 & $\mathrm{P}$ & 0.04814800 & 1.38594900 & 0.41747100 \\
\hline $\mathrm{C}$ & -4.25021400 & 4.18094000 & 2.36390000 & $\mathrm{~N}$ & 3.26639600 & -1.23889900 & -0.37880500 \\
\hline $\mathrm{H}$ & -4.47226600 & 5.18152800 & 1.95854400 & $\mathrm{~N}$ & -3.32268700 & 1.13217200 & 0.70435300 \\
\hline $\mathrm{H}$ & -4.13619200 & 4.27951100 & 3.45537000 & $\mathrm{~N}$ & 3.51785000 & 0.94992000 & 0.02614700 \\
\hline $\mathrm{H}$ & -5.12642200 & 3.54410000 & 2.16941900 & $\mathrm{~N}$ & -3.32290900 & -1.09418500 & 0.79460200 \\
\hline 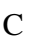 & -1.75234700 & 4.45246300 & 2.04613900 & $\mathrm{C}$ & -2.51085000 & 0.01117200 & 0.55533100 \\
\hline $\mathrm{H}$ & -0.85807800 & 4.05095000 & 1.54895900 & $\mathrm{C}$ & 2.56771900 & -0.05544300 & -0.11284600 \\
\hline $\mathrm{H}$ & -1.56103200 & 4.47733100 & 3.13068000 & $\mathrm{C}$ & 3.23028700 & 2.26239500 & 0.47011500 \\
\hline $\mathrm{H}$ & -1.88255400 & 5.49374900 & 1.70986500 & $\mathrm{C}$ & 2.72681700 & -3.58111700 & -0.78598900 \\
\hline $\mathrm{C}$ & 5.64028100 & 2.53293400 & 2.13806400 & $\mathrm{C}$ & 2.79444700 & -2.25139700 & -1.25491100 \\
\hline 11 & 5.99867300 & 2.85740900 & 1.14933700 & $\mathrm{C}$ & 2.76167700 & 2.45902000 & 1.78955100 \\
\hline $\mathrm{H}$ & 6.35840400 & 1.79997700 & 2.53900600 & $\mathrm{C}$ & -2.88329400 & -2.43715400 & 0.89017700 \\
\hline $\mathrm{H}$ & 5.65494600 & 3.41488000 & 2.79856700 & $\mathrm{C}$ & 1.22159900 & 0.04500400 & 0.00391200 \\
\hline $\mathrm{C}$ & 3.16565500 & -2.77735500 & -4.74063800 & $\mathrm{C}$ & 4.69060200 & -1.04306400 & -0.14463100 \\
\hline $\mathrm{H}$ & 4.24002900 & -3.01119300 & -4.68205100 & $\mathrm{H}$ & 5.28374800 & -1.60244000 & -0.88071600 \\
\hline $\mathrm{H}$ & 3.00173000 & -2.14371000 & -5.62586400 & $\mathrm{H}$ & 4.98287100 & -1.38288900 & 0.86614600 \\
\hline $\mathrm{H}$ & 2.62467000 & -3.72072400 & -4.91790500 & $\mathrm{C}$ & 3.39692600 & 3.34811200 & -0.41757800 \\
\hline $\mathrm{C}$ & -3.32349400 & -1.50827200 & 4.07245100 & $\mathrm{C}$ & 2.43230900 & -1.93846800 & -2.58376300 \\
\hline $\mathrm{H}$ & -4.21129600 & -1.33345200 & 3.44848600 & $\mathrm{C}$ & -2.87089600 & -3.06262400 & 2.15669900 \\
\hline $\mathrm{H}$ & -3.21197800 & -0.64726700 & 4.75074500 & $\mathrm{C}$ & -1.18332900 & 0.02921200 & 0.25897800 \\
\hline $\mathrm{H}$ & -3.52113500 & -2.40238300 & 4.68568600 & $\mathrm{C}$ & 4.85448500 & 0.46919900 & -0.28522000 \\
\hline$c$ & -0.83350600 & -1.89755600 & 4.09977300 & $\mathrm{H}$ & 5.59205000 & 0.88601900 & 0.41634100 \\
\hline 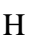 & -0.94731300 & -2.74182900 & 4.79898600 & $\mathrm{H}$ & 5.16949900 & 0.74021800 & -1.30946600 \\
\hline $\mathrm{H}$ & -0.65309100 & -0.99731900 & 4.70667100 & $\mathrm{C}$ & -3.13041400 & 2.35872200 & 0.02295800 \\
\hline $\mathrm{H}$ & 0.05970400 & -2.07354800 & 3.48479300 & $\mathrm{C}$ & 3.10268200 & -3.93231400 & 0.63789000 \\
\hline $\mathrm{C}$ & 3.75480500 & 1.44299200 & 3.41986700 & $\mathrm{H}$ & 3.11546700 & -2.98352200 & 1.19694500 \\
\hline $\mathrm{H}$ & 3.68785800 & 2.26816200 & 4.14671500 & $\mathrm{C}$ & -3.36854000 & 2.42603300 & -1.36638500 \\
\hline $\mathrm{H}$ & 4.45551400 & 0.69958900 & 3.83076900 & $\mathrm{C}$ & 2.67479000 & 9800 & 2.76261000 \\
\hline $\mathrm{H}$ & 2.76476500 & 0.97410700 & 3.34635200 & $\mathrm{H}$ & 2.39227900 & 0.40973400 & 2.18134500 \\
\hline$C$ & 2.43299100 & 4.12288600 & -2.92565700 & $\mathrm{C}$ & -2.75271900 & 3.50018100 & 0.75872000 \\
\hline 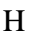 & 2.35743900 & 5.21253100 & -2.77903900 & $\mathrm{C}$ & -2.45105700 & -3.12069100 & -0.26989300 \\
\hline $\mathrm{H}$ & 2.17535600 & 3.90441200 & -3.97428900 & $\mathrm{C}$ & 2.46479400 & 3.76339200 & 2.19620600 \\
\hline $\mathrm{H}$ & 3.48435500 & 3.84241000 & -2.76518400 & $\mathrm{H}$ & 2.09351700 & 3.93739200 & 3.20679100 \\
\hline $\mathrm{C}$ & 2.76122700 & -2.16792700 & 2.54127900 & $\mathrm{C}$ & -2.49479700 & 3.41063700 & 2.24841900 \\
\hline $\mathbf{H}$ & 1.77043700 & -1.87610300 & 2.15911300 & $\mathrm{H}$ & -2.37123700 & 2.33986000 & 2.47405100 \\
\hline 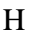 & 2.97292300 & -1.56719400 & 3.43844200 & $\mathrm{C}$ & 2.30912500 & -4.58631700 & -1.66382000 \\
\hline $\mathrm{H}$ & 2.72006100 & -3.22507200 & 2.84920000 & $\mathrm{H}$ & 2.25462900 & -5.61807200 & -1.30994200 \\
\hline 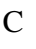 & 5.23387800 & -2.22961100 & 1.98495900 & $\mathrm{C}$ & -3.32129200 & -2.33557100 & 3.40993700 \\
\hline
\end{tabular}




\begin{tabular}{|c|c|c|c|c|c|c|c|}
\hline $\mathrm{H}$ & -3.49128900 & -1.28552100 & 3.13016200 & $\mathrm{H}$ & -2.60003600 & -1.75544800 & 5.37627400 \\
\hline $\mathrm{C}$ & -4.61959800 & 0.77150200 & 1.25368500 & $\mathrm{H}$ & -1.31792700 & -1.87945100 & 4.14614600 \\
\hline $\mathrm{H}$ & -5.41726100 & 1.36884000 & 0.78957400 & $\mathrm{C}$ & -2.60015000 & -2.49058400 & -1.64208600 \\
\hline $\mathrm{H}$ & -4.65690300 & 0.94149300 & 2.34650900 & $\mathrm{H}$ & -2.41864800 & -1.41320900 & -1.52176200 \\
\hline $\mathrm{C}$ & -2.63511400 & 4.71888600 & 0.08210900 & $\mathrm{C}$ & -4.72016000 & -0.71393400 & 0.92140000 \\
\hline $\mathrm{H}$ & -2.34138400 & 5.61422500 & 0.63397500 & $\mathrm{H}$ & -5.22171500 & -1.29355400 & 1.70796500 \\
\hline 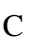 & 2.49135300 & -0.52928600 & -3.13819700 & $\mathrm{H}$ & -5.27373500 & -0.88288700 & -0.02160900 \\
\hline $\mathrm{H}$ & 2.77494600 & 0.14599000 & -2.32278300 & $\mathrm{C}$ & 4.05012900 & 1.03391200 & 3.38386100 \\
\hline $\mathrm{C}$ & -2.41825400 & -4.38252500 & 2.24396400 & $\mathrm{H}$ & 4.81196100 & 0.85373900 & 2.61108600 \\
\hline 11 & -2.39906900 & -4.88218200 & 3.21548100 & $\mathrm{H}$ & 4.01873100 & 0.15037000 & 4.04132900 \\
\hline 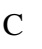 & 2.00585500 & -2.97355600 & -3.42035200 & $\mathrm{H}$ & 4.38344000 & 1.89502500 & 3.98536700 \\
\hline $\mathrm{H}$ & 1.71600700 & -2.74289000 & -4.44824200 & $\mathrm{C}$ & 4.50872100 & -4.53751400 & 0.70619300 \\
\hline $\mathrm{C}$ & -3.22760600 & 3.66233900 & -2.00400900 & $\mathrm{H}$ & 4.55056800 & -5.48751200 & 0.14924900 \\
\hline $\mathrm{H}$ & -3.39972400 & 3.73448900 & -3.08081100 & $\mathrm{H}$ & 4.79839700 & -4.74449000 & 1.74889800 \\
\hline 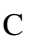 & 2.63732200 & 4.84371600 & 1.33599500 & $\mathrm{H}$ & 5.26311600 & -3.86713300 & 0.26937800 \\
\hline $\mathrm{H}$ & 2.39880800 & 5.85451500 & 1.67580900 & $\mathrm{C}$ & 2.07638800 & -4.85803200 & 1.29458200 \\
\hline $\mathrm{C}$ & 3.10711200 & 4.63636800 & 0.04276000 & $\mathrm{H}$ & 1.05189300 & -4.48874000 & 1.14861800 \\
\hline $\mathrm{H}$ & 3.23062500 & 5.48825800 & -0.62957900 & $\mathrm{H}$ & 2.26413600 & -4.93654700 & 2.37679500 \\
\hline $\mathrm{C}$ & -1.95333900 & -5.05372400 & 1.11602600 & $\mathrm{H}$ & 2.12894200 & -5.87767300 & 0.88082500 \\
\hline $\mathrm{H}$ & -1.57484300 & -6.07492100 & 1.20581400 & $\mathrm{C}$ & -1.20481800 & 4.11796200 & 2.65901900 \\
\hline $\mathrm{C}$ & 3.88642700 & 3.13548700 & -1.83853500 & $\mathrm{H}$ & -0.35453600 & 3.72758900 & 2.08071400 \\
\hline $\mathrm{H}$ & 3.76205200 & 2.06461800 & -2.05824000 & $\mathrm{H}$ & -0.99916400 & 3.94555500 & 3.72707800 \\
\hline $\mathrm{C}$ & -3.76998200 & 1.20453400 & -2.16874200 & $\mathrm{H}$ & -1.26401200 & 5.20784200 & 2.50919700 \\
\hline $\mathrm{H}$ & -3.85196800 & 0.36211300 & -1.46996900 & $\mathrm{C}$ & 5.37398300 & 3.48773000 & -1.95464800 \\
\hline $\mathrm{C}$ & -2.87122200 & 4.80193700 & -1.28758200 & $\mathrm{H}$ & 5.52865000 & 4.56479400 & -1.78106800 \\
\hline $\mathrm{H}$ & -2.76725400 & 5.76023600 & -1.80248400 & $\mathrm{H}$ & 5.76030000 & 3.24850200 & -2.95826700 \\
\hline $\mathrm{C}$ & 0.32278800 & -1.85934800 & 1.83809400 & $\mathrm{H}$ & 5.98207700 & 2.94797500 & -1.21448200 \\
\hline $\mathrm{H}$ & -0.31434000 & -2.71097300 & 2.10761700 & $\mathrm{C}$ & 3.07438600 & 3.89891900 & -2.88591400 \\
\hline $\mathrm{H}$ & 0.13290000 & -1.01641400 & 2.51821600 & $\mathrm{H}$ & 2.00421000 & 3.65853100 & -2.82254800 \\
\hline $\mathrm{H}$ & 1.37507100 & -2.16044700 & 1.93218500 & $\mathrm{H}$ & 3.42229800 & 3.63636700 & -3.89723500 \\
\hline $\mathrm{C}$ & 1.94833300 & -4.28950300 & -2.97286300 & $\mathrm{H}$ & 3.18424500 & 4.98926100 & -2.77761100 \\
\hline $\mathrm{H}$ & 1.61419400 & -5.08341700 & -3.64518400 & $\mathrm{C}$ & -5.14018400 & 1.38585400 & -2.82456800 \\
\hline $\mathrm{C}$ & -1.96721100 & -4.42585400 & -0.12545900 & $\mathrm{H}$ & -5.90927700 & 1.64159000 & -2.07939500 \\
\hline $\mathrm{H}$ & -1.59491200 & -4.95805200 & -1.00140500 & $\mathrm{H}$ & -5.45046500 & 0.45902100 & -3.33212200 \\
\hline $\mathrm{C}$ & 1.60135300 & 1.47146500 & 3.83240400 & $\mathrm{H}$ & -5.12779500 & 2.18965100 & -3.57771200 \\
\hline $\mathrm{H}$ & 1.84242300 & 2.27719100 & 4.54471700 & $\mathrm{C}$ & -4.63789500 & -2.91710600 & 3.93572500 \\
\hline $\mathrm{H}$ & 1.50097000 & 0.54360100 & 4.41623500 & $\mathrm{H}$ & -5.41857300 & -2.92917600 & 3.15969100 \\
\hline $\mathrm{H}$ & 0.63037800 & 1.69165800 & 3.36494400 & $\mathrm{H}$ & -5.01275700 & -2.33346100 & 4.79148100 \\
\hline$C$ & 0.03544400 & 2.42795500 & -1.10973600 & $\mathrm{H}$ & -4.50060100 & -3.95668300 & 4.27376500 \\
\hline $\mathrm{H}$ & 1.00486100 & 2.94313500 & -1.11590900 & $\mathrm{C}$ & 3.55509100 & -0.39548000 & -4.22951900 \\
\hline $\mathrm{H}$ & -0.74999900 & 3.18985000 & -1.04979500 & $\mathrm{H}$ & 4.54784200 & -0.69035700 & -3.85525800 \\
\hline $\mathrm{H}$ & -0.07571500 & 1.84303900 & -2.02967200 & $\mathrm{H}$ & 3.61857800 & 0.64646900 & -4.58227500 \\
\hline $\mathrm{C}$ & -2.25211900 & -2.33179000 & 4.50454800 & $\mathrm{H}$ & 3.32715800 & -1.03030300 & -5.10047800 \\
\hline $\mathrm{H}$ & -2.02219300 & -3.35078100 & 4.85362400 & $\mathrm{C}$ & 1.11156400 & -0.08002600 & -3.61571400 \\
\hline
\end{tabular}




$\begin{array}{rrrr}\mathrm{H} & 0.76963400 & -0.66457200 & -4.48433000 \\ \mathrm{H} & 1.12480300 & 0.98080700 & -3.90927700 \\ \mathrm{H} & 0.37596600 & -0.20824200 & -2.80945700 \\ \mathrm{C} & -2.69337700 & 0.82790800 & -3.18620300 \\ \mathrm{H} & -2.50868500 & 1.64292400 & -3.90371400 \\ \mathrm{H} & -2.99362300 & -0.06286700 & -3.75862700 \\ \mathrm{H} & -1.74916000 & 0.60036200 & -2.67465600 \\ \mathrm{C} & -3.70075500 & 3.93137800 & 3.03689600 \\ \mathrm{H} & -3.86203500 & 5.00467700 & 2.84466200 \\ \mathrm{H} & -3.54681600 & 3.80250700 & 4.12017500 \\ \mathrm{H} & -4.62587000 & 3.40465700 & 2.75799200 \\ \mathrm{C} & -1.59646100 & -3.00369100 & -2.66851700 \\ \mathrm{H} & -0.57194600 & -2.93127000 & -2.27896900 \\ \mathrm{H} & -1.65647300 & -2.39914300 & -3.58629500 \\ \mathrm{H} & -1.79423700 & -4.04915000 & -2.95638000 \\ \mathrm{C} & -4.03639000 & -2.67605700 & -2.14522300 \\ \mathrm{H} & -4.26471600 & -3.74460600 & -2.28749500 \\ \mathrm{H} & -4.17841300 & -2.16774600 & -3.11195100 \\ \mathrm{H} & -4.77421800 & -2.27180300 & -1.43836200\end{array}$<smiles></smiles>

P -0.17963800

P $\quad-0.02170900$

N 3.22142400

N $\quad-3.32863500$

N 3.24010100

$\mathrm{N} \quad-3.50217300$

C -2.63127200

C 2.45237400

C 2.82680400

C 3.07662600

C 2.74247300

C 2.71806300

C -3.16460500

C 1.07799400

C 4.55976800

H 4.63084800

H 5.32826300

C 2.51039900

$-1.29874800$

1.33148900

$-0.93124300$

1.32626300

1.15920100

$-0.86812100$

0.16759900

0.07202000

2.30593200

$-3.34246000$

$-2.19293900$

2.21795600

$-0.27982400$

0.23534100

$-0.93361300$

0.50927700

$-0.23389300$

0.50949000

0.45291300

$-0.44045000$

0.49885500

$-0.64084500$

$-1.38745400$

1.90238900

$-2.21652500$

0.81715000

0.02561500

$-0.16962500$

$-0.44305600$

$-1.26770900$

$-0.26296800$

$-2.35408900$

$-1.17498200$

$-0.99127400$

$-0.21009800$
C 1.98312400

$-2.26001700$

$-2.57357400$

C -3.06919600

$-2.58002900$

2.17636800

C -1.23418700

0.06317000

0.34219200

C 4.64666900

0.84999900

$-0.46311500$

H 5.16804200

0.71125300

0.50061600

H 5.14526600

1.66402500

$-1.00686000$

C $\quad-2.76137400$

2.61808900

0.34470600

C 4.00567700

$-3.26925100$

0.55775700

H 4.07775700

$-2.20855900$

0.84712400

C -2.64507400

3.13724800

$-0.95943800$

C 3.06749300

0.95018600

2.65877400

H 3.29325000

0.16687700

1.91989300

C -2.30123200

3.31250900

1.47892900

C -2.98303600

$-3.14329800$

$-0.22755300$

C 2.27590900

3.35129500

2.58949100

H 2.18182200

3.31876600

3.67699800

C -2.44827700

2.73405500

2.87114400

H $\quad-2.72801200$

1.67569800

2.75578000

C 2.58182700

$-4.57289600$

$-1.08351300$

H 2.81780400

$-5.48081500$

$-0.52481700$

C -3.23554300

$-1.55731400$

3.28495700

H -3.77591500

$-0.69569600$

2.86361300

C -4.75163500

1.09982600

0.73809300

H $\quad-5.35243400$

1.71197700

0.05068600

H $\quad-5.02560500$

1.37959200

1.76992100

C -1.70167300

4.55871400

1.27850000

H -1.32944300

5.12118000

2.13651100

C 1.75499400

$-1.04115600$

$-3.44795600$

H 1.97782200

$-0.14633200$

$-2.84810200$

C $\quad-2.76901400$

$-3.91265600$

2.47445200

H -2.68136000

$-4.22693200$

3.51593700

C 1.51410600

$-3.51498800$

$-2.97438600$

H 0.92326500

$-3.60027600$

$-3.88794400$

C -2.03383400

4.38514200

$-1.10878600$

H -1.92157900

4.81416200

$-2.10662400$

C 1.95456800

4.52234600

1.90687400

H 1.60996000

5.39672100

2.46376000

C 2.07533800

4.58665900

0.52282800

H 1.81933400

5.51031900

0.00010800

C -2.57090700

$-4.84394500$

1.45723900

H $\quad-2.33262200$

$-5.87987700$

1.70918600

C 2.62365300

3.54212200

$-1.71887300$

H 2.86629600

2.52554900

$-2.06563500$

C -3.13835900

2.36671200

$-2.16786900$ 


\begin{tabular}{|c|c|c|c|c|c|c|c|}
\hline $\mathrm{H}$ & -3.66485700 & 1.47431800 & -1.79688200 & $\mathrm{C}$ & 1.29548900 & 3.93577900 & -2.36467300 \\
\hline $\mathrm{C}$ & -1.56305000 & 5.08587400 & -0.00192400 & $\mathrm{H}$ & 0.48270500 & 3.28426800 & -2.01559900 \\
\hline $\mathrm{H}$ & -1.08553200 & 6.05867300 & -0.13918600 & $\mathrm{H}$ & 1.36048400 & 3.86113800 & -3.46110800 \\
\hline $\mathrm{C}$ & 0.23662400 & -2.39089600 & 1.14127800 & $\mathrm{H}$ & 1.01756500 & 4.97233400 & -2.11877400 \\
\hline $\mathrm{H}$ & -0.63501300 & -2.99882800 & 1.40718600 & $\mathrm{C}$ & -4.13780900 & 3.18122300 & -2.99136200 \\
\hline $\mathrm{H}$ & 0.58237700 & -1.82544100 & 2.01555700 & $\mathrm{H}$ & -4.98072000 & 3.52735600 & -2.37427300 \\
\hline $\mathrm{H}$ & 1.02660900 & -3.06673600 & 0.79278300 & $\mathrm{H}$ & -4.54247000 & 2.57536700 & -3.81648900 \\
\hline $\mathrm{C}$ & 1.79696300 & -4.65669500 & -2.23073000 & $\mathrm{H}$ & -3.66566300 & 4.07005800 & -3.43758500 \\
\hline $\mathrm{H}$ & 1.41629300 & -5.62662700 & -2.55895600 & $\mathrm{C}$ & -4.06752100 & -2.08100800 & 4.45505000 \\
\hline $\mathrm{C}$ & -2.67504200 & -4.46284900 & 0.12266600 & $\mathrm{H}$ & -5.03293100 & -2.48600400 & 4.11627500 \\
\hline $\mathrm{H}$ & -2.52024100 & -5.20483200 & -0.66202000 & $\mathrm{H}$ & -4.26818300 & -1.27025200 & 5.17156200 \\
\hline $\mathrm{C}$ & 1.89365900 & 0.43888800 & 3.49488400 & $\mathrm{H}$ & -3.54434700 & -2.87640800 & 5.00743100 \\
\hline $\mathrm{H}$ & 1.64038000 & 1.13371000 & 4.30982200 & $\mathrm{C}$ & 2.72730800 & -1.06401700 & -4.63368000 \\
\hline $\mathrm{H}$ & 2.14109600 & -0.53101900 & 3.95286200 & $\mathrm{H}$ & 3.77419000 & -1.14109100 & -4.30351500 \\
\hline $\mathrm{H}$ & 0.99741000 & 0.30423000 & 2.87347800 & $\mathrm{H}$ & 2.62401700 & -0.15057900 & -5.23971700 \\
\hline $\mathrm{C}$ & -1.87104900 & -1.03970400 & 3.75209400 & $\mathrm{H}$ & 2.52741100 & -1.92721600 & -5.28751000 \\
\hline $\mathrm{H}$ & -1.25933900 & -1.86034100 & 4.15807400 & $\mathrm{C}$ & 0.31029800 & -0.90475000 & -3.92315200 \\
\hline $\mathrm{H}$ & -1.99317800 & -0.28467300 & 4.54337200 & $\mathrm{H}$ & 0.02865400 & -1.71448100 & -4.61346800 \\
\hline $\mathrm{H}$ & -1.31579300 & -0.58092200 & 2.92218300 & $\mathrm{H}$ & 0.17682500 & 0.04483300 & -4.46193400 \\
\hline $\mathrm{C}$ & -3.17929300 & -2.74770600 & -1.67740200 & $\mathrm{H}$ & -0.38362900 & -0.92482700 & -3.07153700 \\
\hline $\mathrm{H}$ & -3.03547200 & -1.65658900 & -1.73651100 & $\mathrm{C}$ & -1.97120300 & 1.86979100 & -3.02348500 \\
\hline $\mathrm{C}$ & -4.88959800 & -0.40214000 & 0.47336200 & $\mathrm{H}$ & -1.41737500 & 2.70998800 & -3.46943100 \\
\hline $\mathrm{H}$ & -5.48728600 & -0.92222200 & 1.23478700 & $\mathrm{H}$ & -2.33476500 & 1.23387000 & -3.84501200 \\
\hline $\mathrm{H}$ & -5.33616800 & -0.61150700 & -0.51222700 & $\mathrm{H}$ & -1.26448900 & 1.27919800 & -2.42360000 \\
\hline $\mathrm{C}$ & 4.32118100 & 1.15221900 & 3.51524100 & $\mathrm{C}$ & -3.57210600 & 3.43809300 & 3.63760200 \\
\hline $\mathrm{H}$ & 5.17308600 & 1.49726500 & 2.90990200 & $\mathrm{H}$ & -3.33311300 & 4.50126200 & 3.79716800 \\
\hline $\mathrm{H}$ & 4.60965000 & 0.21263100 & 4.01131400 & $\mathrm{H}$ & -3.72147000 & 2.97523200 & 4.62540600 \\
\hline $\mathrm{H}$ & 4.14969500 & 1.90536300 & 4.29989800 & $\mathrm{H}$ & -4.52607900 & 3.39574800 & 3.09053400 \\
\hline $\mathrm{C}$ & 5.40504000 & -3.75729600 & 0.15831500 & $\mathrm{C}$ & -2.16223400 & -3.39658400 & -2.61285300 \\
\hline $\mathrm{H}$ & 5.38654900 & -4.83206200 & -0.08067600 & $\mathrm{H}$ & -1.13707700 & -3.20868700 & -2.26495100 \\
\hline $\mathrm{H}$ & 6.12244300 & -3.60773800 & 0.97988500 & $\mathrm{H}$ & -2.26377100 & -2.98273200 & -3.62705700 \\
\hline $\mathrm{H}$ & 5.78745400 & -3.23751200 & -0.73282100 & $\mathrm{H}$ & -2.31318900 & -4.48435800 & -2.69134100 \\
\hline $\mathrm{C}$ & 3.50753400 & -4.04080000 & 1.78056800 & $\mathrm{C}$ & -4.61231500 & -3.06831300 & -2.12080500 \\
\hline $\mathrm{H}$ & 2.52619400 & -3.68446000 & 2.12111200 & $\mathrm{H}$ & -4.78969400 & -4.15505300 & -2.10186900 \\
\hline $\mathrm{H}$ & 4.21646500 & -3.92204000 & 2.61391700 & $\mathrm{H}$ & -4.79120600 & -2.71490400 & -3.14805300 \\
\hline $\mathrm{H}$ & 3.42579400 & -5.11965400 & 1.57812700 & $\mathrm{H}$ & -5.36278800 & -2.60540400 & -1.46347500 \\
\hline $\mathrm{C}$ & -1.13264500 & 2.76693400 & 3.64560900 & & & & \\
\hline $\mathrm{H}$ & -0.32584000 & 2.29484600 & 3.06932300 & \multicolumn{2}{|c|}{ TS2 } & & \\
\hline $\mathrm{H}$ & -1.23358400 & 2.23365400 & 4.60278800 & $\mathrm{P}$ & 0.03819800 & -1.12564700 & 0.64966400 \\
\hline $\mathrm{H}$ & -0.81946900 & 3.79715400 & 3.87432300 & $\mathrm{P}$ & 0.03109200 & 1.31109400 & -0.58815300 \\
\hline $\mathrm{C}$ & 3.76259800 & 4.47103700 & -2.14744700 & $\mathrm{~N}$ & 3.31405900 & 1.07241800 & -0.78394300 \\
\hline $\mathrm{H}$ & 3.56678600 & 5.50931500 & -1.83653000 & $\mathrm{~N}$ & -3.31978500 & 1.00653500 & -0.68487500 \\
\hline $\mathrm{H}$ & 3.87909500 & 4.46676100 & -3.24219600 & $\mathrm{~N}$ & 3.29535500 & -1.13578500 & -0.55594700 \\
\hline $\mathrm{H}$ & 4.72176600 & 4.16968300 & -1.69939500 & $\mathrm{~N}$ & -3.29770300 & -0.89352500 & 0.45697300 \\
\hline
\end{tabular}




\begin{tabular}{|c|c|c|c|c|c|c|c|}
\hline $\mathrm{C}$ & -2.51016300 & 0.13427400 & 0.00023600 & $\mathrm{H}$ & 3.56262100 & 3.99124500 & 2.77062200 \\
\hline $\mathrm{C}$ & 2.53460400 & -0.01039300 & -0.44644200 & $\mathrm{C}$ & -2.36283600 & 3.61882100 & -3.15094400 \\
\hline $\mathrm{C}$ & 2.83562300 & -2.48325900 & -0.51643100 & $\mathrm{H}$ & -2.03489900 & 3.70713200 & -4.18820600 \\
\hline $\mathrm{C}$ & 2.98825100 & 3.46626900 & -1.04987300 & $\mathrm{C}$ & 2.15979700 & -5.17898100 & -0.53721800 \\
\hline $\mathrm{C}$ & 3.20424200 & 2.36199700 & -0.20004300 & $\mathrm{H}$ & 1.89942600 & -6.23969900 & -0.55090200 \\
\hline $\mathrm{C}$ & 2.10617700 & -2.99822300 & -1.60640800 & $\mathrm{C}$ & 2.84923800 & -4.64561000 & 0.54633500 \\
\hline $\mathrm{C}$ & -2.94506400 & -1.70616900 & 1.57546200 & $\mathrm{H}$ & 3.12611400 & -5.29355100 & 1.38026100 \\
\hline $\mathrm{C}$ & 1.20735800 & 0.08016900 & -0.08778300 & $\mathrm{C}$ & -2.29983200 & -3.30514300 & 3.75301400 \\
\hline $\mathrm{C}$ & 4.55201000 & 0.63180000 & -1.42695300 & $\mathrm{H}$ & -2.04161000 & -3.93311300 & 4.60897500 \\
\hline $\mathrm{H}$ & 5.39764000 & 1.25065500 & -1.09879500 & $\mathrm{C}$ & 3.99730900 & -2.74184900 & 1.74576500 \\
\hline $\mathrm{H}$ & 4.47312000 & 0.71149500 & -2.52468800 & $\mathrm{H}$ & 3.92456000 & -1.64379900 & 1.69133800 \\
\hline $\mathrm{C}$ & 3.20302900 & -3.29305000 & 0.57798200 & $\mathrm{C}$ & -2.29682200 & 1.11330800 & -3.43545700 \\
\hline $\mathrm{C}$ & 3.39049700 & 2.53096200 & 1.19197400 & $\mathrm{H}$ & -2.26734200 & 0.24703400 & -2.75522200 \\
\hline $\mathrm{C}$ & -3.02394400 & -1.16090500 & 2.87382500 & $\mathrm{C}$ & -2.62168300 & 4.76869600 & -2.41631400 \\
\hline $\mathrm{C}$ & -1.14385400 & 0.21456500 & 0.18306400 & $\mathrm{H}$ & -2.50959000 & 5.75015400 & -2.88335100 \\
\hline $\mathrm{C}$ & 4.66248900 & -0.82302800 & -0.97068100 & $\mathrm{C}$ & 0.41807100 & -0.88457100 & 2.44725800 \\
\hline $\mathrm{H}$ & 4.97465300 & -1.49889300 & -1.77868500 & $\mathrm{H}$ & -0.31693700 & -1.41762200 & 3.06479000 \\
\hline $\mathrm{H}$ & 5.36658800 & -0.94415200 & -0.13200800 & $\mathrm{H}$ & 0.45473700 & 0.17115800 & 2.74390800 \\
\hline $\mathrm{C}$ & -2.97115200 & 2.26458300 & -1.25177900 & $\mathrm{H}$ & 1.40641400 & -1.33116100 & 2.62272700 \\
\hline $\mathrm{C}$ & 2.69666100 & 3.27597700 & -2.52403700 & $\mathrm{C}$ & 3.24105100 & 4.94089200 & 0.86488600 \\
\hline $\mathrm{H}$ & 2.53349200 & 2.19720500 & -2.67344000 & $\mathrm{H}$ & 3.28550400 & 5.94993000 & 1.28070700 \\
\hline $\mathrm{C}$ & -2.53614600 & 2.34807300 & -2.58936200 & $\mathrm{C}$ & -2.23837400 & -3.82840600 & 2.46448800 \\
\hline $\mathrm{C}$ & 1.66033200 & -2.13628600 & -2.77044300 & $\mathrm{H}$ & -1.93236900 & -4.86561800 & 2.31856000 \\
\hline $\mathrm{H}$ & 1.96298300 & -1.10020000 & -2.56327500 & $\mathrm{C}$ & 0.13505400 & -2.13941100 & -2.89246800 \\
\hline $\mathrm{C}$ & -3.18050700 & 3.42620100 & -0.47017700 & $\mathrm{H}$ & -0.25008800 & -3.14800000 & -3.10613000 \\
\hline $\mathrm{C}$ & -2.56527100 & -3.04484300 & 1.35327000 & $\mathrm{H}$ & -0.18621100 & -1.48036600 & -3.71002300 \\
\hline $\mathrm{C}$ & 1.78827400 & -4.35865300 & -1.59722500 & $\mathrm{H}$ & -0.33299600 & -1.79205400 & -1.95951600 \\
\hline $\mathrm{H}$ & 1.23142500 & -4.78347100 & -2.43495400 & $\mathrm{C}$ & -2.26676100 & 1.13078200 & 3.59791800 \\
\hline $\mathrm{C}$ & -3.58745900 & 3.32729800 & 0.98945200 & $\mathrm{H}$ & -1.81767900 & 0.72707700 & 4.51825500 \\
\hline $\mathrm{H}$ & -3.08241800 & 2.43596300 & 1.39054100 & $\mathrm{H}$ & -2.59399000 & 2.15999200 & 3.81008500 \\
\hline $\mathrm{C}$ & 3.04212800 & 4.75268900 & -0.49910400 & $\mathrm{H}$ & -1.48724900 & 1.17017900 & 2.82588200 \\
\hline $\mathrm{H}$ & 2.93166600 & 5.61870200 & -1.15518900 & $\mathrm{C}$ & -2.56190000 & -3.63282500 & -0.04163400 \\
\hline $\mathrm{C}$ & -3.44417200 & 0.27456300 & 3.12607300 & $\mathrm{H}$ & -2.50748700 & -2.78338800 & -0.74038200 \\
\hline $\mathrm{H}$ & -3.77914400 & 0.69657800 & 2.16902100 & $\mathrm{C}$ & -4.69778900 & -0.68046800 & 0.10914900 \\
\hline $\mathrm{C}$ & -4.59043900 & 0.35777400 & -0.99913800 & $\mathrm{H}$ & -5.26415600 & -0.30426000 & 0.98095300 \\
\hline $\mathrm{H}$ & -4.54671000 & -0.12495200 & -1.99098800 & $\mathrm{H}$ & -5.17065000 & -1.61232000 & -0.22779500 \\
\hline $\mathrm{H}$ & -5.41529000 & 1.08186900 & -1.00889800 & $\mathrm{C}$ & 2.33739400 & -2.56324200 & -4.07500900 \\
\hline $\mathrm{C}$ & -3.01825200 & 4.67072000 & -1.08429200 & $\mathrm{H}$ & 3.43427800 & -2.53841200 & -3.98511300 \\
\hline $\mathrm{H}$ & -3.21574400 & 5.57997800 & -0.51495100 & $\mathrm{H}$ & 2.04675000 & -1.89500200 & -4.90065500 \\
\hline $\mathrm{C}$ & 3.64140800 & 1.35558000 & 2.11575300 & $\mathrm{H}$ & 2.05315000 & -3.58795400 & -4.36074500 \\
\hline $\mathrm{H}$ & 3.13922000 & 0.48143300 & 1.67895500 & $\mathrm{C}$ & 3.88519900 & 3.71059600 & -3.38603700 \\
\hline $\mathrm{C}$ & -2.69388000 & -1.98537900 & 3.95450300 & $\mathrm{H}$ & 4.07436000 & 4.79117400 & -3.28466900 \\
\hline $\mathrm{H}$ & -2.74150600 & -1.58649700 & 4.97023900 & $\mathrm{H}$ & 3.69122200 & 3.50423900 & -4.44969100 \\
\hline $\mathrm{C}$ & 3.39070300 & 3.83452400 & 1.70437100 & $\mathrm{H}$ & 4.80929100 & 3.18692500 & -3.09975500 \\
\hline
\end{tabular}




\begin{tabular}{|c|c|c|c|c|c|c|c|}
\hline $\mathrm{C}$ & 1.40824000 & 3.98852300 & -2.94007200 & $\mathrm{C}$ & -3.87133900 & -4.38603100 & -0.30381100 \\
\hline $\mathrm{H}$ & 0.55465000 & 3.65444300 & -2.33160500 & $\mathrm{H}$ & -3.96367800 & -5.25356900 & 0.36861200 \\
\hline $\mathrm{H}$ & 1.17422400 & 3.76649500 & -3.99187900 & $\mathrm{H}$ & -3.90713800 & -4.75711600 & -1.33995500 \\
\hline $\mathrm{H}$ & 1.49756700 & 5.08285900 & -2.85186700 & $\mathrm{H}$ & -4.75411300 & -3.75067300 & -0.13836400 \\
\hline 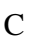 & -3.14215600 & 4.52413600 & 1.82931600 & $\mathrm{Z}$ & 0.66395200 & 4.35703400 & 2.42114800 \\
\hline $\mathrm{H}$ & -2.07445800 & 4.75208700 & 1.70151500 & $\mathrm{C}$ & 0.16626900 & 2.95781700 & 0.98570100 \\
\hline $\mathrm{H}$ & -3.31594100 & 4.32015100 & 2.89599600 & $\mathrm{H}$ & 0.45111800 & 2.11363700 & 1.61186600 \\
\hline $\mathrm{H}$ & -3.71346600 & 5.43214000 & 1.58144800 & $\mathrm{H}$ & -0.89139300 & 3.16016200 & 0.82007100 \\
\hline $\mathrm{C}$ & 5.47649300 & -3.13214200 & 1.63615100 & $\mathrm{H}$ & 0.89652300 & 3.39520200 & 0.30087000 \\
\hline 11 & 5.59451600 & -4.22282500 & 1.73226600 & $\mathrm{C}$ & 0.99316100 & 4.85656400 & 4.29104400 \\
\hline 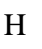 & 6.06881600 & -2.65877500 & 2.43479100 & $\mathrm{H}$ & 1.67843400 & 5.71798900 & 4.35641000 \\
\hline $\mathrm{H}$ & 5.91245100 & -2.84396800 & 0.66914700 & $\mathrm{H}$ & 0.05643700 & 5.13912900 & 4.79977400 \\
\hline C & 3.43930500 & -3.18190300 & 3.10110200 & $\mathrm{H}$ & 1.43840900 & 4.02946000 & 4.86496700 \\
\hline $\mathrm{H}$ & 2.35591700 & -3.01392400 & 3.17495400 & $\mathrm{O}$ & 0.15264600 & 6.11831600 & 1.35474200 \\
\hline $\mathrm{H}$ & 3.93082400 & -2.62829700 & 3.91586500 & $\mathrm{C}$ & -0.26121200 & 7.32111000 & 2.02444800 \\
\hline $\mathrm{H}$ & 3.62040100 & -4.25205100 & 3.28404600 & $\mathrm{C}$ & -0.10851700 & 6.20252500 & -0.05937700 \\
\hline $\mathrm{C}$ & -3.44361300 & 0.91279700 & -4.43334400 & $\mathrm{C}$ & -1.27781200 & 7.94191500 & 1.08485100 \\
\hline $\mathrm{H}$ & -4.42479300 & 0.89470900 & -3.93702800 & $\mathrm{H}$ & -0.65800100 & 7.04186400 & 3.00972800 \\
\hline $\mathrm{H}$ & -3.32016100 & -0.03239500 & -4.98404300 & $\mathrm{H}$ & 0.61724700 & 7.97278300 & 2.17128500 \\
\hline $\mathrm{H}$ & -3.46665700 & 1.73078000 & -5.17050300 & $\mathrm{C}$ & -0.69443800 & 7.59062300 & -0.28388700 \\
\hline $\mathrm{C}$ & -4.62706200 & 0.35696500 & 4.09267200 & $\mathrm{H}$ & 0.82979800 & 6.02972600 & -0.60008800 \\
\hline $\mathrm{H}$ & -5.47277400 & -0.25552300 & 3.74530200 & $\mathrm{H}$ & -0.81010000 & 5.40030900 & -0.32657200 \\
\hline $\mathrm{H}$ & -4.97388300 & 1.39721700 & 4.18985400 & $\mathrm{H}$ & -1.39743600 & 9.02240300 & 1.24397600 \\
\hline $\mathrm{H}$ & -4.35569900 & 0.00734900 & 5.10047000 & $\mathrm{H}$ & -2.26090100 & 7.46645000 & 1.22422800 \\
\hline $\mathrm{C}$ & 5.14124700 & 1.05343700 & 2.20143900 & $\mathrm{H}$ & 0.09605100 & 8.30708500 & -0.55662400 \\
\hline $\mathrm{H}$ & 5.58340000 & 0.88455000 & 1.20993600 & $\mathrm{H}$ & -1.44077200 & 7.59866900 & -1.09037200 \\
\hline $\mathrm{H}$ & 5.32364000 & 0.15571500 & 2.81154700 & & & & \\
\hline $\mathrm{H}$ & 5.68159000 & 1.89388500 & 2.66400600 & \multicolumn{2}{|c|}{ TS3 } & & \\
\hline $\mathrm{C}$ & 3.04661400 & 1.54563000 & 3.50919800 & $\mathrm{P}$ & -0.18603400 & -1.16068700 & -0.43147500 \\
\hline $\mathrm{H}$ & 3.58870400 & 2.30365800 & 4.09475100 & $\mathrm{P}$ & -0.03669300 & 1.45213500 & 0.30311500 \\
\hline $\mathrm{H}$ & 3.09796600 & 0.60244500 & 4.07228000 & $\mathrm{~N}$ & 3.23404000 & -0.66006200 & -1.00336500 \\
\hline $\mathrm{H}$ & 1.99143600 & 1.85187800 & 3.46371500 & $\mathrm{~N}$ & -3.38899300 & 1.41592300 & 0.39999100 \\
\hline $\mathrm{C}$ & -0.95270600 & 1.16869800 & -4.16313100 & $\mathrm{~N}$ & 3.19072500 & 1.32906500 & 0.00056700 \\
\hline $\mathrm{H}$ & -0.90477700 & 2.01312200 & -4.86710900 & $\mathrm{~N}$ & -3.49754700 & -0.78318300 & 0.62975100 \\
\hline $\mathrm{H}$ & -0.80098000 & 0.25263600 & -4.75205600 & $\mathrm{C}$ & -2.64208000 & 0.25866700 & 0.40958000 \\
\hline $\mathrm{H}$ & -0.12239000 & 1.26692100 & -3.44901600 & $\mathrm{C}$ & 2.42486300 & 0.23572600 & -0.35655900 \\
\hline $\mathrm{C}$ & -5.09624200 & 3.12048600 & 1.15288500 & $\mathrm{C}$ & 2.96272400 & 2.07948300 & 1.19072200 \\
\hline $\mathrm{H}$ & -5.65367100 & 3.96836500 & 0.72538500 & $\mathrm{C}$ & 3.14678800 & -3.10471300 & -1.01981200 \\
\hline $\mathrm{H}$ & -5.36185800 & 3.04173900 & 2.21783000 & $\mathrm{C}$ & 2.78460200 & -1.87086400 & -1.60108500 \\
\hline $\mathrm{H}$ & -5.44416900 & 2.20676600 & 0.65687600 & $\mathrm{C}$ & 2.96544600 & 1.42481600 & 2.44442000 \\
\hline $\mathrm{C}$ & -1.35190100 & -4.52301100 & -0.30016700 & $\mathrm{C}$ & -3.12247300 & -2.09440400 & 1.03128000 \\
\hline$\Pi$ & -0.42216400 & -3.98402300 & -0.07678300 & $\mathrm{C}$ & 1.07865700 & 0.12563900 & -0.10850200 \\
\hline $\mathrm{H}$ & -1.32620600 & -4.83210100 & -1.35574800 & $\mathrm{C}$ & 4.62833600 & -0.22451300 & -0.99487100 \\
\hline $\mathrm{H}$ & -1.37801100 & -5.44353800 & 0.30377700 & $\mathrm{H}$ & 5.08883800 & -0.38688300 & -1.97932900 \\
\hline
\end{tabular}




\begin{tabular}{|c|c|c|c|c|c|c|c|}
\hline $\mathrm{H}$ & 5.21984600 & -0.77788600 & -0.24810200 & $\mathrm{H}$ & 2.64913800 & 3.38108800 & -1.00836600 \\
\hline $\mathrm{C}$ & 2.76920700 & 3.47036400 & 1.09301600 & $\mathrm{C}$ & -4.01122000 & 1.52332100 & -2.41394000 \\
\hline $\mathrm{C}$ & 1.96945000 & -1.80533400 & -2.75328600 & $\mathrm{H}$ & -4.59893500 & 0.96463900 & -1.67184800 \\
\hline $\mathrm{C}$ & -2.85986800 & -2.33170400 & 2.39635100 & $\mathrm{C}$ & -2.48128100 & 4.95439900 & -1.67788200 \\
\hline $\mathrm{C}$ & -1.27527000 & 0.16768400 & 0.23544100 & $\mathrm{H}$ & -2.26980900 & 5.88464300 & -2.21037500 \\
\hline $\mathrm{C}$ & 4.50620400 & 1.25462800 & -0.62999900 & $\mathrm{C}$ & 0.20261600 & -2.37873900 & 0.89448900 \\
\hline $\mathrm{H}$ & 5.28718000 & 1.58765500 & 0.06613600 & $\mathrm{H}$ & -0.57577400 & -3.14552600 & 0.97039400 \\
\hline $\mathrm{H}$ & 4.54927600 & 1.89884200 & -1.52630200 & $\mathrm{H}$ & 0.36851400 & -1.90157100 & 1.86740800 \\
\hline $\mathrm{C}$ & -3.05349100 & 2.60263200 & -0.30784300 & $\mathrm{H}$ & 1.12717600 & -2.86926300 & 0.56251200 \\
\hline $\mathrm{C}$ & 4.04485900 & -3.18037700 & 0.20159600 & $\mathrm{C}$ & 1.86736000 & -4.23178000 & -2.74787000 \\
\hline $\mathrm{H}$ & 4.04063800 & -2.18356100 & 0.66670300 & $\mathrm{H}$ & 1.50237800 & -5.15857000 & -3.19639400 \\
\hline $\mathrm{C}$ & -3.36687000 & 2.68732900 & -1.68233100 & $\mathrm{C}$ & -2.78360300 & -4.41716200 & 0.50787700 \\
\hline $\mathrm{C}$ & 3.26874700 & -0.05543900 & 2.58041600 & $\mathrm{H}$ & -2.75456500 & -5.23613500 & -0.21361700 \\
\hline $\mathrm{H}$ & 3.00674000 & -0.54288600 & 1.63306700 & $\mathrm{C}$ & 2.42934800 & -0.74443900 & 3.65313900 \\
\hline $\mathrm{C}$ & -2.49256600 & 3.68845500 & 0.39697000 & $\mathrm{H}$ & 2.71340500 & -0.43295500 & 4.67011000 \\
\hline $\mathrm{C}$ & -3.09300100 & -3.12406000 & 0.07159100 & $\mathrm{H}$ & 2.56594400 & -1.83478600 & 3.59829300 \\
\hline $\mathrm{C}$ & 2.75051300 & 2.19783700 & 3.58827900 & $\mathrm{H}$ & 1.36136000 & -0.52632300 & 3.51634400 \\
\hline $\mathrm{H}$ & 2.74400300 & 1.71495500 & 4.56692200 & $\mathrm{C}$ & -1.49888100 & -0.71068700 & 3.75425100 \\
\hline $\mathrm{C}$ & -2.32239900 & 3.62461500 & 1.90148200 & $\mathrm{H}$ & -0.89188100 & -1.51621600 & 4.19664600 \\
\hline $\mathrm{H}$ & -2.28775800 & 2.55713900 & 2.17011400 & $\mathrm{H}$ & -1.54236300 & 0.11672000 & 4.47961100 \\
\hline $\mathrm{C}$ & 2.68028600 & -4.27860900 & -1.62062300 & $\mathrm{H}$ & -0.98965900 & -0.34856800 & 2.85040800 \\
\hline $\mathrm{H}$ & 2.94860800 & -5.24516400 & -1.18981700 & $\mathrm{C}$ & -3.42766500 & -2.85474000 & -1.38087400 \\
\hline $\mathrm{C}$ & -2.90653000 & -1.21175600 & 3.41918000 & $\mathrm{H}$ & -3.41491600 & -1.76271400 & -1.50739800 \\
\hline $\mathrm{H}$ & -3.44181600 & -0.36820300 & 2.95825900 & $\mathrm{C}$ & -4.88895300 & -0.34784100 & 0.68968500 \\
\hline $\mathrm{C}$ & -4.75170800 & 1.16426100 & 0.86651400 & $\mathrm{H}$ & -5.40326500 & -0.82964800 & 1.53316000 \\
\hline $\mathrm{H}$ & -5.47571700 & 1.73753700 & 0.27098200 & $\mathrm{H}$ & -5.42785700 & -0.62049200 & -0.23504800 \\
\hline $\mathrm{H}$ & -4.86600800 & 1.46537100 & 1.92228300 & $\mathrm{C}$ & 4.76649700 & -0.27008500 & 2.82248800 \\
\hline $\mathrm{C}$ & -2.19260400 & 4.85509200 & -0.31922700 & $\mathrm{H}$ & 5.37692500 & 0.18703500 & 2.02958400 \\
\hline $\mathrm{H}$ & -1.75400000 & 5.70733500 & 0.20190900 & $\mathrm{H}$ & 5.00717100 & -1.34399900 & 2.86216500 \\
\hline $\mathrm{C}$ & 1.62840900 & -0.47088000 & -3.39037800 & $\mathrm{H}$ & 5.07853800 & 0.18213200 & 3.77679700 \\
\hline $\mathrm{H}$ & 1.47093700 & 0.23777800 & -2.56587500 & $\mathrm{C}$ & 5.48173200 & -3.51741000 & -0.21674600 \\
\hline $\mathrm{C}$ & -2.54197400 & -3.63710500 & 2.78200600 & $\mathrm{H}$ & 5.53145000 & -4.53557700 & -0.63345600 \\
\hline $\mathrm{H}$ & -2.32212600 & -3.85202600 & 3.82960300 & $\mathrm{H}$ & 6.16383400 & -3.47320100 & 0.64656500 \\
\hline $\mathrm{C}$ & 1.51614100 & -3.00609400 & -3.30598300 & $\mathrm{H}$ & 5.85925300 & -2.83275600 & -0.98987000 \\
\hline $\mathrm{H}$ & 0.87414400 & -2.98539300 & -4.18687900 & $\mathrm{C}$ & 3.55941600 & -4.16629100 & 1.26530700 \\
\hline $\mathrm{C}$ & -3.06986900 & 3.88310600 & -2.35447900 & $\mathrm{H}$ & 2.53143000 & -3.95061200 & 1.58747700 \\
\hline $\mathrm{H}$ & -3.35134700 & 3.99604600 & -3.40260800 & $\mathrm{H}$ & 4.20968700 & -4.11134400 & 2.15171600 \\
\hline $\mathrm{C}$ & 2.57468200 & 3.57705900 & 3.50773300 & $\mathrm{H}$ & 3.59083800 & -5.20656300 & 0.90730700 \\
\hline $\mathrm{H}$ & 2.42414700 & 4.16245700 & 4.41765300 & $\mathrm{C}$ & -1.03748400 & 4.27115100 & 2.40869400 \\
\hline $\mathrm{C}$ & 2.59385800 & 4.20663100 & 2.26958100 & $\mathrm{H}$ & -0.15396600 & 3.81176900 & 1.94607200 \\
\hline $\mathrm{H}$ & 2.45182000 & 5.28764500 & 2.21263900 & $\mathrm{H}$ & -0.95271100 & 4.13265700 & 3.49673500 \\
\hline $\mathrm{C}$ & -2.50831900 & -4.67118900 & 1.84864800 & $\mathrm{H}$ & -1.01381300 & 5.35572400 & 2.21879500 \\
\hline $\mathrm{H}$ & -2.26369700 & -5.68594600 & 2.17106900 & $\mathrm{C}$ & 4.05611800 & 4.91994100 & -0.50890800 \\
\hline $\mathrm{C}$ & 2.74549300 & 4.17257600 & -0.24760400 & $\mathrm{H}$ & 4.20759900 & 5.71515400 & 0.23765200 \\
\hline
\end{tabular}




\begin{tabular}{|c|c|c|c|c|c|c|c|}
\hline $\mathrm{H}$ & 4.05120600 & 5.39401300 & -1.50367200 & $\mathrm{H}$ & -1.87157000 & 4.01277200 & -5.67521500 \\
\hline $\mathrm{H}$ & 4.92597300 & 4.24905700 & -0.45499800 & $\mathrm{H}$ & -0.43749100 & 3.07478400 & -6.14541700 \\
\hline$C$ & 1.53791200 & 5.10031100 & -0.37908100 & $\mathrm{H}$ & -1.92415900 & 2.23762200 & -5.65540400 \\
\hline $\mathrm{H}$ & 0.61208900 & 4.57651000 & -0.10767200 & $\mathrm{C}$ & 2.03737000 & 4.51700600 & -4.07011200 \\
\hline $\mathrm{H}$ & 1.42868200 & 5.45867600 & -1.41146300 & $\mathrm{O}$ & 0.71173000 & 4.84193000 & -3.61345000 \\
\hline $\mathrm{H}$ & 1.62979100 & 5.97966600 & 0.27644800 & $\mathrm{C}$ & 0.28019200 & 6.10143300 & -4.16499500 \\
\hline $\mathrm{C}$ & -4.97181700 & 1.96701700 & -3.51558500 & $\mathrm{C}$ & 1.31831500 & 6.45475200 & -5.21756100 \\
\hline $\mathrm{H}$ & -5.70548600 & 2.70004800 & -3.14805900 & $\mathrm{C}$ & 2.58142800 & 5.81130100 & -4.64735600 \\
\hline $\mathrm{H}$ & -5.52337200 & 1.09861100 & -3.90512800 & $\mathrm{H}$ & 2.60857000 & 4.12844500 & -3.21538800 \\
\hline $\mathrm{H}$ & -4.43779800 & 2.41474900 & -4.36784000 & $\mathrm{H}$ & 1.96596800 & 3.72717800 & -4.83916000 \\
\hline $\mathrm{C}$ & -3.67874500 & -1.60420400 & 4.67920500 & $\mathrm{H}$ & -0.73382900 & 5.97415900 & -4.57065400 \\
\hline $\mathrm{H}$ & -4.68121800 & -1.98787600 & 4.43608200 & $\mathrm{H}$ & 0.24772800 & 6.83904500 & -3.34610500 \\
\hline $\mathrm{H}$ & -3.79600000 & -0.73347200 & 5.34225800 & $\mathrm{H}$ & 1.05523200 & 5.99475900 & -6.18295100 \\
\hline $\mathrm{H}$ & -3.15402900 & -2.38115800 & 5.25610300 & $\mathrm{H}$ & 1.40968600 & 7.53862000 & -5.37169600 \\
\hline $\mathrm{C}$ & 2.79447500 & 0.04983900 & -4.23541200 & $\mathrm{H}$ & 3.36411500 & 5.64015100 & -5.39910200 \\
\hline $\mathrm{H}$ & 3.71773700 & 0.13985700 & -3.64583500 & $\mathrm{H}$ & 3.00890500 & 6.43685900 & -3.84766200 \\
\hline $\mathrm{H}$ & 2.55860000 & 1.04369500 & -4.64940400 & & & & \\
\hline $\mathrm{H}$ & 3.00244200 & -0.62547700 & -5.07983800 & $\mathrm{Zn}$ & $(\mathrm{Me})_{2}(\mathrm{thf})$ & & \\
\hline $\mathrm{C}$ & 0.33626300 & -0.49589100 & -4.19879600 & $\mathrm{Zn}$ & -0.46420600 & -0.28913400 & -0.80102100 \\
\hline $\mathrm{H}$ & 0.43280900 & -1.08413400 & -5.12446200 & $\mathrm{C}$ & -0.51052100 & 0.18323500 & 1.10995700 \\
\hline $\mathrm{H}$ & 0.06022900 & 0.52614900 & -4.50252500 & $\mathrm{H}$ & 0.30791000 & 0.87262500 & 1.382 \\
\hline $\mathrm{H}$ & -0.48842600 & -0.91337500 & -3.60393300 & $\mathrm{H}$ & -1.45609400 & 0.68396700 & 1.38441900 \\
\hline $\mathrm{C}$ & -2.96120400 & 0.55622600 & -2.96718800 & $\mathrm{H}$ & -0.41710500 & -0.70183800 & 1.76087100 \\
\hline $\mathrm{H}$ & -2.33581300 & 1.05592100 & -3.72096200 & $\mathrm{C}$ & -0.40135200 & -1.32430100 & -2.47484900 \\
\hline $\mathrm{H}$ & -3.44646400 & -0.29963100 & -3.45905500 & $\mathrm{H}$ & 0.52360700 & -1.12429500 & -3.04460600 \\
\hline $\mathrm{H}$ & -2.30440000 & 0.16341200 & -2.17890400 & $\mathrm{H}$ & -0.43664100 & -2.41148900 & -2.29442700 \\
\hline $\mathrm{C}$ & -3.55598200 & 4.24409000 & 2.57225500 & $\mathrm{H}$ & -1.24724300 & -1.07977500 & -3.14121600 \\
\hline $\mathrm{H}$ & -3.62199600 & 5.32088600 & 2.34962600 & $\mathrm{C}$ & -0.51701400 & 2.96912300 & -0.89437400 \\
\hline $\mathrm{H}$ & -3.50100000 & 4.12806400 & 3.66550300 & $\mathrm{O}$ & -0.52345700 & 1.76449300 & -1.66622000 \\
\hline $\mathrm{H}$ & -4.49020700 & 3.77902600 & 2.22503700 & $\mathrm{C}$ & -0.57677400 & 2.05106600 & -3.06692700 \\
\hline $\mathrm{C}$ & -2.39438400 & -3.43723000 & -2.34522500 & $\mathrm{C}$ & -0.29108000 & 3.54169000 & -3.17911100 \\
\hline $\mathrm{H}$ & -1.38618700 & -3.06272200 & -2.11259300 & $\mathrm{C}$ & -0.88619700 & 4.07214400 & -1.87577600 \\
\hline $\mathrm{H}$ & -2.63655200 & -3.14840200 & -3.37997300 & $\mathrm{H}$ & -1.22351200 & 2.85655700 & -0.05996500 \\
\hline $\mathrm{H}$ & -2.37539100 & -4.53763100 & -2.31119800 & $\mathrm{H}$ & 0.49128000 & 3.12028100 & -0.47032500 \\
\hline $\mathrm{C}$ & -4.84076300 & -3.35066600 & -1.70574000 & $\mathrm{H}$ & 0.15431600 & 1.41435300 & -3.58477500 \\
\hline $\mathrm{H}$ & -4.90485600 & -4.44637900 & -1.61321300 & $\mathrm{H}$ & -1.58145500 & 1.79774800 & -3.44868100 \\
\hline $\mathrm{H}$ & -5.12129400 & -3.08466100 & -2.73700000 & $\mathrm{H}$ & 0.79548800 & 3.72400600 & -3.20617600 \\
\hline $\mathrm{H}$ & -5.59024300 & -2.92040900 & -1.02434400 & $\mathrm{H}$ & -0.73126300 & 3.99132800 & -4.08052200 \\
\hline $\mathrm{Zn}$ & -0.63027100 & 3.14200100 & -3.58664900 & $\mathrm{H}$ & -0.49206900 & 5.05425400 & -1.57811900 \\
\hline $\mathrm{C}$ & -0.07371500 & 2.33818200 & -1.75335300 & $\mathrm{H}$ & -1.98144200 & 4.15914700 & -1.96223600 \\
\hline $\mathrm{H}$ & -0.64093200 & 3.17252300 & -1.33688500 & & & & \\
\hline $\mathrm{H}$ & -0.55543800 & 1.40527500 & -2.04245800 & & $(\mathrm{Me})(\mathrm{thf})(\mathrm{I})$ & & \\
\hline $\mathrm{H}$ & 1.01083700 & 2.42512300 & -1.81436500 & $\mathrm{Zn}$ & -0.19603300 & -0.30469500 & -0.98847900 \\
\hline $\mathrm{C}$ & -1.28577800 & 3.10800300 & -5.44171300 & $\mathrm{C}$ & -1.21439000 & -0.39594200 & 0.68529100 \\
\hline
\end{tabular}




\begin{tabular}{|c|c|c|c|c|c|c|c|}
\hline $\mathrm{H}$ & -1.13046800 & 0.54405500 & 1.25617800 & $\mathrm{H}$ & 5.38673200 & 0.69170500 & 1.05591100 \\
\hline $\mathrm{H}$ & -2.28328400 & -0.57056900 & 0.48835800 & $\mathrm{H}$ & 5.30891500 & 0.67935300 & -0.72143500 \\
\hline $\mathrm{H}$ & -0.85338700 & -1.21276900 & 1.33211300 & $\mathrm{C}$ & -3.05376600 & 2.48003300 & -0.10723500 \\
\hline $\mathrm{C}$ & 2.96726800 & -0.06439000 & -1.38422900 & $\mathrm{C}$ & 2.58100600 & -3.84080600 & 1.03401500 \\
\hline 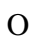 & 1.85058500 & -0.19588200 & -0.48255100 & $\mathrm{H}$ & 2.63277500 & -2.83317700 & 1.47701000 \\
\hline$C$ & 2.28878600 & -0.23608200 & 0.88496400 & $\mathrm{C}$ & -3.33575300 & 2.44539900 & -1.48992900 \\
\hline$c$ & 3.74196800 & 0.20738800 & 0.84056300 & $\mathrm{C}$ & 2.40623900 & 1.40134100 & 2.89168100 \\
\hline $\mathrm{C}$ & 4.19096000 & -0.32218800 & -0.52053200 & $\mathrm{H}$ & 2.16448900 & 0.46969700 & 2.35764300 \\
\hline $\mathrm{H}$ & 2.82993300 & -0.77528100 & -2.20991800 & $\mathrm{C}$ & -2.62528600 & 3.65440800 & 0.53933400 \\
\hline $\mathrm{H}$ & 2.95816800 & 0.95420300 & -1.80553400 & $\mathrm{C}$ & -3.33669700 & -3.14739200 & -0.04466200 \\
\hline 14 & 1.63561400 & 0.41737000 & 1.48020900 & $\mathrm{C}$ & 2.39070600 & 3.82684800 & 2.14440600 \\
\hline $\mathrm{H}$ & 2.18443800 & -1.26784500 & 1.26203900 & $\mathrm{H}$ & 2.01238100 & 4.10219300 & 3.12897700 \\
\hline $\mathrm{H}$ & 3.80848600 & 1.30691200 & 0.86663400 & $\mathrm{C}$ & -2.38511500 & 3.69000300 & 2.03395600 \\
\hline $\mathrm{H}$ & 4.32796900 & -0.18710000 & 1.68264800 & $\mathrm{H}$ & -2.32761900 & 2.64252900 & 2.37000200 \\
\hline 11 & 5.09018300 & 0.17542900 & -0.90994700 & $\mathrm{C}$ & 2.19268900 & -4.72134600 & -1.31037700 \\
\hline . & 4.40079700 & -1.40235000 & -0.46221800 & $\mathrm{H}$ & 1.95665400 & -5.69619600 & -0.87918800 \\
\hline \multirow[t]{2}{*}{ I } & -0.09790800 & -0.24049600 & -3.49386000 & $\mathrm{C}$ & -1.84461600 & -1.73250000 & 3.23310100 \\
\hline & & & & $\mathrm{H}$ & -2.04983100 & -0.70873500 & 2.88516500 \\
\hline 1 & & & & $\mathrm{C}$ & -4.63669000 & 1.02475500 & 1.13864500 \\
\hline \multirow[t]{2}{*}{ I } & 0.72399500 & .01773100 & 0.00000000 & $\mathrm{H}$ & -5.34883500 & 1.10030300 & 0.29741700 \\
\hline & & & & $\mathrm{H}$ & -4.94589100 & 1.7367 & 1.91499700 \\
\hline \multicolumn{2}{|c|}{$\operatorname{cis}-\mathbf{3} \mathbf{a}^{\bullet+}$} & & & $\mathrm{C}$ & -2.46038400 & 4.80680600 & -0.23731100 \\
\hline $\mathrm{P}$ & -0.12817000 & -1.25258300 & -0.13417600 & $\mathrm{H}$ & -2.12544400 & 5.73103600 & 0.23707100 \\
\hline P & 0.03979100 & 1.38661900 & 0.24614500 & $\mathrm{C}$ & 3.18890400 & -0.90621500 & -3.09757600 \\
\hline $\mathrm{N}$ & 3.23560100 & -1.33800000 & -0.22486300 & $\mathrm{H}$ & 3.51634900 & -0.22995100 & -2.29622000 \\
\hline $\mathrm{N}$ & -3.27999800 & 1.30693400 & 0.67336900 & $\mathrm{C}$ & -2.00338000 & -4.05892200 & 2.26116300 \\
\hline $\mathrm{N}$ & 3.43232800 & 0.81226600 & 0.26069600 & $\mathrm{H}$ & -1.48486200 & -4.42684400 & 3.14868200 \\
\hline $\mathrm{N}$ & -3.36978600 & -0.89878800 & 0.88286200 & $\mathrm{C}$ & 2.46364400 & -3.31832500 & -3.25750400 \\
\hline 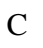 & -2.57582600 & 0.14619100 & 0.49861300 & $\mathrm{H}$ & 2.44067100 & -3.20281900 & -4.34344000 \\
\hline $\mathrm{C}$ & 2.53986600 & -0.17086500 & -0.05205200 & $\mathrm{C}$ & -3.15080200 & 3.62113400 & -2.22336500 \\
\hline$C$ & 3.12621900 & 2.16892700 & 0.56585600 & $\mathrm{H}$ & -3.35134000 & 3.62236600 & -3.29696900 \\
\hline $\mathrm{C}$ & 2.52296000 & -3.65457900 & -0.46687400 & $\mathrm{C}$ & 2.63063200 & 4.82329900 & 1.20238700 \\
\hline $\mathrm{C}$ & 2.82577200 & -2.41268400 & -1.05634600 & $\mathrm{H}$ & 2.43658300 & 5.86799600 & 1.45581800 \\
\hline$c$ & 2.62303700 & 2.48144100 & 1.84713200 & $C$ & 3.11972300 & 4.49443900 & -0.05724600 \\
\hline $\mathrm{C}$ & -2.96338300 & -2.25821700 & 0.98894400 & $\mathrm{H}$ & 3.30802400 & 5.28549700 & -0.78582700 \\
\hline $\mathrm{C}$ & 1.16250600 & -0.03847100 & -0.15777700 & $\mathrm{C}$ & -2.37945200 & -4.95381500 & 1.26622800 \\
\hline $\mathrm{C}$ & 4.62628900 & -1.18706100 & 0.19753800 & $\mathrm{H}$ & -2.15884200 & -6.01759900 & 1.38010200 \\
\hline $\mathrm{H}$ & 5.29722200 & -1.70054700 & -0.50385900 & $\mathrm{C}$ & 3.98941800 & 2.83403100 & -1.75318100 \\
\hline$\Pi$ & 4.77665900 & -1.62047900 & 1.20116600 & $\mathrm{H}$ & 3.88839400 & 1.74761400 & -1.89786500 \\
\hline $\mathrm{C}$ & 3.38286500 & 3.16411300 & -0.40093700 & $\mathrm{C}$ & -3.82370200 & 1.19113900 & -2.19142300 \\
\hline $\mathrm{C}$ & 2.81411800 & -2.22778900 & -2.45644300 & $\mathrm{H}$ & -4.01180500 & 0.42449400 & -1.42695100 \\
\hline$C$ & -2.27293200 & -2.69160400 & 2.13856700 & $\mathrm{C}$ & -2.71749100 & 4.79155700 & -1.60509700 \\
\hline $\mathrm{C}$ & -1.26577000 & 0.08980000 & 0.04541100 & $\mathrm{H}$ & -2.58081000 & 5.70046500 & -2.19547600 \\
\hline $\mathrm{C}$ & 4.81017200 & 0.32670900 & 0.19561200 & $\mathrm{C}$ & -0.44040100 & -2.22071300 & -1.65395700 \\
\hline
\end{tabular}




\begin{tabular}{|c|c|c|c|c|c|c|c|}
\hline $\mathrm{H}$ & -0.34973100 & -1.58101800 & -2.54024300 & $\mathrm{C}$ & 3.28304900 & 3.50648900 & -2.93136800 \\
\hline $\mathrm{H}$ & -1.46290000 & -2.60012500 & -1.56467700 & $\mathrm{H}$ & 2.21791300 & 3.24363800 & -2.97919800 \\
\hline $\mathrm{H}$ & 0.24822400 & -3.06956100 & -1.71861200 & $\mathrm{H}$ & 3.75016600 & 3.19161400 & -3.87690900 \\
\hline $\mathrm{C}$ & 2.15931000 & -4.55499400 & -2.69262300 & $\mathrm{H}$ & 3.35997000 & 4.60330600 & -2.88407100 \\
\hline $\mathrm{H}$ & 1.89718000 & -5.39773300 & -3.33644300 & $\mathrm{C}$ & -5.14961400 & 1.42592300 & -2.91830600 \\
\hline C & -3.02657000 & -4.50029300 & 0.11843700 & $\mathrm{H}$ & -5.91470700 & 1.83126500 & -2.23897900 \\
\hline $\mathrm{H}$ & -3.29930000 & -5.21514900 & -0.65878700 & $\mathrm{H}$ & -5.52933300 & 0.48153200 & -3.33752500 \\
\hline $\mathrm{C}$ & 1.23225700 & 1.69162400 & 3.82254600 & $\mathrm{H}$ & -5.03795400 & 2.13464600 & -3.75301200 \\
\hline $\mathrm{H}$ & 1.40772500 & 2.57875400 & 4.44987600 & $\mathrm{C}$ & -2.64821400 & -1.97223200 & 4.51567400 \\
\hline $\mathrm{H}$ & 1.07870300 & 0.84438600 & 4.50625700 & $\mathrm{H}$ & -3.73264100 & -1.92389400 & 4.33546600 \\
\hline $\mathrm{H}$ & 0.30871900 & 1.84694500 & 3.24691000 & $\mathrm{H}$ & -2.39287900 & -1.22280900 & 5.28045100 \\
\hline $\mathrm{C}$ & 0.08404200 & 2.29637100 & -1.36256400 & $\mathrm{H}$ & -2.43209400 & -2.96639200 & 4.93679600 \\
\hline $\mathrm{H}$ & 1.03338800 & 2.84557600 & -1.36728000 & $\mathrm{C}$ & 4.36721300 & -1.06016100 & -4.06155600 \\
\hline $\mathrm{H}$ & -0.73475400 & 3.02407000 & -1.38956000 & $\mathrm{H}$ & 5.23062000 & -1.53152700 & -3.56819000 \\
\hline $\mathrm{H}$ & 0.02957300 & 1.63225700 & -2.23349400 & $\mathrm{H}$ & 4.68382500 & -0.07613700 & -4.44035500 \\
\hline$C$ & -0.34158400 & -1.80760400 & 3.50681900 & $\mathrm{H}$ & 4.10345300 & -1.67896600 & -4.93290600 \\
\hline $\mathrm{H}$ & -0.04571600 & -2.79857000 & 3.88174600 & $\mathrm{C}$ & 1.98611000 & -0.24363300 & -3.77051700 \\
\hline$\Pi$ & -0.05972800 & -1.06770900 & 4.26918800 & $\mathrm{H}$ & 1.58299700 & -0.86895200 & -4.58242200 \\
\hline$H$ & 0.23892500 & -1.60058800 & 2.59579000 & $\mathrm{H}$ & 2.27012400 & 0.72716800 & -4.20379300 \\
\hline$C$ & -4.05336500 & -2.64502800 & -1.28860100 & $\mathrm{H}$ & 1.18649500 & -0.06414900 & -3.03884100 \\
\hline $\mathrm{H}$ & -3.63983400 & -1.64667800 & -1.50457800 & $\mathrm{C}$ & -2.75608900 & 0.62491600 & -3.13031700 \\
\hline $\mathrm{C}$ & -4.50720600 & -0.39877800 & 1.65546500 & $\mathrm{H}$ & -2.49789100 & 1.34262300 & -3.92432100 \\
\hline H & -4.27369500 & -0.41732200 & 2.73377700 & $\mathrm{H}$ & -3.11432900 & -0.29684900 & -3.61441900 \\
\hline $\mathrm{H}$ & -5.40226900 & -1.01116100 & 1.49248200 & $\mathrm{H}$ & -1.84197400 & 0.38647300 & -2.56999500 \\
\hline$C$ & 3.69491600 & 1.16645200 & 3.68748500 & $\mathrm{C}$ & -3.56040200 & 4.36200700 & 2.75234400 \\
\hline$H$ & 4.53851600 & 0.90549300 & 3.03239200 & $\mathrm{H}$ & -3.64696400 & 5.41987000 & 2.45807300 \\
\hline $\mathrm{H}$ & 3.56285500 & 0.34767500 & 4.41168800 & $\mathrm{H}$ & -3.42197400 & 4.32706600 & 3.84404800 \\
\hline П & 3.97962700 & 2.07158600 & 4.24624800 & $\mathrm{H}$ & -4.51970800 & 3.87850800 & 2.51395900 \\
\hline 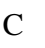 & 3.84466300 & -4.60795000 & 1.43634000 & $\mathrm{C}$ & -3.82879400 & -3.52037800 & -2.52139600 \\
\hline $\mathrm{H}$ & 3.82833300 & -5.63151900 & 1.02992300 & $\mathrm{H}$ & -2.76450000 & -3.72605800 & -2.70757400 \\
\hline П & 3.92439800 & -4.68256800 & 2.53183700 & $\mathrm{H}$ & -4.23455200 & -3.02009800 & -3.41313900 \\
\hline$H$ & 4.75588400 & -4.12022100 & 1.05934600 & $\mathrm{H}$ & -4.34626000 & -4.48800300 & -2.43357400 \\
\hline $\mathrm{C}$ & 1.32076000 & -4.51094700 & 1.57270300 & $\mathrm{C}$ & -5.55807600 & -2.47642800 & -1.05192000 \\
\hline 11 & 0.42226900 & -3.95170800 & 1.27808000 & $\mathrm{H}$ & -6.01905500 & -3.43785900 & -0.77788900 \\
\hline $\mathrm{H}$ & 1.34925300 & -4.55572500 & 2.67168200 & $\mathrm{H}$ & -6.05193200 & -2.11143100 & -1.96529200 \\
\hline $\mathrm{H}$ & 1.21352600 & -5.54372300 & 1.20675500 & $\mathrm{H}$ & -5.77516100 & -1.75964000 & -0.25093200 \\
\hline $\mathrm{C}$ & -1.06052500 & 4.36105200 & 2.38710300 & & & & \\
\hline $\mathrm{H}$ & -0.22957400 & 3.88346500 & 1.85044400 & \multicolumn{2}{|c|}{ TS4 } & & \\
\hline $\mathrm{H}$ & -0.86583900 & 4.27842000 & 3.46704700 & $\mathrm{P}$ & -0.15790400 & -1.25516800 & 0.03070400 \\
\hline $\mathrm{H}$ & -1.06309600 & 5.43356100 & 2.13815700 & $\mathrm{P}$ & 0.17411100 & 1.31744400 & 0.01268800 \\
\hline $\mathrm{C}$ & 5.48163000 & 3.19217600 & -1.76062300 & $\mathrm{~N}$ & 3.28766000 & -1.41112000 & -0.71950000 \\
\hline$\Pi$ & 5.61547700 & 4.28305200 & -1.69253000 & $\mathrm{~N}$ & -3.16750400 & 1.57005600 & 0.45279400 \\
\hline $\mathrm{H}$ & 5.96287600 & 2.85224400 & -2.69076500 & $\mathrm{~N}$ & 3.43664300 & 0.79094800 & -0.41913700 \\
\hline $\mathrm{H}$ & 6.01978700 & 2.74816600 & -0.91071400 & $\mathrm{~N}$ & -3.50623200 & -0.58011400 & 0.81713600 \\
\hline
\end{tabular}




\begin{tabular}{|c|c|c|c|c|c|c|c|}
\hline $\mathrm{C}$ & -2.56530800 & 0.33956400 & 0.44570800 & $\mathrm{H}$ & 1.01684800 & -4.25809200 & -3.53838000 \\
\hline $\mathrm{C}$ & 2.56933100 & -0.28577200 & -0.40331500 & $\mathrm{C}$ & -2.43071500 & 4.17016700 & -2.07153500 \\
\hline $\mathrm{C}$ & 3.23947700 & 2.00867200 & 0.30385800 & $\mathrm{H}$ & -2.64387200 & 4.39916500 & -3.11697700 \\
\hline $\mathrm{C}$ & 2.97054900 & -3.75199200 & -0.19764300 & $\mathrm{C}$ & 3.02238300 & 4.40352200 & 1.70870300 \\
\hline 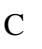 & 2.73709900 & -2.67026800 & -1.07422100 & $\mathrm{H}$ & 2.93167400 & 5.34179100 & 2.26054700 \\
\hline $\mathrm{C}$ & 3.13306900 & 1.97682200 & 1.71212500 & $\mathrm{C}$ & 3.14500900 & 4.41854500 & 0.32450600 \\
\hline $\mathrm{C}$ & -3.32894700 & -1.97495800 & 0.98969200 & $\mathrm{H}$ & 3.16118700 & 5.37377400 & -0.20328000 \\
\hline $\mathrm{C}$ & 1.21786400 & -0.21376700 & -0.13542100 & $\mathrm{C}$ & -3.19726500 & -4.71847000 & 1.39145500 \\
\hline $\mathrm{C}$ & 4.62916600 & -1.06153900 & -1.17068200 & $\mathrm{H}$ & -3.15167400 & -5.79806100 & 1.55240200 \\
\hline $\mathrm{H}$ & 4.66933600 & -1.03251200 & -2.27422100 & $\mathrm{C}$ & 3.51926500 & 3.29350700 & -1.89827100 \\
\hline 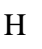 & 5.36937100 & -1.79573400 & -0.82189700 & $\mathrm{H}$ & 3.32233300 & 2.28624500 & -2.30124800 \\
\hline $\mathrm{C}$ & 3.27197300 & 3.23043900 & -0.40320400 & $\mathrm{C}$ & -3.81965800 & 2.05425900 & -2.29667000 \\
\hline 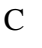 & 2.04420200 & -2.82740000 & -2.29004600 & $\mathrm{H}$ & -4.43838500 & 1.49617500 & -1.57696200 \\
\hline $\mathrm{C}$ & -2.93380100 & -2.46149500 & 2.25239500 & $\mathrm{C}$ & -1.62455300 & 5.03340500 & -1.33078600 \\
\hline $\mathrm{C}$ & -1.23801700 & 0.10664400 & 0.12587000 & $\mathrm{H}$ & -1.20783100 & 5.92527200 & -1.80471700 \\
\hline $\mathrm{C}$ & 4.81412700 & 0.31483700 & -0.55941800 & $\mathrm{C}$ & -0.36687500 & -3.04679100 & 0.05804900 \\
\hline $\mathrm{H}$ & 5.29732500 & 0.26243300 & 0.43273700 & $\mathrm{H}$ & -0.83414800 & -3.41123000 & -0.86431200 \\
\hline$\Pi$ & 5.40409100 & 0.98913100 & -1.19176200 & $\mathrm{H}$ & -0.96694500 & -3.33922900 & 0.92759400 \\
\hline $\mathrm{C}$ & -2.65004200 & 2.75256300 & -0.14104700 & $\mathrm{H}$ & 0.63652800 & -3.47668400 & 0.14744000 \\
\hline $\mathrm{C}$ & 3.64530800 & -3.51664100 & 1.14072400 & $\mathrm{C}$ & 1.78475300 & -5.18454700 & -1.75683200 \\
\hline $\mathrm{H}$ & 4.35348400 & -2.68471100 & 1.00519200 & $\mathrm{H}$ & 1.41113800 & -6.17482000 & -2.02744400 \\
\hline $\mathrm{C}$ & -2.95958400 & 3.01228900 & -1.49275600 & $\mathrm{C}$ & -3.57810300 & -4.21748100 & 0.14948600 \\
\hline $\mathrm{C}$ & 3.15725000 & 0.68921400 & 2.51345300 & $\mathrm{H}$ & -3.82713700 & -4.91030200 & -0.65758500 \\
\hline $\mathrm{H}$ & 3.24298600 & -0.15356200 & 1.81463800 & $\mathrm{C}$ & 1.85307900 & 0.49626300 & 3.28778300 \\
\hline $\mathrm{C}$ & -1.88897100 & 3.64420100 & 0.64115900 & $\mathrm{H}$ & 1.71454600 & 1.28461300 & 4.04353000 \\
\hline $\mathrm{C}$ & -3.64992800 & -2.83891400 & -0.07647000 & $\mathrm{H}$ & 1.85227800 & -0.47098300 & 3.81109100 \\
\hline $\mathrm{C}$ & 3.02301100 & 3.19224400 & 2.39190800 & $\mathrm{H}$ & 0.98675000 & 0.52435900 & 2.61230200 \\
\hline $\mathrm{H}$ & 2.93607500 & 3.18998400 & 3.48047000 & $\mathrm{C}$ & 0.21434900 & 1.77358400 & -1.77560800 \\
\hline $\mathrm{C}$ & -1.73740400 & 3.42724400 & 2.13329500 & $\mathrm{H}$ & 1.25663200 & 2.00657600 & -2.02211000 \\
\hline $\mathrm{H}$ & -1.89554300 & 2.35233100 & 2.31884500 & $\mathrm{H}$ & -0.40174500 & 2.66122800 & -1.95960400 \\
\hline $\mathrm{C}$ & 2.48736000 & -5.01115900 & -0.56436900 & $\mathrm{H}$ & -0.12342900 & 0.94047300 & -2.40180500 \\
\hline $\mathrm{H}$ & 2.65295600 & -5.86753700 & 0.09083800 & $\mathrm{C}$ & -1.07876000 & -1.65202000 & 3.72764200 \\
\hline $\mathrm{C}$ & -2.56436500 & -1.52522000 & 3.38377200 & $\mathrm{H}$ & -0.83679600 & -2.65675500 & 4.10863100 \\
\hline $\mathrm{H}$ & -2.72874100 & -0.49867900 & 3.02353100 & $\mathrm{H}$ & -0.79433900 & -0.92283000 & 4.50100900 \\
\hline $\mathrm{C}$ & -4.48984300 & 1.50874600 & 1.06409500 & $\mathrm{H}$ & -0.45274500 & -1.46345400 & 2.84231700 \\
\hline $\mathrm{H}$ & -5.19701400 & 2.15089000 & 0.52087400 & $\mathrm{C}$ & -4.07364500 & -2.30229400 & -1.42932700 \\
\hline $\mathrm{H}$ & -4.45341300 & 1.85007300 & 2.11313800 & $\mathrm{H}$ & -4.02799900 & -1.20489100 & -1.37460200 \\
\hline $\mathrm{C}$ & -1.36186000 & 4.77757500 & 0.01224600 & $\mathrm{C}$ & -4.82570700 & 0.02421700 & 0.96613000 \\
\hline $\mathrm{H}$ & -0.74633700 & 5.47352500 & 0.58427500 & $\mathrm{H}$ & -5.32419700 & -0.36241700 & 1.86590400 \\
\hline $\mathrm{C}$ & 1.86741900 & -1.68643600 & -3.27366800 & $\mathrm{H}$ & -5.46602000 & -0.20650100 & 0.09547100 \\
\hline $\mathrm{H}$ & 2.19127000 & -0.75907100 & -2.77918000 & $\mathrm{C}$ & 4.37487300 & 0.63197200 & 3.43995000 \\
\hline $\mathrm{C}$ & -2.86953100 & -3.84804500 & 2.42956100 & $\Pi$ & 5.31429300 & 0.76060700 & 2.88106600 \\
\hline $\mathrm{H}$ & -2.56548500 & -4.25377200 & 3.39713100 & $\mathrm{H}$ & 4.41682000 & -0.33671000 & 3.96183000 \\
\hline $\mathrm{C}$ & 1.56053200 & -4.10438400 & -2.60362100 & $\mathrm{H}$ & 4.33770500 & 1.42059500 & 4.20711500 \\
\hline
\end{tabular}




\begin{tabular}{|c|c|c|c|c|c|c|c|}
\hline $\mathrm{C}$ & 4.43362600 & -4.71934200 & 1.65085000 & $\mathrm{C}$ & -3.10720000 & -2.73061000 & -2.53401000 \\
\hline $\mathrm{H}$ & 3.77439800 & -5.55607900 & 1.92970800 & $\mathrm{H}$ & -2.09250100 & -2.35820900 & -2.33802900 \\
\hline $\mathrm{H}$ & 5.00121200 & -4.44503900 & 2.55248200 & $\mathrm{H}$ & -3.42520600 & -2.32480000 & -3.50601100 \\
\hline $\mathrm{H}$ & 5.14728500 & -5.08627200 & 0.89803900 & $\mathrm{H}$ & -3.05844300 & -3.82634000 & -2.63198200 \\
\hline C & 2.60928800 & -3.05158900 & 2.17151000 & $\mathrm{C}$ & -5.51557600 & -2.69845400 & -1.75705200 \\
\hline $\mathrm{H}$ & 2.07136600 & -2.15613300 & 1.82847100 & $\mathrm{H}$ & -5.61586000 & -3.78874100 & -1.87423900 \\
\hline $\mathrm{H}$ & 3.09474200 & -2.80616000 & 3.12829000 & $\mathrm{H}$ & -5.84147600 & -2.23170500 & -2.69921900 \\
\hline $\mathrm{H}$ & 1.86612900 & -3.84146000 & 2.36435500 & $\mathrm{H}$ & -6.21009000 & -2.38748200 & -0.96215200 \\
\hline $\mathrm{C}$ & -0.35497600 & 3.78530300 & 2.66451500 & & & & \\
\hline $\mathrm{H}$ & 0.42578300 & 3.25724200 & 2.10406600 & \multicolumn{4}{|c|}{$\operatorname{trans} \mathbf{- 3 \mathbf { a } ^ { \bullet + }}$} \\
\hline $\mathrm{H}$ & -0.27153600 & 3.50532500 & 3.72561000 & $\mathrm{P}$ & 0.00819700 & -1.28047400 & -0.02152200 \\
\hline $\mathrm{H}$ & -0.15571700 & 4.86622500 & 2.59928700 & $\mathrm{P}$ & 0.04607700 & 1.36334300 & 0.20874800 \\
\hline C & 4.98821500 & 3.63590300 & -2.18347100 & $\mathrm{~N}$ & 3.29339600 & -1.20910100 & -0.44083900 \\
\hline $\mathrm{H}$ & 5.22477200 & 4.64932000 & -1.82361300 & $\mathrm{~N}$ & -3.35265400 & 1.14139600 & 0.59578400 \\
\hline $\mathrm{H}$ & 5.19426300 & 3.60521700 & -3.26459200 & $\mathrm{~N}$ & 3.49926400 & 0.94969300 & 0.04486700 \\
\hline $\mathrm{H}$ & 5.68303000 & 2.94641400 & -1.68300700 & $\mathrm{~N}$ & -3.30887700 & -1.06132300 & 0.76483300 \\
\hline $\mathrm{C}$ & 2.60760300 & 4.29264300 & -2.61610100 & $\mathrm{C}$ & -2.54713100 & 0.04230300 & 0.49861000 \\
\hline $\boldsymbol{H}$ & 1.55047400 & 4.15348900 & -2.35262900 & $\mathrm{C}$ & 2.59789800 & -0.05208700 & -0.16569500 \\
\hline $\mathrm{H}$ & 2.70800200 & 4.18461400 & -3.70672600 & $\mathrm{C}$ & 3.16773100 & 2.25763700 & 0.48991200 \\
\hline $\mathrm{H}$ & 2.87880500 & 5.33078200 & -2.37063500 & $\mathrm{C}$ & 2.73483600 & -3.58805000 & -0.59972200 \\
\hline $\mathrm{C}$ & -4.77224000 & 2.77105900 & -3.25241300 & $\mathrm{C}$ & 2.73972500 & -2.30141700 & -1.17984300 \\
\hline $\mathrm{H}$ & -5.36214300 & 3.54196100 & -2.73444600 & $\mathrm{C}$ & 2.69966000 & 2.45230000 & 1.80659300 \\
\hline $\mathrm{H}$ & -5.47121700 & 2.05038000 & -3.70305600 & $\mathrm{C}$ & -2.84598000 & -2.40248600 & 0.84727600 \\
\hline $\mathrm{H}$ & -4.23432000 & 3.25737800 & -4.08066400 & $\mathrm{C}$ & 1.22140500 & 0.05113200 & -0.09743800 \\
\hline $\mathrm{C}$ & -3.45388000 & -1.73761300 & 4.61028700 & $\mathrm{C}$ & 4.71760000 & -0.90624200 & -0.59943900 \\
\hline $\mathrm{H}$ & -4.51991300 & -1.63478400 & 4.35704600 & $\mathrm{H}$ & 4.96650900 & -0.81889300 & -1.67204000 \\
\hline $\mathrm{H}$ & -3.21572000 & -1.00034200 & 5.39211800 & $\mathrm{H}$ & 5.34286200 & -1.69611900 & -0.16618900 \\
\hline $\mathrm{H}$ & -3.31127800 & -2.73858700 & 5.04633600 & $\mathrm{C}$ & 3.34518900 & 3.32823300 & -0.41092900 \\
\hline $\mathrm{C}$ & 2.75484800 & -1.89801300 & -4.50517600 & $\mathrm{C}$ & 2.27274200 & -2.08277900 & -2.49520200 \\
\hline $\mathrm{H}$ & 3.81089200 & -2.03026400 & -4.22625700 & $\mathrm{C}$ & -2.76892300 & -3.01189800 & 2.11650000 \\
\hline $\mathrm{H}$ & 2.68539400 & -1.03609500 & -5.18648000 & $\mathrm{C}$ & -1.19387600 & 0.05817800 & 0.19362700 \\
\hline $\mathrm{H}$ & 2.44903200 & -2.79497800 & -5.06588100 & $\mathrm{C}$ & 4.85587100 & 0.42204700 & 0.12681100 \\
\hline $\mathrm{C}$ & 0.40543000 & -1.49012600 & -3.67195700 & $\mathrm{H}$ & 5.15166700 & 0.28774200 & 1.18217500 \\
\hline $\mathrm{H}$ & 0.00378400 & -2.36243800 & -4.20967400 & $\mathrm{H}$ & 5.57660000 & 1.10020700 & -0.34788600 \\
\hline $\mathrm{H}$ & 0.29874200 & -0.61803500 & -4.33431500 & $\mathrm{C}$ & -3.10601800 & 2.40680600 & 0.00012900 \\
\hline $\mathrm{H}$ & -0.22083900 & -1.31779700 & -2.78431800 & $\mathrm{C}$ & 3.37589200 & -3.86527800 & 0.74613900 \\
\hline $\mathrm{C}$ & -2.96451700 & 1.02150200 & -3.03700800 & $\mathrm{H}$ & 3.45252200 & -2.89958200 & 1.27318900 \\
\hline $\mathrm{H}$ & -2.29666500 & 1.51326100 & -3.76121200 & $\mathrm{C}$ & -3.37310200 & 2.56737200 & -1.37590900 \\
\hline $\mathrm{H}$ & -3.60458300 & 0.31837300 & -3.59136100 & $\mathrm{C}$ & 2.64613500 & 1.31567100 & 2.81132000 \\
\hline $\mathrm{H}$ & -2.34135700 & 0.44432000 & -2.33997300 & $\mathrm{H}$ & 2.70864400 & 0.37006800 & 2.25317000 \\
\hline $\mathrm{C}$ & -2.82882200 & 4.20987300 & 2.87598000 & $\mathrm{C}$ & -2.67623900 & 3.47597200 & 0.80904200 \\
\hline П & -2.68316100 & 5.29352300 & 2.74421700 & $\mathrm{C}$ & -2.50094700 & -3.09219900 & -0.33701100 \\
\hline $\mathrm{H}$ & -2.79729400 & 3.99406600 & 3.95524400 & $\mathrm{C}$ & 2.37242600 & 3.75595900 & 2.19298900 \\
\hline $\mathrm{H}$ & -3.83652900 & 3.97053700 & 2.50626400 & $\mathrm{H}$ & 2.01495700 & 3.94116600 & 3.20738700 \\
\hline
\end{tabular}




\begin{tabular}{|c|c|c|c|c|c|c|c|}
\hline $\mathrm{C}$ & -2.44586700 & 3.28952300 & 2.29402000 & $\mathrm{H}$ & 0.97295400 & 3.06712600 & -1.16058600 \\
\hline $\mathrm{H}$ & -2.36255900 & 2.20478400 & 2.46957400 & $\mathrm{H}$ & -0.79653400 & 3.21530100 & -1.13168300 \\
\hline $\mathrm{C}$ & 2.18858300 & -4.64228600 & -1.33955900 & $\mathrm{H}$ & -0.02591000 & 1.96672300 & -2.17345000 \\
\hline $\mathrm{H}$ & 2.16366800 & -5.64375700 & -0.90670200 & $\mathrm{C}$ & -2.07016100 & -2.27515500 & 4.44083600 \\
\hline $\mathrm{C}$ & -3.17553100 & -2.28282800 & 3.38292800 & $\mathrm{H}$ & -1.82286100 & -3.29273900 & 4.78021700 \\
\hline $\mathrm{H}$ & -3.36394600 & -1.23323200 & 3.10995700 & $\mathrm{H}$ & -2.39328400 & -1.70460600 & 5.32482600 \\
\hline $\mathrm{C}$ & -4.67327700 & 0.78663100 & 1.10965900 & $\mathrm{H}$ & -1.14926200 & -1.81495500 & 4.05797700 \\
\hline $\mathrm{H}$ & -5.45469300 & 1.33709500 & 0.56855300 & $\mathrm{C}$ & -2.75623700 & -2.48111400 & -1.70196700 \\
\hline $\mathrm{H}$ & -4.75432500 & 1.03718000 & 2.18161600 & $\mathrm{H}$ & -2.68164700 & -1.38998500 & -1.59265900 \\
\hline 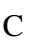 & -2.50823200 & 4.72785200 & 0.20670900 & $\mathrm{C}$ & -4.72502000 & -0.71737900 & 0.86821800 \\
\hline $\mathrm{H}$ & -2.17612600 & 5.57561300 & 0.80870600 & $\mathrm{H}$ & -5.20130800 & -1.26723000 & 1.68953600 \\
\hline $\mathrm{C}$ & 2.33156700 & -0.72959900 & -3.18050500 & $\mathrm{H}$ & -5.25542400 & -0.97270100 & -0.06504100 \\
\hline $\mathrm{H}$ & 2.70815500 & 0.01111600 & -2.46228200 & $\mathrm{C}$ & 3.86153700 & 1.39145200 & 3.74364100 \\
\hline $\mathrm{C}$ & -2.31394800 & -4.33325100 & 2.18317700 & $\mathrm{H}$ & 4.80693900 & 1.40226000 & 3.18099100 \\
\hline $\mathrm{H}$ & -2.24867100 & -4.83218500 & 3.15246000 & $\mathrm{H}$ & 3.88004600 & 0.53145900 & 4.43093700 \\
\hline $\mathrm{C}$ & 1.75172100 & -3.17285500 & -3.19677600 & $\mathrm{H}$ & 3.83294700 & 2.30908000 & 4.35170200 \\
\hline $\mathrm{H}$ & 1.38070700 & -3.02712000 & -4.21343700 & $\mathrm{C}$ & 4.79837000 & -4.40900500 & 0.54940400 \\
\hline $\mathrm{C}$ & -3.18694800 & 3.83589600 & -1.93377100 & $\mathrm{H}$ & 4.76866000 & -5.39203200 & 0.05418400 \\
\hline $\mathrm{H}$ & -3.37894000 & 3.99051700 & -2.99765500 & $\mathrm{H}$ & 5.30733900 & -4.53369600 & 1.51766200 \\
\hline $\mathrm{C}$ & 2.50895100 & 4.82277600 & 1.30878600 & $\mathrm{H}$ & 5.41401600 & -3.75064400 & -0.07997300 \\
\hline$H$ & 2.24407800 & 5.83227200 & 1.63145400 & $\mathrm{C}$ & 2.57001700 & -4.83210900 & 1.61688500 \\
\hline $\mathrm{C}$ & 3.00152600 & 4.61254200 & 0.02362100 & $\mathrm{H}$ & 1.51377000 & -4.54158600 & 1.69215500 \\
\hline $\mathrm{H}$ & 3.12585400 & 5.46161200 & -0.65190300 & $\mathrm{H}$ & 2.99021600 & -4.86878000 & 2.63325000 \\
\hline $\mathrm{C}$ & -1.91562400 & -5.01049300 & 1.03379900 & $\mathrm{H}$ & 2.60729900 & -5.85751800 & 1.21856000 \\
\hline $\mathrm{H}$ & -1.53898600 & -6.03327400 & 1.10808100 & $\mathrm{C}$ & -1.14365800 & 3.93273200 & 2.76351400 \\
\hline $\mathrm{C}$ & 3.95278200 & 3.11348000 & -1.78535300 & $\mathrm{H}$ & -0.29327100 & 3.57380600 & 2.16700700 \\
\hline $\mathrm{H}$ & 3.98066300 & 2.02656300 & -1.95865000 & $\mathrm{H}$ & -0.95266200 & 3.68335000 & 3.81801600 \\
\hline $\mathrm{C}$ & -3.83163600 & 1.41172500 & -2.24382200 & $\mathrm{H}$ & -1.17673500 & 5.03067700 & 2.68923400 \\
\hline $\mathrm{H}$ & -4.07564900 & 0.57163100 & -1.57753300 & $\mathrm{C}$ & 5.39402600 & 3.63757300 & -1.80736000 \\
\hline $\mathrm{C}$ & -2.76187500 & 4.90698700 & -1.15070000 & $\mathrm{H}$ & 5.41490800 & 4.73182400 & -1.68521900 \\
\hline $\mathrm{H}$ & -2.62600200 & 5.89149100 & -1.60424600 & $\mathrm{H}$ & 5.88378100 & 3.39703000 & -2.76351400 \\
\hline $\mathrm{C}$ & 0.37794700 & -2.02174500 & 1.62652300 & $\mathrm{H}$ & 5.99865900 & 3.20972300 & -0.99349600 \\
\hline $\mathrm{H}$ & -0.22112200 & -2.92125800 & 1.80322700 & $\mathrm{C}$ & 3.13631200 & 3.73029400 & -2.92176200 \\
\hline 11 & 0.19821600 & -1.27836500 & 2.41367100 & $\mathrm{H}$ & 2.11937700 & 3.31714800 & -2.96505200 \\
\hline $\mathrm{H}$ & 1.44153600 & -2.28840200 & 1.62385200 & $\mathrm{H}$ & 3.62212800 & 3.52774100 & -3.88831900 \\
\hline $\mathrm{C}$ & 1.69561400 & -4.43881000 & -2.62329700 & $\mathrm{H}$ & 3.05426800 & 4.82360100 & -2.82218300 \\
\hline $\mathrm{H}$ & 1.27625300 & -5.27520600 & -3.18709300 & $\mathrm{C}$ & -5.09916100 & 1.74728700 & -3.03096000 \\
\hline $\mathrm{C}$ & -2.00618000 & -4.39470900 & -0.21205500 & $\mathrm{H}$ & -5.90353100 & 2.10012600 & -2.36801000 \\
\hline $\mathrm{H}$ & -1.70293800 & -4.94162500 & -1.10548800 & $\mathrm{H}$ & -5.46288000 & 0.85770800 & -3.56743400 \\
\hline $\mathrm{C}$ & 1.34481400 & 1.27300100 & 3.61114500 & $\mathrm{H}$ & -4.91888200 & 2.53091600 & -3.78270800 \\
\hline $\mathrm{H}$ & 1.24222400 & 2.14596000 & 4.27353100 & $\mathrm{C}$ & -4.47134400 & -2.87218000 & 3.95157500 \\
\hline$\Pi$ & 1.32223400 & 0.37693800 & 4.24975800 & $\mathrm{H}$ & -5.27867900 & -2.88908600 & 3.20364500 \\
\hline $\mathrm{H}$ & 0.47117700 & 1.24849100 & 2.94418400 & $\mathrm{H}$ & -4.81837200 & -2.29003500 & 4.81904400 \\
\hline $\mathrm{C}$ & 0.03212300 & 2.50590100 & -1.22130600 & $\mathrm{H}$ & -4.31809000 & -3.91021300 & 4.28554200 \\
\hline
\end{tabular}




\begin{tabular}{|c|c|c|c|c|c|c|c|}
\hline C & 3.30572000 & -0.75004600 & -4.36189100 & $\mathrm{C}$ & 3.08627500 & -2.36207100 & -2.45441500 \\
\hline $\mathrm{H}$ & 4.31209400 & -1.06826900 & -4.05005000 & $\mathrm{C}$ & -2.47945300 & -2.79566600 & 2.25649400 \\
\hline $\mathrm{H}$ & 3.38704700 & 0.25148300 & -4.81225000 & $\mathrm{C}$ & -1.25834400 & 0.05633000 & -0.03995200 \\
\hline $\mathrm{H}$ & 2.97064900 & -1.44359900 & -5.14840300 & $\mathrm{C}$ & 4.83468400 & 0.41525000 & 0.00794100 \\
\hline C & 0.94006500 & -0.26197100 & -3.60685100 & $\mathrm{H}$ & 5.44671800 & 0.92390600 & 0.77911400 \\
\hline $\mathrm{H}$ & 0.51644100 & -0.90911200 & -4.38961500 & $\mathrm{H}$ & 5.31794000 & 0.58961300 & -0.98222000 \\
\hline $\mathrm{H}$ & 0.98190000 & 0.76097300 & -4.01111000 & $\mathrm{C}$ & -3.10113400 & 2.53888500 & -0.21920700 \\
\hline F & 0.24855400 & -0.27286300 & -2.75347900 & $\mathrm{C}$ & 2.76014300 & -3.85598300 & 1.11672000 \\
\hline C & -2.70275800 & 0.93564600 & -3.16080800 & $\mathrm{H}$ & 2.67276600 & -2.82520800 & 1.52041900 \\
\hline $\mathrm{H}$ & -2.36889000 & 1.74032600 & -3.83435400 & $\mathrm{C}$ & -3.54917900 & 2.53207200 & -1.57303900 \\
\hline $\mathrm{H}$ & -3.03748200 & 0.09242600 & -3.78361800 & $\mathrm{C}$ & 2.56995000 & 1.20258700 & 2.85784900 \\
\hline H & -1.83844900 & 0.60037100 & -2.57196600 & $\mathrm{H}$ & 2.58909200 & 0.28023300 & 2.24520400 \\
\hline C & -3.64639000 & 3.81164000 & 3.09110400 & $\mathrm{C}$ & -2.58623800 & 3.72766100 & 0.37265100 \\
\hline $\mathrm{H}$ & -3.75909700 & 4.89938700 & 2.95991300 & $\mathrm{C}$ & -3.52044500 & -3.09490300 & 0.02577800 \\
\hline $\mathrm{H}$ & -3.51787900 & 3.61345800 & 4.16645400 & $\mathrm{C}$ & 2.69829900 & 3.69331200 & 2.43417500 \\
\hline $\mathrm{H}$ & -4.58765300 & 3.34392300 & 2.76644000 & $\mathrm{H}$ & 2.40450500 & 3.83657300 & 3.48566600 \\
\hline C & -1.73959700 & -2.89796500 & -2.75814900 & $\mathrm{C}$ & -2.20796900 & 3.78235900 & 1.84854500 \\
\hline H & -0.71395500 & -2.72436700 & -2.40745200 & $\mathrm{H}$ & -2.14458300 & 2.72944600 & 2.19348600 \\
\hline $\mathrm{H}$ & -1.89288600 & -2.31696900 & -3.67957700 & $\mathrm{C}$ & 2.60418300 & -4.85732900 & -1.21377700 \\
\hline $\mathrm{H}$ & -1.83655300 & -3.96127500 & -3.02746700 & $\mathrm{H}$ & 2.41851600 & -5.83354500 & -0.74013000 \\
\hline C & -4.18162500 & -2.81255700 & -2.16141200 & $\mathrm{C}$ & -2.00294000 & -1.92498300 & 3.41696100 \\
\hline $\mathrm{H}$ & -4.29667800 & -3.89625000 & -2.31917900 & $\mathrm{H}$ & -2.04363100 & -0.86946400 & 3.07787300 \\
\hline $\mathrm{H}$ & -4.41019800 & -2.30583800 & -3.11188600 & $\mathrm{C}$ & -4.58920200 & 1.12009100 & 1.17386400 \\
\hline \multirow[t]{2}{*}{$\mathrm{H}$} & \multirow[t]{2}{*}{-4.93652700} & \multirow[t]{2}{*}{-2.50883800} & \multirow[t]{2}{*}{-1.42251400} & $\mathrm{H}$ & -5.37728400 & 1.09795800 & 0.38328200 \\
\hline & & & & $\mathrm{H}$ & -4.87140300 & 1.90344600 & 1.90690100 \\
\hline \multirow{2}{*}{\multicolumn{4}{|c|}{ cis-3a with PBE }} & $\mathrm{C}$ & -2.47723200 & 4.88267900 & -0.42530300 \\
\hline & & & & $\mathrm{H}$ & -2.07066600 & 5.80561100 & 0.01557300 \\
\hline \multirow{2}{*}{$\mathrm{P}$} & 0.01053800 & \multirow{2}{*}{1.38338300} & -0.0 & $\mathrm{C}$ & 3.42638400 & -1.05416500 & -3.16575600 \\
\hline & 3.29222700 & & & $\mathrm{H}$ & 3.31757400 & -0.23445200 & -2.42934400 \\
\hline $\mathrm{N}$ & -3.26516400 & 1.37371100 & 0.60387700 & $\mathrm{C}$ & -2.33860400 & -4.19459800 & 2.34558000 \\
\hline $\mathrm{N}$ & 3.45378200 & 0.91417400 & 0.04006900 & $\mathrm{H}$ & -1.88025600 & -4.63417800 & 3.24488800 \\
\hline $\mathrm{N}$ & -3.35490000 & -0.84277300 & 0.99783500 & C & 2.88348200 & -3.52733200 & -3.21833000 \\
\hline C & -2.53058300 & 0.17402100 & 0.47322900 & $\mathrm{H}$ & 2.92470000 & -3.46494200 & -4.31679200 \\
\hline C & 2.53591500 & -0.13422300 & -0.17167800 & $\mathrm{C}$ & -3.41269300 & 3.71313900 & -2.32908100 \\
\hline C & 3.19658900 & 2.22140300 & 0.56365000 & $\mathrm{H}$ & -3.74489400 & 3.72189700 & -3.37931500 \\
\hline $\mathrm{C}$ & 2.78759500 & -3.72035400 & -0.40239200 & $r$ & 2.96101800 & 4.81331400 & 1.63704100 \\
\hline C & 3.02379500 & -2.46511700 & -1.03240300 & $\mathrm{H}$ & 2.87084400 & 5.82596600 & 2.05972800 \\
\hline C & 2.81129600 & 2.38593900 & 1.92491900 & $\mathrm{C}$ & 3.33751900 & 4.64002300 & 0.30105800 \\
\hline $\mathrm{C}$ & -3.07748600 & -2.24713600 & 1.08730800 & $\mathrm{H}$ & 3.54243400 & 5.52372800 & -0.32273300 \\
\hline C & 1.17019400 & -0.06087600 & -0.31245000 & $\mathrm{C}$ & -2.75332500 & -5.03302700 & 1.30658400 \\
\hline $\mathrm{C}$ & 4.65069800 & -1.07927900 & 0.28430500 & $\mathrm{H}$ & -2.62480200 & -6.12328700 & 1.39170600 \\
\hline $\mathrm{H}$ & 5.38238400 & -1.71260600 & -0.25416500 & $\mathrm{C}$ & 3.91088900 & 3.20662200 & -1.71174800 \\
\hline $\mathrm{H}$ & 4.73674200 & -1.30414700 & 1.37387100 & $\mathrm{H}$ & 3.70111300 & 2.15540700 & -2.00360100 \\
\hline & 3.46136000 & 3.35440100 & -0.26010300 & $\mathrm{C}$ & -4.21866500 & 1.31731700 & -2.21307600 \\
\hline
\end{tabular}




\begin{tabular}{|c|c|c|c|}
\hline & -4.19833400 & 0.49571500 & -1.47067200 \\
\hline & -2.87484100 & 4.87711700 & -1.76749500 \\
\hline & -2.77589600 & .78908800 & -2.37671100 \\
\hline & -0.41616300 & -2.13267200 & .75149600 \\
\hline $\mathrm{H}$ & -0.54552000 & 9900 & 7300 \\
\hline $\mathrm{H}$ & -1.34343400 & -2.73 & -1.67109200 \\
\hline I & 0.41070400 & -2.81613000 & -2.02492500 \\
\hline $\mathrm{C}$ & 2.64520100 & -4.76597900 & -2.60943800 \\
\hline $\mathrm{H}$ & 2.49432700 & -5.66483500 & -3.22725500 \\
\hline $\mathrm{C}$ & -3.33294900 & -4.48368500 & 0.15585100 \\
\hline $\mathrm{H}$ & -3.65745600 & 76300 & 200 \\
\hline $\mathrm{C}$ & 1.18285200 & 1.26531400 & 16700 \\
\hline $\mathrm{H}$ & 1.07828000 & 2.15204300 & 4.17919300 \\
\hline $\mathrm{H}$ & 1.01330000 & 0.36538300 & 4.14406200 \\
\hline $\mathrm{H}$ & 0.38302200 & 1.30969600 & 1200 \\
\hline $\mathrm{C}$ & -0.04316600 & 1.7734 & 6400 \\
\hline $\mathrm{H}$ & 0.93257800 & 2.22667500 & -2.43225700 \\
\hline $\mathrm{H}$ & -0.8 & 2.5 & 300 \\
\hline $\mathrm{H}$ & -0.19580700 & 0.87191900 & -2.78926100 \\
\hline $\mathrm{C}$ & -0.54036900 & -2.21215000 & 3.79847700 \\
\hline $\mathrm{H}$ & -0.40 & 00 & 00 \\
\hline $\mathrm{H}$ & -0.21264400 & -1.52183900 & 4.60265500 \\
\hline $\mathrm{H}$ & 0.12621600 & -2.07976200 & 5000 \\
\hline $\mathrm{C}$ & -4.22796500 & -2.52237800 & -1.20249700 \\
\hline$\Pi$ & -3.76989400 & -1.53090400 & -1.39941000 \\
\hline $\mathrm{C}$ & -4.40764000 & -0.24923700 & 1.82279900 \\
\hline $\mathrm{H}$ & -4.09382400 & -0.14192100 & 2.88825400 \\
\hline $\mathrm{H}$ & -5.32800300 & -0.86652000 & 1.80370000 \\
\hline $\mathrm{C}$ & 3.69119500 & 1.08867800 & 3.90908600 \\
\hline $\mathrm{H}$ & 4.69028700 & 0.99699700 & 12800 \\
\hline $\mathrm{H}$ & 3.53638500 & 0.19818100 & 4.55339800 \\
\hline $\mathrm{H}$ & 3.71989200 & 98017300 & 4.57019000 \\
\hline $\mathrm{C}$ & 4.07471900 & -4.46944300 & 1.64015000 \\
\hline $\mathrm{H}$ & 4.20 & -5.5 & 1.26579900 \\
\hline $\mathrm{H}$ & 4.07618300 & -4.51055700 & 2.74941100 \\
\hline $\mathrm{H}$ & 4.96295900 & -3.88861500 & 1.31940300 \\
\hline $\mathrm{C}$ & 1.54436400 & -4.65090400 & 1.61906300 \\
\hline-1 & 0.59899500 & -4.19657300 & 1.26095000 \\
\hline & 1.52 & -4.656221 & 2.72839300 \\
\hline $\mathrm{H}$ & 1.57400500 & -5.71060300 & 1.28925000 \\
\hline 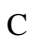 & -0.84010000 & 4.43692200 & 2.08866100 \\
\hline $\mathrm{H}$ & -0.05338500 & 3.93465400 & 1.49262800 \\
\hline $\mathrm{H}$ & -0.56183700 & 4.36292200 & 3.16032700 \\
\hline $\mathrm{H}$ & -0.84273300 & 5.51662100 & 1.82852000 \\
\hline
\end{tabular}

\begin{tabular}{|c|c|c|c|}
\hline$C$ & 5.42754700 & 3.45103600 & -1.85567400 \\
\hline & 5.68175600 & 4.50030200 & -1.59609900 \\
\hline & 5.76010900 & 3.27320700 & -2.89979600 \\
\hline & 6.02299200 & 2.79445300 & -1.19122700 \\
\hline & 3.13721400 & 4.12480400 & -2.67494300 \\
\hline & 2.04117700 & 4.01586200 & -2.55857300 \\
\hline & 3.39683100 & 3.88744300 & -3.72728800 \\
\hline & 3.38633700 & 5.19415300 & -2.51299500 \\
\hline C & -5.69647300 & 1.61269000 & -2.54027500 \\
\hline & -6.25514100 & 1.96072300 & -1.64755400 \\
\hline $\mathrm{H}$ & -6.20118700 & 0.70303400 & -2.92742200 \\
\hline & -5.78935100 & 2.40134200 & -3.31582300 \\
\hline C & -2.92521500 & -2.07986700 & 4.64334400 \\
\hline H & -3.98488900 & -1.86162400 & 4.40078300 \\
\hline $\mathrm{H}$ & -2.61242500 & -1.39674800 & 5.46026300 \\
\hline $\mathrm{H}$ & -2.88590900 & -3.11597600 & 5.04023400 \\
\hline C & 4.89188200 & -1.06224500 & -3.64622100 \\
\hline $\mathrm{H}$ & 5.59842600 & -1.25544500 & -2.81338300 \\
\hline $\mathrm{H}$ & 5.16272200 & -0.08805700 & -4.10483300 \\
\hline $\mathrm{F}$ & 5.05824700 & -1.85217400 & -4.40841300 \\
\hline C & 2.47044000 & -0.73340000 & -4.32676000 \\
\hline $\mathrm{H}$ & 2.56429400 & -1.46155900 & -5.15924500 \\
\hline $\mathrm{H}$ & 2.69447500 & 0.26984200 & -4.74439900 \\
\hline $\mathrm{H}$ & 1.41545100 & -0.73352700 & -3.98980800 \\
\hline $\mathrm{C}$ & -3.47496800 & 0.80885300 & -3.46030000 \\
\hline $\mathrm{H}$ & -3.44382300 & 1.57554300 & -4.26244300 \\
\hline $\mathrm{H}$ & -3.98543500 & -0.08437600 & -3.87678400 \\
\hline $\mathrm{H}$ & -2.43365200 & 0.52420900 & -3.21808800 \\
\hline C & -3.30846300 & 4.48813900 & 2.66725000 \\
\hline $\mathrm{H}$ & -3.41172400 & 5.55107900 & 2.36254500 \\
\hline $\mathrm{H}$ & -3.06435100 & 4.47144200 & 3.74998400 \\
\hline I & -4.30067100 & 4.01063300 & 2.53412600 \\
\hline C & -4.06423300 & -3.36732800 & -2.47567300 \\
\hline $\mathrm{H}$ & -3.00195100 & -3.59280300 & -2.69588100 \\
\hline $\mathrm{H}$ & -4.48006600 & -2.82160000 & -3.34715100 \\
\hline$\digamma$ & -4.61110400 & -4.33113000 & -2.41030100 \\
\hline $\mathrm{C}$ & -5.72852700 & -2.29807000 & -0.92296200 \\
\hline $\mathrm{H}$ & -6.23505700 & -3.26183900 & -0.70637100 \\
\hline $\mathrm{H}$ & -6.22846400 & -1.84230700 & -1.80311500 \\
\hline $\mathrm{H}$ & -5.89722500 & -1.62924400 & -0.05764700 \\
\hline
\end{tabular}

\section{trans-3a with PBE}

$\begin{array}{llll}\mathrm{P} & -0.01440100 & -1.38504000 & 0.13084800\end{array}$

$\begin{array}{llll}\mathrm{P} & -0.00859600 & 1.37651000 & -0.08218700\end{array}$ 


\begin{tabular}{|c|c|c|c|c|c|c|c|}
\hline $\mathrm{N}$ & 3.37079800 & -1.17548900 & -0.45398100 & $\mathrm{H}$ & 2.38307600 & -0.25606200 & -2.57900700 \\
\hline $\mathrm{N}$ & -3.48323600 & 1.11189100 & 0.09674600 & $\mathrm{C}$ & -2.76631200 & -4.33121100 & 2.37628200 \\
\hline $\mathrm{N}$ & 3.42646500 & 1.08015600 & -0.24850700 & $\mathrm{H}$ & -2.78514200 & -4.77552000 & 3.38359100 \\
\hline $\mathrm{N}$ & -3.43808400 & -1.05301600 & 0.69826200 & $\mathrm{C}$ & 2.14075100 & -3.61849100 & -3.02332200 \\
\hline $\mathrm{C}$ & -2.61175300 & 0.02154700 & 0.30520900 & $\mathrm{H}$ & 1.76537400 & -3.63016500 & -4.05759100 \\
\hline $\mathrm{C}$ & 2.56071200 & -0.03517500 & -0.26021800 & $\mathrm{C}$ & -3.13045700 & 4.35230700 & -1.74434800 \\
\hline $\mathrm{C}$ & 3.19330700 & 2.31981100 & 0.42806100 & $\mathrm{H}$ & -3.35639300 & 4.78283700 & -2.73230600 \\
\hline $\mathrm{C}$ & 3.12588700 & -3.62236500 & -0.38089300 & $\mathrm{C}$ & 2.84028200 & 4.79165900 & 1.73623500 \\
\hline $\mathrm{C}$ & 2.96808000 & -2.39401400 & -1.08850200 & $\mathrm{H}$ & 2.69751400 & 5.75567100 & 2.24865300 \\
\hline $\mathrm{C}$ & 2.99728900 & 2.34893900 & 1.84145700 & $\mathrm{C}$ & 3.06272200 & 4.75425000 & 0.35666700 \\
\hline $\mathrm{C}$ & -3.05398000 & -2.41181300 & 0.90333800 & $\mathrm{H}$ & 3.09472700 & 5.69614000 & -0.21191000 \\
\hline $\mathrm{C}$ & 1.19719000 & -0.01719800 & -0.11055600 & $\mathrm{C}$ & -2.40907300 & -5.12502600 & 1.28068500 \\
\hline $\mathrm{C}$ & 4.78899200 & -0.81905500 & -0.33639400 & $\mathrm{H}$ & -2.15351200 & -6.18599000 & 1.42729700 \\
\hline $\mathrm{H}$ & 5.39770600 & -1.40252500 & -1.05469000 & $\mathrm{C}$ & 3.49987400 & 3.54242500 & -1.82647000 \\
\hline $\mathrm{H}$ & 5.18408500 & -1.01490400 & 0.68831400 & $\mathrm{H}$ & 3.31128600 & 2.50943800 & -2.19025600 \\
\hline $\mathrm{C}$ & 3.23563100 & 3.53227800 & -0.32224900 & $\mathrm{C}$ & -4.03972400 & 2.15421400 & -2.61730100 \\
\hline $\mathrm{C}$ & 2.48883200 & -2.38921200 & -2.43304800 & $\mathrm{H}$ & -3.97545500 & 1.09286800 & -2.30137900 \\
\hline $\mathrm{C}$ & -3.07522700 & -2.96574400 & 2.21824900 & $\mathrm{C}$ & -2.53490500 & 5.15387600 & -0.76415200 \\
\hline $\mathrm{C}$ & -1.24089600 & 0.00843700 & 0.15691800 & $\mathrm{H}$ & -2.29935000 & 6.20752000 & -0.98049200 \\
\hline $\mathrm{C}$ & 4.78031000 & 0.68151000 & -0.64873000 & $\mathrm{C}$ & 0.29586800 & -1.82470200 & 1.93299800 \\
\hline $\mathrm{H}$ & 5.53876400 & 1.24361600 & -0.06907400 & $\mathrm{H}$ & -0.37980100 & -2.64714100 & 2.23795800 \\
\hline $\mathrm{H}$ & 4.96735300 & 0.86856900 & -1.73238500 & $\mathrm{H}$ & 0.15908100 & -0.94989500 & 2.59838500 \\
\hline $\mathrm{C}$ & -3.12828100 & 2.45955700 & -0.21023200 & $\mathrm{H}$ & 1.34103800 & -2.18172700 & 2.01869700 \\
\hline $\mathrm{C}$ & 3.68749200 & -3.66952600 & 1.03843400 & $\mathrm{C}$ & 2.28512700 & -4.82890000 & -2.33449800 \\
\hline $\mathrm{H}$ & 3.60673000 & -2.63917100 & 1.44673000 & $\mathrm{H}$ & 2.01341300 & -5.77819700 & -2.82161400 \\
\hline $\mathrm{C}$ & -3.42028500 & 2.99536400 & -1.50008100 & $\mathrm{C}$ & -2.37645900 & -4.56742600 & -0.00304800 \\
\hline $\mathrm{C}$ & 3.08258400 & 1.08949700 & 2.70173200 & $\mathrm{H}$ & -2.09407600 & -5.19964000 & -0.85674100 \\
\hline $\mathrm{H}$ & 2.87235200 & 0.22182500 & 2.04708200 & $\mathrm{C}$ & 2.03787300 & 1.05032800 & 3.82826500 \\
\hline $\mathrm{C}$ & -2.54334500 & 3.27499400 & 0.80597200 & $\mathrm{H}$ & 2.23257600 & 1.81418600 & 4.60993700 \\
\hline $\mathrm{C}$ & -2.71518900 & -3.21883300 & -0.22543400 & $\mathrm{H}$ & 2.05273100 & 0.06186000 & 4.33109500 \\
\hline $\mathrm{C}$ & 2.80833800 & 3.59648700 & 2.46485200 & $\mathrm{H}$ & 1.01692900 & 1.21959600 & 3.43231500 \\
\hline $\mathrm{H}$ & 2.65156700 & 3.63455100 & 3.55333000 & $\mathrm{C}$ & -0.08330100 & 1.82294200 & -1.90708000 \\
\hline $\mathrm{C}$ & -2.34764700 & 2.74718500 & 2.22693000 & $\mathrm{H}$ & 0.92692100 & 2.15941100 & 225600 \\
\hline $\mathrm{H}$ & -2.06678500 & 1.67688600 & 2.14327200 & $\mathrm{H}$ & -0.79119300 & 2.66065600 & -2.05884700 \\
\hline $\mathrm{C}$ & 2.78405000 & -4.82550600 & -1.02876900 & $\mathrm{H}$ & -0.37631600 & 0.95558300 & -2.53043400 \\
\hline $\mathrm{H}$ & 2.90344800 & -5.77938400 & -0.49271800 & $\mathrm{C}$ & -2.41052100 & -2.28340700 & 4.59939200 \\
\hline $\mathrm{C}$ & -3.42796400 & -2.13506100 & 3.45316100 & $\mathrm{H}$ & -2.39206000 & -3.31510200 & 5.00773600 \\
\hline $\mathrm{H}$ & -3.40750700 & -1.06915200 & 3.14690600 & $\mathrm{H}$ & -2.67982400 & -1.60745100 & 5.43715600 \\
\hline $\mathrm{C}$ & -4.88138600 & 0.68336900 & 0.13851600 & $\mathrm{H}$ & -1.38436600 & -2.02889000 & 4.27178900 \\
\hline $\mathrm{H}$ & -5.29219100 & 0.47380900 & -0.87689000 & $\mathrm{C}$ & -2.81800000 & -2.66996800 & -1.64821900 \\
\hline $\mathrm{H}$ & -5.51936000 & 1.45867600 & 0.60917400 & $\mathrm{H}$ & -2.50351800 & -1.60639700 & -1.61311800 \\
\hline $\mathrm{C}$ & -2.23788900 & 4.61344500 & 0.49274600 & $\mathrm{C}$ & -4.80206100 & -0.59618500 & 0.96498800 \\
\hline $\mathrm{H}$ & -1.76943700 & 5.25169000 & 1.25524700 & $\mathrm{H}$ & -4.97396500 & -0.38005600 & 2.04551100 \\
\hline $\mathrm{C}$ & 2.43478400 & -1.11564500 & -3.27480600 & $\mathrm{H}$ & -5.54349100 & -1.35758500 & 0.64868500 \\
\hline
\end{tabular}




\begin{tabular}{|c|c|c|c|c|c|c|c|}
\hline E & 4.50764800 & 0.92394900 & 3.26821600 & $\mathrm{C}$ & -3.67208400 & 9100 & 300 \\
\hline $\mathrm{H}$ & 5.27192800 & 0.90254300 & 2.46470900 & $\mathrm{H}$ & -3.99703400 & 3.88256400 & 3.13568000 \\
\hline H & 4.59573300 & -0.01918000 & 3.84747200 & $\mathrm{H}$ & -3.55212600 & 2.39174100 & 4.02855400 \\
\hline & 4.76668800 & 1.76407100 & 3.94641900 & $\mathrm{H}$ & -4.49203700 & 2.28216000 & 2.50689700 \\
\hline & 5.17908600 & -4.06614500 & 1.03515700 & $\mathrm{C}$ & -1.89609500 & -3.37473800 & -2.65261200 \\
\hline $\mathrm{H}$ & 5.30744900 & -5.09649900 & 0.64191700 & $\mathrm{H}$ & -0.84842600 & -3.38408200 & -2.29155300 \\
\hline & 5.59712300 & -4.04392300 & 2.06325500 & $\mathrm{H}$ & -1.92124700 & -2.84369900 & -3.62624800 \\
\hline & 5.79212300 & -3.39437300 & 0.40306300 & $\mathrm{H}$ & -2.21232600 & -4.42068500 & -2.85166200 \\
\hline & 2.89990100 & -4.60898500 & 1.96984900 & $\mathrm{C}$ & -4.28209600 & -2.71352700 & -2.13360000 \\
\hline $\mathrm{H}$ & 1.81303100 & -4.39873700 & 1.94798300 & $\mathrm{H}$ & -4.64693900 & -3.76018500 & -2.19830300 \\
\hline I & 3.25163400 & -4.49699100 & 3.01615100 & $\mathrm{H}$ & -4.37404000 & -2.25972300 & -3.14268900 \\
\hline & 3.04272500 & -5.67447700 & 1.69424800 & $\mathrm{H}$ & -4.96239000 & -2.16443600 & -1.45271700 \\
\hline & -1.21966000 & 3.44691600 & 2.99743500 & & & & \\
\hline $\mathrm{H}$ & -0.27418300 & 3.43095200 & 2.41953700 & \multicolumn{4}{|c|}{ 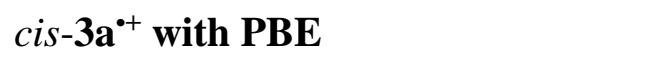 } \\
\hline $\mathrm{H}$ & -1.04334900 & 2.92954800 & 3.96270300 & $\mathrm{P}$ & 0.17784700 & 1.29269300 & 0.06810800 \\
\hline $\mathrm{H}$ & -1.46879700 & 4.50141800 & 3.24158300 & $\mathrm{P}$ & -0.14094400 & -1.37421700 & -0.07521300 \\
\hline C & 4.97140400 & 3.89518200 & -2.13063200 & $\mathrm{~N}$ & -3.29961500 & 1.48231800 & -0.15282700 \\
\hline $\mathrm{H}$ & 5.20170800 & 4.92786900 & -1.79412300 & $\mathrm{~N}$ & 3.24510700 & -1.56286000 & 0.26621700 \\
\hline $\mathrm{H}$ & 5.17414700 & 3.84339300 & -3.22078000 & $\mathrm{~N}$ & -3.49995800 & -0.72152400 & 0.20063500 \\
\hline $\mathrm{H}$ & 5.68151100 & 3.21683300 & -1.61869600 & $\mathrm{~N}$ & 3.51434100 & 0.61569400 & 0.69119500 \\
\hline $\mathrm{C}$ & 2.56638100 & 4.49172400 & -2.59972000 & $\mathrm{C}$ & 2.60464900 & -0.33612200 & 0.26787700 \\
\hline $\mathrm{H}$ & 1.50057200 & 4.31247200 & -2.35902600 & $\mathrm{C}$ & -2.58886500 & 0.29849200 & -0.03386700 \\
\hline $\mathrm{H}$ & 2.70008500 & 4.35632500 & -3.69278600 & $\mathrm{C}$ & -3.26468000 & -2.02775600 & 0.75587600 \\
\hline $\mathrm{H}$ & 2.78770000 & 5.55625200 & -2.37748700 & $\mathrm{C}$ & -2.67109200 & 3.85789600 & -0.08495000 \\
\hline $\mathrm{C}$ & -5.52581700 & 2.51511200 & -2.82186900 & $\mathrm{C}$ & -2.90611500 & 2.67585000 & -0.84132000 \\
\hline $\mathrm{H}$ & -6.11124900 & 2.42826900 & -1.88504100 & $\mathrm{C}$ & -2.88985600 & -2.14823300 & 2.12546300 \\
\hline $\mathrm{H}$ & -5.99378200 & 1.85565900 & -3.58214700 & $\mathrm{C}$ & 3.32129900 & 2.03132500 & 0.81539500 \\
\hline $\mathrm{H}$ & -5.62862600 & 3.56202100 & -3.17674400 & $\mathrm{C}$ & -1.19850600 & 0.16440900 & -0.13415400 \\
\hline $\mathrm{C}$ & -4.84618000 & -2.47204300 & 3.95901200 & $\mathrm{C}$ & -4.69896500 & 1.29050900 & 0.26332700 \\
\hline $\mathrm{H}$ & -5.61240900 & -2.35646200 & 3.16695700 & $\mathrm{H}$ & -5.37746100 & 1.88926300 & -0.37316800 \\
\hline $\mathrm{H}$ & -5.13019000 & -1.81872400 & 4.81014000 & $\mathrm{H}$ & -4.84446300 & 1.60847300 & 1.32082800 \\
\hline $\mathrm{H}$ & -4.89570000 & -3.52348800 & 4.31192100 & $\mathrm{C}$ & -3.54772300 & -3.17745600 & -0.03866200 \\
\hline $\mathrm{C}$ & 3.72173000 & -0.97256000 & -4.11303400 & $\mathrm{C}$ & -2.87330700 & 2.68210700 & -2.26690900 \\
\hline $\mathrm{H}$ & 4.63187700 & -0.98742000 & -3.47940500 & $\mathrm{C}$ & 2.73008200 & 2.57247400 & 1.99346000 \\
\hline $\mathrm{H}$ & 3.71868600 & -0.01945800 & -4.68263800 & $\mathrm{C}$ & 1.25701700 & -0.13331300 & -0.07745900 \\
\hline $\mathrm{H}$ & 3.81566700 & -1.80382000 & -4.84312600 & $\mathrm{C}$ & -4.88151400 & -0.21481300 & 0.08504700 \\
\hline $\mathrm{C}$ & 1.18816800 & -1.03011600 & -4.16974800 & $\mathrm{H}$ & -5.52163700 & -0.67077100 & 0.86389900 \\
\hline $\mathrm{H}$ & 1.20571700 & -1.77981000 & -4.98830000 & $\mathrm{H}$ & -5.31331500 & -0.46509400 & -0.90938700 \\
\hline $\mathrm{H}$ & 1.12630500 & -0.03106400 & -4.64742300 & $\mathrm{C}$ & 2.82877500 & -2.73787400 & -0.45274900 \\
\hline $\mathrm{H}$ & 0.26210500 & -1.18828300 & -3.58230000 & $\mathrm{C}$ & -2.74215400 & 3.86951000 & 1.43936000 \\
\hline $\mathrm{C}$ & -3.28185900 & 2.26270700 & -3.95332300 & $\mathrm{H}$ & -2.77207500 & 2.80946100 & 1.77039200 \\
\hline $\mathrm{H}$ & -3.33139200 & 3.28711200 & -4.37715000 & $\mathrm{C}$ & 3.06721000 & -2.81761300 & -1.85603100 \\
\hline$\Pi$ & -3.73168900 & 1.57908600 & -4.70274500 & $\mathrm{C}$ & -2.69120600 & -0.94123100 & 3.03951400 \\
\hline $\mathrm{H}$ & -2.21442700 & 1.99232300 & -3.84158200 & $\mathrm{H}$ & -2.63310300 & -0.03399900 & 2.40551200 \\
\hline
\end{tabular}




\begin{tabular}{|c|c|c|c|c|c|c|c|}
\hline C & 2.29796200 & -3.83674100 & 100 & $\mathrm{H}$ & -1.36 & -1.86338100 & 4.5 \\
\hline $\mathrm{C}$ & 3.83209500 & 2.87130500 & -0.21784800 & $\mathrm{H}$ & -1.23544400 & -0.08841500 & 4.42750900 \\
\hline$c$ & -2.76896400 & -3.44193900 & 2.66610300 & $\mathrm{H}$ & -0.50423800 & -1.12492600 & 3.15515100 \\
\hline H & -2.48281400 & -3.55885500 & 3.72219100 & $\mathrm{C}$ & -0.31706400 & -1.84366200 & -1.88248400 \\
\hline C & 2.18105600 & -3.80992200 & 1.80115100 & $\mathrm{H}$ & -1.34981800 & -2.21658000 & -2.01518200 \\
\hline $\mathrm{H}$ & 2.26918300 & -2.74791300 & 2.11295900 & $\mathrm{H}$ & 0.39001600 & -2.66107400 & -2.11975700 \\
\hline $\mathrm{C}$ & -2.38932300 & 5.04830000 & -0.78485800 & $\mathrm{H}$ & -0.15126200 & -0.98122000 & -2.55584900 \\
\hline $\mathrm{H}$ & -2.20781100 & 5.97645300 & -0.22203200 & $\mathrm{C}$ & 0.98861300 & 2.02882000 & 3.79500200 \\
\hline $\mathrm{C}$ & 2.36359800 & 1.70083100 & 3.19277500 & $\mathrm{H}$ & 0.95357100 & 3.05541500 & 4.21389200 \\
\hline $\mathrm{H}$ & 2.32700500 & 0.64739200 & 2.84358300 & $\mathrm{H}$ & 0.76330000 & 1.33229100 & 4.62715100 \\
\hline $\mathrm{C}$ & 4.66304200 & -1.42820400 & 0.63017400 & $\mathrm{H}$ & 0.18482600 & 1.93701100 & 3.03807200 \\
\hline $\mathrm{H}$ & 5.30659300 & -1.55066600 & -0.26939100 & $\mathrm{C}$ & 4.59599800 & 2.31544100 & -1.41853000 \\
\hline $\mathrm{H}$ & 4.95860600 & -2.20653500 & 1.36094800 & $\mathrm{H}$ & 4.50058000 & 1.21054800 & 11400 \\
\hline $\mathrm{C}$ & 1.94614700 & -4.99766200 & -0.43607800 & $\mathrm{C}$ & 4.73546100 & -0.01421700 & 1.21140400 \\
\hline $\mathrm{H}$ & 1.52409300 & -5.85759200 & 0.10502300 & $\mathrm{H}$ & 4.72220600 & -0.02105700 & 2.32422200 \\
\hline $\mathrm{C}$ & -3.21594700 & 1.45042800 & -3.10308600 & $\mathrm{H}$ & 5.63672100 & 0.54295700 & 0.88850000 \\
\hline $\mathrm{H}$ & -3.21238300 & 0.57030800 & -2.42914800 & $\mathrm{C}$ & -3.90038200 & -0.77051400 & 3.98170800 \\
\hline $\mathrm{C}$ & 2.58792400 & 3.97251600 & 2.07386200 & $\mathrm{H}$ & -4.85151900 & -0.67027100 & 3.41976100 \\
\hline $\mathrm{H}$ & 2.13091400 & 4.41738200 & 2.97046900 & $\mathrm{H}$ & -3.78125800 & 0.13247700 & 4.61534700 \\
\hline $\mathrm{C}$ & -2.57475000 & 3.89745600 & -2.91234200 & $\mathrm{H}$ & -4.00896300 & -1.64375800 & 4.65752900 \\
\hline $\mathrm{H}$ & -2.54352000 & 3.93116200 & -4.01199200 & $\mathrm{C}$ & -4.03134800 & 4.56032000 & 1.92837200 \\
\hline $\mathrm{C}$ & 2.70302600 & -4.00693300 & -2.51773400 & $\mathrm{H}$ & -4.04281900 & 5.63125300 & 1.63762000 \\
\hline $\mathrm{H}$ & 2.87302600 & -4.09515300 & -3.60189900 & $\mathrm{H}$ & -4.10762800 & 4.51266100 & 3.03391900 \\
\hline $\mathrm{C}$ & -3.02208600 & -4.58176800 & 1.89498800 & $\mathrm{H}$ & -4.94235200 & 4.09451400 & 1.50211500 \\
\hline $\mathrm{H}$ & -2.92635200 & -5.58269800 & 2.34254600 & $\mathrm{C}$ & -1.50028400 & 4.51363700 & 2.07994900 \\
\hline $\mathrm{C}$ & -3.41036900 & -4.44571700 & 0.55854100 & $\mathrm{H}$ & -0.56470500 & 4.03292300 & 1.72970100 \\
\hline $\mathrm{H}$ & -3.62310200 & -5.34669000 & -0.03580300 & $\mathrm{H}$ & -1.54204900 & 4.41428600 & 3.18373500 \\
\hline $\mathrm{C}$ & 3.04296200 & 4.80987000 & 1.04822800 & $\mathrm{H}$ & -1.43388600 & 5.59761200 & 1.85309300 \\
\hline $\mathrm{H}$ & 2.92649500 & 5.90077000 & 1.13757900 & $\mathrm{C}$ & 0.83011300 & -4.33270300 & 2.31249100 \\
\hline $\mathrm{C}$ & -4.05864600 & -3.08201100 & -1.47607800 & $\mathrm{H}$ & -0.01417800 & -3.79155800 & 1.84335800 \\
\hline $\mathrm{H}$ & -3.83196500 & -2.05541200 & -1.83877200 & $\mathrm{H}$ & 0.76053700 & -4.19782400 & 3.41132300 \\
\hline $\mathrm{C}$ & 3.74572400 & -1.70153800 & -2.65057600 & $\mathrm{H}$ & 0.70261000 & -5.41693800 & 2.11283200 \\
\hline $\mathrm{H}$ & 3.90508500 & -0.84410200 & -1.96541100 & $\mathrm{C}$ & -5.58924900 & -3.28449900 & -1.52801500 \\
\hline $\mathrm{C}$ & 2.13832600 & -5.08216500 & -1.82031000 & $\mathrm{H}$ & -5.85446900 & -4.31391400 & -1.21020500 \\
\hline $\mathrm{H}$ & 1.86086600 & -6.00120300 & -2.35891600 & $\mathrm{H}$ & -5.97032600 & -3.14345300 & -2.56027700 \\
\hline $\mathrm{C}$ & 0.45442000 & 2.53310900 & -1.28023700 & $\mathrm{H}$ & -6.13539600 & -2.58809500 & -0.86264400 \\
\hline $\mathrm{H}$ & 0.42370400 & 2.04076600 & -2.27072300 & $\mathrm{C}$ & -3.38001300 & -4.07919700 & -2.43397600 \\
\hline $\mathrm{H}$ & 1.44890400 & 2.98424100 & -1.10570000 & $\mathrm{H}$ & -2.27479300 & -4.03312800 & -2.38084900 \\
\hline $\mathrm{H}$ & -0.31263000 & 3.32585300 & -1.22149200 & $\mathrm{H}$ & -3.68292000 & -3.87178800 & -3.48024300 \\
\hline $\mathrm{C}$ & -2.33735600 & 5.07019900 & -2.18372400 & $\mathrm{H}$ & -3.67976200 & -5.12420600 & -2.21514700 \\
\hline $\mathrm{H}$ & -2.11696000 & 6.01035100 & -2.71193300 & $\mathrm{C}$ & 5.12985100 & -2.15486900 & -3.15905100 \\
\hline $\mathrm{C}$ & 3.67316700 & 4.26386000 & -0.07686100 & $\mathrm{H}$ & 5.77720800 & -2.52009600 & -2.33596500 \\
\hline $\mathrm{H}$ & 4.05849900 & 4.93426800 & -0.86035900 & $\mathrm{H}$ & 5.65457500 & -1.31787900 & -3.66381300 \\
\hline $\mathrm{C}$ & -1.37519900 & -1.01269800 & 3.83209500 & $\mathrm{H}$ & 5.03804000 & -2.98090800 & -3.89377200 \\
\hline
\end{tabular}




$\begin{array}{crrc}\text { C } & 3.45982100 & 1.80885200 & 4.27569100 \\ \text { H } & 4.46728200 & 1.56747700 & 3.88154100 \\ \text { H } & 3.24600800 & 1.11988700 & 5.11824800 \\ \text { H } & 3.50824900 & 2.83845300 & 4.68647700 \\ \text { C } & -4.63505500 & 1.57558600 & -3.69518300 \\ \text { H } & -5.40001500 & 1.73365600 & -2.90794900 \\ \text { H } & -4.90848400 & 0.65968800 & -4.25836200 \\ \text { H } & -4.70226700 & 2.43364000 & -4.39537400 \\ \text { C } & -2.18358700 & 1.16823200 & -4.20804100 \\ \text { H } & -2.16427800 & 1.96820300 & -4.97615900 \\ \text { H } & -2.42853200 & 0.22181500 & -4.73131100 \\ \text { H } & -1.15983500 & 1.07097800 & -3.79403300 \\ \text { C } & 2.88336200 & -1.18067700 & -3.81422400 \\ \text { H } & 2.67817100 & -1.97393900 & -4.56215800 \\ \text { H } & 3.40789700 & -0.35832100 & -4.34245000 \\ \text { H } & 1.91111700 & -0.79063000 & -3.45650400 \\ \text { C } & 3.34652300 & -4.59250400 & 2.44299600 \\ \text { H } & 3.29761000 & -5.66798600 & 2.17379400 \\ \text { H } & 3.30335300 & -4.52092700 & 3.54912500 \\ \text { H } & 4.33784300 & -4.21893300 & 2.11509700 \\ \text { C } & 4.02731600 & 2.77683500 & -2.77223400 \\ \text { H } & 2.96661900 & 2.48370000 & -2.89725600 \\ \text { H } & 4.60303300 & 2.32183700 & -3.60390300 \\ \text { H } & 4.09324600 & 3.87743100 & -2.89585700 \\ \text { C } & 6.09552600 & 2.66261300 & -1.31261400 \\ \text { H } & 6.25887900 & 3.75820000 & -1.37740800 \\ \text { H } & 6.66626100 & 2.19028100 & -2.13812600 \\ \text { H } & 6.53581100 & 2.32264000 & -0.35329900\end{array}$

trans-3a ${ }^{\text {++ }}$ with PBE

$\begin{array}{lrrc}\mathrm{P} & -0.01119500 & -1.33384400 & 0.08349800 \\ \mathrm{P} & -0.01074200 & 1.33400900 & -0.08343100 \\ \mathrm{~N} & 3.41466700 & -1.09497900 & -0.25859300 \\ \mathrm{~N} & -3.47081700 & 1.09555000 & -0.21211000 \\ \mathrm{~N} & 3.41508900 & 1.09392200 & 0.25825000 \\ \mathrm{~N} & -3.47110900 & -1.09435600 & 0.21295000 \\ \mathrm{C} & -2.65106200 & 0.00047300 & 0.00032900 \\ \mathrm{C} & 2.60579700 & -0.00038000 & -0.00012300 \\ \mathrm{C} & 3.05664100 & 2.28491500 & 0.98205600 \\ \mathrm{C} & 3.15489300 & -3.54927700 & -0.33042500 \\ \mathrm{C} & 3.05567800 & -2.28583800 & -0.98234400 \\ \mathrm{C} & 2.70430600 & 2.19009700 & 2.36184200 \\ \mathrm{C} & -3.09181100 & -2.43390200 & 0.55549400 \\ \mathrm{C} & 1.20885000 & -0.00015600 & 0.00002700\end{array}$

\begin{tabular}{|c|c|c|c|}
\hline C & 4.82428500 & -0.76510000 & 0.02366300 \\
\hline $\mathrm{H}$ & 5.48613100 & -1.22084100 & -0.73681800 \\
\hline F & 5.12871700 & -1.14779900 & 1.02297400 \\
\hline $\mathrm{C}$ & 3.15617400 & 3.54830600 & 0.33008100 \\
\hline C & 2.70303300 & -2.19085500 & -2.36204900 \\
\hline C & -3.25081000 & -2.85544800 & 1.90792300 \\
\hline $\mathrm{C}$ & -1.24987200 & 0.00028100 & 0.00023000 \\
\hline C & 4.82457800 & 0.76349600 & -0.02401800 \\
\hline $\mathrm{H}$ & 5.48659600 & 1.21897800 & 0.73646900 \\
\hline $\mathrm{H}$ & 5.12914700 & 1.14609500 & -1.02332500 \\
\hline $\mathrm{C}$ & -3.09118500 & 2.43488400 & -0.55509600 \\
\hline C & 3.62651200 & -3.69519800 & 1.11578200 \\
\hline $\mathrm{H}$ & 3.51451300 & -2.70054200 & 1.60059700 \\
\hline $\mathrm{C}$ & -3.25047800 & 2.85618600 & -1.90756400 \\
\hline $\mathrm{C}$ & 2.72453800 & 0.86785100 & 3.12790700 \\
\hline $\mathrm{H}$ & 2.59515800 & 0.04635000 & 2.39578900 \\
\hline $\mathrm{C}$ & -2.66095800 & 3.33437400 & 0.46342800 \\
\hline $\mathrm{C}$ & -2.66239400 & -3.33335600 & -0.46340300 \\
\hline $\mathrm{C}$ & 2.41089300 & 3.38138800 & 3.05125100 \\
\hline $\mathrm{H}$ & 2.13490100 & 3.33357600 & 4.11476300 \\
\hline 2 & -2.66624500 & 2.94766200 & 1.94127400 \\
\hline $\mathrm{H}$ & -2.66100900 & 1.83869400 & 1.99570000 \\
\hline $\mathrm{C}$ & 2.86173100 & -4.70770300 & -1.07729800 \\
\hline $\mathrm{H}$ & 2.93480300 & -5.69292000 & -0.59348700 \\
\hline $\mathrm{C}$ & -3.80460600 & -1.93384200 & 2.99316400 \\
\hline $\mathrm{H}$ & -3.87996100 & -0.91688400 & 2.55488000 \\
\hline $\mathrm{C}$ & -4.87294700 & 0.76017800 & 0.07408700 \\
\hline 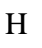 & -5.55193300 & 1.26729300 & -0.63721200 \\
\hline $\mathrm{H}$ & -5.15142900 & 1.08139500 & 1.10413100 \\
\hline $\mathrm{C}$ & -2.32280600 & 4.64764400 & 0.07995800 \\
\hline $\mathrm{H}$ & -1.97632000 & 5.35962100 & 0.84352100 \\
\hline $\mathrm{C}$ & 2.72375900 & -0.86859400 & -3.12808300 \\
\hline $\mathrm{H}$ & 2.59409100 & -0.04709800 & -2.39600400 \\
\hline S & -2.92096000 & -4.18709900 & 2.22732800 \\
\hline $\mathrm{H}$ & -3.03658800 & -4.53940800 & 3.26384300 \\
\hline $\mathrm{C}$ & 2.40890000 & -3.38200900 & -3.05138200 \\
\hline 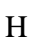 & 2.13260100 & -3.33407400 & -4.11480500 \\
\hline$c$ & -2.92016600 & 4.18762400 & -2.22739500 \\
\hline 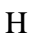 & -3.03603000 & 4.53974300 & -3.26394900 \\
\hline$C$ & 2.48602800 & 4.62887400 & 2.42042100 \\
\hline $\mathrm{H}$ & 2.26075100 & 5.54637100 & 2.98505000 \\
\hline $\mathrm{C}$ & 2.86367300 & 4.70685900 & 1.07700700 \\
\hline $\mathrm{H}$ & 2.93698800 & 5.69203800 & 0.59315500 \\
\hline $\mathrm{C}$ & -2.44766100 & -5.07038200 & 1.24868800 \\
\hline
\end{tabular}




\begin{tabular}{|c|c|c|c|c|c|c|c|}
\hline $\mathrm{H}$ & -2.18911500 & -6.10507800 & 1.52129600 & $\mathrm{C}$ & -1.43598900 & 3.45042800 & 2.71292700 \\
\hline $\mathrm{C}$ & 3.62750400 & 3.69399000 & -1.11624100 & $\mathrm{H}$ & -0.49277700 & 3.13650700 & 2.22371800 \\
\hline $\mathrm{H}$ & 3.51511900 & 2.69932800 & -1.60095600 & $\mathrm{H}$ & -1.44321700 & 3.04681100 & 3.74594600 \\
\hline $\mathrm{C}$ & -3.80492300 & 1.93456800 & -2.99246300 & $\mathrm{H}$ & -1.42578800 & 4.55644200 & 2.80197300 \\
\hline $\mathrm{H}$ & -3.88078100 & 0.91777100 & -2.55389200 & $\mathrm{C}$ & 5.12057300 & 4.08612700 & -1.16948500 \\
\hline $\mathrm{C}$ & -2.44606400 & 5.07090500 & -1.24914600 & $\mathrm{H}$ & 5.27313800 & 5.08988300 & -0.72206800 \\
\hline $\mathrm{H}$ & -2.18711600 & 6.10541100 & -1.52209300 & $\mathrm{H}$ & 5.47987300 & 4.12751800 & -2.21816300 \\
\hline $\mathrm{C}$ & 0.13663200 & -2.02311900 & 1.80502400 & $\mathrm{H}$ & 5.76699600 & 3.37994600 & -0.61327500 \\
\hline $\mathrm{H}$ & -0.57229100 & -2.85902500 & 1.95123600 & $\mathrm{C}$ & 2.80129800 & 4.70874800 & -1.92874500 \\
\hline $\mathrm{H}$ & -0.03561100 & -1.23231800 & 2.55934800 & $\mathrm{H}$ & 1.71193900 & 4.52361900 & -1.85441300 \\
\hline $\mathrm{H}$ & 1.16864800 & -2.40858600 & 1.90901200 & $\mathrm{H}$ & 3.08634700 & 4.66497800 & -2.99938700 \\
\hline $\mathrm{C}$ & 2.48370000 & -4.62953900 & -2.42058900 & $\mathrm{H}$ & 2.98580900 & 5.74891800 & -1.59145200 \\
\hline $\mathrm{H}$ & 2.25786200 & -5.54693100 & -2.98516400 & $\mathrm{C}$ & -5.22129600 & 2.37719900 & -3.41462600 \\
\hline $\mathrm{C}$ & -2.32474600 & -4.64688400 & -0.08037200 & $\mathrm{H}$ & -5.91405600 & 2.44619200 & -2.55135300 \\
\hline $\mathrm{H}$ & -1.97893400 & -5.35886000 & -0.84424500 & $\mathrm{H}$ & -5.65407600 & 1.66327700 & -4.14483200 \\
\hline $\mathrm{C}$ & 1.57738000 & 0.73539600 & 4.14350500 & $\mathrm{H}$ & -5.20090900 & 3.37644700 & -3.89653800 \\
\hline $\mathrm{H}$ & 1.69279200 & 1.43201400 & 4.99896200 & $\mathrm{C}$ & -5.22118600 & -2.37586000 & 3.41531400 \\
\hline $\mathrm{H}$ & 1.55712300 & -0.28894300 & 4.56760900 & $\mathrm{H}$ & -5.91403900 & -2.44428100 & 2.55207200 \\
\hline $\mathrm{H}$ & 0.59298100 & 0.93713800 & 3.67507000 & $\mathrm{H}$ & -5.65355800 & -1.66191800 & 4.14574300 \\
\hline $\mathrm{C}$ & 0.13690700 & 2.02264000 & -1.80524700 & $\mathrm{H}$ & -5.20125200 & -3.37525200 & 3.89694600 \\
\hline $\mathrm{H}$ & 1.16881000 & 2.40834300 & -1.90947800 & $\mathrm{C}$ & 4.08999900 & -0.66959400 & -3.81734200 \\
\hline $\mathrm{H}$ & -0.57222300 & 2.85832400 & -1.95173500 & $\mathrm{H}$ & 4.92998100 & -0.70668700 & -3.09436600 \\
\hline $\mathrm{H}$ & -0.03521200 & 1.23148800 & -2.55923000 & $\mathrm{H}$ & 4.13093800 & 0.30964800 & -4.33768000 \\
\hline $\mathrm{C}$ & -2.88457500 & -1.82847700 & 4.22280900 & $\mathrm{H}$ & 4.27196500 & -1.46196100 & -4.57239700 \\
\hline $\mathrm{H}$ & -2.77180300 & -2.80388200 & 4.73925100 & $\mathrm{C}$ & 1.57718900 & -0.73594300 & -4.14430800 \\
\hline $\mathrm{H}$ & -3.30957600 & -1.11707200 & 4.95973900 & $\mathrm{H}$ & 1.69305200 & -1.43244300 & -4.99980000 \\
\hline $\mathrm{H}$ & -1.87234100 & -1.47119800 & 3.95052800 & $\mathrm{H}$ & 1.55723800 & 0.28845400 & -4.56828100 \\
\hline $\mathrm{C}$ & -2.66802100 & -2.94632600 & -1.94116300 & $\mathrm{H}$ & 0.59250200 & -0.93768600 & -3.67648400 \\
\hline $\mathrm{H}$ & -2.66259100 & -1.83734900 & -1.99535100 & $\mathrm{C}$ & -2.88502800 & 1.82834100 & -4.22215000 \\
\hline $\mathrm{C}$ & -4.87317500 & -0.75859800 & -0.07311000 & $\mathrm{H}$ & -2.77176900 & 2.80355100 & -4.73885500 \\
\hline $\mathrm{H}$ & -5.55222400 & -1.26550300 & 0.63827700 & $\mathrm{H}$ & -3.31047100 & 1.11696500 & -4.95885300 \\
\hline $\mathrm{H}$ & -5.15186000 & -1.07975900 & -1.10312100 & $\mathrm{H}$ & -1.87297300 & 1.47059300 & -3.94982900 \\
\hline $\mathrm{C}$ & 4.09039700 & 0.66871400 & 3.81786800 & $\mathrm{C}$ & -3.96355400 & 3.44953600 & 2.61258400 \\
\hline $\mathrm{H}$ & 4.93073900 & 0.70563600 & 3.09529600 & $\mathrm{H}$ & -4.00586400 & 4.55834700 & 2.60715300 \\
\hline $\mathrm{H}$ & 4.13094200 & -0.31049800 & 4.33829300 & $\mathrm{H}$ & -4.01100600 & 3.11587800 & 3.66951700 \\
\hline $\mathrm{H}$ & 4.27211000 & 1.46110400 & 4.57296000 & $\mathrm{H}$ & -4.87420400 & 3.08502900 & 2.09643500 \\
\hline $\mathrm{C}$ & 5.11947600 & -4.08776300 & 1.16868200 & $\mathrm{C}$ & -1.43803700 & -3.44913900 & -2.71323000 \\
\hline $\mathrm{H}$ & 5.27167300 & -5.09151400 & 0.72112700 & $\mathrm{H}$ & -0.49466500 & -3.13535200 & -2.22424400 \\
\hline $\mathrm{H}$ & 5.47896900 & -4.12937300 & 2.21728600 & $\mathrm{H}$ & -1.44553400 & -3.04543700 & -3.74621500 \\
\hline $\mathrm{H}$ & 5.76599500 & -3.38170400 & 0.61242800 & $\mathrm{H}$ & -1.42794200 & -4.55514900 & -2.80234500 \\
\hline $\mathrm{C}$ & 2.80019500 & -4.70978100 & 1.92840200 & $\mathrm{C}$ & -3.96561000 & -3.44779500 & -2.61222000 \\
\hline $\mathrm{H}$ & 1.71087200 & -4.52436500 & 1.85428700 & $\mathrm{H}$ & -4.00812300 & -4.55660000 & -2.60708400 \\
\hline $\mathrm{H}$ & 3.08546400 & -4.66613100 & 2.99899000 & $\mathrm{H}$ & -4.01333200 & -3.11384400 & -3.66904800 \\
\hline $\mathrm{H}$ & 2.98437800 & -5.74998100 & 1.59102400 & $\mathrm{H}$ & -4.87602900 & -3.08326000 & -2.09567600 \\
\hline
\end{tabular}




\section{cis-3a with B3L YP+D3BJ}

P $\quad-0.14688800 \quad-1.37147300$

$\begin{array}{llll}\text { N } & 3.14473900 & -1.39270700 & -0.14429600\end{array}$

$\begin{array}{lrrr}\mathrm{N} & -3.21535900 & 1.36261900 & 0.72494500\end{array}$

$\begin{array}{lrrr}\mathrm{N} & 3.38827400 & 0.79100400 & 0.29369200\end{array}$

$\begin{array}{llll}\mathrm{N} & -3.29797800 & -0.85874400 & 1.03981200\end{array}$

$\begin{array}{lrrr}\text { C } & -2.50299900 & 0.16837500 & 0.52519900\end{array}$

$\begin{array}{lrrr}\text { C } & 2.45488300 & -0.17651400 & -0.07356700\end{array}$

$\begin{array}{llll}\text { C } & 3.13526500 & 2.14810800 & 0.64279400\end{array}$

$\begin{array}{llll}\text { C } & 2.49654200 & -3.68976600 & -0.65737700\end{array}$

C $\quad 2.86300200 \quad-2.40308200 \quad-1.10877400$

$\begin{array}{lrrr}\text { C } & 2.52958200 & 2.44887700 & 1.88553700\end{array}$

$\begin{array}{lrrr}\text { C } & -2.93202600 & -2.23300500 & 1.17852600\end{array}$

$\begin{array}{llll}\text { C } & 1.12429400 & -0.03600600 & -0.31865100\end{array}$

$\begin{array}{llll}\text { C } & 4.47042600 & -1.26580700 & 0.45733100\end{array}$

H $\quad 5.20547200 \quad-1.86946100 \quad-0.09236000$

$\begin{array}{llll}\mathrm{H} & 4.46573300 & -1.60195000 & 1.51079500\end{array}$

$\begin{array}{llll}\text { C } & 3.56654500 & 3.17437100 & -0.22810700\end{array}$

$\begin{array}{llll}\text { C } & 2.99582200 & -2.12261000 & -2.49012300\end{array}$

C -2.21403900

C -1.27449100

C 4.74028600

H $\quad 5.28068800$

H $\quad 5.32739600$

C -3.12185600

C 2.38287900

H 2.31516200

C -3.58686900

C 2.04983200

H 1.80102600

C -2.61964700

C -3.38683800

C 2.41823000

H $\quad 1.96737100$

C -2.19589300

H $\quad-2.04142200$

C 2.26304700

H $\quad 1.97498100$

C -1.58898800

H $\quad-1.72841100$

C $\quad-4.51266500$

H $\quad-5.32276200$

2.30883900

$-2.67769400$

0.07669700

0.23714900

0.63610300

0.47782000

2.46737400

$-3.99023400$

$-3.01761500$

2.33440500

1.34426700

0.47465000

3.69881500

$-3.14244500$

3.79220800

4.04806900

3.86623200

2.85277500

$-4.69112800$

$-5.69041800$

$-0.69318800$

1.09613900

1.11218700
H $\quad-4.75311800$

C -2.55746600

$-1.71407100$
$-0.05194800$

0.35570300

1.22657300

$-0.54845900$

$-0.18127700$

0.82743900

1.33667100

$-1.51093500$

2.81760500

2.19577400

0.29423900

0.19399100

2.25723000

3.21600600

1.74290700

2.14081500

$-1.60911600$

$-1.27743100$

3.30813900

2.92453600

1.34460800

0.59170200
H $\quad-2.16325100$

C 3.44654800

H 3.36420500

C $\quad-2.04126500$

H $\quad-1.49950700$

C 2.74556800

H $\quad 2.84023700$

C $\quad-3.50153400$

H $\quad-3.84453600$

C 2.86268600

H $\quad 2.76045800$

C $\quad 3.42047500$

H $\quad 3.75247400$

C -2.51680800

H $\quad-2.36143800$

C 4.21111100

H $\quad 4.03516100$

C -4.18920500

H $\quad-4.18868600$

1.84346300

4.78276100

5.73862500

$-0.75942300$

$-0.05588600$

$-4.05658500$

$-4.42228700$

$-3.15198400$

$-2.95376100$

3.44418200

3.35490000

4.81492300

5.85745900

4.50658200

5.31219400

$-4.96617200$

$-6.03742700$

2.86522300

1.80055400

1.04182400

0.30753200

4.65829300

5.51216100

$-1.84476700$

C -0.45810600

H -0.37006100

$-0.99350200$

$-2.24879700$

$-2.63142800$

Н $\quad 0.24634200$

C 2.38272700

$-4.42716100$

$-5.21805700$

$-4.50906400$

$-5.22999900$

1.72022000

2.55361300

0.86377700

2.00242300

1.99146500

2.46401900

2.74621900

1.17206400

$-1.94429800$

$-2.94996500$

$-1.21666000$

$-1.83892600$

$\begin{array}{llll}\text { C } & -4.10348700 & -2.63776800 & -1.05158200\end{array}$

2.11495500

$-0.59105700$

$-0.24202900$

$-2.99199600$

$-2.15852800$

2.48419200

3.35868900

$-3.40498400$

$-4.47465300$

$-2.36111300$

$-3.39432700$

1.41980200

1.73073200

0.18293500

$-0.47542700$

1.54462000

1.69377000

$-1.57179700$

$-1.77517700$

$-2.04201500$

$-1.22923800$

$-1.91063100$

$-2.58977100$

$-2.04027600$

$-2.72784600$

$-2.10422000$

$-2.34138800$

$-2.97223200$

$-3.70166000$

0.39584400

$-0.34892800$

3.57167200

4.27495700

4.16188300

2.86423200

$-1.98569700$

$-2.19573300$

$-2.11115400$

$-2.69948100$

3.41714000

3.80267500

4.10831900

2.43288100 


\begin{tabular}{|c|c|c|c|c|c|c|c|}
\hline $\mathrm{H}$ & -3.62984500 & -1.68180400 & -1.31434300 & $\mathrm{C}$ & -3.34882700 & 0.44579400 & -3.17867700 \\
\hline $\mathrm{C}$ & -4.31805100 & -0.30058200 & 1.92416100 & $\mathrm{H}$ & -3.29565800 & 1.12720800 & -4.04279100 \\
\hline $\mathrm{H}$ & -3.96510100 & -0.24772300 & 2.97046600 & $\mathrm{H}$ & -3.78690000 & -0.50316800 & -3.52698500 \\
\hline $\mathrm{H}$ & -5.23368300 & -0.90855300 & 1.90815100 & $\mathrm{H}$ & -2.32720000 & 0.24270300 & -2.83522400 \\
\hline $\mathrm{C}$ & 3.16767600 & 0.92343100 & 3.78449100 & $\mathrm{C}$ & -3.31503900 & 4.53330500 & 2.56060400 \\
\hline $\mathrm{H}$ & 4.06948400 & 0.60215100 & 3.24118800 & $\mathrm{H}$ & -3.50524600 & 5.55824100 & 2.20143900 \\
\hline $\mathrm{H}$ & 2.83890800 & 0.08493200 & 4.41992200 & $\mathrm{H}$ & -3.03702100 & 4.59446300 & 3.62553700 \\
\hline $\mathrm{H}$ & 3.45546600 & 1.75915500 & 4.44338000 & $\mathrm{H}$ & -4.26261200 & 3.97807700 & 2.48640400 \\
\hline $\mathrm{C}$ & 3.64255700 & -4.71108200 & 1.33577700 & $\mathrm{C}$ & -3.96188200 & -3.56886200 & -2.26067900 \\
\hline $\mathrm{H}$ & 3.75185900 & -5.69638800 & 0.85310000 & $\mathrm{H}$ & -2.91275400 & -3.84082600 & -2.44923700 \\
\hline $\mathrm{H}$ & 3.58777500 & -4.87285200 & 2.42478800 & $\mathrm{H}$ & -4.35130200 & -3.07234300 & -3.16326300 \\
\hline $\mathrm{H}$ & 4.55637800 & -4.13592300 & 1.12188900 & $\mathrm{H}$ & -4.53504000 & -4.50090100 & -2.13096000 \\
\hline $\mathrm{C}$ & 1.11488700 & -4.77513000 & 1.17287400 & $\mathrm{C}$ & -5.58990000 & -2.35606800 & -0.77999200 \\
\hline $\mathrm{H}$ & 0.22330400 & -4.24577600 & 0.81175200 & $\mathrm{H}$ & -6.11066400 & -3.27771100 & -0.47337300 \\
\hline $\mathrm{H}$ & 1.02260700 & -4.88734900 & 2.26444900 & $\mathrm{H}$ & -6.08346900 & -1.97183100 & -1.68699800 \\
\hline $\mathrm{H}$ & 1.12361000 & -5.78908100 & 0.74081300 & $\mathrm{H}$ & -5.72496700 & -1.61064100 & 0.01402700 \\
\hline $\mathrm{C}$ & -0.87905800 & 4.63269900 & 1.88609100 & & & & \\
\hline $\mathrm{H}$ & -0.08979700 & 4.15966800 & 1.28715600 & \multicolumn{4}{|c|}{ trans-3a with B3LYP+D3BJ } \\
\hline $\mathrm{H}$ & -0.55435400 & 4.63693200 & 2.93804700 & $\mathrm{P}$ & 0.03223100 & -1.38051000 & 0.13515300 \\
\hline $\mathrm{H}$ & -0.97710700 & 5.68472100 & 1.57256300 & $\mathrm{P}$ & 0.03871300 & 1.38209700 & 0.34679400 \\
\hline $\mathrm{C}$ & 5.73023600 & 3.10235500 & -1.52488600 & $\mathrm{~N}$ & 3.27601600 & -1.21560600 & -0.45887700 \\
\hline $\mathrm{H}$ & 5.95201800 & 4.17145900 & -1.37424900 & $\mathrm{~N}$ & -3.32644600 & 1.13771400 & 0.68590900 \\
\hline $\mathrm{H}$ & 6.20568800 & 2.78871400 & -2.46861000 & $\mathrm{~N}$ & 3.50949600 & 0.96460300 & 0.03978200 \\
\hline $\mathrm{H}$ & 6.20544900 & 2.54924800 & -0.70149900 & $\mathrm{~N}$ & -3.34415800 & -1.09249400 & 0.86885100 \\
\hline $\mathrm{C}$ & 3.58599400 & 3.65312300 & -2.73113300 & $\mathrm{C}$ & -2.51676400 & 0.00074500 & 0.59401200 \\
\hline $\mathrm{H}$ & 2.49775800 & 3.50679400 & -2.77716700 & $\mathrm{C}$ & 2.56724300 & -0.05489900 & -0.11601700 \\
\hline $\mathrm{H}$ & 4.01514200 & 3.32296600 & -3.69084000 & $\mathrm{C}$ & 3.23260200 & 2.26429600 & 0.54906800 \\
\hline $\mathrm{H}$ & 3.77621500 & 4.73433700 & -2.64153900 & $\mathrm{C}$ & 2.71888700 & -3.54643000 & -0.93253200 \\
\hline $\mathrm{C}$ & -5.65093300 & 1.24083100 & -2.47140200 & $\mathrm{C}$ & 2.78756200 & -2.20307800 & -1.36453500 \\
\hline $\mathrm{H}$ & -6.25978200 & 1.64805000 & -1.64884900 & $\mathrm{C}$ & 2.80937300 & 2.41574600 & 1.89167000 \\
\hline $\mathrm{H}$ & -6.09448800 & 0.28164500 & -2.78286600 & $\mathrm{C}$ & -2.92966500 & -2.44781000 & 0.99164700 \\
\hline $\mathrm{H}$ & -5.73251300 & 1.93729600 & -3.32107600 & $\mathrm{C}$ & 1.22313900 & 0.01286100 & 0.04295300 \\
\hline $\mathrm{C}$ & -2.25937900 & -1.80823500 & 4.68748900 & $\mathrm{C}$ & 4.70312100 & -1.02763300 & -0.21015200 \\
\hline $\mathrm{H}$ & -3.34607500 & -1.64227500 & 400 & $\mathrm{H}$ & 5.30153500 & 15500 & -0.974 \\
\hline $\mathrm{H}$ & -1.83669500 & -1.06013600 & 5.37759600 & $\mathrm{H}$ & 4.99647600 & -1.42299000 & 0.78027900 \\
\hline $\mathrm{H}$ & -2.10312000 & -2.80285800 & 5.13577800 & $\mathrm{C}$ & 3.38522100 & 3.38824600 & -0.29860400 \\
\hline $\mathrm{C}$ & 4.92288800 & -0.79422200 & -3.41746500 & $\mathrm{C}$ & 2.39502300 & -1.84436700 & -2.67847900 \\
\hline $\mathrm{H}$ & 5.56724000 & -1.15401400 & -2.59998900 & $\mathrm{C}$ & -2.90283300 & -3.03912900 & 2.27717000 \\
\hline $\mathrm{H}$ & 5.26909700 & 0.21158600 & -3.70743400 & $\mathrm{C}$ & -1.18521400 & -0.00260800 & 0.31251700 \\
\hline $\mathrm{H}$ & 5.07369600 & -1.46645400 & -4.27770200 & $\mathrm{C}$ & 4.86032300 & 0.49715100 & -0.26394000 \\
\hline $\mathrm{C}$ & 2.55308800 & -0.20477600 & -4.10702800 & $\mathrm{H}$ & 5.57809000 & 0.87605700 & 0.47889900 \\
\hline $\mathrm{H}$ & 2.62085100 & -0.80240600 & -5.03013700 & $\mathrm{H}$ & 5.19925100 & 0.82486200 & -1.26178700 \\
\hline $\mathrm{H}$ & 2.85281700 & 0.82549100 & -4.35693100 & $\mathrm{C}$ & -3.12020900 & 2.33958700 & -0.04791200 \\
\hline $\mathrm{H}$ & 1.50181800 & -0.18246300 & -3.78832200 & $\mathrm{C}$ & 3.10252100 & -3.94123900 & 0.48363800 \\
\hline
\end{tabular}




\begin{tabular}{|c|c|c|c|c|c|c|c|}
\hline $\mathrm{H}$ & 3.12300700 & -3.01233000 & 1.07267600 & $\mathrm{H}$ & 1.54790000 & -4.95818700 & -3.81769700 \\
\hline $\mathrm{C}$ & -3.39560400 & 2.36433000 & -1.43460400 & $\mathrm{C}$ & -2.11698400 & -4.50128500 & 0.00782700 \\
\hline $\mathrm{C}$ & 2.71866100 & 1.22063600 & 2.83029400 & $\mathrm{H}$ & -1.79626600 & -5.07469500 & -0.86220300 \\
\hline $\mathrm{H}$ & 2.49383300 & 0.33903000 & 2.21946000 & $\mathrm{C}$ & 1.58479600 & 1.33520900 & 3.85469500 \\
\hline 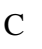 & -2.69042600 & 3.49837500 & 0.63643700 & $\mathrm{H}$ & 1.77427700 & 2.12066500 & 4.60438900 \\
\hline 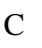 & -2.55458100 & -3.18097300 & -0.16104200 & $\mathrm{H}$ & 1.47510300 & 0.38528500 & 4.40108100 \\
\hline $\mathrm{C}$ & 2.54007300 & 3.70667900 & 2.36126100 & $\mathrm{H}$ & 0.63189400 & 1.55694700 & 3.35141800 \\
\hline $\mathrm{H}$ & 2.20446800 & 3.84362300 & 3.38969700 & $\mathrm{C}$ & 0.04024100 & 2.18656500 & -1.33429900 \\
\hline $\mathrm{C}$ & -2.42570200 & 3.46471100 & 2.13326900 & $\mathrm{H}$ & 1.03815200 & 2.62928300 & -1.44659400 \\
\hline 11 & -2.28414500 & 2.40694900 & 2.40009900 & $\mathrm{H}$ & -0.70262600 & 2.99049800 & -1.39469500 \\
\hline $\mathrm{C}$ & 2.28399200 & -4.52606000 & -1.83534100 & $\mathrm{H}$ & -0.12043800 & 1.46154100 & -2.14144800 \\
\hline $\mathrm{H}$ & 2.23168300 & -5.56782600 & -1.51310600 & $\mathrm{C}$ & -2.21960000 & -2.26723500 & 4.60696900 \\
\hline C & -3.30924000 & -2.26641700 & 3.52472600 & $\mathrm{H}$ & -2.01160800 & -3.28459900 & 4.97525300 \\
\hline $\mathrm{H}$ & -3.45792100 & -1.21924000 & 3.22577100 & $\mathrm{H}$ & -2.54099500 & -1.66315600 & 5.47071200 \\
\hline $\mathrm{C}$ & -4.63546400 & 0.80756800 & 1.24031000 & $\mathrm{H}$ & -1.27903100 & -1.84699900 & 4.22620200 \\
\hline $\mathrm{H}$ & -5.42590600 & 1.38733400 & 0.74255300 & $\mathrm{C}$ & -2.70472900 & -2.58333500 & -1.55371100 \\
\hline $\mathrm{H}$ & -4.68167400 & 1.02698600 & 2.32402200 & $\mathrm{H}$ & -2.52271400 & -1.50494200 & -1.46483700 \\
\hline $\mathrm{C}$ & -2.54592200 & 4.68620200 & -0.09197200 & $\mathrm{C}$ & -4.74451500 & -0.69573700 & 0.97590300 \\
\hline $\mathrm{H}$ & -2.20991600 & 5.59160500 & 0.41654700 & $\mathrm{H}$ & -5.25061900 & -1.23355100 & 1.78870500 \\
\hline $\mathrm{C}$ & 2.50393200 & -0.41491000 & -3.19207800 & $\mathrm{H}$ & -5.29826500 & -0.90483200 & 0.04139600 \\
\hline $\mathrm{H}$ & 2.44942100 & 0.25631300 & -2.32867200 & $\mathrm{C}$ & 4.07388300 & 0.98108600 & 3.51555900 \\
\hline $\mathrm{C}$ & -2.48972800 & -4.37238400 & 2.39074400 & $\mathrm{H}$ & 4.87656200 & 0.83443700 & 2.77624500 \\
\hline H & -2.46016900 & -4.84511900 & 3.37496500 & $\mathrm{H}$ & 4.03458700 & 0.08528900 & 4.15687200 \\
\hline $\mathrm{C}$ & 1.94587600 & -2.85423300 & -3.53646600 & $\mathrm{H}$ & 4.35552600 & 1.84028700 & 4.14632600 \\
\hline $\mathrm{H}$ & 1.63580500 & -2.59714300 & -4.55037600 & $\mathrm{C}$ & 4.51016700 & -4.55828300 & 0.52968200 \\
\hline $\mathrm{C}$ & -3.23643900 & 3.57386500 & -2.12249200 & $\mathrm{H}$ & 4.54593500 & -5.49029300 & -0.05798000 \\
\hline $\mathrm{H}$ & -3.43925700 & 3.61345700 & -3.19528000 & $\mathrm{H}$ & 4.79989900 & -4.80040800 & 1.56536100 \\
\hline $\mathrm{C}$ & 2.69046800 & 4.82100300 & 1.53839400 & $\mathrm{H}$ & 5.26717800 & -3.87665300 & 0.11384100 \\
\hline $\mathrm{H}$ & 2.47139400 & 5.81917500 & 1.92523300 & $\mathrm{C}$ & 2.07269000 & -4.88288100 & 1.12540800 \\
\hline $\mathrm{C}$ & 3.11450600 & 4.66028500 & 0.22144000 & $\mathrm{H}$ & 1.04932300 & -4.49966000 & 1.00780200 \\
\hline $\mathrm{H}$ & 3.22663200 & 5.53757400 & -0.41856500 & $\mathrm{H}$ & 2.27647800 & -4.99754300 & 2.20207100 \\
\hline $\mathrm{C}$ & -2.08548900 & -5.09406700 & 1.26870600 & $\mathrm{H}$ & 2.11022000 & -5.88909800 & 0.67774800 \\
\hline $\mathrm{H}$ & -1.74261800 & -6.12597700 & 1.37729400 & $\mathrm{C}$ & -1.15041000 & 4.21521500 & 2.53273500 \\
\hline $\mathrm{C}$ & 3.86491200 & 3.24057300 & -1.73656400 & $\mathrm{H}$ & -0.28683700 & 3.83988200 & 1.96605100 \\
\hline $\mathrm{H}$ & 3.71006500 & 2.18956900 & -2.01964200 & $\mathrm{H}$ & -0.94504200 & 4.06653100 & 3.60514800 \\
\hline $\mathrm{C}$ & -3.86368300 & 1.12578800 & -2.18399000 & $\mathrm{H}$ & -1.23988400 & 5.30116800 & 2.36620500 \\
\hline $\mathrm{H}$ & -3.92065800 & 0.30234300 & -1.46335000 & $\mathrm{C}$ & 5.36851900 & 3.55452200 & -1.84093100 \\
\hline $\mathrm{C}$ & -2.81791700 & 4.72645800 & -1.45913200 & $\mathrm{H}$ & 5.55398500 & 4.61724900 & -1.61507900 \\
\hline $\mathrm{H}$ & -2.69631900 & 5.66100200 & -2.01242200 & $\mathrm{H}$ & 5.74293900 & 3.35379700 & -2.85802600 \\
\hline C & 0.34921600 & -1.88594800 & 1.91685200 & $\mathrm{H}$ & 5.96373700 & 2.96093900 & -1.13251900 \\
\hline $\mathrm{H}$ & -0.29155800 & -2.72928500 & 2.20235100 & $\mathrm{C}$ & 3.08460800 & 4.09999600 & -2.74137100 \\
\hline $\mathrm{H}$ & 0.18001700 & -1.03845300 & 2.59630700 & $\mathrm{H}$ & 2.00129700 & 3.92811300 & -2.67355800 \\
\hline $\mathrm{H}$ & 1.39942400 & -2.19986800 & 1.99741500 & $\mathrm{H}$ & 3.40383800 & 3.86033600 & -3.76836400 \\
\hline $\mathrm{C}$ & 1.89744700 & -4.18656500 & -3.12750100 & $\mathrm{H}$ & 3.26637900 & 5.17550700 & -2.58814300 \\
\hline
\end{tabular}




\begin{tabular}{rrrr}
$\mathrm{C}$ & -5.27236200 & 1.31713000 & -2.76502700 \\
$\mathrm{H}$ & -5.99280700 & 1.60036100 & -1.98133700 \\
$\mathrm{H}$ & -5.62584600 & 0.38484100 & -3.23429000 \\
$\mathrm{H}$ & -5.29023800 & 2.10668700 & -3.53350100 \\
$\mathrm{C}$ & -4.63569900 & -2.79924500 & 4.09226500 \\
$\mathrm{H}$ & -5.43320200 & -2.80253700 & 3.33300700 \\
$\mathrm{H}$ & -4.97342300 & -2.18557700 & 4.94312600 \\
$\mathrm{H}$ & -4.52164100 & -3.83539100 & 4.45010800 \\
$\mathrm{C}$ & 3.86000100 & -0.18881200 & -3.87999000 \\
$\mathrm{H}$ & 4.69772100 & -0.43101300 & -3.20855100 \\
$\mathrm{H}$ & 3.96960400 & 0.86197800 & -4.19526100 \\
$\mathrm{H}$ & 3.95859100 & -0.82549800 & -4.77425200 \\
$\mathrm{C}$ & 1.34525300 & -0.01648700 & -4.11341900 \\
$\mathrm{H}$ & 1.39063600 & -0.52961700 & -5.08733600 \\
$\mathrm{H}$ & 1.37508700 & 1.06610100 & -4.31230200 \\
$\mathrm{H}$ & 0.37505300 & -0.25106000 & -3.65123400 \\
$\mathrm{C}$ & -2.86099100 & 0.69612800 & -3.26363300 \\
$\mathrm{H}$ & -2.71530000 & 1.48331800 & -4.02056200 \\
$\mathrm{H}$ & -3.21723800 & -0.20746400 & -3.78246600 \\
$\mathrm{H}$ & -1.88468400 & 0.46784900 & -2.81547500 \\
$\mathrm{C}$ & -3.64706300 & 3.99120400 & 2.90605700 \\
$\mathrm{H}$ & -3.82973300 & 5.05327900 & 2.67283500 \\
$\mathrm{H}$ & -3.48839900 & 3.90623200 & 3.99355600 \\
$\mathrm{H}$ & -4.56150200 & 3.43478700 & 2.64937500 \\
$\mathrm{C}$ & -1.70006000 & -3.12461800 & -2.57473800 \\
$\mathrm{H}$ & -0.67267500 & -3.04562000 & -2.19580200 \\
$\mathrm{H}$ & -1.76645900 & -2.54223300 & -3.50740000 \\
$\mathrm{H}$ & -1.90126300 & -4.17648000 & -2.83687000 \\
$\mathrm{C}$ & -4.14520600 & -2.78005700 & -2.05853400 \\
$\mathrm{H}$ & -4.37259200 & -3.85195400 & -2.17816700 \\
$\mathrm{H}$ & -4.28346400 & -2.29273900 & -3.03720300 \\
$\mathrm{H}$ & -4.88579700 & -2.36034000 & -1.36309900 \\
& & & \\
\hline
\end{tabular}

\section{cis-3a ${ }^{\cdot+}$ with B3LYP+D3BJ}

$\begin{array}{lrrc}\mathrm{P} & -0.13116200 & -1.25031800 & -0.19532200 \\ \mathrm{P} & 0.03831900 & 1.40403200 & 0.18551100 \\ \mathrm{~N} & 3.24196200 & -1.34541300 & -0.22957100 \\ \mathrm{~N} & -3.28777300 & 1.30989800 & 0.67152500 \\ \mathrm{~N} & 3.43485900 & 0.80760700 & 0.28299200 \\ \mathrm{~N} & -3.35583300 & -0.90189800 & 0.90553900 \\ \mathrm{C} & -2.57570900 & 0.14844600 & 0.49150200 \\ \mathrm{C} & 2.54386100 & -0.17187200 & -0.06532000 \\ \mathrm{C} & 3.13846400 & 2.17570600 & 0.57752400 \\ \mathrm{C} & 2.50810900 & -3.66433900 & -0.48261100\end{array}$

\begin{tabular}{|c|c|c|c|}
\hline & 2.85098200 & -2.42854300 & -107 \\
\hline $\mathrm{C}$ & 2.64984900 & 2.50791500 & 1.86197900 \\
\hline & -2.96016000 & -2.27133900 & 1.00866600 \\
\hline & 1.16 & 0 & -0.20007800 \\
\hline & 4.62493500 & -1.20704600 & 0.24098500 \\
\hline 1 & 5.31572300 & -1.70929000 & -0.44943900 \\
\hline & 4.74265900 & 00 & \\
\hline & 3.40358500 & 3.16178 & -0.40092300 \\
\hline C & 2.90142500 & -2.25893500 & -2.47492900 \\
\hline$C$ & -2.28777500 & -2.71850600 & 20900 \\
\hline 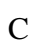 & -1.27197400 & 0.09583200 & 78500 \\
\hline & 3900 & 0.31 & 000 \\
\hline $\mathrm{H}$ & 5.35054600 & 0.65443600 & 1.17211800 \\
\hline त & 5.36227400 & 0.67761700 & -0.60696300 \\
\hline 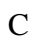 & 500 & 00 & -0 \\
\hline$C$ & 2.52912500 & -3.85138600 & 1.02499900 \\
\hline H & 2.54566500 & -2.84597500 & 1.47386900 \\
\hline $\mathrm{z}$ & -3.41439900 & 2.46519800 & -1.489 \\
\hline & 2.42445400 & 000 & 2.92659800 \\
\hline 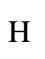 & 2.24942500 & 0.48738400 & 2.41032500 \\
\hline C & -2.64618800 & 3.66892500 & 0.52657600 \\
\hline $\mathrm{C}$ & -3.34609300 & -3.15778700 & -0.02691200 \\
\hline & 2.43192200 & 3.86023400 & 8400 \\
\hline 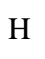 & 2.06274700 & 4.14919900 & 3.13228400 \\
\hline C & -2.36847600 & 3.70546700 & 2.02063300 \\
\hline H & -2.27720900 & 2.66090500 & 5000 \\
\hline $\mathrm{C}$ & 2.18506200 & -4.73205200 & -1.33113700 \\
\hline H & 1.91755000 & -5.69915000 & -0.90231900 \\
\hline c & -1.83117300 & -1.76442100 & 3.26256900 \\
\hline 1 & -2.02481500 & 00 & 9500 \\
\hline C & -4.63693300 & 1.02325700 & 1.17503400 \\
\hline $\mathrm{H}$ & -5.37112900 & 1.09518400 & 0.35325500 \\
\hline H & -4.92624700 & 1.73689000 & 1.95748300 \\
\hline & -2.50595900 & 4.82620000 & -0.251 \\
\hline $\mathrm{H}$ & -2.16138900 & 5.74966700 & 0.21641600 \\
\hline C & 3.33787900 & -0.95078100 & -3.11803500 \\
\hline Hi & 3.69079100 & -0.28795200 & -2.31767200 \\
\hline C & -2.05010000 & -4.09304500 & 296 \\
\hline $\mathrm{H}$ & -1.54425600 & -4.46957500 & 3.187 \\
\hline $\mathrm{C}$ & 2.55711800 & -3.35084800 & -3.28064700 \\
\hline $\mathrm{H}$ & 2.57939200 & -3.24586700 & -4.36717900 \\
\hline C & -3.25347400 & 64654700 & -2.22408 \\
\hline $\mathrm{H}$ & -3.48774700 & 3.65285400 & -3.29045400 \\
\hline & 2.67292100 & 4.84623900 & .1927250 \\
\hline
\end{tabular}




\begin{tabular}{|c|c|c|c|c|c|c|c|}
\hline $\mathrm{H}$ & 2.48927800 & 5.89511600 & 1.43620600 & $\mathrm{C}$ & 1.27394000 & -4.56088600 & 1.53957200 \\
\hline$C$ & 3.15216500 & 4.49949900 & -0.06839400 & I & 0.36421200 & -4.03849600 & 1.21587500 \\
\hline $\mathrm{H}$ & 3.34625500 & 5.28221300 & -0.80387300 & 1 & 1.27561400 & -4.59103000 & 2.63978900 \\
\hline $\mathrm{C}$ & -2.43842400 & -4.98400200 & 1.29987600 & I & 1.21426700 & -5.60190000 & 1.18533800 \\
\hline$H$ & -2.24241500 & -6.05200400 & 1.41841400 & $\mathrm{C}$ & -1.05121900 & 4.41341300 & 2.35376900 \\
\hline $\mathrm{C}$ & 4.02332000 & 2.81733400 & -1.75003800 & $\mathrm{H}$ & -0.21682000 & 3.97119500 & 1.79389400 \\
\hline $\mathrm{H}$ & 3.92458500 & 1.73143200 & -1.88971800 & $\mathrm{H}$ & -0.83072200 & 4.31982000 & 3.42835800 \\
\hline $\mathrm{C}$ & -3.93057400 & 1.21276300 & -2.18634800 & $\mathrm{H}$ & -1.09342900 & 5.48947600 & 2.12272600 \\
\hline $\mathrm{H}$ & -4.07895800 & 0.43428400 & -1.42773200 & 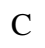 & 5.52229200 & 3.17235400 & -1.74724600 \\
\hline $\mathrm{C}$ & -2.80235400 & 4.81629700 & -1.61335300 & $\mathrm{H}$ & 5.65948600 & 4.26359200 & -1.68719900 \\
\hline $\mathrm{H}$ & -2.68478400 & 5.72806000 & -2.20330200 & $\mathrm{H}$ & 6.00901500 & 2.82182700 & -2.67118000 \\
\hline $\mathrm{C}$ & -0.43932600 & -2.14457900 & -1.77746600 & H & 6.05120100 & 2.73168900 & -0.88941900 \\
\hline $\mathrm{H}$ & -0.30957300 & -1.46211100 & -2.62664700 & $\mathrm{C}$ & 3.33288200 & 3.48429900 & -2.94776800 \\
\hline $\mathrm{H}$ & -1.47320300 & -2.49895300 & -1.73789900 & $\mathrm{H}$ & 2.27025500 & 3.21486500 & -3.01536000 \\
\hline $\mathrm{H}$ & 0.23121700 & -3.00451500 & -1.87097100 & 11 & 3.81978300 & 3.16669800 & -3.88300000 \\
\hline$C$ & 2.20225900 & -4.57639100 & -2.71675000 & I & 3.40366900 & 4.58193900 & -2.90339500 \\
\hline $\mathrm{H}$ & 1.94462800 & -5.41872400 & -3.36277200 & $\mathrm{C}$ & -5.29628500 & 1.45085500 & -2.84771000 \\
\hline $\mathrm{C}$ & -3.06507700 & -4.51855400 & 0.14306600 & $\mathrm{H}$ & -6.03018100 & 1.84085500 & -2.12546100 \\
\hline $\mathrm{H}$ & -3.34751500 & -5.23075200 & -0.63230600 & $\mathrm{H}$ & -5.69001200 & 0.50921100 & -3.26192600 \\
\hline $\mathrm{C}$ & 1.19127800 & 1.70775400 & 3.79755700 & $\mathrm{H}$ & -5.22809400 & 2.17334300 & -3.67568500 \\
\hline $\mathrm{H}$ & 1.30556100 & 2.61383400 & 4.41224600 & $C$ & -2.62120500 & -1.99183600 & 4.56192700 \\
\hline $\mathrm{H}$ & 1.03588800 & 0.86769500 & 4.49094300 & $\mathrm{H}$ & -3.70732800 & -1.91791600 & 4.39878300 \\
\hline $\mathrm{H}$ & 0.29042300 & 1.82029300 & 5800 & $\mathrm{H}$ & 1800 & -1.24970 & 2400 \\
\hline $\mathrm{C}$ & 0.05697800 & 2.27004100 & -1.46149400 & $H$ & -2.41972700 & -2.99196200 & 4.97692700 \\
\hline $\mathrm{H}$ & 0.99934100 & 2.82893600 & -1.49987000 & $\mathrm{C}$ & 4.51765100 & -1.15323400 & -4.08003700 \\
\hline $\mathrm{H}$ & -0.77197900 & 2.98490800 & -1.50174100 & U & 5.35703000 & -1.66504700 & -3.58438400 \\
\hline $\mathrm{H}$ & -0.00204600 & 1.57737800 & -2.31032900 & 1 & 4.87881300 & -0.18097700 & -4.45073400 \\
\hline $\mathrm{C}$ & -0.31980600 & -1.86247000 & 3.51927500 & I & 4.23126900 & -1.75391700 & -4.95721600 \\
\hline $\mathrm{H}$ & -0.03625200 & -2.85672700 & 3.89610100 & $\mathrm{C}$ & 2.16773100 & -0.23374500 & -3.80674900 \\
\hline $\mathrm{H}$ & -0.01739700 & -1.12241100 & 4.27482300 & 1 & 1.74699600 & -0.84539800 & -4.62066100 \\
\hline $\mathrm{H}$ & 0.25409700 & -1.67036100 & 2.60103500 & 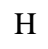 & 2.50112900 & 0.72169000 & -4.24064900 \\
\hline $\mathrm{C}$ & -4.05393700 & -2.64858100 & -1.28028400 & $\mathrm{H}$ & 1.36756600 & -0.01489300 & -3.08649200 \\
\hline $\mathrm{H}$ & -3.61967800 & -1.66359500 & -1.51088500 & $\mathrm{C}$ & -2.90912800 & 0.66020900 & -3.19171700 \\
\hline $\mathrm{C}$ & -4.49182400 & -0.40304600 & 1.6934 & $\mathrm{H}$ & -2.70025400 & 000 & -3.99239500 \\
\hline $\mathrm{H}$ & -4.24705300 & -0.41901500 & 2.76880200 & $\mathrm{H}$ & -3.29108500 & -0.25893900 & -3.66386900 \\
\hline $\mathrm{H}$ & -5.38431300 & -1.02124300 & 1.53918600 & $\mathrm{H}$ & -1.96218800 & 0.41995700 & -2.68955400 \\
\hline$C$ & 3.68385500 & 1.28032800 & 3.79526800 & $r$ & -3.54570000 & 4.34817300 & 2.77422800 \\
\hline $\mathrm{H}$ & 4.56994500 & 1.03807700 & 3.18949000 & $\mathrm{H}$ & -3.66874000 & 5.40307900 & 2.48106600 \\
\hline П & 3.54709600 & 0.47554500 & 4.53493900 & $\mathrm{H}$ & -3.37460600 & 4.31804500 & 3.86195000 \\
\hline $\mathrm{H}$ & 3.90318500 & 2.21103900 & 4.34220400 & $\mathrm{H}$ & -4.49784500 & 3.83743500 & 2.56352400 \\
\hline $\mathrm{C}$ & 3.80486100 & -4.59172800 & 1.46119900 & $\mathrm{C}$ & -3.85208000 & -3.54511000 & -2.50882800 \\
\hline $\mathrm{H}$ & 3.82304700 & -5.61350500 & 1.04946300 & $\mathrm{H}$ & -2.79381200 & -3.78545700 & -2.68877300 \\
\hline $\mathrm{H}$ & 3.85456600 & -4.66909500 & 2.55882000 & $\mathrm{H}$ & -4.24083500 & -3.03999600 & -3.40607200 \\
\hline $\mathrm{H}$ & 4.71470400 & -4.08052400 & 1.11211900 & $\mathrm{H}$ & -4.39863500 & -4.49597700 & -2.41228100 \\
\hline
\end{tabular}




$$
\begin{array}{llll}
\mathrm{C} & -5.56061200 & -2.43987600 & -1.05025000 \\
\mathrm{H} & -6.04647700 & -3.38817500 & -0.77190500 \\
\mathrm{H} & -6.03983200 & -2.06983800 & -1.97016200 \\
\mathrm{H} & -5.76439500 & -1.71119500 & -0.25634500
\end{array}
$$

\section{trans-3a ${ }^{\cdot+}$ with B3LYP+D3BJ}

$\begin{array}{llll}\text { P } & 0.02034500 & -1.30446100 & 0.21701300\end{array}$

$\begin{array}{llll}\mathrm{P} & 0.01300600 & 1.36289700 & 0.15226300\end{array}$

$\begin{array}{llll}\text { N } & 3.25068400 & -1.20021200 & -0.49328500\end{array}$

$\begin{array}{lrrr}\mathrm{N} & -3.39358200 & 1.11461900 & 0.53861600\end{array}$

$\begin{array}{lrrr}\mathrm{N} & 3.48706900 & 0.95747500 & 0.03016800\end{array}$

$\begin{array}{llll}\mathrm{N} & -3.33305500 & -1.07524600 & 0.88299900\end{array}$

$\begin{array}{lrrr}\text { C } & -2.57075200 & 0.01917100 & 0.55850700\end{array}$

$\begin{array}{llll}\text { C } & 2.57976700 & -0.04882500 & -0.15172000\end{array}$

$\begin{array}{llll}\text { C } & 3.18362700 & 2.26368900 & 0.52750700\end{array}$

$\begin{array}{llll}\text { C } & 2.68103600 & -3.56154600 & -0.83447500\end{array}$

$\begin{array}{llll}\text { C } & 2.71383700 & -2.23860200 & -1.32455400\end{array}$

$\begin{array}{lrrr}\text { C } & 2.79433800 & 2.41285700 & 1.87855200\end{array}$

$\begin{array}{llll}\text { C } & -2.87340700 & -2.42094500 & 1.00894100\end{array}$

$\begin{array}{llll}\text { C } & 1.20746700 & 0.03220400 & 0.00247600\end{array}$

$\begin{array}{llll}\text { C } & 4.69804200 & -1.02595300 & -0.30450700\end{array}$

$\mathrm{H} \quad 5.24848000 \quad-1.47052800 \quad-1.14385300$

$\begin{array}{llll}\mathrm{H} & 5.03111600 & -1.51829400 & 0.62390000\end{array}$

$\begin{array}{llll}\text { C } & 3.29928500 & 3.37533000 & -0.34093300\end{array}$

$\begin{array}{llll}\text { C } & 2.27424300 & -1.92420000 & -2.63586400\end{array}$

$\begin{array}{lrrr}\text { C } & -2.80243500 & -2.99124500 & 2.30003500\end{array}$

$\begin{array}{llll}\text { C } & -1.20674100 & 0.02794500 & 0.30023400\end{array}$

$\begin{array}{llll}\text { C } & 4.85835800 & 0.49771900 & -0.22875600\end{array}$

$\begin{array}{llll}\mathrm{H} & 5.52497600 & 0.81509400 & 0.58512700\end{array}$

$\begin{array}{lrrr}\mathrm{H} & 5.24335300 & 0.91617500 & -1.17067100\end{array}$

$\begin{array}{lrrr}\text { C } & -3.10840000 & 2.36853700 & -0.08000600\end{array}$

$\begin{array}{llll}\text { C } & 3.18258400 & -3.91972400 & 0.55546300\end{array}$

$\begin{array}{llll}\mathrm{H} & 3.22537500 & -2.98456200 & 1.13572700\end{array}$

$\begin{array}{lrrr}\text { C } & -3.40375900 & 2.52079200 & -1.45411800\end{array}$

$\begin{array}{llll}\text { C } & 2.74877200 & 1.23575900 & 2.84321900\end{array}$

$\begin{array}{llll}\mathrm{H} & 2.72376700 & 0.31180100 & 2.25220000\end{array}$

$\begin{array}{lrrr}\text { C } & -2.60368500 & 3.43054300 & 0.70142400\end{array}$

$\begin{array}{llll}\text { C } & -2.53556100 & -3.15585800 & -0.15308200\end{array}$

$\begin{array}{lrrr}\text { C } & 2.50494000 & 3.70286400 & 2.33926500\end{array}$

$\begin{array}{llll}\mathrm{H} & 2.20518300 & 3.84668700 & 3.37815000\end{array}$

$\begin{array}{lrrr}\text { C } & -2.37620600 & 3.26954800 & 2.19662200\end{array}$

$\begin{array}{llll}\mathrm{H} & -2.27658600 & 2.19122700 & 2.39481300\end{array}$

$\begin{array}{llll}\text { C } & 2.20153900 & -4.57255300 & -1.67969800\end{array}$

$\mathrm{H} \quad 2.17227000 \quad-5.60244400 \quad-1.32079900$
C $\quad-3.22981700$

H -3.39425600

$-2.22395200$

$-1.17819200$

C -4.73438200

0.78086200

H $\quad-5.50212100$

1.28274100

H $\quad-4.85506300$

1.10305900

C $\quad-2.37662700$

4.65963700

5.49844500

$-0.51307700$

C 2.35712500

0.19617600

H 2.27016600

$-4.31264800$

C -2.35019300

H $\quad-2.28815500$

$-4.77960600$

C 1.79769200

$-2.96895900$

$-2.75650500$

H 1.45089100

C -3.16827600

3.77091700

3.91957200

4.80757000

C 2.59719300

H 2.36412900

5.80468300

4.64352900

5.51751500

H $\quad 3.07715300$

$-5.03382300$

C -1.96059200

H $\quad-1.59232600$

$-6.05650800$

3.23159200

2.17862600

H $\quad 3.67924900$

1.37036700

C -3.94401800

H -4.26428700

0.57679000

4.82932300

5.79669900

H $\quad-2.47938000$

C $\quad 0.39110600$

$-1.92531500$

$-2.80658500$

H $\quad-0.21277100$

$-1.12779500$

$-2.20010200$

$-4.28241100$

$-5.08112100$

$-4.46102300$

$-5.04307000$

1.23178800

2.07757600

0.30962000

1.28767000

2.21656200

2.64854400

3.02798100

1.51297400

$-2.21238000$
3.54346300

3.24384900

1.03010800

0.42582600

2.07885000

0.06736300

0.64317400

$-3.20652500$

$-2.37556600$

2.41115200

3.39593800

$-3.43441400$

$-4.44590800$

$-2.03983900$

$-3.09962700$

1.49530600

1.87542000

0.17038400

$-0.47763800$

1.28312000

1.39157000

$-1.76883700$

$-2.06158200$

$-2.29295700$

$-1.60394900$

$-1.28792000$

$-1.76298100$

1.92816000

2.16729000

2.65968300

1.95042000

$-2.96693400$

$-3.61258400$

0.01512200

$-0.86045300$

3.71836700

4.42290200

4.31846600

3.10173600

$-1.48323000$

$-1.57971000$

$-1.50752600$

$-2.30448700$

4.64174300 


\begin{tabular}{|c|c|c|c|c|c|c|c|}
\hline $\mathrm{H}$ & -1.94112800 & -3.22646700 & 5.01281900 & $\mathrm{C}$ & 1.20742300 & -0.17937100 & -4.16486900 \\
\hline $\mathrm{H}$ & -2.50109700 & -1.61618100 & 5.50104000 & $\mathrm{H}$ & 1.28006200 & -0.73405700 & -5.11293000 \\
\hline $\mathrm{H}$ & -1.21442600 & -1.77673500 & 4.28217400 & $\mathrm{H}$ & 1.22622400 & 0.89218100 & -4.41641400 \\
\hline $\mathrm{C}$ & -2.78038700 & -2.59498100 & -1.54725000 & $\mathrm{H}$ & 0.23068800 & -0.40970300 & -3.71308400 \\
\hline $\mathrm{H}$ & -2.80415000 & -1.50093000 & -1.46625300 & $\mathrm{C}$ & -2.84753500 & 0.76370600 & -3.18337800 \\
\hline $\mathrm{C}$ & -4.76179900 & -0.74165600 & 0.89447100 & $\mathrm{H}$ & -2.44349800 & 1.51299500 & -3.88244800 \\
\hline $\mathrm{H}$ & -5.27188400 & -1.23644200 & 1.73016900 & $\mathrm{H}$ & -3.25217300 & -0.07048100 & -3.77752800 \\
\hline $\mathrm{H}$ & -5.24247500 & -1.06980200 & -0.04340500 & $\mathrm{H}$ & -2.01633400 & 0.37666600 & -2.57814500 \\
\hline $\mathrm{C}$ & 4.02684800 & 1.20156400 & 3.69796400 & $\mathrm{C}$ & -3.59824400 & 3.78396700 & 2.97811600 \\
\hline $\mathrm{H}$ & 4.92917300 & 1.15054600 & 3.06869300 & $\mathrm{H}$ & -3.73029900 & 4.86676400 & 2.82361100 \\
\hline $\mathrm{H}$ & 4.02618900 & 0.32656800 & 4.36734900 & $\mathrm{H}$ & -3.47174000 & 3.60893000 & 4.05823600 \\
\hline $\mathrm{H}$ & 4.10980700 & 2.10588900 & 4.32139300 & $\mathrm{H}$ & -4.52806300 & 3.29088300 & 2.65724900 \\
\hline $\mathrm{C}$ & 4.60686900 & -4.49968700 & 0.49183100 & $\mathrm{C}$ & -1.68360900 & -2.94611000 & -2.55668400 \\
\hline $\mathrm{H}$ & 4.61272900 & -5.44630900 & -0.07135500 & $\mathrm{H}$ & -0.69112600 & -2.66009500 & -2.18540000 \\
\hline $\mathrm{H}$ & 4.99077100 & -4.70604600 & 1.50348300 & $\mathrm{H}$ & -1.86285900 & -2.41764700 & -3.50580300 \\
\hline $\mathrm{H}$ & 5.30881500 & -3.81684300 & -0.00855100 & $\mathrm{H}$ & -1.66203900 & -4.02290100 & -2.78636500 \\
\hline $\mathrm{C}$ & 2.24493000 & -4.88914800 & 1.29138800 & $\mathrm{C}$ & -4.15768900 & -3.05358300 & -2.06013000 \\
\hline $\mathrm{H}$ & 1.19733600 & -4.55968200 & 1.24798500 & $\mathrm{H}$ & -4.18025600 & -4.14680500 & -2.19319400 \\
\hline $\mathrm{H}$ & 2.53768600 & -4.97420600 & 2.34944200 & $\mathrm{H}$ & -4.38201700 & -2.58900300 & -3.03361100 \\
\hline $\mathrm{H}$ & 2.29475900 & -5.90115300 & 0.86072300 & $\mathrm{H}$ & -4.96578300 & -2.79159300 & -1.36090900 \\
\hline $\mathrm{C}$ & -1.09159800 & 3.94790100 & 2.6840 & & & & \\
\hline $\mathrm{H}$ & -0.22303200 & 3.62230000 & 2.09574200 & \multicolumn{4}{|c|}{ cis-3a with M06-2X } \\
\hline $\mathrm{H}$ & -0.90556300 & 3.69283800 & 3.73873000 & $\mathrm{P}$ & -0.10827900 & -1.41178600 & -0.01634700 \\
\hline $\mathrm{H}$ & -1.15830700 & 5.04559000 & 2.62307200 & $\mathrm{P}$ & 0.05940900 & 1.34633700 & -0.32997100 \\
\hline $\mathrm{C}$ & 5.31484300 & 3.56739900 & -1.82642200 & $\mathrm{~N}$ & 3.41009500 & -1.16562700 & -0.45272900 \\
\hline $\mathrm{H}$ & 5.47452800 & 4.63544300 & -1.60986400 & $\mathrm{~N}$ & -3.17845600 & 1.45105000 & 0.51831700 \\
\hline $\mathrm{H}$ & 5.72427000 & 3.36031100 & -2.82778900 & $\mathrm{~N}$ & 3.32139600 & 1.06909700 & -0.22073900 \\
\hline $\mathrm{H}$ & 5.89862700 & 2.99721100 & -1.09000200 & $\mathrm{~N}$ & -3.39097700 & -0.74416100 & 0.87595900 \\
\hline $\mathrm{C}$ & 3.05900100 & 4.08914200 & -2.79621200 & $\mathrm{C}$ & -2.50269600 & 0.22658100 & 0.41414100 \\
\hline $\mathrm{H}$ & 1.97377200 & 3.91753100 & -2.77273200 & $\mathrm{C}$ & 2.53291000 & -0.08919500 & -0.30765300 \\
\hline $\mathrm{H}$ & 3.41609500 & 3.85516600 & -3.81110100 & $\mathrm{C}$ & 3.11737900 & 2.11147800 & 0.73390300 \\
\hline $\mathrm{H}$ & 3.23188900 & 5.16413000 & -2.63471100 & $\mathrm{C}$ & 2.57894000 & -3.43617700 & -0.07856300 \\
\hline $\mathrm{C}$ & -5.17276100 & 1.77552700 & -3.11833500 & $\mathrm{C}$ & 3.02655100 & -2.43604000 & -0.96821500 \\
\hline $\mathrm{H}$ & -5.95359300 & 2.22405200 & -2.48496800 & $\mathrm{C}$ & 3.11737000 & 5000 & 46400 \\
\hline $\mathrm{H}$ & -5.60197600 & 0.89456500 & -3.62042600 & $\mathrm{C}$ & -3.10852300 & -2.13561700 & 1.00252600 \\
\hline $\mathrm{H}$ & -4.91755700 & 2.50542100 & -3.90224800 & $\mathrm{C}$ & 1.17666100 & -0.11419100 & -0.29043300 \\
\hline $\mathrm{C}$ & -4.55672400 & -2.78024100 & 4.08914700 & $\mathrm{C}$ & 4.80214700 & -0.73267800 & -0.47059700 \\
\hline $\mathrm{H}$ & -5.34407500 & -2.79317000 & 3.31965300 & $\mathrm{H}$ & 5.35002000 & -1.22257600 & -1.28938200 \\
\hline $\mathrm{H}$ & -4.91405100 & -2.17527400 & 4.93721600 & $\mathrm{H}$ & 5.31744600 & -0.97851400 & 0.47492400 \\
\hline $\mathrm{H}$ & -4.43176400 & -3.81549800 & 4.44413600 & $\mathrm{C}$ & 3.01752000 & 3.43785300 & 0.26791000 \\
\hline $\mathrm{C}$ & 3.72052400 & -0.27567800 & -3.87699500 & $\mathrm{C}$ & 3.10124000 & -2.66684100 & -2.35557900 \\
\hline $\mathrm{H}$ & 4.55312400 & -0.45293000 & -3.17985800 & $\mathrm{C}$ & -2.59236500 & -2.65004500 & 2.20523500 \\
\hline $\mathrm{H}$ & 3.79995200 & 0.76016700 & -4.24419600 & $\mathrm{C}$ & -1.23234200 & 0.05616300 & -0.04092300 \\
\hline $\mathrm{H}$ & 3.85915600 & -0.95264800 & -4.73457700 & $\mathrm{C}$ & 4.68336400 & 0.78327200 & -0.66081200 \\
\hline
\end{tabular}




\begin{tabular}{|c|c|c|c|c|c|c|c|}
\hline $\mathrm{H}$ & 5.40594300 & 1.34283800 & -0.05181600 & $\mathrm{H}$ & -0.57299600 & -1.39261700 & -2.47031100 \\
\hline $\mathrm{H}$ & 4.83007500 & 1.07038500 & -1.71827200 & $\mathrm{H}$ & -1.26809100 & -2.81370100 & -1.62511000 \\
\hline $\mathrm{C}$ & -2.93049200 & 2.53753000 & -0.37744100 & $\mathrm{H}$ & 0.47189800 & -2.77381700 & -1.98025900 \\
\hline $\mathrm{C}$ & 2.53725100 & -3.17305200 & 1.41924400 & $\mathrm{C}$ & 2.30430600 & -4.92564500 & -1.97753800 \\
\hline 14 & 2.18695400 & -2.13745300 & 1.55253000 & $\mathrm{H}$ & 2.01629400 & -5.90167000 & -2.37171100 \\
\hline $\mathrm{C}$ & -3.38708100 & 2.46255900 & -1.70896300 & $\mathrm{C}$ & -3.16448600 & -4.34921500 & 0.04803600 \\
\hline $\mathrm{C}$ & 3.27492400 & 0.42540000 & 2.67383200 & $\mathrm{H}$ & -3.36629600 & -5.02318100 & -0.78438700 \\
\hline $\mathrm{H}$ & 3.49217600 & -0.26024300 & 1.84451500 & $\mathrm{C}$ & 1.97535600 & -0.05385400 & 3.32413900 \\
\hline $\mathrm{C}$ & -2.26681400 & 3.68337700 & 0.10647800 & $\mathrm{H}$ & 1.70110100 & 0.58706000 & 4.17766200 \\
\hline$c$ & -3.39869500 & -2.97971500 & -0.09399100 & $\mathrm{H}$ & 2.08605400 & -1.08454800 & 3.69755600 \\
\hline $\mathrm{C}$ & 2.99183200 & 2.89827300 & 3.01112200 & $\mathrm{H}$ & 1.14710700 & -0.03620000 & 2.59929200 \\
\hline $\mathrm{H}$ & 2.97740500 & 2.69448400 & 4.08420200 & $\mathrm{C}$ & 0.01753000 & 1.64722400 & -2.17449200 \\
\hline $\mathrm{C}$ & -1.84935600 & 3.78307000 & 1.56270900 & $\mathrm{H}$ & 1.00827400 & 2.01978900 & -2.47502300 \\
\hline $\mathrm{H}$ & -1.75514000 & 2.75274500 & 1.93532800 & $\mathrm{H}$ & -0.73379700 & 2.41444000 & -2.41418600 \\
\hline $\mathrm{C}$ & 2.20960700 & -4.67452000 & -0.61062400 & $\mathrm{H}$ & -0.19525600 & 0.72618200 & -2.73230200 \\
\hline$H$ & 1.84065300 & -5.45737500 & 0.05159200 & $\mathrm{C}$ & -0.80427500 & -2.01410600 & 3.86002300 \\
\hline $\mathrm{C}$ & -2.22957700 & -1.74999700 & 3.37328900 & $\mathrm{H}$ & -0.71745000 & -3.00444900 & 4.33386900 \\
\hline $\mathrm{H}$ & -2.25466800 & -0.71028600 & 3.01470800 & $\mathrm{H}$ & -0.50846400 & -1.26033400 & 4.60523100 \\
\hline $\mathrm{C}$ & -4.53919200 & 1.25843300 & 1.00218900 & $\mathrm{H}$ & -0.10144100 & -1.97032900 & 3.01671500 \\
\hline $\mathrm{H}$ & -5.26951500 & 1.28142900 & 0.17200400 & $\mathrm{C}$ & -3.93975500 & -2.39893000 & -1.39338900 \\
\hline $\mathrm{H}$ & -4.82151300 & 2.03780700 & 900 & $\mathrm{H}$ & -3.33097700 & -1.50510300 & -1.61288900 \\
\hline $\mathrm{C}$ & -2.04942300 & 4.74597900 & -0.77500900 & $\mathrm{C}$ & -4.46167000 & -0.12315900 & 1.64041100 \\
\hline $\mathrm{H}$ & -1.52506500 & 5.63591600 & -0.42329500 & $\mathrm{H}$ & -4.21132300 & -0.04238000 & 2.71285500 \\
\hline $\mathrm{C}$ & 3.48750300 & -1.57425300 & -3.33889900 & $\mathrm{H}$ & -5.39484800 & -0.69715500 & 1.55095900 \\
\hline $\mathrm{H}$ & 3.78481300 & -0.68634100 & -2.76508100 & $\mathrm{C}$ & 4.45421700 & 0.33408600 & 3.64774400 \\
\hline $\mathrm{C}$ & -2.39324900 & -4.03042800 & 2.30971900 & $\mathrm{H}$ & 5.38575400 & 0.68580100 & 3.17950000 \\
\hline $\mathrm{H}$ & -1.99246400 & -4.44709900 & 3.23614700 & $\mathrm{H}$ & 4.60198000 & -0.70753000 & 3.97004800 \\
\hline $\mathrm{C}$ & 2.74815200 & -3.93070700 & -2.84041900 & $\mathrm{H}$ & 4.28243100 & 0.94012100 & 4.55012300 \\
\hline $\mathrm{H}$ & 2.79680800 & -4.12747500 & -3.91378400 & $\mathrm{C}$ & 3.94199900 & -3.27815900 & 2.02374900 \\
\hline $\mathrm{C}$ & -3.16206400 & 3.55640600 & -2.55164500 & $\mathrm{H}$ & 4.33714200 & -4.29967100 & 1.90906500 \\
\hline$\Pi$ & -3.50646100 & 3.51458700 & -3.58758500 & $\mathrm{H}$ & 3.91567600 & -3.04119200 & 3.09882100 \\
\hline $\mathrm{C}$ & 2.92086000 & 4.21284800 & 2.56386500 & $\mathrm{H}$ & 4.64470000 & -2.58771000 & 1.53833600 \\
\hline $\mathrm{H}$ & 2.84346800 & 5.03211500 & 3.28069500 & $\mathrm{C}$ & 1.56939800 & -4.08580800 & 2.16717600 \\
\hline$c$ & 2.94266400 & 4.47684000 & 1.19897400 & П & 0.55518500 & -4.01880500 & 1.74378200 \\
\hline $\mathrm{H}$ & 2.87534800 & 5.50829300 & 0.84588800 & $\mathrm{H}$ & 1.52027900 & -3.78412600 & 3.22393000 \\
\hline$C$ & -2.67319000 & -4.87330000 & 1.24201100 & $\mathrm{H}$ & 1.90203100 & -5.13543300 & 2.14571400 \\
\hline $\mathrm{H}$ & -2.50218900 & -5.94719000 & 1.33413500 & $\mathrm{C}$ & -0.49992500 & 4.46990900 & 1.75567000 \\
\hline $\mathrm{C}$ & 2.98744800 & 3.75417400 & -1.21593200 & $\mathrm{H}$ & 0.27795500 & 3.96545100 & 1.16611600 \\
\hline П & 2.87981300 & 2.79374800 & -1.74124400 & $\mathrm{H}$ & -0.20150700 & 4.42231000 & 2.81390600 \\
\hline $\mathrm{C}$ & -4.10504800 & 1.24088100 & -2.26169400 & $\mathrm{H}$ & -0.53219600 & 5.53481900 & 1.47363800 \\
\hline $\mathrm{H}$ & -4.21354400 & 0.50601700 & -1.45310500 & $\mathrm{C}$ & 4.29971300 & 4.39964900 & -1.67237200 \\
\hline$C$ & -2.49321000 & 4.68593100 & -2.09380700 & $\mathrm{H}$ & 4.45787700 & 5.36253700 & -1.16166900 \\
\hline $\mathrm{H}$ & -2.31520600 & 5.52541000 & -2.76815500 & $\mathrm{H}$ & 4.28556700 & 4.58951600 & -2.75614800 \\
\hline $\mathrm{C}$ & -0.39252800 & -2.15600700 & -1.70073500 & $\mathrm{H}$ & 5.16278500 & 3.75662700 & -1.44531600 \\
\hline
\end{tabular}




\begin{tabular}{rrrr}
$\mathrm{C}$ & 1.78389900 & 4.63012300 & -1.57771100 \\
$\mathrm{H}$ & 0.84791900 & 4.19299300 & -1.19756000 \\
$\mathrm{H}$ & 1.69769400 & 4.73503400 & -2.67017400 \\
$\mathrm{H}$ & 1.88473300 & 5.64160800 & -1.15323100 \\
$\mathrm{C}$ & -5.50843100 & 1.60509800 & -2.75699300 \\
$\mathrm{H}$ & -6.10333100 & 2.08166400 & -1.96348900 \\
$\mathrm{H}$ & -6.04400600 & 0.70712100 & -3.09927600 \\
$\mathrm{H}$ & -5.46039700 & 2.30715600 & -3.60305900 \\
$\mathrm{C}$ & -3.23375500 & -1.90738400 & 4.52122100 \\
$\mathrm{H}$ & -4.26643500 & -1.71702100 & 4.19442800 \\
$\mathrm{H}$ & -2.99594000 & -1.21710600 & 5.34449800 \\
$\mathrm{H}$ & -3.19763900 & -2.93348800 & 4.91898400 \\
$\mathrm{C}$ & 4.67029900 & -1.98696800 & -4.21907100 \\
$\mathrm{H}$ & 5.53949900 & -2.28567600 & -3.61425100 \\
$\mathrm{H}$ & 4.97215900 & -1.15470800 & -4.87186700 \\
$\mathrm{H}$ & 4.40645100 & -2.83696800 & -4.86646400 \\
$\mathrm{C}$ & 2.27795000 & -1.16376700 & -4.18484000 \\
$\mathrm{H}$ & 1.90655100 & -2.01226600 & -4.78041300 \\
$\mathrm{H}$ & 2.54834700 & -0.35206500 & -4.87682300 \\
$\mathrm{H}$ & 1.46073500 & -0.81302700 & -3.53969900 \\
$\mathrm{C}$ & -3.28864500 & 0.55848700 & -3.36188400 \\
$\mathrm{H}$ & -3.11060200 & 1.23645200 & -4.21103800 \\
$\mathrm{H}$ & -3.82379400 & -0.32713300 & -3.73962700 \\
$\mathrm{H}$ & -2.31555000 & 0.23283900 & -2.96846100 \\
$\mathrm{C}$ & -2.94427300 & 4.49161100 & 2.37049200 \\
$\mathrm{H}$ & -3.06390600 & 5.53075400 & 2.02579400 \\
$\mathrm{H}$ & -2.68507000 & 4.51413300 & 3.43966400 \\
$\mathrm{H}$ & -3.91771300 & 3.99225800 & 2.26158000 \\
$\mathrm{C}$ & -3.82603900 & -3.34898200 & -2.58574000 \\
$\mathrm{H}$ & -2.80722200 & -3.73834400 & -2.71918100 \\
$\mathrm{H}$ & -4.10844700 & -2.82102900 & -3.50808900 \\
$\mathrm{H}$ & -4.50840400 & -4.20624100 & -2.47827700 \\
$\mathrm{C}$ & -5.40145000 & -1.95667900 & -1.25210400 \\
$\mathrm{H}$ & -6.03873000 & -2.81832400 & -1.00000200 \\
$\mathrm{H}$ & -5.76264200 & -1.53284900 & -2.20208400 \\
$\mathrm{H}$ & -5.52886200 & -1.19386300 & -0.47410800 \\
\hline & & &
\end{tabular}

\section{trans-3a with M06-2X}

$\begin{array}{rrrr}\mathrm{P} & -0.08207700 & -1.30608100 & -0.31264000 \\ \mathrm{P} & 0.03862700 & 1.43669700 & 0.01210400 \\ \mathrm{~N} & 3.34354400 & -1.04491900 & -0.69724900 \\ \mathrm{~N} & -3.29445600 & 1.35125300 & 0.47490200 \\ \mathrm{~N} & 3.36084100 & 1.13305800 & -0.15837000 \\ \mathrm{~N} & -3.41425900 & -0.87252500 & 0.74475300\end{array}$

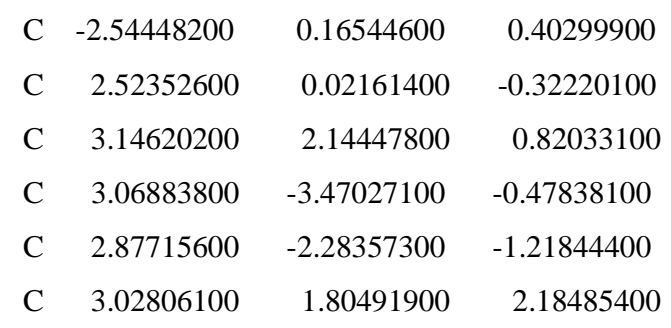

$\begin{array}{llll}\text { C } & -3.05274000 & -2.24216400 & 0.89417900\end{array}$

$\begin{array}{llll}\text { C } & 1.18208000 & 0.01434300 & -0.15892600\end{array}$

$\begin{array}{llll}\text { C } & 4.75134800 & -0.69126600 & -0.58190100\end{array}$

$\begin{array}{llll}\mathrm{H} & 5.33313000 & -1.14058200 & -1.39980900\end{array}$

$\begin{array}{llll}\mathrm{H} & 5.18692100 & -1.02616100 & 0.37834200\end{array}$

$\begin{array}{llll}\text { C } & 3.09278800 \quad 3.49187200 & 0.40673900\end{array}$

$\begin{array}{llll}\text { C } & 2.21624500 & -2.30532600 & -2.46672000\end{array}$

$\begin{array}{llll}\text { C } & -2.92140700 & -2.81779600 & 2.17663500\end{array}$

$\begin{array}{llll}\text { C } & -1.22852700 & 0.08675800 & 0.07281800\end{array}$

$\begin{array}{llll}\text { C } & 4.69975100 & 0.83605900 & -0.65406900\end{array}$

$\begin{array}{llll}\mathrm{H} & 5.45961200 & 1.31647800 & -0.02272900\end{array}$

$\mathrm{H} \quad 4.83159300 \quad 1.19110600 \quad-1.69291000$

$\begin{array}{llll}\text { C } & -3.00282200 & 2.47612500 & -0.36082400\end{array}$

$\begin{array}{llll}\text { C } & 3.80821300 & -3.46583200 & 0.85024100\end{array}$

$\mathrm{H} \quad 3.73915200 \quad-2.44249600 \quad 1.25541400$

$\begin{array}{llll}\text { C } & -3.31246400 & 2.42619100 & -1.73622300\end{array}$

$\begin{array}{llll}\text { C } & 3.13135200 & 0.37714800 & 2.69559500\end{array}$

$\begin{array}{llll}\mathrm{H} & 3.21839900 & -0.29825700 & 1.83533100\end{array}$

$\begin{array}{llll}\text { C } & -2.45299100 & 3.63486100 & 0.22501400\end{array}$

$\begin{array}{llll}\text { C } & -2.86297300 & -3.01793000 & -0.27267200\end{array}$

$\begin{array}{llll}\text { C } & 2.83267200 & 2.83163900 & 3.11238800\end{array}$

$\begin{array}{llll}\mathrm{H} & 2.73334800 & 2.58063800 & 4.17090200\end{array}$

$\begin{array}{llll}\text { C } & -2.13042600 & 3.67893600 & 1.70900700\end{array}$

$\begin{array}{llll}\mathrm{H} & -1.93368100 & 2.64291600 & 2.01861200\end{array}$

$\begin{array}{llll}\text { C } & 2.58942300 & -4.67306000 & -1.00575400\end{array}$

H $\quad 2.72687500 \quad-5.59890700 \quad-0.44495500$

$\begin{array}{llll}\text { C } & -3.04361500 & -2.00607900 & 3.45736400\end{array}$

$\mathrm{H} \quad-2.96496600 \quad-0.94363600 \quad 3.18300400$

$\begin{array}{llll}\text { C } & -4.70393400 & 1.06370100 & 0.71371200\end{array}$

$\begin{array}{llll}\mathrm{H} & -5.28673000 & 1.12176100 & -0.22272800\end{array}$

$\begin{array}{llll}\mathrm{H} & -5.14368700 & 1.77591500 & 1.42591700\end{array}$

$\begin{array}{llll}\text { C } & -2.24006800 & 4.75228900 & -0.58774100\end{array}$

$\begin{array}{llll}\mathrm{H} & -1.80793300 & 5.65567900 & -0.15519600\end{array}$

$\begin{array}{llll}\text { C } & 2.07173600 & -1.04310300 & -3.30061400\end{array}$

$\mathrm{H} \quad 1.97319000 \quad-0.19570500 \quad-2.61041700$

$\begin{array}{llll}\text { C } & -2.66104500 & -4.18909500 & 2.26831200\end{array}$

$\mathrm{H} \quad-2.57288000 \quad-4.65269200 \quad 3.25277200$

$\begin{array}{llll}\text { C } & 1.74282900 & -3.52835900 & -2.94729500\end{array}$ 


\begin{tabular}{|c|c|c|c|c|c|c|c|}
\hline $\mathrm{H}$ & 1.21561400 & -3.56281200 & -3.90129200 & $\mathrm{C}$ & 5.28913400 & -3.81310400 & 0.63895900 \\
\hline $\mathrm{C}$ & -3.10143000 & 3.57510400 & -2.50580200 & $\mathrm{H}$ & 5.38010200 & -4.84927900 & 0.27788100 \\
\hline $\mathrm{H}$ & -3.33797400 & 3.55704800 & -3.57221900 & $\mathrm{H}$ & 5.84941400 & -3.72970600 & 1.58262500 \\
\hline $\mathrm{C}$ & 2.78359500 & 4.16384400 & 2.71768900 & $\mathrm{H}$ & 5.76401000 & -3.16208900 & -0.10672500 \\
\hline $\mathrm{H}$ & 2.63768800 & 4.95050300 & 3.45983200 & $\mathrm{C}$ & 3.20742500 & -4.41885200 & 1.88800800 \\
\hline $\mathrm{C}$ & 2.92130100 & 4.48769100 & 1.37302600 & $\mathrm{H}$ & 2.12564300 & -4.26995800 & 2.00511900 \\
\hline $\mathrm{H}$ & 2.88250500 & 5.53421500 & 1.06416700 & $\mathrm{H}$ & 3.68753300 & -4.25827300 & 2.86475700 \\
\hline $\mathrm{C}$ & -2.47022700 & -4.96113300 & 1.12940800 & $\mathrm{H}$ & 3.37925600 & -5.47023000 & 1.61303600 \\
\hline $\mathrm{H}$ & -2.24588100 & -6.02511100 & 1.22093500 & $\mathrm{C}$ & -0.88326400 & 4.50250500 & 2.02658200 \\
\hline $\mathrm{C}$ & 3.24405100 & 3.87775000 & -1.05335100 & $\mathrm{H}$ & -0.02622900 & 4.16146200 & 1.42741300 \\
\hline $\mathrm{H}$ & 3.08756800 & 2.96180700 & -1.64336300 & $\mathrm{H}$ & -0.61819400 & 4.38281300 & 3.08772000 \\
\hline $\mathrm{C}$ & -3.83886600 & 1.17111700 & -2.41851000 & $\mathrm{H}$ & -1.04369700 & 5.57773500 & 1.84980800 \\
\hline $\mathrm{H}$ & -3.97011700 & 0.38889700 & -1.65860700 & $\mathrm{C}$ & 4.66277700 & 4.38400500 & -1.34063900 \\
\hline $\mathrm{C}$ & -2.57338800 & 4.72998100 & -1.93953100 & $\mathrm{H}$ & 4.87243200 & 5.29343900 & -0.75613700 \\
\hline $\mathrm{H}$ & -2.40655200 & 5.61426600 & -2.55700800 & $\mathrm{H}$ & 4.78155000 & 4.62902000 & -2.40695600 \\
\hline $\mathrm{C}$ & 0.20725400 & -2.24653900 & 1.27161700 & $\mathrm{H}$ & 5.42166500 & 3.63479900 & -1.07286300 \\
\hline $\mathrm{H}$ & -0.28966600 & -3.22644900 & 1.24648800 & $\mathrm{C}$ & 2.20329400 & 4.91297700 & -1.48982900 \\
\hline $\mathrm{H}$ & -0.14329300 & -1.66353600 & 2.13175800 & $\mathrm{H}$ & 1.18792600 & 4.61100300 & -1.19294600 \\
\hline $\mathrm{H}$ & 1.29429200 & -2.38891200 & 1.35581800 & $\mathrm{H}$ & 2.22185700 & 5.03622100 & -2.58302700 \\
\hline $\mathrm{C}$ & 1.92636300 & -4.70427900 & -2.22700800 & $\mathrm{H}$ & 2.40882000 & 5.89871900 & -1.04544200 \\
\hline $\mathrm{H}$ & 1.54762500 & -5.64978800 & -2.61914800 & $\mathrm{C}$ & -5.20016600 & 1.41962700 & -3.07650600 \\
\hline $\mathrm{C}$ & -2.54887900 & -4.37188800 & -0.12939500 & $\mathrm{H}$ & -5.92891700 & 1.82322800 & -2.35778400 \\
\hline $\mathrm{H}$ & -2.37624000 & -4.98094100 & -1.01653700 & $\mathrm{H}$ & -5.60364500 & 0.48306700 & -3.48989600 \\
\hline $\mathrm{C}$ & 1.86386900 & -0.02097200 & 3.45643600 & $\mathrm{H}$ & -5.11542800 & 2.14059800 & -3.90353400 \\
\hline $\mathrm{H}$ & 1.77110500 & 0.54668700 & 4.39548100 & $\mathrm{C}$ & -4.39753800 & -2.26159000 & 4.13510900 \\
\hline $\mathrm{H}$ & 1.88287700 & -1.09231000 & 3.71013600 & $\mathrm{H}$ & -5.24214200 & -2.10153900 & 3.45094200 \\
\hline $\mathrm{H}$ & 0.97293600 & 0.17855400 & 2.84264700 & $\mathrm{H}$ & -4.52711900 & -1.60802600 & 5.01088400 \\
\hline $\mathrm{C}$ & 0.09490600 & 2.00648000 & -1.76647200 & $\mathrm{H}$ & -4.44982300 & -3.30552700 & 4.48114700 \\
\hline $\mathrm{H}$ & 1.10077100 & 2.42073800 & -1.92906400 & $\mathrm{C}$ & 3.33889800 & -0.82352600 & -4.13460700 \\
\hline $\mathrm{H}$ & -0.64422600 & 2.79592100 & -1.95805700 & $\mathrm{H}$ & 4.23155800 & -0.77844400 & -3.49278900 \\
\hline $\mathrm{H}$ & -0.05165900 & 1.16800900 & -2.46104400 & $\mathrm{H}$ & 3.27479900 & 0.11731000 & -4.70201000 \\
\hline $\mathrm{C}$ & -1.91505900 & -2.29023300 & 4.45518000 & $\mathrm{H}$ & 3.48102200 & -1.64860600 & -4.84989200 \\
\hline $\mathrm{H}$ & -1.97895600 & -3.31249400 & 4.85652500 & $\mathrm{C}$ & 0.81998200 & -1.03944500 & -4.17757200 \\
\hline $\mathrm{H}$ & -1.98964300 & -1.59926600 & 5.30770600 & $\mathrm{H}$ & 0.88993900 & -1.76558200 & -5.00236700 \\
\hline $\mathrm{H}$ & -0.92460400 & -2.16563000 & 3.99805300 & $\mathrm{H}$ & 0.68106000 & -0.04580400 & -4.62950000 \\
\hline $\mathrm{C}$ & -3.06786400 & -2.40613100 & -1.64977400 & $\mathrm{H}$ & -0.07227900 & -1.27758800 & -3.57754400 \\
\hline $\mathrm{H}$ & -2.65428500 & -1.38831300 & -1.61991600 & $\mathrm{C}$ & -2.82938500 & 0.62595100 & -3.43371000 \\
\hline $\mathrm{C}$ & -4.67118400 & -0.35632600 & 1.26709800 & $\mathrm{H}$ & -2.59081400 & 1.37507300 & -4.20465800 \\
\hline $\mathrm{H}$ & -4.68775600 & -0.33427400 & 2.36808300 & $\mathrm{H}$ & -3.23963800 & -0.26198300 & -3.93948400 \\
\hline $\mathrm{H}$ & -5.51972200 & -0.96946100 & 0.92745400 & $\mathrm{H}$ & -1.89583500 & 0.33481900 & -2.93231100 \\
\hline $\mathrm{C}$ & 4.38655000 & 0.18540900 & 3.55250000 & $\mathrm{C}$ & -3.34190800 & 4.19078600 & 2.49664700 \\
\hline $\mathrm{H}$ & 5.29557600 & 0.44966800 & 2.99137000 & $\mathrm{H}$ & -3.58630600 & 5.22360400 & 2.20223700 \\
\hline $\mathrm{H}$ & 4.47346100 & -0.86295700 & 3.87802100 & $\mathrm{H}$ & -3.13478000 & 4.18292600 & 3.57727300 \\
\hline $\mathrm{H}$ & 4.35615400 & 0.81648900 & 4.45405900 & $\mathrm{H}$ & -4.23070700 & 3.57084600 & 2.31231500 \\
\hline
\end{tabular}




\begin{tabular}{|c|c|c|c|c|c|c|c|}
\hline $\mathrm{C}$ & -2.33664400 & -3.15423500 & -2.76247400 & $\mathrm{C}$ & 1.71894700 & -3.51115200 & 2.01498500 \\
\hline $\mathrm{H}$ & -1.27277100 & -3.28266100 & -2.51055500 & $\mathrm{H}$ & 1.67999500 & -2.41955900 & 2.15637900 \\
\hline $\mathrm{H}$ & -2.40113600 & -2.58083500 & -3.69956400 & $\mathrm{C}$ & -2.03593200 & 5.09769300 & -0.66334200 \\
\hline $\mathrm{H}$ & -2.78280000 & -4.14291600 & -2.95416200 & $\mathrm{H}$ & -1.85174700 & 5.98078200 & -0.04886100 \\
\hline $\mathrm{C}$ & -4.56744400 & -2.30102000 & -1.95390900 & $\mathrm{C}$ & 2.32107300 & 1.59012100 & 3.09000000 \\
\hline $\mathrm{H}$ & -5.02603800 & -3.30158500 & -1.98550600 & $\mathrm{H}$ & 2.24370800 & 0.54509300 & 2.74859000 \\
\hline $\mathrm{H}$ & -4.73330300 & -1.81852400 & -2.92994600 & $\mathrm{C}$ & 4.61259700 & -1.57056400 & 0.58878700 \\
\hline \multirow[t]{2}{*}{$\mathrm{H}$} & -5.09014100 & -1.71032600 & -1.18713100 & $\mathrm{H}$ & 5.16995100 & -1.62592500 & -0.36164600 \\
\hline & & & & $\mathrm{H}$ & 4.96342800 & -2.37816500 & 1.24198300 \\
\hline \multicolumn{4}{|c|}{ 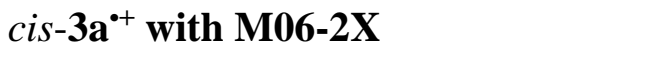 } & $\mathrm{C}$ & 1.43114600 & -4.91972100 & -0.08065900 \\
\hline $\mathrm{P}$ & 0.24256600 & 1.25884600 & 0.08871800 & $\mathrm{H}$ & 0.85748800 & -5.63761200 & 0.50565200 \\
\hline $\mathrm{P}$ & -0.19265100 & -1.36904000 & -0.05047800 & $\mathrm{C}$ & -2.75666000 & 1.62094100 & -3.15068200 \\
\hline $\mathrm{N}$ & -3.18658600 & 1.59506000 & -0.29368700 & $\mathrm{H}$ & -2.54417000 & 0.72212500 & -2.55166500 \\
\hline $\mathrm{N}$ & 3.17275100 & -1.67358000 & 0.34957900 & $\mathrm{C}$ & 2.57862500 & 3.83783200 & 1.94163100 \\
\hline $\mathrm{N}$ & -3.48076700 & -0.57242400 & 0.03432700 & $\mathrm{H}$ & 2.06670000 & 4.30114500 & 2.78664100 \\
\hline $\mathrm{N}$ & 3.54588700 & 0.47887600 & 0.67375300 & $\mathrm{C}$ & -2.07864900 & 4.04824800 & -2.83734000 \\
\hline $\mathrm{C}$ & 2.58921300 & -0.43440400 & 0.32277400 & $\mathrm{H}$ & -1.92942200 & 4.12174000 & -3.91503600 \\
\hline $\mathrm{C}$ & -2.53559600 & 0.40581500 & -0.11808700 & $\mathrm{C}$ & 2.39236400 & -4.26555200 & -2.19602500 \\
\hline $\mathrm{C}$ & -3.30558300 & -1.86306100 & 0.62321400 & $\mathrm{H}$ & 2.57682300 & -4.47721100 & -3.25127600 \\
\hline $\mathrm{C}$ & -2.41864900 & 3.89746600 & -0.05261400 & $\mathrm{C}$ & -3.16223100 & -4.37395800 & 1.80573700 \\
\hline $\mathrm{C}$ & -2.66448700 & 2.78504700 & -0.87617900 & $\mathrm{H}$ & -3.10565500 & -5.35913100 & 2.27127300 \\
\hline $\mathrm{C}$ & -3.03023800 & -1.95748500 & 2.00229700 & $\mathrm{C}$ & -3.43093300 & -4.26105000 & 0.44661300 \\
\hline $\mathrm{C}$ & 3.38642900 & 1.89149600 & 0.78630400 & $\mathrm{H}$ & -3.59290900 & -5.16314500 & -0.14523500 \\
\hline $\mathrm{C}$ & -1.16162600 & 0.21905100 & -0.10245000 & $\mathrm{C}$ & 3.10914100 & 4.64379600 & 0.93989700 \\
\hline $\mathrm{C}$ & -4.60142400 & 1.46608000 & 0.05750900 & $\mathrm{H}$ & 2.99114800 & 5.72697900 & 0.99614200 \\
\hline $\mathrm{H}$ & -5.22022700 & 2.10280500 & -0.58716300 & $\mathrm{C}$ & -3.91212300 & -2.91572800 & -1.63392800 \\
\hline $\mathrm{H}$ & -4.76845000 & 1.76027700 & 1.10889700 & $\mathrm{H}$ & -3.62664400 & -1.91153600 & -1.99041100 \\
\hline $\mathrm{C}$ & -3.51885600 & -3.00876500 & -0.16904900 & $\mathrm{C}$ & 3.64855100 & -2.10504500 & -2.51847300 \\
\hline $\mathrm{C}$ & -2.48081500 & 2.83471700 & -2.27594300 & $\mathrm{H}$ & 3.85035200 & -1.19233300 & -1.93720700 \\
\hline $\mathrm{C}$ & 2.73007200 & 2.44610300 & 1.90193200 & $\mathrm{C}$ & 1.67213400 & -5.17419500 & -1.42904100 \\
\hline $\mathrm{C}$ & 1.25489500 & -0.18684800 & 0.00697000 & $\mathrm{H}$ & 1.29462100 & -6.09145100 & -1.88368000 \\
\hline $\mathrm{C}$ & -4.82941300 & -0.02520100 & -0.15520700 & $\mathrm{C}$ & 0.60030600 & 2.56888400 & -1.14471100 \\
\hline $\mathrm{H}$ & -5.52091100 & -0.46632500 & 0.57423600 & $\mathrm{H}$ & 0.51224400 & 2.15028700 & -2.15669500 \\
\hline $\mathrm{H}$ & -5.19756700 & -0.24369600 & -1.17200100 & $\mathrm{H}$ & 1.62350600 & 2.91807900 & -0.96484000 \\
\hline $\mathrm{C}$ & 2.63326600 & -2.84627600 & -0.27010900 & $\mathrm{H}$ & -0.09208600 & 3.40721700 & -1.01401700 \\
\hline $\mathrm{C}$ & -2.54450000 & 3.81275700 & 1.45787800 & $\mathrm{C}$ & -1.87480500 & 5.17503000 & -2.04210200 \\
\hline $\mathrm{H}$ & -2.60787300 & 2.74336500 & 1.71992200 & $\mathrm{H}$ & -1.57744400 & 6.11787900 & -2.50355200 \\
\hline $\mathrm{C}$ & 2.87592400 & -3.07654000 & -1.63820000 & $\mathrm{C}$ & 3.81870100 & 4.07932100 & -0.11874100 \\
\hline $\mathrm{C}$ & -2.84165400 & -0.74034800 & 2.89205100 & $\mathrm{H}$ & 4.25552100 & 4.72989500 & -0.87652600 \\
\hline $\mathrm{H}$ & -2.90445600 & 0.16441000 & 2.27026100 & $\mathrm{C}$ & -1.45921100 & -0.73958600 & 3.54925200 \\
\hline $\mathrm{C}$ & 1.91949900 & -3.76191200 & 0.52948900 & $\mathrm{H}$ & -1.33125900 & -1.61621300 & 4.20250500 \\
\hline $\mathrm{C}$ & 3.96746800 & 2.69411500 & -0.21925600 & $\mathrm{H}$ & -1.33580100 & 0.15966400 & 4.16911900 \\
\hline $\mathrm{C}$ & -2.96566700 & -3.23042300 & 2.57263000 & $\mathrm{H}$ & -0.65758000 & -0.75798100 & 2.79305200 \\
\hline $\mathrm{H}$ & -2.75669800 & -3.32900500 & 3.64007700 & $\mathrm{C}$ & -0.33712500 & -1.71450100 & -1.87398000 \\
\hline
\end{tabular}




\begin{tabular}{|c|c|c|c|}
\hline $\mathrm{H}$ & -1.39072000 & -1.95901700 & -2.06814700 \\
\hline $\mathrm{H}$ & 0.28624800 & -2.57621000 & -2.15084500 \\
\hline $\mathrm{H}$ & -0.06056500 & -0.82808300 & -2.46260000 \\
\hline $\mathrm{C}$ & 0.97349500 & 1.97566900 & 3.69881800 \\
\hline $\mathrm{H}$ & 0.98746000 & 3.00069800 & 4.09857400 \\
\hline $\mathrm{H}$ & 0.74327800 & 1.30397100 & 4.53788600 \\
\hline $\mathrm{H}$ & 0.15877800 & 1.89993600 & 2.96384600 \\
\hline $\mathrm{C}$ & 4.68449100 & 2.05892000 & -1.40122500 \\
\hline $\mathrm{H}$ & 5.17539400 & 1.14428200 & -1.03344700 \\
\hline $\mathrm{C}$ & 4.72022200 & -0.19412500 & 1.22651300 \\
\hline $\mathrm{H}$ & 4.64471800 & -0.24997700 & 2.32621900 \\
\hline $\mathrm{H}$ & 5.64070000 & 0.34391100 & 0.96416900 \\
\hline $\mathrm{C}$ & -3.95539100 & -0.65144300 & 3.94119100 \\
\hline $\mathrm{H}$ & -4.94911400 & -0.63790500 & 3.46970500 \\
\hline $\mathrm{H}$ & -3.84624000 & 0.26251800 & 4.54333600 \\
\hline $\mathrm{H}$ & -3.92431600 & -1.51078800 & 4.62747700 \\
\hline $\mathrm{C}$ & -3.81756700 & 4.51362000 & 1.94533000 \\
\hline $\mathrm{H}$ & -3.77310300 & 5.58981000 & 1.71867100 \\
\hline $\mathrm{H}$ & -3.93202900 & 4.40145300 & 3.03327900 \\
\hline $\mathrm{H}$ & -4.71721300 & 4.11107900 & 1.45845300 \\
\hline $\mathrm{C}$ & -1.30731300 & 4.38521400 & 2.15615900 \\
\hline $\mathrm{H}$ & -0.38161400 & 3.93165600 & 1.76800400 \\
\hline $\mathrm{H}$ & -1.35754900 & 700 & 1200 \\
\hline $\mathrm{H}$ & -1.23728700 & 5.47461700 & 2.01953700 \\
\hline $\mathrm{C}$ & 0.40929000 & -4.09059700 & 2.54093900 \\
\hline $\mathrm{H}$ & -0.43648500 & -3.76755800 & 1.91932600 \\
\hline $\mathrm{H}$ & 0.23533700 & -3.74662800 & 3.57133100 \\
\hline $\mathrm{H}$ & 0.43180900 & -5.19084200 & 2.56740700 \\
\hline $\mathrm{C}$ & -5.43270900 & -3.06599600 & -1.78844900 \\
\hline $\mathrm{H}$ & -5.74435300 & -4.07426400 & -1.47646600 \\
\hline $\mathrm{H}$ & -5.73230100 & -2.92743200 & -2.83774800 \\
\hline $\mathrm{H}$ & -5.98680900 & -2.34636600 & -1.17075900 \\
\hline $\mathrm{C}$ & -3.20263900 & -3.95667600 & -2.50664000 \\
\hline $\mathrm{H}$ & -2.11986600 & -3.98360600 & -2.32164100 \\
\hline $\mathrm{H}$ & -3.36938400 & -3.73567700 & -3.57064800 \\
\hline $\mathrm{H}$ & -3.60031600 & -4.96522000 & -2.32154600 \\
\hline $\mathrm{C}$ & 4.98976500 & -2.71359000 & -2.94606800 \\
\hline $\mathrm{H}$ & 5.58454100 & -3.04537100 & -2.08263100 \\
\hline $\mathrm{H}$ & 5.58190500 & -1.98368100 & -3.51753100 \\
\hline $\mathrm{H}$ & 4.82731300 & -3.59145100 & -3.58953900 \\
\hline $\mathrm{C}$ & 3.41934600 & 1.67131000 & 4.16182500 \\
\hline $\mathrm{H}$ & 4.40917300 & 1.41684300 & 3.75752900 \\
\hline $\mathrm{H}$ & 3.19731800 & 0.99035800 & 4.99624100 \\
\hline $\mathrm{H}$ & 3.48280200 & 2.69408800 & 4.56332900 \\
\hline
\end{tabular}

$\begin{array}{lrrr}\mathrm{C} & -4.23434600 & 1.58249700 & -3.56034400 \\ \mathrm{H} & -4.90084000 & 1.60312300 & -2.68683600 \\ \mathrm{H} & -4.45455700 & 0.67333400 & -4.13930500 \\ \mathrm{H} & -4.48085200 & 2.45435100 & -4.18489300 \\ \mathrm{C} & -1.86149100 & 1.55222500 & -4.38847800 \\ \mathrm{H} & -2.10763100 & 2.33953700 & -5.11584200 \\ \mathrm{H} & -2.00135200 & 0.58746300 & -4.89625800 \\ \mathrm{H} & -0.79711800 & 1.64907900 & -4.12827100 \\ \mathrm{C} & 2.84055100 & -1.67359700 & -3.74578300 \\ \mathrm{H} & 2.55982700 & -2.53717600 & -4.36694000 \\ \mathrm{H} & 3.43738500 & -0.99637500 & -4.37389100 \\ \mathrm{H} & 1.92076800 & -1.14970600 & -3.45310700 \\ \mathrm{C} & 2.90837100 & -4.05459000 & 2.81590000 \\ \mathrm{H} & 2.99529400 & -5.14327400 & 2.68136400 \\ \mathrm{H} & 2.77538600 & -3.85278800 & 3.88871900 \\ \mathrm{H} & 3.85827400 & -3.60307500 & 2.49926300 \\ \mathrm{C} & 3.68087900 & 1.62807900 & -2.47857600 \\ \mathrm{H} & 2.89903100 & 0.96876600 & -2.07041000 \\ \mathrm{H} & 4.19692100 & 1.09030800 & -3.28799800 \\ \mathrm{H} & 3.18910100 & 2.50996700 & -2.91836700 \\ \mathrm{C} & 5.76646700 & 2.95279400 & -2.00479200 \\ \mathrm{H} & 5.33032300 & 3.81937800 & -2.52345500 \\ \mathrm{H} & 6.34446900 & 2.38864700 & -2.74995200 \\ & 6.46219100 & 3.32485800 & -1.23952800\end{array}$

\section{trans-3a' ${ }^{-+}$with M06-2X}

$\begin{array}{llll}\mathrm{P} & -0.15882600 & -1.29761700 & -0.06255600\end{array}$

$\begin{array}{llll}\mathrm{P} & 0.20709100 & 1.33078300 & 0.04783100\end{array}$

$\begin{array}{llll}\mathrm{N} & 3.22362500 & -1.53022700 & -0.24570100\end{array}$

$\begin{array}{llll}\mathrm{N} & -3.14057300 & 1.63892700 & 0.18243000\end{array}$

$\begin{array}{llll}\mathrm{N} & 3.53165400 & 0.61662900 & 0.24241300\end{array}$

$\begin{array}{llll}\mathrm{N} & -3.51488900 & -0.52983400 & 0.42608600\end{array}$

$\begin{array}{llll}\text { C } & -2.54964800 & 0.40626300 & 0.19252200\end{array}$

$\begin{array}{llll}\text { C } & 2.58785100 & -0.34198800 & -0.00750300\end{array}$

$\begin{array}{llll}\text { C } & 3.30749800 & 1.85975800 & 0.91349400\end{array}$

$\begin{array}{llll}\text { C } & 2.50574300 & -3.87641900 & -0.18733700\end{array}$

$\begin{array}{llll}\text { C } & 2.63677500 & -2.66149800 & -0.89435200\end{array}$

$\begin{array}{llll}\text { C } & 2.86493100 & 1.85165400 & 2.25377200\end{array}$

$\begin{array}{llll}\text { C } & -3.31916100 & -1.93232300 & 0.61051000\end{array}$

$\begin{array}{llll}\text { C } & 1.21761500 & -0.14595200 & -0.01159000\end{array}$

$\begin{array}{llll}\text { C } & 4.66322000 & -1.41488500 & -0.00505000\end{array}$

$\begin{array}{llll}\mathrm{H} & 5.22386000 & -1.92205700 & -0.80160500\end{array}$

$\begin{array}{llll}\mathrm{H} & 4.93652600 & -1.87106500 & 0.96060400\end{array}$

$\begin{array}{llll}\text { C } & 3.59007800 & 3.06689100 & 0.23858000\end{array}$ 


\begin{tabular}{|c|c|c|c|c|c|c|c|}
\hline $\mathrm{C}$ & 2.22194900 & -2.54226700 & -2.23713900 & $\mathrm{H}$ & -3.80660100 & 0.92750400 & -1.99743200 \\
\hline $\mathrm{C}$ & -3.47825500 & -2.47301200 & 1.90278600 & $\mathrm{C}$ & -1.85489200 & 5.05236000 & -1.89960800 \\
\hline $\mathrm{C}$ & -1.18989000 & 0.18516400 & 0.00946400 & $\mathrm{H}$ & -1.54584900 & 5.94807300 & -2.44067200 \\
\hline $\mathrm{C}$ & 4.88037800 & 0.09831200 & 0.00074100 & $\mathrm{C}$ & -0.11332900 & -2.17882000 & 1.55902000 \\
\hline $\mathrm{H}$ & 5.56228500 & 0.42825700 & 0.79560600 & $\mathrm{H}$ & -0.81483800 & -3.02294800 & 1.56746300 \\
\hline $\mathrm{H}$ & 5.26962400 & 0.45864400 & -0.96559200 & $\mathrm{H}$ & -0.33247700 & -1.47888900 & 2.37420900 \\
\hline $\mathrm{C}$ & -2.65434700 & 2.77962900 & -0.52449700 & $\mathrm{H}$ & 0.91248200 & -2.55405700 & 1.67296600 \\
\hline $\mathrm{C}$ & 3.07986700 & -4.05926300 & 1.20890500 & $\mathrm{C}$ & 1.45159300 & -4.84379200 & -2.14901800 \\
\hline $\mathrm{H}$ & 3.14631900 & -3.06140200 & 1.67541900 & $\mathrm{H}$ & 0.97883100 & -5.69649300 & -2.63881000 \\
\hline $\mathrm{C}$ & -2.81131600 & 2.82089000 & -1.92571000 & $\mathrm{C}$ & -2.82043600 & -4.10993200 & -0.28564300 \\
\hline $\mathrm{C}$ & 2.62850200 & 0.57513700 & 3.04686500 & $\mathrm{H}$ & -2.56098200 & -4.75676800 & -1.12398900 \\
\hline $\mathrm{H}$ & 2.69366600 & -0.28497200 & 2.36650200 & $\mathrm{C}$ & 1.23243600 & 0.54847700 & 3.67657400 \\
\hline $\mathrm{C}$ & -2.11244800 & 3.85853400 & 0.19907000 & $\mathrm{H}$ & 1.12265100 & 1.33074200 & 4.44250800 \\
\hline $\mathrm{C}$ & -3.01551300 & -2.74370300 & -0.50588500 & $\mathrm{H}$ & 1.05500700 & -0.41943600 & 4.16925900 \\
\hline $\mathrm{C}$ & 2.68867400 & 3.08045300 & 2.89485100 & $\mathrm{H}$ & 0.45208200 & 0.70737600 & 2.91621300 \\
\hline $\mathrm{H}$ & 2.35014500 & 3.09744800 & 3.93264600 & $\mathrm{C}$ & 0.51227600 & 2.20147600 & -1.54972300 \\
\hline $\mathrm{C}$ & -2.01293200 & 3.82360100 & 1.71373100 & $\mathrm{H}$ & 1.60194200 & 2.33426800 & -1.60059600 \\
\hline $\mathrm{H}$ & -2.11177700 & 2.77061000 & 2.02153900 & $\mathrm{H}$ & 0.02527100 & 3.18479400 & -1.55969000 \\
\hline $\mathrm{C}$ & 1.89722300 & -4.95463200 & -0.83607600 & $\mathrm{H}$ & 0.18160100 & 1.59728500 & -2.40298700 \\
\hline $\mathrm{H}$ & 1.77731600 & -5.90107300 & -0.30757000 & $\mathrm{C}$ & -2.82912800 & -1.78173900 & 4.25373100 \\
\hline $\mathrm{C}$ & -3.83672400 & -1.62139000 & 3.11093500 & $\mathrm{H}$ & -2.80456000 & -2.81754500 & 4.62302600 \\
\hline $\mathrm{H}$ & -3.81011500 & -0.56507900 & 2.80325900 & $\mathrm{H}$ & -3.10995500 & -1.13622200 & 5.09821800 \\
\hline $\mathrm{C}$ & -4.53556000 & 1.55977200 & 0.61318100 & $\mathrm{H}$ & -1.81286400 & -1.50912400 & 3.93917200 \\
\hline $\mathrm{H}$ & -5.16537200 & 2.19783700 & -0.02140200 & $\mathrm{C}$ & -2.98605800 & -2.17602000 & -1.91718400 \\
\hline $\mathrm{H}$ & -4.63798700 & 1.89620300 & 1.65876100 & $\mathrm{H}$ & -2.64205900 & -1.13432800 & -1.85281800 \\
\hline $\mathrm{C}$ & -1.71479600 & 4.99349700 & -0.51560900 & $\mathrm{C}$ & -4.85005100 & 0.07104500 & 0.45899200 \\
\hline $\mathrm{H}$ & -1.29664100 & 5.84697600 & 0.02068200 & $\mathrm{H}$ & -5.43265000 & -0.32926900 & 1.29822700 \\
\hline $\mathrm{C}$ & 2.42200100 & -1.28042800 & -3.06179000 & $\mathrm{H}$ & -5.39699200 & -0.14595700 & -0.47287100 \\
\hline $\mathrm{H}$ & 2.80074700 & -0.48390700 & -2.40621800 & $\mathrm{C}$ & 3.71928300 & 0.40215100 & 4.11097900 \\
\hline $\mathrm{C}$ & -3.31810200 & -3.85359800 & 2.06139100 & $\mathrm{H}$ & 4.72145400 & 0.39019000 & 3.65776100 \\
\hline $\mathrm{H}$ & -3.44755400 & -4.29872900 & 3.04990500 & $\mathrm{H}$ & 3.57663800 & -0.53944900 & 4.66126400 \\
\hline $\mathrm{C}$ & 1.62657500 & -3.65207700 & -2.84274200 & $\mathrm{H}$ & 3.69221700 & 1.22743500 & 4.83797400 \\
\hline $\mathrm{H}$ & 1.29463400 & -3.58053900 & -3.88058800 & $\mathrm{C}$ & 4.49674600 & -4.64790700 & 1.11271900 \\
\hline $\mathrm{C}$ & -2.39390600 & 3.97399600 & -2.59634700 & $\mathrm{H}$ & 4.44861600 & -5.66102300 & 0.68618100 \\
\hline $\mathrm{H}$ & -2.50325900 & 4.03420900 & -3.68120300 & $\mathrm{H}$ & 4.95787500 & -4.71972200 & 2.10870400 \\
\hline $\mathrm{C}$ & 2.94994600 & 4.28000000 & 2.24185200 & $\mathrm{H}$ & 5.15426300 & -4.05250400 & 0.46559400 \\
\hline $\mathrm{H}$ & 2.80710200 & 5.22814400 & 2.76247900 & $\mathrm{C}$ & 2.22656400 & -4.95313200 & 2.11459100 \\
\hline $\mathrm{C}$ & 3.39887200 & 4.26926100 & 0.92658100 & $\mathrm{H}$ & 1.16965500 & -4.65398600 & 2.12359400 \\
\hline $\mathrm{H}$ & 3.61354800 & 5.21361100 & 0.42456800 & $\mathrm{H}$ & 2.60690300 & -4.91183200 & 3.14506400 \\
\hline $\mathrm{C}$ & -2.97740900 & -4.66299700 & 0.98335500 & $\mathrm{H}$ & 2.27729000 & -6.00454700 & 1.79645000 \\
\hline $\mathrm{H}$ & -2.84014500 & -5.73545700 & 1.12958200 & $\mathrm{C}$ & -0.66288900 & 4.32984900 & 2.22333100 \\
\hline $\mathrm{C}$ & 4.16809400 & 3.09025000 & -1.16731600 & $\mathrm{H}$ & 0.16878600 & 3.79016600 & 1.74793700 \\
\hline $\mathrm{H}$ & 3.92488400 & 2.12509800 & -1.64328900 & $\mathrm{H}$ & -0.58984400 & 4.17965700 & 3.31092600 \\
\hline $\mathrm{C}$ & -3.42945700 & 1.67472300 & -2.71281200 & $\mathrm{H}$ & -0.53380500 & 5.40634300 & 2.03293100 \\
\hline
\end{tabular}




$\begin{array}{lrrrrrrr}\mathrm{C} & 5.69717300 & 3.23301400 & -1.10278200 & \mathrm{C} & 1.10109200 & -0.78990400 & -3.66112900 \\ \mathrm{H} & 5.96079300 & 4.21416600 & -0.67990700 & \mathrm{H} & 0.71966700 & -1.49356100 & -4.41671500 \\ \mathrm{H} & 6.13855400 & 3.16639200 & -2.10810700 & \mathrm{H} & 1.23845400 & 0.18364500 & -4.15569400 \\ \mathrm{H} & 6.16284200 & 2.46889400 & -0.46628500 & \mathrm{H} & 0.33272000 & -0.68523600 & -2.87981300 \\ \mathrm{C} & 3.59610500 & 4.20912200 & -2.04411600 & \mathrm{C} & -2.38428500 & 0.97324200 & -3.58556000 \\ \mathrm{H} & 2.49792300 & 4.22504900 & -2.03951700 & \mathrm{H} & -1.93452200 & 1.67611800 & -4.30427100 \\ \mathrm{H} & 3.93600000 & 4.08177400 & -3.08170600 & \mathrm{H} & -2.84756100 & 0.15405800 & -4.15630700 \\ \mathrm{H} & 3.94917700 & 5.19524700 & -1.70965100 & \mathrm{H} & -1.58108800 & 0.54609700 & -2.96773100 \\ \mathrm{C} & -4.62269500 & 2.14547200 & -3.55007500 & \mathrm{C} & -3.16696100 & 4.61972300 & 2.33632800 \\ \mathrm{H} & -5.37044400 & 2.66090200 & -2.93031400 & \mathrm{H} & -3.09198600 & 5.68284400 & 2.06101700 \\ \mathrm{H} & -5.10842200 & 1.28728000 & -4.03692700 & \mathrm{H} & -3.13883700 & 4.55066800 & 3.43351500 \\ \mathrm{H} & -4.30696100 & 2.84023000 & -4.34204000 & \mathrm{H} & -4.14637000 & 4.25804300 & 1.99080800 \\ \mathrm{C} & -5.25191000 & -1.95161100 & 3.60307200 & \mathrm{C} & -2.02633700 & -2.91087300 & -2.85191900 \\ \mathrm{H} & -6.00037600 & -1.86174800 & 2.80237100 & \mathrm{H} & -1.02489500 & -3.00265500 & -2.40671900 \\ \mathrm{H} & -5.54141000 & -1.28345600 & 4.42695700 & \mathrm{H} & -1.93515400 & -2.35618300 & -3.79790900 \\ \mathrm{H} & -5.29833600 & -2.98518500 & 3.97798400 & \mathrm{H} & -2.39392800 & -3.91785400 & -3.10214700 \\ \mathrm{C} & 3.47301000 & -1.51541000 & -4.15306400 & \mathrm{C} & -4.40226800 & -2.17181700 & -2.50846400 \\ \mathrm{H} & 4.43081400 & -1.84181100 & -3.72236700 & \mathrm{H} & -4.78343600 & -3.20024300 & -2.59858900 \\ \mathrm{H} & 3.64686600 & -0.59348400 & -4.72695200 & \mathrm{H} & -4.40037200 & -1.72092500 & -3.51241800 \\ \mathrm{H} & 3.14084500 & -2.29386500 & -4.85603700 & \mathrm{H} & -5.10932400 & -1.61158400 & -1.88140600\end{array}$

\section{S5: References}

[1] a) Z. Li, X. Chen, D. M. Andrada, G. Frenking, * Z. Benkö, Y. Li, J. R. Harmer, C.-Y. Su,* H. Grützmacher,* Angew. Chem. Int. Ed. 2017, 56, 5744-5749; Angew. Chem. 2017, 129, 5838-5843;

[2] M. J. Frisch, G. W. Trucks, H. B. Schlegel, G. E. Scuseria, M. A. Robb, J. R. Cheeseman, G. Scalmani, V. Barone, B. Mennucci, G. A. Petersson, H. Nakatsuji, M. Caricato, X. Li, H. P. Hratchian, A. F. Izmaylov, J. Bloino, G. Zheng, J. L. Sonnenberg, M. Hada, M. Ehara, K. Toyota, R. Fukuda, J. Hasegawa, M. Ishida, T. Nakajima, Y. Honda, O. Kitao, H. Nakai, T. Vreven, J. J. A. Montgomery, J. E. Peralta, F. Ogliaro, M. Bearpark, J. J. Heyd, E. Brothers, K. N. Kudin, V. N. Staroverov, R. Kobayashi, J. Normand, K. Raghavachari, A. Rendell, J. C. Burant, S. S. Iyengar, J. Tomasi, M. Cossi, N. Rega, J. M. Millam, M. Klene, J. E. Knox, J. B. Cross, V. Bakken, C. Adamo, J. Jaramillo, R. Gomperts, R. E. Stratmann, O. Yazyev, A. J. Austin, R. Cammi, 
C. Pomelli, J. W. Ochterski, R. L. Martin, K. Morokuma, V. G. Zakrzewski, G. A. Voth, P. Salvador, J. J. Dannenberg, S. Dapprich, A. D. Daniels, O. Farkas, J. B. Foresman, J. V. Ortiz, J. Cioslowski, D. J. Fox, Gaussian 09, Revision C.01. Gaussian, Inc., Wallingford CT, 2009.

[3] Perdew, J.P., Ziesche, P. and Eschrig, H., 1991. Electronic structure of solids' 91.

[4] [a] Grimme, S.; Antony, J.; Ehrlich, S.; Krieg, H. J. Chem. Phys. 2010, 132, 154104. [b] Grimme, S. J. Comput. Chem. 2006, 27, 1787. [c] Grimme, S. J. Comput. Chem. 2004, 25, 1463.

[5] A. Bondi, J. phys. Chem. 1964, 68.

[6] Perdew, J. P.; Burke, K.; Ernzerhof, M. Phys. Rev. Lett. 1997, 78, 1396.

[7] Becke, A. D. J. Chem. Phy. 1993, 98, 5648. 